\title{
Chapter 2: Physical, chemical, and petrological characteristics of chondritic materials and their relationships to small Solar System bodies
}

Michael E. Zolensky

KT Astromaterials Research and Exploration Science

NASA Johnson Space Center

Houston, TX 77058, USA

Neyda M. Abreu

The Pennsylvania State University - DuBois Campus

1 College Place

DuBois, PA 15801, USA

Michael A. Velbel

Department of Earth and Environmental Sciences

288 Farm Lane, room 207 Natural Science Building

Michigan State University

East Lansing, MI 48824-1115, USA

and

Division of Meteorites

Department of Mineral Sciences

National Museum of Natural History

Smithsonian Institution

Washington, DC 20013-7012, USA

Alan Rubin

Earth, Planetary, and Space Sciences

Institute of Geophysics and Planetary Physics

University of California - Los Angeles

Los Angeles, CA 90095-1567, USA

Noel Chaumard

University of Wisconsin-Madison | UW

Department of Geoscience

Madison, Wisconsin, USA

Takaaki Noguchi

Faculty of Arts and Science, Kyushu University

744, Motooka, Nishi-ku, Fukuoka 819-0395, Japan

Tatsuhiro Michikami

Faculty of Engineering

Kinki University

1 Takaya Umenobe, Higashi-Hiroshima

Hiroshima 739-2116, Japan 


\begin{abstract}
Chondrite materials with varying abundances of volatile-bearing phases are expected at the destinations for the asteroid sample-return missions Hayabusa2 and OSIRIS-REx. The targets of the missions are 162173 (1999 JU3) Ryugu and 101955 (1999 RQ36) Bennu. Spectroscopic analyses of these asteroids suggest that their surface materials are related to types 1 and 2 carbonaceous chondrites. Some studies suggest that the parent bodies of these chondrites may have also experienced some thermal and/or shock metamorphism. The physical properties of boulders at asteroid surfaces and fine particles in asteroid regoliths are consequences of the diverse processes that fragmented them, mobilized them, and redeposited them in unique accumulations. Sample-return missions are likely to encounter a broad range of carbonaceous chondrite (CC)-like materials, to which aqueous alteration, thermal, and shock metamorphism imparted changes affecting their sub-micron- to meter-scale physical properties. Consequently, implementation of scale-dependent analytical techniques to the study of the chemical, physical, and geotechnical characteristics of these CC-like materials is fundamental to safe mission operations, sample selection, and return. However, most of the available knowledge for informing and formulating expectations about regolith processes, products, and properties on carbonaceous small bodies comes from missions that studied anhydrous (e.g., Itokawa studied by Hayabusa) and/or much larger asteroids (e.g., Vesta studied by Dawn). No previous mission is likely directly relevant to small ice-free carbonaceous NEOs 162173 Ryugu or 101955 Bennu, although the Rosetta Spaceraft performed a flyby of the large asteroid Lutetia which has variously been classified as $\mathrm{M}$ and $\mathrm{C}$ type (Pätzold et al., 2011). Carbonaceous chondrites carry the best record of the history, distribution, and activity of water in the early solar system. Ordinary and Enstatite chondrites carry only partial records, but these are still critical to understanding the full story. We will describe the records of water-rock interactions on asteroids, as recorded in these meteorites, with particular emphasis on the timing, nature, settings, and fluid compositions. An integral part of this story is the rare, but fortunate, preservation of actual early solar system water as aqueous fluid inclusions.
\end{abstract}

\title{
2.1. Introduction
}

Our main source of extraterrestrial materials is meteorites, or the rocks that "rained" on earth, which are either observed as they fall or recognized and collected. The number of meteorites available for research has grown exponentially since American, Japanese, and other teams began organizing expeditions to retrieve these rocks from cold and hot deserts, accounting for nearly all the mass available across tens of thousands of individual samples. In addition to these rocks, extraterrestrial dust is collected via high altitude flights. Even though we are now entering the era of interplanetary sample return missions, aside from lunar samples collected in the 1960's, spacecraft have only retrieved less than one gram of extraterrestrial mass from one asteroid and one comet.

Chondrites are aggregates of materials with significantly variable histories, mineralogies, grain sizes and shapes, porosities, and physical strengths. Throughout their histories components and current lithologies of chondrites have experienced condensation from gas or liquids, heating and partial to complete vaporization, radiation damage and implantation of solar wind gas, atoms, nuclei and electrons, accretionary and later stage shock metamorphism, dynamic and thermal metamorphism, sulfidation, and aqueous alteration (Hutchison, 2004). This record is 
preserved in the chemical and istopic characteristics of these samples. A myriad of stable and radiogenic isotopes have been measured for chondrites. For the purpose of this chapter, we focus on the oxygen and Al-Mg systematics of chondrites. This selection was based on understanding the history of water in chondrites and not the scientific importance of other systems, which are critical to our understanding of early solar system history.

Chondrites carry the best record of the history, distribution, and activity of water in the early solar system. Chondritic meteorites, especially carbonaceous chondrites, have special significance cosmochemically. They are similar in composition to the solar photosphere, excluding a few very volatile elements (e.g., Sears and Dodd, 1988). They are samples of primitive, in other words, undifferentiated materials that are found as asteroids in the belt between Mars and Jupiter, as near earth objects (NEOs), and potentially as cometary materials.

Chondrites consist of ferromagnesian igneous spherules called chondrules and refractoryelement rich inclusions (i.e., Calcium Aluminum-rich Inclusions or CAIs and Amoeboid Olivine Aggregates or AOAs) embedded in a sub-micron silicate-rich matrix. It is widely accepted that processes in the solar nebula resulted in the formation of the high-temperature components, chondrules and refractory inclusions. After their formation, chondrules and CAIs accreted along with surviving interstellar grains, fine-grained matrices, and carbonaceous materials into asteroidal parent bodies.

Chondrules are thought to have formed within the first few million years of solar system history. Although for a long time it was thought that chondrules formed relatively late in the evolution of the solar nebula, a more recent $\mathrm{U}$-corrected $\mathrm{Pb}-\mathrm{Pb}$ study indicates that at least some chondrules have formation ages ranging from $4567.32 \pm 0.42$ to $4564.71 \pm 0.30 \mathrm{Myr}$, which overlaps with the time in which CAIs formed (Connelly et al., 2012). Mineralogically, chondrules are dominated by olivine, pyroxenes, a feldspathic mesostasis, and Fe-rich opaque minerals (e.g., Rubin 2010; Jones 2012). In terms of textures, chondrules are predominantly porphyritic (over $80 \%$ - Jones 2012), including porphyritic olivine and pyroxene (POP), porphyritic olivine (PO), and porphyritic pyroxene (PP) varieties. Non-porphytic textures include barred olivine (BO), radiating pyroxene (RP), and cryptocrystalline (CC) textural types (e.g., Rubin, 2010; Jones, 2012). Chondrules are classified based on their FeO contents, into type I chondrules (FeO-poor; $\mathrm{Fe} /(\mathrm{Fe}+\mathrm{Mg}$ ) ratios <10 mole\%.), type II chondrules (FeO-rich; Fe/(Fe + $\mathrm{Mg}$ ) ratios $>50$ mole $\%$ ) and comprise as little as $1 \mathrm{vol} \%$ in some chondrite groups, and Aluminum-rich chondrules (ARCs) (e.g., Weisberg et al., 1993; Krot and Keil, 2002; Connolly et al., 2007; Connolly and Huss 2010). In a $\delta^{17} \mathrm{O}$ vs. $\delta^{18} \mathrm{O}$ plot, chondrules from primitive chondrites plot on a line with slope $\sim 1$ and type I chondrules are often enriched in ${ }^{16} \mathrm{O}$ with respect to type II chondrules (e.g., Clayton, 1993; Krot et al., 2006a, 2010; Connolly and Huss, 2010; Libourel and Chaussidon, 2011; Ushikubo et al. 2012; Schrader et al., 2013, 2014; Tenner et al., 2013, 2015). Numerous experimental and petrologic studies indicate that chondrules formed by rapid (seconds to minutes), partial to complete melting of dust precursors (e.g., Hewins et al., 2005; Connolly and Jones, 2016 and references therein). However, the mechanism of chondrule formation has long been a subject of debate. Shocks, magnetized turbulence, and impacts have been considered as potential drivers for chondrule formation (see Krot and Nagashima, 2017 and references therein). In addition to condensation, chondrules record a variety of other processes occurring in the nebula, asteroids, and on earth (e.g., multiple condensation/heating events, collisions with other chondrules, elemental exchange with nebular gas, metamorphism, aqueous alteration). Therefore, the characteristics of chondrules can be used to explore a variety of environments. For example, isotopic compositions of chondrules have 
been used to explore the characteristics of nebular gas and dust, as well as the processes operating in different locations of the solar nebula. Authors have argued that the oxygen isotopic compositions of chondrules in different chondrite groups can be attributed to formation at different heliocentric distances (e.g., Krot et al. 2006) and at different times (e.g., Amelin et al., 2002).

Refractory-element rich inclusions represent up to 10 vol.\% of chondrites and include to very different types of aggregates, CAIs and AOAs. CAIs are thought to be the first solids condensed from the solar nebula, 4567.2 $\pm 0.6 \mathrm{Ma}$ (canonical value) based on their $\mathrm{Pb}-\mathrm{Pb}$ ages and a host of isotopic anomalies, including the highest ${ }^{26} \mathrm{Al} /{ }^{27} \mathrm{Al}$ ratio, the lowest ${ }^{87} \mathrm{Sr} /{ }^{86} \mathrm{Sr}$ ratio, and a record of the short-lived radionuclides ${ }^{41} \mathrm{Ca}$ and ${ }^{10} \mathrm{Be}$ (e.g., Lee et al., 1976; McKeegan and Davis, 2003; McPherson et al. 1988, 1995, 2005, 2010; MacPherson, 2014). In addition, CAIs have the lowest $\delta^{17} \mathrm{O}$ and $\delta^{18} \mathrm{O}$, plotting near solar compositions (e.g., Clayton, 1993; Gounelle et al., 2009; McKeegan et al., 2011). Texturally, CAIs are made up of interior crystals overlaid by a sequence of monominerallic layers termed Wark-Lovering rims. Mineralogically, CAIs are very diverse. CAIs care predominantly made up of spinel, melilite, perovskite, hibonite, calcic pyroxenes, anothite, and forsterite, which dominate both the interior and the Wark-Lovering rims. Other minerals present in CAIs are grossite, corundum, Fe-Ni metal, Fe-rich opaque assemblages, and PGE nodules (e.g., MacPherson 2014 and references therein). As chondrules, CAIs experienced reheating events and elemental exchange with the nebular gas as well as a varierty of processes in their asteroidal parent bodies, and in some cases in the terrestrial environment. AOAs are irregularly shaped, fine-grained, forsterite $(\mathrm{Fa}<2)$-rich inclusions, which may also contain kamacite (5-12 wt\% Ni), and CAI fragments (e.g., Grossman and Steele, 1976; Cohen et al., 1983; Rubin, 1985; Chizmadia et al., 2002; Krot et al., 2004a; Weisberg et al., 2004). Consequently, AOAs are not as refractory as CAIs. CAIs in AOAs are composed of Aldiopside, spinel, anorthite, and scarce melilite (e.g., Krot et al. 2004a; Weisberg et al. 2004). Forsterite and spinel in AOAs are ${ }^{16}$ O-rich (e.g., Krot et al. 2004a). Based on their concetrations of minor elements, Sugiura et al (2009) argued that AOAs formed either by slow reheating of porous aggregates or by condensation and aggregation of forsterite in a slow cooling nebular region. As in the case of chondrules and CAIs, AOAs may have experienced post-condensation heating and isotopic exchange with nebular gases (e.g., Krot et al., 2004a; Weisberg et al., 2004).

Matrices are complex mixtures of micron and sub-micron silicates, sulfides, oxides, and organics, and a host of accessory minerals, whose origins are the subject of considerable debate. A small component of matrices certainly consists of interstellar and circumstellar materials, including silicates (Nguyen and Zinner 2004) and organic materials. However, the bulk of the matrix probably represents interstellar dust that was processed in a variety of ways in the solar nebula and hence can be considered to be of local origin (e.g. Nuth et al. 2005).

Considering how varied and numerous asteroids are, it is not surprising that there is also a great deal of variation in types of chondrites: ordinary, carbonaceous, enstatite, R-type, as well as several minor groups. Classification schemes of the chondrites have been extensively discussed in the literature (e.g., Wasson 1985; Kallemeyn et al. 1996; Krot et al. 2004; Weisberg et al. 2006). In this chapter, we follow the guidelines established by Weisberg et al. (2006) and Krot et al. (2014), in which the hierarchical classification of chondrites follow the order class, group, and subgroup categorization. Because the emphasis of this volume is on asteroidal exploration, this chapter focuses on ordinary and carbonaceous chondrites. These meteorites likely originated from asteroids that are current/future mission targets and are ideal candidates for in-situ resource 
utilization (ISRU). In particular, the characteristics of carbonaceous chondrites are presented in detail because contain the most carbon and are spectral matches for $\mathrm{C}$ and $\mathrm{D}$-type asteroids, which are the target objects for upcoming sample-return missions: Hayabusa2 from JAXA and OSIRIS REx from NASA. Brief descriptions of the other groups are provided for completeness.

The ordinary, carbonaceous, and enstatite chondrites are divided into these three major classes according to their bulk chemistry, their refractory element abundances, their ratio of oxidized to reduced iron, oxygen isotopes, and petrographic characteristics (e.g., chondrule diameters and frequency distribution of different chondrule textural types). The most abundant chondritic meteorites are the ordinary chondrites (OCs), which account for more than $80 \%$ of all falls, it is important to understand their properties and formations of their asteroidal parent bodies. OCs may be over-represented in our collections owing to the fact that they are sturdy meteorites, thus more likely to survive atmospheric entry. Although the Enstatite chondrites (ECs) are sturdy meteorites, they are very scarce. Carbonaceous chondrites (CCs) are much less common than ordinary chondrites because they may be preferentially destroyed during atmospheric entry owing to their lower strength, so their true relative abundance is unknown.

Ordinary chondrites are naturally delivered samples from asteroidal parent bodies in the inner Main Belt region of the asteroid belt, by way of Earth-crossing asteroids originally from the inner Main Belt (Grady and Wright, 2006; Nakamura et al., 2011). Ordinary chondrites differ from carbonaceous chondrites mainly in their lower abundances of volatile elements; ordinary chondrites are essentially anhydrous (with few exceptions) but otherwise primitive (e.g., Dodd, 1981; McSween, 1999; Krot et al., 2003; 2007; Hutchison, 2004; Weisberg et al., 2006). Three main groups of ordinary chondrites $-\mathrm{H}, \mathrm{L}$, and LL - differ mainly in the total abundance and oxidation state of $\mathrm{Fe}$, with associated variations in the abundances and compositions of the metal, sulfide and silicate minerals that host $\mathrm{Fe}^{\circ}$ and $\mathrm{Fe}^{2+}$ (Dodd, 1981; McSween, 1999; Krot et al., 2003; Hutchison, 2004; Weisberg et al., 2006).

Enstatite chondrites are among the most chemically reduced rocks known, with most of their iron as metal or in various sulfides, rather than being in silicates or oxides (Hutchison, 2004). Their most important constituent is enstatite, but there are also lesser amounts of high-Ca pyroxene, forsterite, plagioclase, Fe-Ni-Si metal, silica, troilite, and accessories - often quite strange, reduced phases. Enstatite chondrites are divided into two groups: EH (high enstatite) and EL (low enstatite) chondrites. EH chondrites contain small chondrules $(\sim 0.2 \mathrm{~mm})$ and high ratios of siderophile elements to silicon. EL (low enstatite) chondrites contain larger chondrules $(>0.5$ $\mathrm{mm}$ ), and low ratios of siderophile elements to silicon. Enstatite chondrite meteorites are devoid of hydrous phases, as would be expected from such reduced rocks, however the Kaidun breccia contains abundant clasts of hydrous EH and EL materials (Zolensky and Ivanov, 2003). It is not known whether this aqueous alteration occurred on the parent E-chondrite asteroid or on some more hydrous asteroid after transport of anhydrous precursors.

The Carbonaceous (C) chondrite class is characterized by close to solar elemental abundances, low iron-metal content, high carbon content and volatile element enrichments. As its name indicates, the carbonaceous group has the highest carbon concentration among chondritic meteorites (up to $5 \mathrm{wt} \%$; Table 2.1). They are also enriched in volatile elements (e.g., $\mathrm{H}_{2} \mathrm{O}>5 \%$; Table 2.1). However, their carbon and water contents vary widely, with some groups containing very little of each. Carbonaceous chondrites show a wide range in their textural characteristics, degree of oxidation, aqueous alteration, shock- and thermally-driven metamorphism, pointing to a complex nebular and asteroidal history. 
Carbonaceous chondrites are divided on the basis of chemical composition and oxygen isotope ratios into groups. As with other chondritic groups, each group of carbonaceous chondrites is designated by a letter (group). This letter generally signifies the type meteorite for that group, usually an observed fall. The CV (Vigarano-type), the CO (Ornans-type), the CM (Mighei-type), the CI (Ivuna-type), the CR (Renazzo-like), the CK (Karoonda-like), the CH (high-iron) and the CB Bencubbinite-like groups have been recognized.

The presence of non-volatile elements in CI meteorites similar in abundance to those in the solar photosphere suggested that these are the most primitive (least evolved) solids in the Solar System (e.g., Anders and Ebihara 1982; Anders and Grevasse 1989). At one time it was believed that such primitive and volatile-rich material (petrologic type 1) was a primary nebular condensate and therefore the "starting material" for subsequent parent body evolution, and that other groups of meteorites had been subjected to progressively greater degrees of devolatilization and phase equilibration. Vestiges of this idea survive as the petrologic-type ("metamorphic grade") designations in the Van Schmus and Wood (1967) classification of chondritic meteorites. Subsequent petrographic research recognized that at least some hydrous phyllosilicates in carbonaceous chondrites occur as aqueous alteration products of anhydrous precursors (e.g., DuFresne and Anders 1962; Bunch and Chang 1980). The dominant recent line of thought holds that petrologic type 3 was the "starting material"; in this view, types 4-6 represent prograde (thermal) metamorphism, and petrologic types 2 and 1 may be products of increasing degrees of hydrous alteration of type 3 starting material (McSween 1979a; Buseck and Hua 1993). CI chondrites are mostly petrologic type 1; CM chondrites are mostly petrologic type 2 (CM2; Dodd 1981; Brearley and Jones 1998; McSween 1999; Krot et al. 2003; Hutchison 2004; Weisberg et al. 2006).

As well as compositional differences, there are important petrologic differences among the carbonaceous chondrites groups. For example, chondrules are absent in CI chondrites, CVs and CRs contain large chondrules and COs are noted for their very small chondrules. CVs are also characterized by the highest abundance of $\mathrm{Ca}$, Al-rich inclusions (CAIs), that can reach over a centimeter in size (e.g., MacPherson et al., 1988; Krot et al. 2002). CAIs are refractory element-rich inclusions, composed mainly of oxides and silicates. Matrix abundance also varies between the different carbonaceous chondrite groups, being highest in the CIs and lowest in the $\mathrm{CV}$ s and COs. The CI, CR, and CM chondrites have been significantly affected by aqueous alteration, while COs and CVs are generally much less altered. CK chondrites contain homogeneous Fe-Mg silicates, abundant magnetite and the coarse-grained matrices and formed under very oxidizing conditions (Keller et al., 1993). They appear to be the only carbonaceous chondrite group that has experienced significant thermal metamorphism. The detailed characterisitcs of each of these carbonaceous chondrite groups and the potential locations where these characteristics where imparted are presented in subsequent section.

Chondritic materials were then subjected to a variety of additional processes such as aqueous alteration, thermal metamorphism and brecciation. The first step in realizing the scientific promise that chondrites hold is in understanding what processes occurred where and in what sequence. To understand this process, the changes that took place in asteroids are codified into the petrologic sequence (Van Schmus and Wood 1967). The effects of aqueous alteration and thermal metamorphism are encoded into a number.this sequence the least altered chondrites are designated petrologic type 3 . Increasing degrees of aqueous alteration are represented by the sequence 3 to 1 and increasing thermal metamorphism types, 3 to 6 . There is controversy as to where this alteration took place. It has been suggested that aqueous alteration of early 
condensates occurred as the solar nebula cooled (e.g., Metzler et al., 1992) or after accretion in an asteroidal environment (e.g., Brearley, 1997, 1999, Kojima and Tomeoka, 1993, 1996, Krot et al., 1995, 1997, 1998, Tomeoka and Buseck, 1990, Zolensky and McSween, 1988). The effects of these parent body alteration processes are difficult to separate from alteration that may have taken place prior to accretion.

Water, as a solvent and mass transport agent, is the main source of low-temperature chemical alteration in some chondrites. The array of oxidation, hydrolysis, and carbonation reactions resulting from aqueous fluids reacting with chondritic minerals are collectively termed aqueous alteration. The conditons for aqueous alteration in chondritic parent bodies varied significantly in terms of conditions of temperature (including temperatures below freezing), pressure, water to rock ratio, redox conditions, fluid mobilization, and escape of volatiles (e.g., Zolensky et al. 1993; Zolotov 2012). The study of aqueous alteration allows to place constraints on: (1) the volatile budget inherited from the solar nebula; (2) the extent of chemical redistribution, changes in petrologic relationships, and isotopic fractionation that took place in chondritic parent bodies; (3) the availability of water for in-situ resource utilization (ISRU) for future asteroidal missions. This understanding of aqueous alteration has come very gradually (e.g., Brearley, 2006), as many meteorites with no obvious record of aqueous alteration have ultimately come to be recognized as having been thoroughly altered, then thermally metamorphosed to largely erase the record (Tonui et al., 2014). In many instances the record of aqueous alteration is preserved only as rare fragments of lithologies, preserved in regolith breccias of otherwise anhydrous materials.

Chondritic parent bodies have been heated to a variety of temperatures, which operated in different timescales. The consequences of thermal metamorphism are textural, compositional, and mineralogical homogenization and loss of volatiles. Thermal metamorphism has been attributed to electromagnetic induction by solar winds, to decay of short-lived radionuclides such as ${ }^{26} \mathrm{Al}$ in the parent body, to heating during accretion or during later impact events (e.g., McSween et al., 1988). A preponderance of evidence indicates that most thermal metamorphic features were caused by isotopic decay and impact-driven thermal and hydrothermal metamorphism. The induced thermoluminescence (TF) properties, degree of homogeneity of matrix olivine, abundance of interstellar material, their noble gas content, as well as degree of organics crystallographic maturation have been used to determine the degree of thermal metamorphism affecting individual chondrites (Sears et al. 1980, 1991, 1995; Krot et al., 1995; Bonal et al. 2007, 2016). However, many of these thermal metamorphism indicators often conflict with each other.

There is not a single group of ordinary, enstatite, or carbonaceous chondrites in which all six petrologic types are represented. At best, there are large gaps in the degree to which members of a group have been aqueously or thermally altered (Figure 2.1). Such gaps suggest that our collections are incomplete and/or that there is a great deal of diversity in the nature and extent of secondary alteration affecting different parent bodies. Both suggestions are consistent with asteroidal observations. There are numerous ordinary chondrites with hydrous phases, but no examples of S type asteroids showing evidence of this, and D type asterods are very abundant, but few or no meteorites representative of this clast (a possible exception being Tagish Lake, Hiroi et al., 2001). For example, most ordinary and enstatite chondrites are nearly anhydrous or show evidence of limited aqueous alteration (especially LL3s, Krot et al., 1997). In contrast, many carbonaceous chondrites have been thoroughly aqueously altered (e.g., CI, CM1, and CR1 chondrites) (Hutchison, 2004). Hydrated chondritic groups vary widely in their contents of 
volatile species, especially structural water in "hydrous" (OH-bearing) minerals. Only one group of carbonaceous chondrites - the CK chondrites - show evidence of thermal metamorphism that equals or exceeds petrologic type 4 . In constrast, ordinary and enstatite chondrites span the thermal metamorphism scale.

Chondritic parent bodies are exposed to a variety of physical stressors including: accretionary processes, impacts, small to catastrophic collisional disruption, reaccretion, expansion and contraction driven by temperature and phase changes during aqueous alteration and thermal metamorphism, space weathering, solar heating, and insolation, which in particular for parent bodies in NEO orbits may lead to large changes in surficial temperatures, YORP, and Yarkovsky effects. The outcomes of physical stresses are collectively termed as brecciation, which has led to the formation of disaggregated regoliths and lithified breccias. Despite volumetric losses owing to ablation during atmospheric entry, chondrites that are larger than the breccia fragments themselves preserve a record of brecciation. Evidence of brecciation includes extensive fracturing, comminution, disaggregation, mobilization, and relithification that may have involved one or more parent bodies. These characterisitcs are generally attributed to accretionary processes, impact events, and regolith gardening (i.e., mixing). Studies of breccias have focused primarily on the ordinary chondrites (e.g., Bischoff et al. 2006, 2017), with a few exceptions, including carbonaceous chondrite and achondritic breccias (e.g., Bunch 1975; Kallemeyn et al. 1978; Ivanov et al. 1996; Zolensky et al. 1996; Zolensky and Ivanov 2003; MacPherson et al. 2009; Horstmann and Bischoff 2014; Harries and Zolensky 2016).

The physical properties of chondrites vary widely across ordinary, enstatite, or carbonaceous chondrites. Grains sizes vary down to submicron regime, although most chondrites contain abundant $100 \mu \mathrm{m}$ to $5 \mathrm{~cm}$-sized chondrules. Porosities vary from $\sim 50 \%$ down to $0 \%$ (Corrigan et al., 1997; Macke et al., 2011). Physical compressive strengths vary over 3 orders of magnitude, from 100-300 MPa for metamorphosed ordinary chondrites (Flynn, 2015) down to 0.7 MPa for the weakest chondrite, ungrouped carbonaceous chondrite Tagish Lake (Brown et al., 2002). Thermal conductivity and porosity of most chondrites are strongly correlated (Opeil et al., 2012), while the heat capacity of ordinary chondrites is similar to quartz (Consolmagno et al., 2013), which varies from 44 to $64 \mathrm{~J} / \mathrm{mol}^{*} \mathrm{~K}$ over the temperature range 298-600K. Presumably, the heat capacity is significantly different for porous chondrites and powdery asteroidal regolith, due to differences in degree of grain attachments surface areas.

In this chapter, we present the physical, chemical, and mineralogical characteristics of chondritic meteorites from an exploration perspective. Owing to this perspective, our emphasis is the characterization of ordinary chondrites and hydrated carbonaceous chondrites, namely the CM, CI, and CR chondrites and the mineral products of their aqueous alteration. Asteroids spectroscopically consistent with $\mathrm{CI}$ and CM chondrites were selected as targets of sample return missions, Hayabusa2 (JAXA) and OSIRIS REx (NASA). This selection was made on the basis of presumed high contents of hydrous $(\mathrm{OH}$-bearing) minerals and associated organics in each asteroid's spectroscopic-counterpart C- class. These or other carbonaceous chondritic materials may be encountered on the target asteroids of current and planned sample-return missions. Hydrated chondrites have the highest $\mathrm{H}_{2} \mathrm{O}$ and $\mathrm{C}$ abundances. A great diversity of what is known about hydrated chondites comes from CM chondrites because they are the most numerous (Table 2.1). The CR chondrites are included in this list because they were a spectral match to the target of the now cancelled ARRM mission. Discussions of characteristics that, while scientifically very important, have limited impact on exploration of chondritic parent bodies are 
beyond the scope of this chapter as are exclusively scientific implications of characteristics of chondritic meteorites.

Spectroscopic analyses of small-body mission targets suggest that surface materials are related to $\mathrm{C} 1$ and $\mathrm{C} 2$ chondrites, the most aqueously altered meteorites in terrestrial laboratories (Li et al., 2006; Hasegawa et al., 2008; Milliken and Rivkin, 2009; Campins et al., 2009; Rivkin and Volquardsen, 2010; Campins et al., 2010; Rivkin et al., 2011; Delbo and Michel, 2011; Committee on the Planetary Science Decadal Survey, 2011). All spectroscopic matches between mission-target asteroids and meteorites are with members of carbonaceous chondrite groups that contain hydrogen-bearing phyllosilicates and associated elevated abundances of carbonaceous materials (Dodd 1981; McSween 1999; Krot et al. 2003; Hutchison 2004; Weisberg et al. 2006; Velbel and Palmer 2011; Table 1). The presence of brucite, magnesite, and dolomite inferred from spectra of 1 Ceres suggest that 1 Ceres' surface materials are possibly more extensively modified by aqueous alteration than C1 chondrites (Li et al., 2006; Milliken and Rivkin, 2009; Rivkin and Volquardsen, 2010; Rivkin et al., 2011; Clark et al. this volume). However, without a sample return, this inference must remain tentative. The best spectroscopic matches to the target asteroid of the upcoming Hayabusa2 sample-return mission, 1621731999 JU3 Ryugu, are chondritic ("primitive"; Binzel et al., 2004), ALH 82001 (CM2) (Vilas et al., 2008), and Murchison (CM2) (Sugita et al., 2013). At the group level, CI and / or CM chondrites are the best spectroscopic matches to the target asteroid of the OSIRIS-REx sample-return mission (101955 1999 RQ 36 Bennu). Clark et al. (2011) suggest "CM1"-like material as a match for 101955 Bennu. The best spectroscopic matches of individual meteorites to Bennu are a thermally altered CI material, an anomalous CM chondrite, and a matrix-rich fraction of a normal CM chondrite (Clark et al., 2011). Hoiwever, we can probably expect to be surprised.

\subsection{Ordinary chondrites and their counterpart asteroids.}

\subsubsection{Physical properties of ordinary chondritic material}

Of the chondritic meteorites, the physical properties are best known for the ordinary chondrites. A recent review by Flynn et al. (2017) summarizes the physical properties of this and other chondritic groups, and the majority of the information below is abstracted from this useful reference. The average particle and bulk densities of ordinary chondrite falls are 3.6 and 3.26 $\mathrm{g} / \mathrm{cm}^{3}$, respectively, which are higher than those for any carbonaceous chondrites save the CB chondrites. The average porosity is $9.5 \%$ for Hs and LLs, and 8.0 for Ls (falls only), which are generally much lower than those for carbonaceous chondrites. The average magnetic susceptibilities for ordinary chondrite falls range from 4.13 (for LLs) to $5.30 \log 10^{-9} \mathrm{~m}^{3} / \mathrm{kg}$ (for $\mathrm{Hs}$ ), in the same range as the carbonaceous chondrites. For ordinary chondrites, Consolmagno et al. (2013) found an average heat capacity to be $494 \mathrm{~J} / \mathrm{kg} \bullet \mathrm{K}$ at temperatures between 77 and 294K. However, these properties were all measured on relatively sturdy rocks which survived atmospheric entry, and the properties of asteroid regoliths should be very different, and are still poorly understood.

\subsubsection{Primary (Nebular) Characterisitcs of the Ordinary Chondrites}


Ordinary chondrites (OCs) are silicate-rich meteorites composed predominently of olivine and low-calcium pyroxene that are by far the most abundant meteorites ( $80 \%$ of all falls; Hutchison, 2004) though not necessarily the amost abundant asteroid material. As described earlier, they were subdivided back in the 1960s into three groups (H, L and LL) based on variations in bulk composition, such as molecular ratios $(\mathrm{FeO} /(\mathrm{FeO}+\mathrm{MgO}))$ in olivine and pyroxene and the ratio of metallic Fe to total Fe (Hutchison, 2004). However, in the intervening 40 years, as data from ordinary chondrites have steadily accumulated, the compositional gaps between the three ordinary chondrite groups have disappeared - there is a continuum between them (Figure 2.1) (Vernazza et al., 2015).

$\mathrm{H}$ chondrites have the highest total abundance of $\mathrm{Fe}$ (and the highest $\mathrm{Fe} / \mathrm{Si}$ atomic ratio) among OCs. They are also the most reduced OCs, their iron being mostly in metal. Equilibrated $\mathrm{Hs}$ have the lowest silicate $\mathrm{FeO}$ contents. LL chondrites contain the least total Fe, corresponding to the lowest $\mathrm{Fe} / \mathrm{Si}$ atomic ratios, and are the most oxidized $\mathrm{OCs}$, accordingly with the least metal and with the highest silicate $\mathrm{FeO}$ contents when equilibrated. $\mathrm{H}$ and LL chondrites also differ in terms of oxygen isotopes, with LL chondrites containing a the lowest ${ }^{16} \mathrm{O}$ relative to the other two isotopes $\mathrm{H}$ chondrites containing the highest.

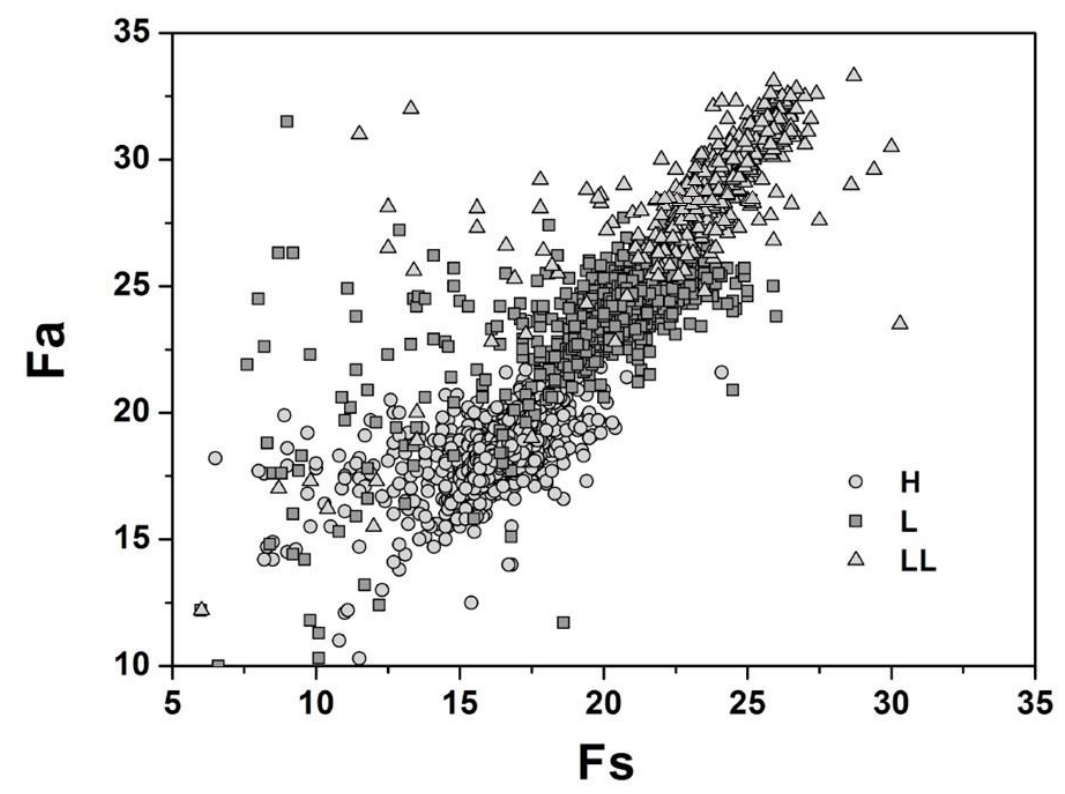

Figure 2.1 Comparison between the mineral chemistry of H, L, and LL olivines. Each datapoint represents the average olivine from a single meteorite. After Nakamura et al., 2011.

\subsubsection{Chondrules in Ordinary Chondrites:}

Unequilibrated ordinary chondrites (UOCs) are mainly aggregates of chondrules ( 60 $80 \%$ of the volume), metal and sulfide grains set in fine-grained matrix (10-15 vol\%), the latter consisting mainly of olivine (Hutchison 2004). The most abundant chondrule types in UOCs, in approximate order of abundance are porphyritic olivine-pyroxene, porphyritic olivine, porphyritic pyroxene, radial pyroxene, compound, barred olivine and granular. In general chondrules sizes in OCs range up to about $1 \mathrm{~mm}$, but rare individuals up to golf-ball sized have been reported occasionally (Prinz et al., 1988). With increasing thermal metamorphism 
chondrule outlines become progressively blurred and finally disappear. Similarly, with increasing degrees of impact shock chondrules are progressively abraded and finally destroyed.

Chondrule compositions have bulk compositions close to that of the solar photosphere, except for depletions of elements more volatile than $\mathrm{Fe}$, which are typically more abundant in the matrix. This complementarity between chondrules and matrix bulk composition has been the subject of much discussion (Huss et al., 2005). The major minerals in ordinary chondrite chondrules are olivine and low-Ca pyroxene, with minor glassy mesostasis rich in $\mathrm{Ca}, \mathrm{Al}, \mathrm{Na}$, and $\mathrm{K}$. Fe-Ni metal and troilite are also present in some chondrules. Chondrules with more reduced silicates are called type I. The ferromagnesian silicates in these type I chondrules have $\mathrm{Fe} /(\mathrm{Fe}+\mathrm{Mg})<0.5$ to 1 , and generally contain metallic $\mathrm{Fe}, \mathrm{Ni}$ droplets, which are less abundant in the more oxidized type II chondrules. As one would imagine, the type II chondrules contain olivine and pyroxene with large amounts of $\mathrm{FeO}$, and are presumed to have formed in a more oxidized nebular time or place (Gooding \& Keil 1981, Cuzzi \& Alexander 2006).

Chondrules show a wide range of textures reflecting diverse chemical compositions, peak temperatures $\left(1400-1850^{\circ} \mathrm{C}\right)$, formation regions, chiondrule-chondrule impact events, reheating, and cooling histories. Those heated above their liquidus temperatures show fine-grained, cryptocrystalline textures, whereas those heated below the liquidous preserve some crystal nuclei resulting in porphyritic textures. Decades of chondrule forming lab simulations indicate cooling rates of $10-1000{ }^{\circ} \mathrm{C} /$ hour (Hewins et al. 2005). A significant proportion (at least $15 \%$ ) of chondrules have relict grains that must be fragments or grains from earlier generations of chondrules or refractory objects such as calcium aluminum-rich inclusions (CAI) or amoeboid olivine aggregates (AOAs). Chondrule formation was thus a repetitive process that involved mixing of materials from diverse sources over a few million years (Russell et al. 2005, Cuzzi and Alexander, 2006). Type I chondrules ordinary chondrites have igneous rims of forsterite and enstatite, which suggests that chondrite matrix may be a mixture of silicate dust grains from all the regions where the associated chondrules formed.

\subsubsection{Refractory Inclusions in Ordinary Chondrites}

As described above, refractory inclusions in chondrites are composed mainly of minerals that crstallized first from the nebular cloud of gas (MacPherson 2003, MacPherson et al. 2005). There are two basic types: CAIs that are composed of refractory Ca-Al-Ti minerals, such as corundum, hibonite, grossite, perovskite, spinel, melilite, Al-diopside (which is still called fassaite by many, though this term was invalidated 40 years ago), and anorthite, and the AOSs that are composed of various proportions of forsterite, Fe-Ni metal, spinel, anorthite, and Al,Ti-pyroxene (MacPherson 2005, MacPherson et al. 2005). Refractory materials in ordinary choindrites are not nearly as abundant, nor have they received the same level of study, as those in carbonaceous chondrites.

\subsubsection{Fine-Grained Materials in Ordinary Chondrites}

The fine-grained material in UOCs is relatively poorly understood, although it is known to be dominated by olivine with a rather large range of composition (Frank et al., 2014). Where closely examined, as in Semarkona, the matrix appears to have experienced significant alteration (Huss et al., 2005). In addition to the indigenous solar materials, UOC matrix includes a 
sprinkling of presolar grains at the ppm to ppb abundance, as does the matrix in all other unequilibrated chondrites (Tonotani et al., 2006).

Beyond metamorphic grade 4 the fine-grained materials in ordinary chondrites has been thoroughly recrystallized to coarse-grained olivine and pyroxene.

\subsubsection{Aqueous alteration in Ordinary Chondrites.}

There are a few, predominantly unequilibrated, type 3 OCs that show evidence of having experienced significant degrees of aqueous alteration, involving either liquid or vapor phase water (Alexander et al., 2012). Secondary assemblages resulting from aqueous alteration include saponite adjacent to assemblages of the carbides cohenite $\left((\mathrm{Fe}, \mathrm{Ni}){ }_{3} \mathrm{C}\right)$ and haxonite $\left((\mathrm{Fe}, \mathrm{Ni})_{23} \mathrm{C}_{6}\right)$ and magnetite in the matrix of several LL3 chondrites (Hutchison et al., 1987; Alexander et al., 1989; Krot et al., 1997; Johnson et al., 2016). Krot et al. (1997) proposed that carbide-magnetite assemblages formed as the result of hydrothermal alteration of metallic $\mathrm{Fe}$ in metal-troilite nodules by a C-O-H bearing fluid on OC parent bodies. This alteration resulted in:

i. $\quad$ carbidization of $\mathrm{Fe}-\mathrm{Ni}$ metal, probably by $\mathrm{CO}$ gas (e.g., $15 \mathrm{Fe}(\mathrm{s})+4 \mathrm{CO}(\mathrm{g})=$ $\mathrm{Fe}_{3} \mathrm{C}(\mathrm{s})+\mathrm{Fe}_{3} \mathrm{O}_{4}(\mathrm{~s})$ or $\left.3 \mathrm{Fe}(\mathrm{s})+2 \mathrm{CO}(\mathrm{g})=\mathrm{Fe}_{3} \mathrm{C}(\mathrm{s})+\mathrm{CO}_{2}(\mathrm{~g})\right)$, and

ii. oxidation, probably by $\mathrm{H}_{2} \mathrm{O}$ gas (e.g., $3 \mathrm{Fe}(\mathrm{s})+4 \mathrm{H}_{2} \mathrm{O}(\mathrm{g})=\mathrm{Fe}_{3} \mathrm{O}_{4}(\mathrm{~S})+4 \mathrm{H}_{2}(\mathrm{~g})$ ).

The C-O-H bearing fluids may have been derived from ices, adsorbed gases, or hydrated minerals. These fluids were possibly released during metamorphism and transported through zones of high permeability. The carbon monoxide was probably produced from the reaction of carbon compounds (e.g., hydrocarbons) with water vapor or magnetite. Carbide-magnetite assemblages have only been found in LL3 chondrites, possibly indicating their more extensive oxidation and/or aqueous alteration (Krot et al., 1997). An example of a vein of phyllosilicates (saponite in this case) within a L3 chondrite is shown in Figure 2.2. 


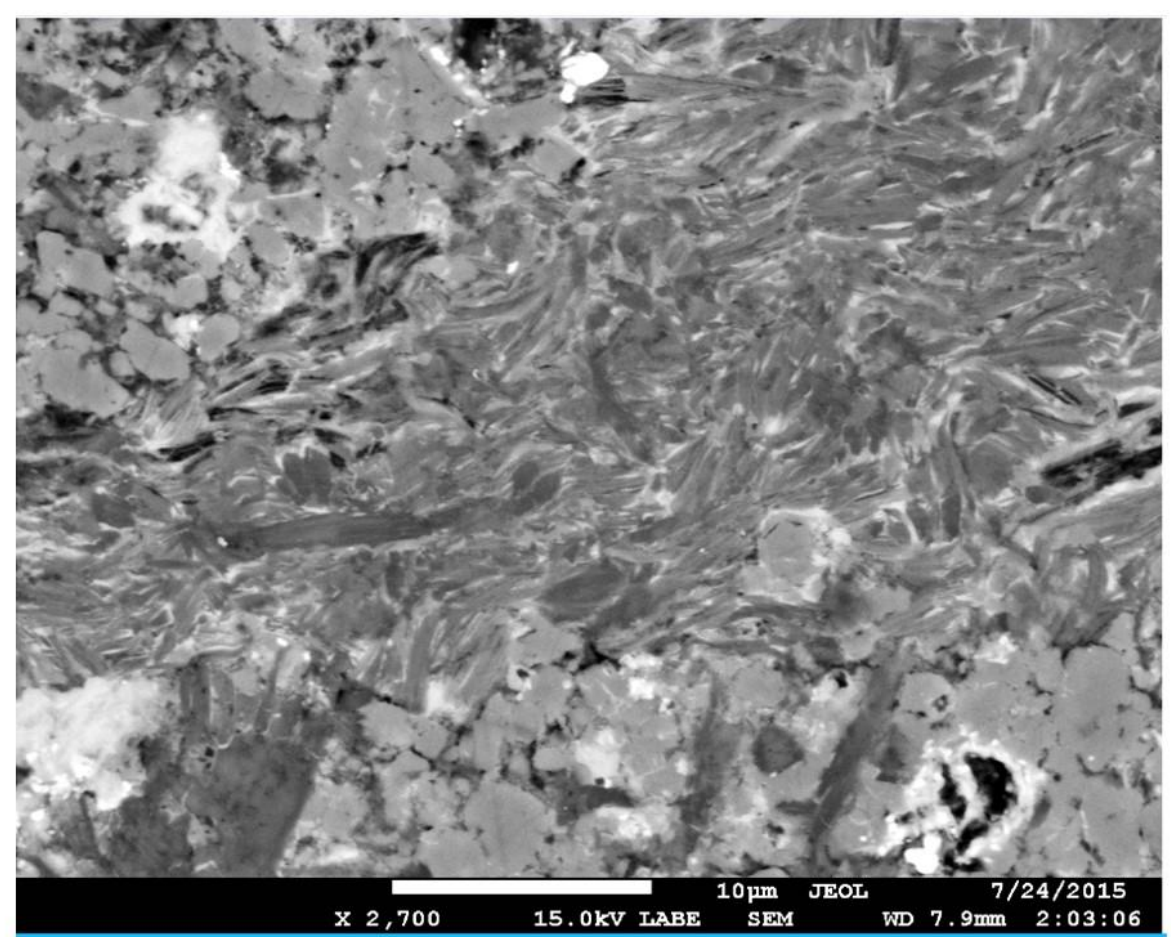

Figure 2.2. BSE image of a vein of saponite cutting across the L3 chondrite NWA 6169, from left to upper right.

\subsubsection{Impact-related Processes and Brecciation in Ordinary Chondrites.}

The first shock scale for meteorites was devised for ordinary chondrites (Stöffler et al., 1991), since the vast majority of them exhibit effects from impact shock. A summary of the petrographic evidence (under plane polarized light) and associated peak shock levels (S1= Shock level 1, etc.) are as follows:

$\mathrm{S} 1$ : completely unshocked (up to $5 \mathrm{GPa}$ ): No petrographic evidence for shock

S2: very weakly shocked (5-10 GPa); uneven darkening of olivine; planar and irregular fractures (aside from cleavage)

S3: weakly shocked (15-20 GPa); irregular fractures in olivine; dark shock veins and some melt pockets (isotropic under polarized light)

S4: moderately shocked; (30-35 GPa); weak planar fracturing of olivine; some pockets of melted material, dark interconnected shock veins

S5: strongly shocked (45-55 GPa); very strong planar fracturing and planar deformation features in olivine; alteration of plagioclase into maskelynite; formation of dark melt veins

S6: very strongly shocked (75-90 GPa); olivine has recrystallized, with local alteration to the high-pressiure form ringwoodite, and shock melting of plagioclase to a glass

At still greater shock pressures the entire meteorite can have melted, and is refered to as an impact melt. 
Alan Rubin (Rubin, 2003, 2004) has proposed that shock is the major cause of thermal metamorphism in ordinary chondrites, and in fact there is a rough correlation between petrographic grade and shock level for these meteorites, with some of the more obvious shock features having been destrpoyed by subsequent thermal annealing. However this contention is controversial.

About $20-30 \%$ of all ordinary chondrites are fragmental breccias composed of various petrologic types, impact melt products, and occasional xenolithic material (Bischoff et al., 2006). Regolith breccias, which are fragmental breccias containing a small fraction of grains with solarwind gases that were exposed on the surface of the parent asteroid. These breccias are interpreted to indicate that each OC parent body contains both unequilibrated and equilibrated petrologic type material and that type 3 to 6 material can be found in a single body.

Clues to the recent impact history of the OC parent bodies can be obtained from their cosmic-ray exposure ages, which range from 1-100 Myr (Herzog and Caffee, 2014). The range of cosmic ray exposure ages for OCs are dominated by a few peaks showing that the production of meteorites is dominated by a small number of impact events on a limited number of parent bodies (Herzog and Caffee, 2014). H, L, and LL chondrites each have different peaks in cosmic ray exposure ages, but petrologic types within any group have the same peaks, which means that the ordinary chondrite parent bodies were well mixed by impacts. In other words the OC parent asteroids, preseumed to be the S-type asteroids, no longer have structural zoning with separate petrologic types - for example if these asteroids originally had a layered structure with the least metamorphosed (type 3) material (type 6) near the surface and most metamorphosed at the core (the "onion shell structure"), this layering has not survived to the present day (Vernazza et al., 2015).

\subsubsection{Thermal Metamorphism in Ordinary Chondrites}

As described above, a petrologic classification scheme (Van Schmus \& Wood 1967) for OCs was developed with the H, L and LL groups are further subdivided into groups from 3 to 6 according to degree of thermal metamorphism. The relatively unmetamorphosed type $3 \mathrm{~s}$ (unquilibrated ordinary chondrites, or UOCs) are further divided into 3.0-3.9 based on induced thermoluminescence properties (Sears et al., 1980), textures of opaque minerals (Bourot-Denise et al., 1997) structure of organics (Bonal et al., 2006; Bonal et al., 2007), and olivine Cr content (Grossman and Brearley, 2005).

Progressive thermal metamorphism has caused recrystallization and coarsening of all matrix and chondrule phases, including olivine, pyroxene, metal sulfides, phoisphates. Glassy mesostasis is crystallized into plagioclase, which also progressively coarsens. A few UOCs, especially LL3s, contain rare phyllosilicates (maninly saponite) in matrix. This is usually associated with magnetoite and Fe-Ni carbides, which are all (presumed to have formed simultaneously (Alexander et al., 1989; Krot et al., 1998). Any phyllosilicates which may have existed in UOCs has recrystallized to olivine and pyroxene by type 4.

\subsubsection{Boulder scale: Proposed shape-strength relationships in ordinary chondrites}

Only limited information about the boulder-scale properties of the OC parent bodies can be obtained from studying meteorites or from telescopic observations. Here, we call blocks larger ( $\geq$ several meters) positive relief features "boulders." Meteorites tend to be small. The 
dimensions of the largest OCs discovered on the earth are less than $\sim 2$ meters across (the main mass of the Jilin H5 chondrite). Hence, we are not able to investigate directly OCs greater than several meters on the earth. Ground-based telescopic imaging does not have high enough resolution to directly observe the boulders on asteroidal surfaces.

To date, the analysis of the images of boulders on asteroids acquired by space probes is the only way to investigate OC-like boulders greater than $\sim 2$ meters. The Galileo (NASA), Near Earth Asteroid Rendezvous (NEAR)-Shoemaker (NASA), Hayabusa (JAXA) spacecraftrendezvoused with S-type asteroids, Gaspra, Ida, Eros (; Veverka et al., 2000) and 25143 Itokawa $(0.535 \times 0.294 \times 0.209$ km; Fujiwara et al., 2006), respectively. Returned 25143 Itokawa samples unequivocally demonstrated that OCs originate from S-type asteroids (e.g., Nakamura et al., 2011; Yurimoto et al., 2011). Therefore, in order to understand the formation and properties of OCs, it is necessary to examine the regolith and boulders on S-type asteroid surfaces.

Studies of 433 Eros and 25143 Itokawa revealed a substantial regolith and boulders greater than several meters on these asteroid surfaces (for more details about asteroidal missions see Clark et al., this volume). In addition to 433 Eros and 25143 Itokawa, the recent flyby of S-type asteroid 4179 Toutatis by Chang'e-2 spacecraft yielded $45 \%$ images of its surface (Huang et al., 2013). The size of 4179 Toutatis $(\sim 4.6 \times 2.3 \times 1.9 \mathrm{~km}$; Huang et al., 2013) is intermediate between 433 Eros and 25143 Itokawa. In the following paragraphs, we will discuss features of the boulders on these S-type asteroids, i.e. 25143 Itokawa, 4179 Toutatis and 433 Eros in order of increasing asteroid size. Although the Galileo probe also visited S-type asteroid 951 Gaspra and observed boulders are also observed on S-type asteroid 243 Ida, we exclude these boulders due to the deficiency of the number of measurement from the statistical point of view.

The shape of 25143 Itokawa resembles a sea otter composed of a smaller, rather round head and a larger oval body (Fujiwara et al., 2006). Its surface is covered with numerous boulders despite its small mass and gravity, which are very small compared with those of the other asteroids previously observed from spacecraft (Saito et al., 2006). The surface of 25143 Itokawa is separated into "rough terrains", which occupy about $80 \%$ of its surface with large gravel and boulders, and "smooth terrains" covered by fine-grained $(>\sim 1 \mathrm{~mm}$ ) regolith particles (Fujiwara et al., 2006; Saito et al., 2006; Yano et al., 2006). Close-up images indicate that the rough terrains are completely devoid of all particles smaller than $1 \mathrm{~cm}$ in size (Yano et al., 2006). The boulder shapes are continuously being modified by impact erosion, and exfoliation by heating/freezing cycles. The smooth terrains correspond to low-gravitational potentials and are generally flat, such terrains are called "ponds" - see below. The boundaries between the rough and smooth terrains are relatively sharp. Thus, most boulders on 25143 Itokawa are found in the rough terrains. In the smooth terrains, boulders seem to be buried by regolith particles. Overall, the boulders appear to be uniformly spread over the entire surface because the rough terrains occupy about $80 \%$ of 25143 Itokawa's surface as mentioned before.

The shape of 4179 Toutatis resembles that of a ginger root made up of a smaller, angular head and a larger, angular body (Huang et al., 2013). More than 200 boulders were identified over the imaged area $\left(\sim 6.68 \mathrm{~km}^{2}\right)$ (Jiang et al., 2015). The two largest boulders $(>50$ $\mathrm{m})$ are located in the neck region combined with head and body (Jiang et al., 2015). The boulders appear to be relatively uniformly spread over the entire surface; although the observed number of the boulders is limited.

433 Eros is an elongated asteroid. Most boulders are located near and within Shoemaker crater, and are generally considered to originate from that crater (e.g., Thomas et al., 2001). 
Nearly half of the volume of all boulders larger than $15 \mathrm{~m}$ is within Shoemaker crater. Boulders outside this crater are concentrated near the equator, and are relatively depleted in some longitudes (Thomas et al., 2001). The morphology of these boulders ranges from angular, to fractured, to disaggregated (Robinson et al., 2002). One striking feature first observed on Eros was asteroid ponds, formed through dust remobilization and seismic shaking (Veverka et al., 2001a; Robinson et al. 2001; Cheng et al., 2002). These ponds characteristically have very smooth surfaces and are found mainly within craters. Occasionally boulders or outcrops project through the pond surfaces. Though level to a first approximation (and corresponding well to the gravitational equipotential field, they can exhibit small, steep-walled, fault- or slump-like features, which suggests that the pond deposits can be indurated to some degree (Veverka et al., 2001a). The ponds are distributed preferentially in low latitudes, near the ends of the elongated asteroid. These latitudes coincide with surfaces that spend the most time near Eros' terminator, as well as being the regions with the lowest surface gravity (Cheng et al., 2002). The presence of boulders with different morphologies suggests that multiple lithologies may be represented on the surfaces of 433 Eros and 25143 Itokawa. From the close-up images, there are two major types of boulders on both asteroids: one has rounded edges and corners (rounded boulders), while the other has angular edges and corners (angular boulders) (e.g., Noguchi et al., 2010). The surface features of the rounded boulders suggest that they have hardness heterogeneity and that some may be breccias. The angular boulders appear to be more resistant to impact disruption than the rounded ones, which may be due to a difference in lithology. Such morphological features of boulders is compatible with the characteristics of LL chondrites - i.e. more than $60 \%$ of LL chondrites are breccias (Binns, 1967).

To date, the source region of L chondrites are considered to be the Gefion family based on dynamical and compositional aspects (Nesvorny et al., 2009; Vernazza et al., 2014, 2015), and that of most LL chondrites are considered to be Flora family (Nesvorny et al., 2008; Vernazza et al., 2014, 2015). Unfortunately, the source of $\mathrm{H}$ chondrites is currently unknown (Vernazza et al., 2015).

As a whole, the range of observed slopes on 25143 Itokawa, 4179 Toutatis, and 433 Eros is comparable to that found for the boulder distributions of lunar impact craters, which cluster between -3 and -4 (e.g., Bart and Melosh, 2010). The slope index of the distributions are $-3.1 \pm$ 0.1 for the 5-40 m boulders on 25143 Itokawa (Michikami et al., 2008), -4.4 \pm 0.1 for the 20-61 $\mathrm{m}$ boulders on 4179 Toutatis (Jiang et al., 2015), and -3.2 for the 15-80 m boulders on 433 Eros (Thomas et al., 2001). The slope of -4.4 for 4179 Toutatis boulders is steeper than those for 25143 Itokawa and 433 Eros, which may imply a different preservation state or diverse formation scenarios (e.g., Jiang et al., 2015). For instance, Jiang et al. (2015) suggested that the boulders are comminuted by impacts after the final assembly of asteroid 4179 Toutatis, and the size distribution become steep because large boulders may be destroyed but not replenished. However, in general, the slope index also changes depending on the measurement method (e.g., Saito et al., 2006; Mazrouei et al., 2014) and the terrain properties (e.g., Bart and Melosh, 2010), although the differences between the models is relatively minor.

The low gravity of 25143 Itokawa, 4179 Toutatis, and 433 Eros result in the movement of boulders (and regolith particles) after the formation. On 25143 Itokawa, the larger-sized gravels tend to overlie the smaller particles and are aligned with directions coincident with the local gravity slope (Miyamoto et al., 2007). The positions and orientations of all the particles indicate that they are stable against local gravity and that gravity induced the migrations (Miyamoto et al., 2007). Relatively small boulders (gravels) would be redistributed by global vibration, which 
was caused by repeated impacts on the surface. Such a mechanism is known as seismic shaking, as proposed to explain the paucity of small craters on 433 Eros (Richardson et al., 2004).

In a more detailed study, Tancredi et al. (2015) found that the size distribution of the boulders on 25143 Itokawa is correlated with the total potential and the surface gravity: large boulders are concentrated in the "head" and "bottom" regions of higher potential (low surface gravity), and small boulders are located in the Muses-C region of lower potential (high surface gravity). Meanwhile, in the regions of high potential (low surface gravity), Tancredi et al. (2015) obtained a shallower slope in the size distribution, shifted towards the large boulders. Tancredi et al. (2015) also confirmed that there is a size segregation that is correlated with the gravity field, which can be explained by the Brazil nut effect. In addition, a recent study shows that the segregation may be caused by a simple and unexplored effect: pebbles accreting onto the asteroid rebound from boulders, but sink into pebbly regions (Shinbrot et al., 2017). As a result, there was a global relocation of boulders, with large ones going into the high potential regions and small ones into the low potential ones.

In average, the number densities of boulders $>20 \mathrm{~m}$ on the surfaces of 25143 Itokawa $\left(\sim 15 / \mathrm{km}^{2}\right)$ and 4179 Toutatis $\left(\sim 17 / \mathrm{km}^{2}\right)$ are greater than that on 433 Eros $\left(\sim 2-20 / \mathrm{km}^{2}\right.$; median of the order of several $/ \mathrm{km}^{2}$. The difference would show that the formation of 433 Eros boulders differs from that of 25143 Itokawa and 4179 Toutatis boulders. Most boulders on the surface of relatively large asteroids are considered to be connected to source craters, like the Shoemaker crater on 433 Eros, the Stickney crater on Phobos and the central crater on 21 Lutetia, while the distribution of boulders on 25143 Itokawa and 4179 Toutatis (relatively small asteroids) likely results from processes involved in their re-accretion following the catastrophic disruption of the parent body (e.g., Michikami et al., 2008; Jiang et al., 2015). In fact, the ratios of the total volume of the boulder to the total excavated volume of the crater are $\sim 25 \%$ for 25143 Itokawa (Michikami et al., 2008), 10 \% for 4179 Toutatis (Jiang et al., 2015) and $\sim 0.4 \%$ for 433 Eros (Thomas et al., 2001). In addition, the largest boulder sizes of 25143 Itokawa and 4179 Toutatis are $40 \mathrm{~m}$ and $61 \mathrm{~m}$ in diameter, respectively, and are significantly larger than that estimated from the empirical relationship between the largest boulder size and the crater, which are applied for the Moon, Phobos, Deimos, 243 Ida and 433 Eros (Lee et al., 1996; Thomas et al., 2001). Moreover, the global mapping of boulders on 25143 Itokawa and 4179 Toutatis show that there is no apparent correlation between the locations of boulders and craters. These facts prove that the boulders on 25143 Itokawa and 4179 Toutatis cannot solely be the product of craters, and originated from the catastrophic disruption of their larger parent bodies.

25143 Itokawa is probably the product of the reaccumulation of small fragments (mega-blocks and boulders) generated by the catastrophic disruption of a larger parent body. The following lines of evidence support this hypothesis: (1) numerical simulation (e.g., Michel and Richardson, 2013), (2) the mineral chemistry of the 25143 Itokawa returned particles (Nakamura et al., 2011), (3) abundant boulders showing textures of possible breccias (Noguchi et al., 2010), and (4) the shape of the boulders on 25143 Itokawa (Michikami et al., 2016). This estimation is consistent with the formation of regolith breccias during catastrophic disruption of large (on the order of $10^{2} \mathrm{~km}$ ) asteroids (Stöffler et al., 1988). Therefore, the formation of boulders on asteroidal surfaces would depend strongly on the size of body. That is, in the case of relatively large asteroids (greater than critical size $\sim 10 \mathrm{~km}$ ) like 433 Eros, most boulders are primary produced by the large crater formation. In contrast, in relatively small asteroids (smaller than several $\mathrm{km}$ ) like 25143 Itokawa and 4179 Toutatis, most boulders are primary produced by the catastrophic disruption of a larger parent body. 


\subsubsection{Regolith-grain scale: Shape and Comminution Processes in OCs}

Regolith particles recovered from the S-type asteroid 25143 Itokawa by the Hayabusa mission are not only the first samples recovered from an asteroid, but also the second extraterrestrial regolith (the first being the Moon). The sizes of the regolith particles range from 3 to $180 \mu \mathrm{m}$, but most < $10 \mu \mathrm{m}$ (Nakamura et al., 2011). The three-dimensional (3-D) microstructure of fourty-eight regolith particles from 25143 Itokawa (sizes from 10 to 100 $\mu \mathrm{m}$ ) were examined by synchrotron microtomography at Spring-8 (Tsuchiyama et al., 2014). Hence, it is possible to make a comparison between the regolith particles brought back to Earth and the close-up images from the surface of 25143 Itokawa obtained by the Hayabusa spacecraft.

Regolith depth ranges from asteroid to asteroid and within individual astroids. On 25143 Itokawa, the regolith depth in the smooth regions is estimated to be approximately $2.5 \mathrm{~m}$ (Cheng et al., 2007; Barnouin-Jha et al., 2008). 433 Eros has a widespread unconsolidated regolith of depths that are typically several tens of meters in thickness, but not uniform over the surface (Barnouin-Jha et al., 2001; Veverka et al., 2001a; Cheng, 2002; Robinson et al., 2002, Murdoch et al., 2015). The heterogeneity of the regolith depth distribution on 433 Eros is probably caused by the asymmetric nature of crater ejecta blankets (e.g., Murdoch et al., 2015).

The grain size of regoliths varies from asteroid to asteroid. The size distribution of the 25143 Itokawa regolith particles ranging from $\sim 10-100 \mu \mathrm{m}$ acquired from a microtomography has a slope index of about -2 (Tsuchiyama et al., 2011), which is more gradual than that (-3) of the 25143 Itokawa boulders (e.g., Michikami et al., 2008), indicating a lower abundance of 10-100 $\mu \mathrm{m}$-sized regolith particles compared to millimeter- to centimeter-sized regolith (Tsuchiyama et al., 2011, 2014). This is compatible with the close-up images of regolith taken by the Hayabusa spacecraft (Yano et al., 2006). Most grains in the smooth terrains on 25143 Itokawa are larger than those observed in the close-up view of 433 Eros' surface. There is a strong depletion of fine grains on 25143 Itokawa compared with 433 Eros. That is, 25143 Itokawa's regolith appears to be dominated by grains > $1 \mathrm{~mm}$ in size (Yano et al., 2006; Miyamoto et al., 2007), while 433 Eros's ponds (smooth terrains on the surface) appears to be covered with fine grains at least $<1$ $\mathrm{mm}$ in size (Veverka et al., 2001b). Minimum grain sizes should be dependent on the asteroid masses, but in fact these sizes are just estimates.

Variations in regolith grain size across asteroids may be the result of different formational process and differences in asteroidal diameters. For example, the shape distribution of the 25143 Itokawa regolith particles (Tsuchiyama et al., 2011, 2014) is similar to that of laboratory experimental fragments (e.g., Fujiwara et al., 1978). Thus, Tsuchiyama et al. (2011, 2014) suggested that the Itokawa regolith particles are probably the results of mechanical disaggregation, primarily as a response to impacts. Depletions in regolith grains with diameters > $1 \mathrm{~mm}$ in 25143 Itokawa observed in remotely and in returned samples may have resulted from electrostatic levitation by solar radiation pressure (Lee, 1996; Scheeres, 2005). Micro impacts by interplanetary dust would have repeatedly fragmented the surface materials of 25143 Itokawa and most small fragments (fine regolith particles) would escape from the surface because the gravity of such a small asteroid is very low. Yoshikawa et al. (2015) suggested that fine regolith particles are gradually lost and the surface becomes covered with larger particles and boulders. As a probable explanation for the existence of fine regolith particles on 25143 Itokawa to date, Delbo et al. (2014) showed that thermal fragmentation induced by the diurnal temperature variations breaks up rocks larger than a few centimeters more quickly than do micrometeoroid impacts. As another explanation, large macroporosity of Itokawa body and armoring process would not only reduce the shock effect during impacts, but could also help produce fine regolith 
particles near the surface (Yoshikawa et al., 2015). In any event, these mechanisms would produce and/or preserve the regolith particles on small asteroids. Dombard et al. (2010) proposed that ponds on 433 Eros formed from thermally disaggregated and seismically flattened boulder material, under the assumption that repeated day/night cycling causes material fatigue that leads to erosion of the boulders. It appears likely that the same processes result in ponds on all S-type asteroids

As mentioned before, seismic shaking leads to erosion of the craters on 433 Eros and 25143 Itokawa. Small craters ( $<200 \mathrm{~m}$ in diameter) in 433 Eros are depleted in fine-grained regolith (Veverka et al., 2000). Also, many crater candidates on 25143 Itokawa are shallower than craters observed on the surface in other planetary bodies (Hirata et al., 2009). Seismic shaking on small bodies induces the movement of regolith particles; as a result, it would destroy small craters formed in such regolith (e.g., Chapman et al., 2002). It may be common small asteroids - like 433 Eros and 25143 Itokawa - that seismic shaking redistributed most regolith particles to regions of lower potential (e.g., Robinson et al., 2001; Tancredi et al. 2015).

\subsubsection{Terrestrial Weathering of Ordinary Chondrites}

Oxidation of primary metal and sulfides is the most conspicuous terrestrial weathering phenomenon of OC finds (e.g., Lee and Bland, 2004). Hydrolysis of anhydrous meteoritic silicates by either terrestrial carbonic acid (Velbel et al. 1991) or terrestrial sulfuric acid formed by oxidation of meteoritic sulfides produces evaporite efflorescences comprising sulfates and carbonates of the alkali and alkaline earth cations leached from primary OC meteorite phases (Jull et al. 1988; Velbel et al. 1991; Losiak and Velbel 2011). Such reactions are known both to proceed as post-recovery continuations of weathering initiated during pre-recovery weathering in Earth's natural environment, and in some instances even originate in curatorial and laboratory settings (Jull et al. 1988; Velbel et al. 1991; Losiak and Velbel 2011). Where the acid is sulfuric acid (Buddhue 1957), the acid anion can form a sulfate by combining with solubilized magnesium released by the acid-silicate hydrolysis reaction. The sulfate mineral formed by the combination of magnesium from olivine (or pyroxene) with the anion of dissociated sulfuric acid produced by oxidation of meteoritic sulfide becomes incorporated into evaporite minerals of terrestrial weathering origin, forming veins or efflorescences such as those widely recognized on stony meteorite finds (Losiak and Velbel 2011).

Ordinary chondrite falls are very numerous, so much material that is only minimally compromised by terrestrial weathering is available for comparison with mission data. Nevertheless, during recovery, prior to receiving, and during preliminary examination, some Hayabusa samples were briefly exposed to terrestrial atmosphere (Velbel, 2014). Some OC minerals (esp. olivine, sulfides, and Fe-Ni metal) are known to be among the most vulnerable to weathering in moist, oxidizing terrestrial environments. Oxidation of Fe host-minerals is ubiquitous in (1) naturally weathered OC finds, (2) falls exposed to terrestrial weathering for even a few decades of before recovery, and (3) OC falls recovered and curated promptly after recovery (Velbel, 2014 and primary references therein). All prerecovery oxidation, hydrolysis, hydration, and product-forming phenomena documented to affect $\mathrm{OC}$ finds have been documented to continue in OC samples in curatorial and laboratory settings, producing mineralogical and textural effects at scales easily discernable by electron microscopy, on timescales of decades (Velbel, 2014). Returned samples will be exposed to similar terrestrial conditions at times throughout sample processing, allocation, and examination (Velbel, 2014). 


\subsection{Enstatite chondrites and their counterpart asteroids.}

\subsubsection{Physical Properties of Enstatite Chondrites}

The physical properties of enstatite chondrites are significantly different from other chondrites, as it would be expected from their high metal contents. Macke et al. (2010) studied the physical properties of 23 enstatite chondrites, including both EL (Daniel's Kuil, Eagle, Hvittis, Jajh deh Kot Lalu, Khairpur, Pillistfer, Atlanta, Blithfield, Happy Canyon, Ilafegh 009, North West Forrest, NWA 2965, NWA 3132, and Yilmia) and EH (Abee, Adhi Kot, Indarch, Saint-Sauveur, St. Mark's, Bethune, Kota-Kota, Sahara 97096, and Sahara 97158) chondrites, which they found to have similar properties. Macke et al. (2010) noted that the properties of falls and finds were different from each other. For falls, they determined the average bulk density of enstatite chondrites to be $3.55 \mathrm{~g} / \mathrm{cm}^{3}$ (ranging from 3.15 to $4.10 \mathrm{~g} / \mathrm{cm}^{3}$ ) and the grain density 3.66 $\mathrm{g} / \mathrm{cm}^{3}$ (ranging from 3.45 to $4.17 \mathrm{~g} / \mathrm{cm}^{3}$ ). They found that the porosities range from 0 to $12 \%$, with the majority having porosities below 7\%. In general, there is much more variability on the physical properties of finds. Macke et al. (2010) measured the average magnetization to be $\log \chi$ $=5.47 \pm 0.04$. As expected, these are among the highest magnetizations for any chondrite group.

\subsubsection{Primary (Nebular) Characterisitcs of the Enstatite Chondrites}

\subsubsection{Chondrules in Enstatite Chondrites}

The unequilibrated enstatite chondrites (E3s) have abundant chondrules, including porphyritic olivine, porphyritic pyroxene, porphyritic olivine- pyroxene, radial pyroxene, cryptocrystalline and, rare, barred olivine. Porphyritic pyroxene is the most common textural type (Weisberg and Kimura, 2012). Because of the reduced nature of the E choncrite forming region, these chondrules are essentially all type I. Rare compound chondrules and igneous rims have also been reported from some type $3 \mathrm{E}$ chondrites (Rubin, 2010). Additionally, thin silica-rich rims on chondrules were described in some EH3 chondrites (Fagan et al., 2000).

\subsubsection{Refractory Inclusions in Enstatite Chondrites}

The refractory inclusions in E chondrites are relatively rare and small - reportedly 8$250 \mu \mathrm{m}$ in diameter, with an average diameter of $43 \mu \mathrm{m}$-Lin et al., 2003). However the inclusions show the typical structures of refractory inclusion from other chondrites. However, aside from their small sizes and low abundance, the refractory inclusions in E3 chondrites appear to have the same morphologies, textures, mineralogy, mineral chemistry, oxygen isotope ratios and magnesium isotope systematics as in ordinary and carbonaceous chondrites (Fagan et al., 2000, 2001; Weisberg and Kimura, 2012).

\subsubsection{Fine-grained Materials in Enstatite Chondrites}

The matrix of unequilibrated enstatite chondrites is poorly known, although there are some initial studies. As described by Weisberg et al. (2014), fine-grained matrix in the E3 chondrites is a distinct reduced mineral assemblage mainly of silica (both crystalline and non-crystalline) and enstatite. Rounded, glassy silica grains contain tiny sulfide, plagioclase, schreibersite, and enstatite 
crystals. EL3 matrix is dominated by enstatite and albitic plagioclase; silica abundance is lower and albitic plagioclase higher than in EH3 matrix. Both EH3 and EL3 matrix contains silica grains, unusual sulfides (Cr-rich troilite, heideite, etc), and Fe-Ni phosphides not reported in other chondrite matrix.

\subsubsection{Minerals of Enstatite Chondrites}

In general, the enstatite chodrites are among the most reduced of known astromaterials, and are almost the rarest (about $2 \%$ of modern falls -Grady, 2000). The are subdivided into EH and EL groups depending on their total amount of Fe-Ni metal: EH chondrites have greater than 25 vol. $\%$ metal, while EL have less than $25 \%$. They display a complete metamorphic trend from type 3 to 6 , analogous to the ordinary chondrites. As the name implies, enstatite is the dominant mineral in these meteorites, predominantly clinoenstatite in types 3, with disordered orthoenstatite progressively predominating with increasing metamorphism and recrystallized chondrules to grade 5. By grade 6 the orthoenstatite is essentially completely ordered (Hutchison, 2004). Crystalliation under reducing consitions has caused the enstatite composition to be almost entirely Fs $<1$, although some crystals with as much iron as Fs12 have been reported, and many contain high $\mathrm{Al}_{2} \mathrm{O}_{3}$ contents ( up to 14 wt.\%) (Weisberg and Kimura, 2012).

Due to their reduced origin, enstatite chondrites contain a fascinating variety of rare phosphides and sulfides, including Schreibersite $\left((\mathrm{Fe}, \mathrm{Ni})_{3} \mathrm{P}\right)$, Perryite $\left((\mathrm{Ni}, \mathrm{Fe})_{8}(\mathrm{Si}, \mathrm{P})_{3}\right)$, Florenskyite (FeTiP), Andreyivanovite (FeCrP), Oldamite ((Ca, $\mathrm{Mg}, \mathrm{Fe}) \mathrm{S})$, Niningerite, $\left(\left(\mathrm{Mg}, \mathrm{Fe}^{2+}, \mathrm{Mn}\right) \mathrm{S}\right)$, Daubreelite $\left(\mathrm{Fe}^{2+} \mathrm{Cr}_{2} \mathrm{~S}_{4}\right)$, Caswellsilverite $\left(\mathrm{NaCrS}_{2}\right)$, Sphalerite $((\mathrm{Zn}, \mathrm{Fe}) \mathrm{S})$, and Djerfisherite ( $\left.\mathrm{K}_{6} \mathrm{Na}(\mathrm{Fe}, \mathrm{Cu}, \mathrm{Ni})_{25} \mathrm{~S}_{26} \mathrm{Cl}\right)$ (Hutchison, 2004; Zolensky et al., 2008). There is also generally a high $\mathrm{Si}$ content in the metal (up to $3 \mathrm{wt} . \%$ ).

Because of the lack of iron in silicates in the enstatite chondrites, it is generally assumed that these derive from $\mathrm{E}$ or $\mathrm{M}$ type asteroids, whose reflectance spectra similarly lack evidence of iron-bearing silicates. It has been suggested that the asteroid 16 Psyche may be a parent for enstatite chondrites (Keil, 1989). No spacecraft has visited Psyche, but in 2017 the Psyche Mission to 16 Psyche was approved by NASA (for description of the mission see Clark et al., this volume). As currently planned, this spacecraft will launch in 2022 and arrive at Psyche in 2026. This would be an orbiting mission, not a sample return. It is interesting that the majority of the largest E-type asteroids show some spectral evidence for the $3 \mu \mathrm{m}$ feature indicative of water (Rivkin et al., 2002), but this result is debated. The debate could be settled by this mission.

\subsubsection{Hydrous Minerals in Enstatite Chondrites}

Zolensky et al. (2014) have proposed that rare, hydrated E chondrites are present as meteorites, though they are exceedingly rare. They describe that the Kaidun meteorite consists largely of such material (Figure 2.3). Here thoroughly-altered enstatite chondrite clasts are adjacent to complexly unaltered enstatite chondrite material. Even within single clasts, the alteration front can be abrupt. Carbide-containing metal is initially replaced by a poorly crystalline $\mathrm{Si}-\mathrm{Fe}$ phase, which in turn is replaced by serpentine. In the end the only indication of the original presence of metal is the residual carbides. In other enstatite chondrite lithogies in Kaidun (of uncertain type) original silicates and metal have been thoroughly replaced by an assemblage of authigenic plagioclase laths, calcite boxwork, and occasional residual grains of silica, $\mathrm{Cr}$-rich troilite, ilmenite, and rare 
sulfides including heideite. Fe and $\mathrm{S}$ have been largely leached from the rock. Again the accessory phases are the first clue to the original character of the rock, which was verified by $\mathrm{O}$ isotopes. It is fortunate that Kaidun displays every step of the alteration process. We do not know how frequently this occurred, but there may well have been "wet" E- or M-class objects at the time the terrestrial planets formed.
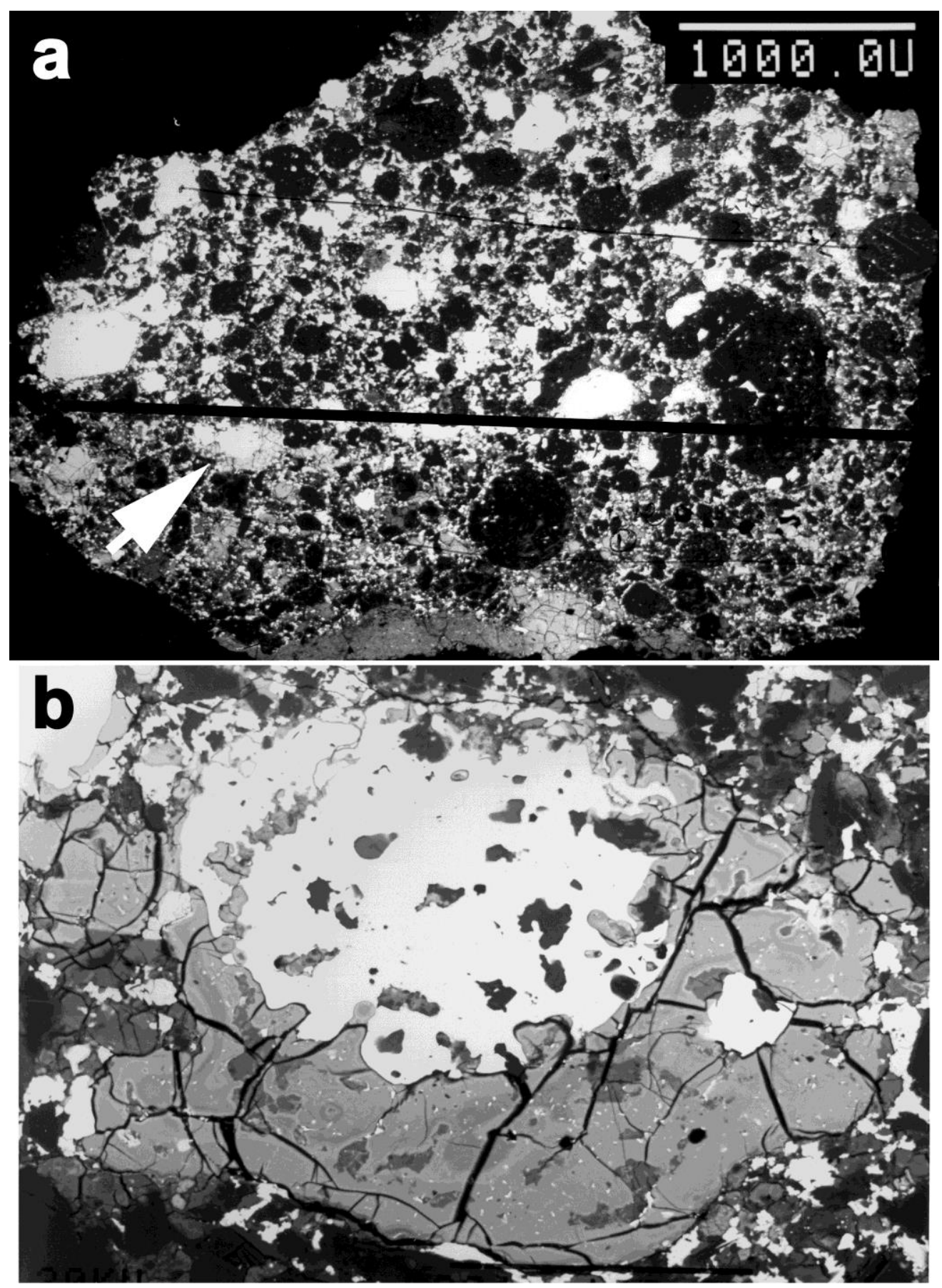

Figure 2.3 BSE images of a unique partially-aqueously altered EH lithology. (a) The entire clast. Above the black line the sample is pristine, below the line all phases are completely aqueously 
altered. (b) Closeup of altered chondrule [see arrow in (a)]. Metal and silicates are completely altered to iron-oxyhydroxides and silica gel (grey). Troilite has been transformed to pyrrhotite (white). After Zolensky and Ivanov (2004).

\subsection{Carbonaceous chondrites and their counterpart asteroids.}

\subsubsection{Regolith Properties of Carbonaceous Chondrites}

Regolith samples are poorly represented by meteorites, since recovered stones are the hardy survivors of a trip through earth's atmosphere. However, some solar-wind-gas-rich chondrites sample regolith materials, albeit tougher examples. Assuming that dust is levitated over the surface of all airless asteroids, the dust electric charge in the electric sheath produced by UV radiation can be calculated. Senshu et al. (2015) calculated the dust charge over the surface of S-type asteroid 433 Eros, and their calculations can be modified to apply to other asteroids. The micrometeorite flux on the surface of any asteroid can be estimated, as was done for the Martian moon Phobos, potentially a captured asteroid, which was estimated to be $10^{-16} \mathrm{~g} / \mathrm{cm}^{2} / \mathrm{sec}$ (Divine 1993). Surface temperatures from solar heating are significant (see section 2.5.4), probably varying from 60-350K, and diurnal variations will cause mechanical weathering of boulders. These variations can be estimated by 1 -dimensional thermal calculations (see section 2.5.4). They can also be interpolated from measurements of the lunar regolith.

C class asteroids frequently exhibit reflectance spectra consistent with thermally metamorphosed carbonaceous chondrites (Moskovitz, 2012), or a mixture of phyllosilicate-rich material along with regions where they are absent (Vilas, 2008). One particularly important example is near-Earth asteroid 162173 Ryugu, the target of the Hayabusa2 sample return mission (Moskovitz, 2012). In fact most spectra of Ryugu are featureless, suggesting a heterogeneous, incompletely dehydrated regolith (see section 2.1). One cause this dehydration may be impact shock melting. What evidence is there for significant shock melting in the otherwise very hydrated $\mathrm{CM}$ and $\mathrm{CI}$ chondrites? $\mathrm{CV}$ and $\mathrm{CM}$ impact melt clast have recently been recognized in CV and regolithic howardite host rock (Lunning et al., 2016). When they are carefully interrogated, CM and CI chondrite regolith breccias all appear to contain agglutinate grains, which exhibit melted matrix grading into merely desiccated phyllosilicates (Zolensky et al., 2014, 2015). The agglutinate glass is partially devitrified into normally-zoned olivine crystals. It is interesting that these glasses have partially devitrified to olivine, in contrast to the situation for lunar agglutinates (Lindsay Keller, personal communication, 2014). This could be due to a higher olivine normative composition, and the difficulty of quenching a liquid with an almost pure olivine composition (Gary Lofgren personal communication, 1999).

Shock melted C2 chondrite materials are widespread in the unique Kaidun meteorite breccia lithologies (mainly $\mathrm{C}$ and $\mathrm{E}$ chondrites), some of which have experienced post-shock aqueous alteration, which severely masks the evidence for shock (Zolensky and Ivanov, 2003; Zolensky et al., 2014). In these melt-vein samples, zoned acicular olivine and plagioclase (potassium-rich anorthoclase) crystals have nucleated into pyroxene-composition mesostasis. In one example from a CM1 lithology in Kaidun (Fig. 2.4a), the entire assemblage has been altered to phyllosilicates, which preserve most aspects of the compositions and even the zoning. Present in every $\mathrm{C}$ chondrite regolith breccia (even CI chondrites) are 10-100 $\mu \mathrm{m}$-sized beads of glass or phyllosilicates, which are likely a product of impact (Fig.2.4b). Masses of melted sulfides also 
record a flash heating events. For example, many troilite masses in the Jbilet Winselwan CM2 chondrite exhibit flash melting textures (Fig 2.4c-d), displaying inward injected matrix silicate grains (Zolensky et al., 2016). The degree of shock heating varies considerably in this particular meteorite. In addition to the melted sulfides, this meteorite also contains partially equilibrated olivine aggregates and chondrules, matrix phyllosilicates transformed into olivine.

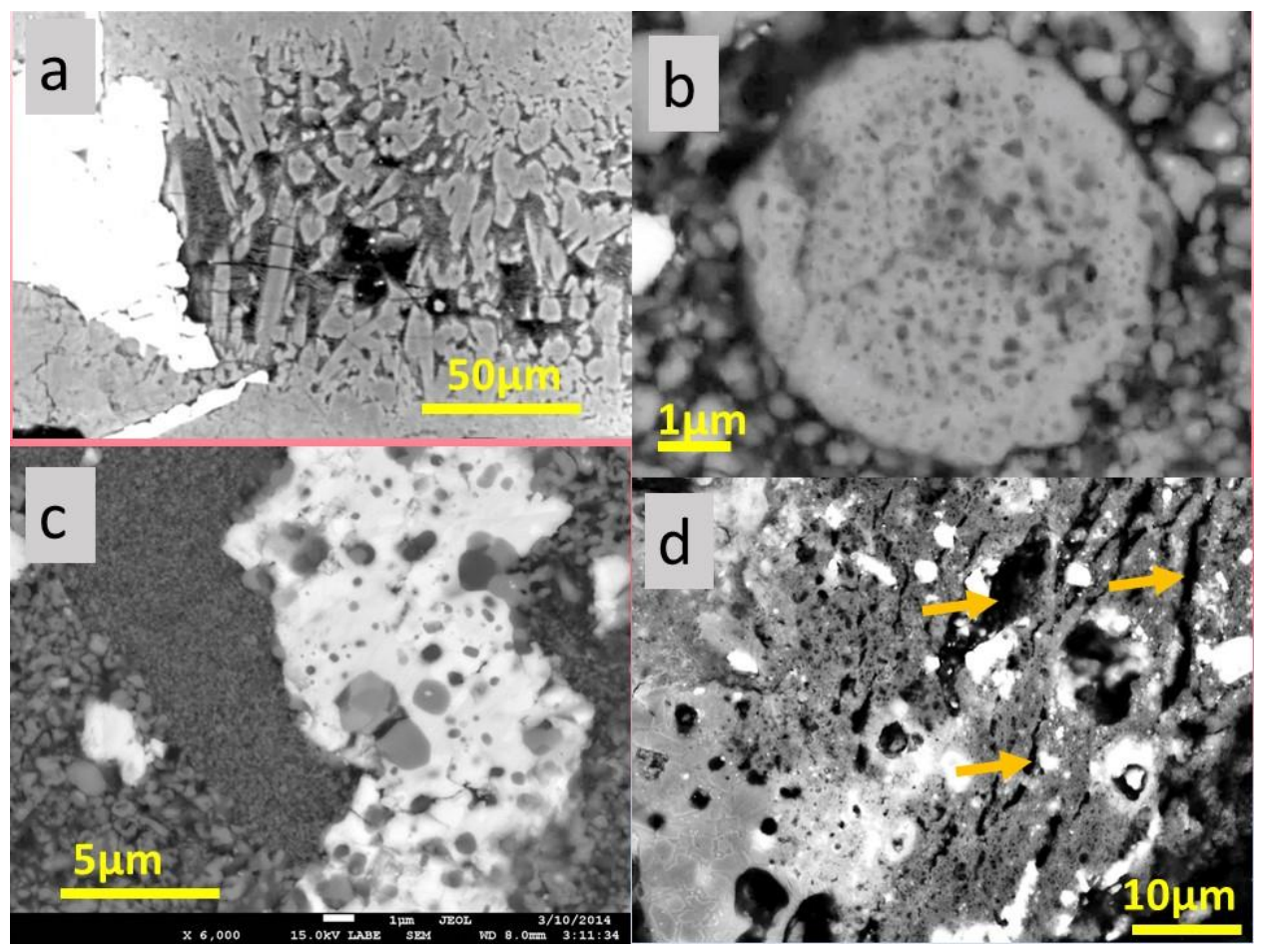

Figure 2.4. Shock melt textures in carbonaceous chondrites. (a) Aqueously-altered shock melt vein in the Kaidun meteorite CM1 lithology. Grey elongate crystals are skeletal, chemicallyzoned olivine that has been altered to serpentine and saponite. (b) (b) Glassy, vesicular impact melt spherule in the Orgueil CI1 chondrite. (c) Troilite (white) body in the Jbilet Winselwan CM chondrite that has been impact melted, and had matrix silicates (gray) forced into it before recrystallization. (d) Agglutinate in the Orgueil CI1 chondrite. Olivine crystals set in glass are at the lower left. Dehydrated phyllosilicates are at the upper right. Dessication cracks in the phyllosilicate are arrowed.

It is often thought that hydrous asteroids would generally disrupt, and not experience or preserve significant evidence of impact melting. However, we find that even the water-rich CI and $\mathrm{CM}$ chondrites contain evidence of impact shock. To see this one must look carefully at the regolith breccias, and see past the post-shock aqueous alteration, which has generally obscured mineral textures. We suggest that these materials will be present in significant quantity on the surfaces of C-class asteroids, where they can be explored by spacecraft such as Hayabusa2 and OSIRIS-Rex.

Several carbonaceous chondrite groups have preserved features from low-temperature aqueous alteration - most remarkably the CI, CM, and CR chondrites, which are henceforth collectively termed the hydrated carbonaceous chondrites. At the scale of meteorite-specimen bulk chemical composition, aqueous alteration of CI and CM carbonaceous chondrites is isochemical for all major rock-forming elements and all other non-volatile elements (DuFresne 
and Anders, 1962; Clayton and Mayeda, 1999; Young et al., 2003; Brearley, 2003, 2006; Rubin et al., 2007; Bland et al., 2009). Aqueous alteration reactions redistribute non-volatile elements between reactant and product host phases over sub-mm scales (Velbel et al., 2012, 2015), but the bulk abundances of rock-forming elements are identical among hydrated carbonaceous chondrite groups over the entire observed range of aqueous alteration (Brearley, 2003, 2006). Fine-grained and coarse-grained constituents of carbonaceous chondrites are all subjected to the same aqueous alteration conditions, but respond differently to aqueous alteration.

\subsubsection{Physical Properties, Chemical Composition, and Mineralogy of CM Chondrites and Processes Affecting these Meteorites}

\subsubsection{Physical Properties of CM chondrites}

Studies of the physical properites of carbonaceous chondrites remain limited (e.g., Corrigan et al., 1997; Miura et al., 2008; Rochette et al., 2008; Tsuchiyama et al., 2009; Macke et al., 2011; Britt et al., 2012; Jenniskens et al., 2012; Flynn et al. 2017). This is certainly the case for CM chondrites. Corrigan et al. (1997) measured the porosity and permeability of six CM2 chondrites (ALH 83100, ALH 85010, Bell, Murchison, Murray, and Nogoya) and one CM1 chondrite. They also measured the properities of EET 83226, which has since been reclassified as an ungrouped carbonaceous chondrite. Macke et al. (2011) measured the physical properties of $13 \mathrm{CM}$ chondrites (Banten, Cimarron, Cold Bokkeveld, Crecent, El-Quss Abu Said, Essebi, Mighei, Murchison, Murray, Nawapali, Nogoya, Pollen, Santa Cruz). To constrain intrameteorite variations, they measured 14 stones from Murchison and 12 from Murray. They found that all physical properties in these two meteorites varied $<10 \%$ from the mean value (Macke et al. 2011). Britt et al. (2012) measured the properties of the recent fall Sutter's Mill.

Porosity is one of the most important physical properties of meteorites. Not only does porosity have implications for the interaction between minerals and fluids, but Flynn et al. (2017) has argued that high porosities require higher kinetic energy for collisional disruption. Corrigan et al. (1997) measured the porosity of CM matrices to range between 2-13\% and the bulk porosity of Murchison to be $23 \%$. Macke et al. (2011) obtained a mean porosity of $24.7 \%$, ranging from $15.0 \%$ to $36.7 \%$, which is generally consistent with the values obtained by Corrigan et al. (1997). The porosity of Sutter's Mill also falls within this range, at $31.0 \% \pm 1.4 \%$ (Britt et al., 2012).

The average bulk and grain densities were $2.20 \mathrm{~g} / \mathrm{cm}^{3}$ (from 1.88-2.47 g/cm ${ }^{3}$ ) and 2.92 $\mathrm{g} / \mathrm{cm}^{3}\left(2.74-3.26 \mathrm{~g} / \mathrm{cm}^{3}\right.$ ) respectively (Macke et al., 2011). For Sutter's Mill, both the bulk density $\left(2.31 \pm 0.04 \mathrm{~g} / \mathrm{cm}^{3}\right)$ and the grain density $\left(3.34 \pm 0.02 \mathrm{~g} / \mathrm{cm}^{3}\right)$ are either in the higher end or just above the values for other CM chondrites.

The magnetic susceptibilities of CM chondrites are highly variable from meteorite to meteorite, ranging approximately two orders of magnitude (Rochette et al. 2008; Macke et al. 2011). Rochette et al. (2008) analyzed $53 \mathrm{CM}$ chondrites including falls, cold and hot desert finds and obtained average of $\log \chi=3.90 \pm 0.43$ (in $10^{-9} \mathrm{~m}^{3} / \mathrm{kg}$; range 3.08-4.89). The average that Macke et al. (2011) obtained for 13 samples is consistent with that value, $\log \chi=3.93$ (in $10^{-9} \mathrm{~m}^{3} / \mathrm{kg}$ ). Macke et al. (2011) noted that this value is below CI values, but spanned a range from 3.30 to 4.77 (Macke et al. 2011). Sutter's Mill also falls within that range at $\log \chi=4.30 \pm$ 0.08 (Britt et al. 2012). These variations have been attributed to differences in the abundance of 
magnetic minerals among different CM chondrites (Rochette et al. 2008).

Other physical properties have only been measured for a handful of CM chondrites. Corrigan et al. (1997) measured the liquid permeability of three CM chondrites: Mighei (0.51-1.4 mdarcy), Murchison (0.27-1.81 mdarcy), and Nogoya (0.065-0.54 mdarcy). They noted that porosity and permeability are not correlated. Ibrahim (2012) determined the average compressional wave velocity for CM chondrites to be $2335 \pm 574 \mathrm{~m} / \mathrm{s}$ and the average shear wave velocities equal $1472 \pm 352 \mathrm{~m} / \mathrm{s}$. They found the porosity and velocities are anticorrelated. The compressible strength for Murchison is $50 \mathrm{MPa}$ (Miura et al., 2008) and for Sutter's Mill is $82 \mathrm{MPa}$ (Jenniskens et al., 2012). Finally, Tsuchiyama et al. (2009) measured the tensile strength of Murray $(8.8 \pm 4.8 \mathrm{MPa})$ and of Murchison $(2.0 \pm 1.5 \mathrm{MPa})$.

\subsubsection{Primary (Nebular) Characterisitcs of the CM Chondrites}

$\mathrm{CM}$ chondrites are sufficiently numerous and abundant among carbonaceous chondrite falls and finds that available samples encompass a well-studied range of degrees / extents of aqueous alteration and corresponding compositional variations of aqueous alteration (e.g., Velbel et al., 2015, Table 2.1; Table 2.3 herein). Furthermore, there are as many meteorites or more in each of the CM1, CM1/2, and CM1-2 groups than the total number of CI1s, and these CMs have aqueous alteration attributes on a continuum with more numerous and better studied CM2s (Table 2.3). Thus, CM chondrites are the only materials presently available for laboratory study that preserve a partial but broad (Table 2.3) range of aqueous alteration phenomena relevant to the surface boulders and unconsolidated regolith materials yet to be encountered by the Hayabusa2, OSIRIS-REx, and future missions.

Minerals and glass in CM carbonaceous chondrites occur in coarse constituents ("larger components" as identified by Zolensky and McSween (1988), including chondrules, large mineral fragments, and refractory-element-rich calcium aluminum inclusions, CAIs), matrix (fine-grained), and fine-grained rims on coarse constituents. Some larger components (e.g., chondrules, chondrule fragments) consist of combinations of several solid phases, including olivine, pyroxene, and glass (Figure 2.5a); others consist of only one solid phase (e.g., monocrystalline olivine fragments). Rim and matrix (Figure 2.5a) consist of assemblages of fine-grained minerals, many of which are varieties of the same minerals that occur in the coarser constituents. Comprehensive recent overviews of CM2 mineral assemblages and their textures are available (Dodd 1981; Brearley and Jones 1998; McSween 1999; Krot et al. 2003; Hutchison 2004; Weisberg et al. 2006).

Bland et al. (2004) performed quantitative XRD analysis of the Murchison CM2 chondrite (a meteorite with an intermediate degree of aqueous alteration) with this result: $58 \%$ cronstedtite, $25 \%$ serpentine, $11 \%$ olivine, $2 \%$ low-Ca pyroxene (reported as clinoenstatite), $2 \%$ pyrrhotite, $1 \%$ calcite. However, this technique could not determine the relative abundances of poorly-crystalline materials (tochilinite, glass) nor of organics, and thus the relative mineral abundances reported are high and incomplete. Howard and co-workers have applied quantitative XRD to a large number of CM chondrites (Howard et al., 2009, 2011, 2015a). In a study of five CM falls, Howard et al (2009) found that four exhibited narrow ranges in the combined modal volume (vol\%) of $\mathrm{Mg}$-serpentine (25-33\%) and Fe-cronstedtite (43-50\%), similar to the previous studies. Cold Bokkeveld is anomalous in containing more Mg-serpentine (49-59\%) than Fe-cronstedtite (19-27\%); including Cold Bokkeveld, the range in modal total phyllosilicate for all five CM falls examined is 73-79\% (average $=75 \%$ ). More recent results include amorphous materials (Howard et al., 2015b). Takir et al. (this 
volume) discuss quantitative XRD bulk composition analyses in detail, because they are very relevant to IR matches.

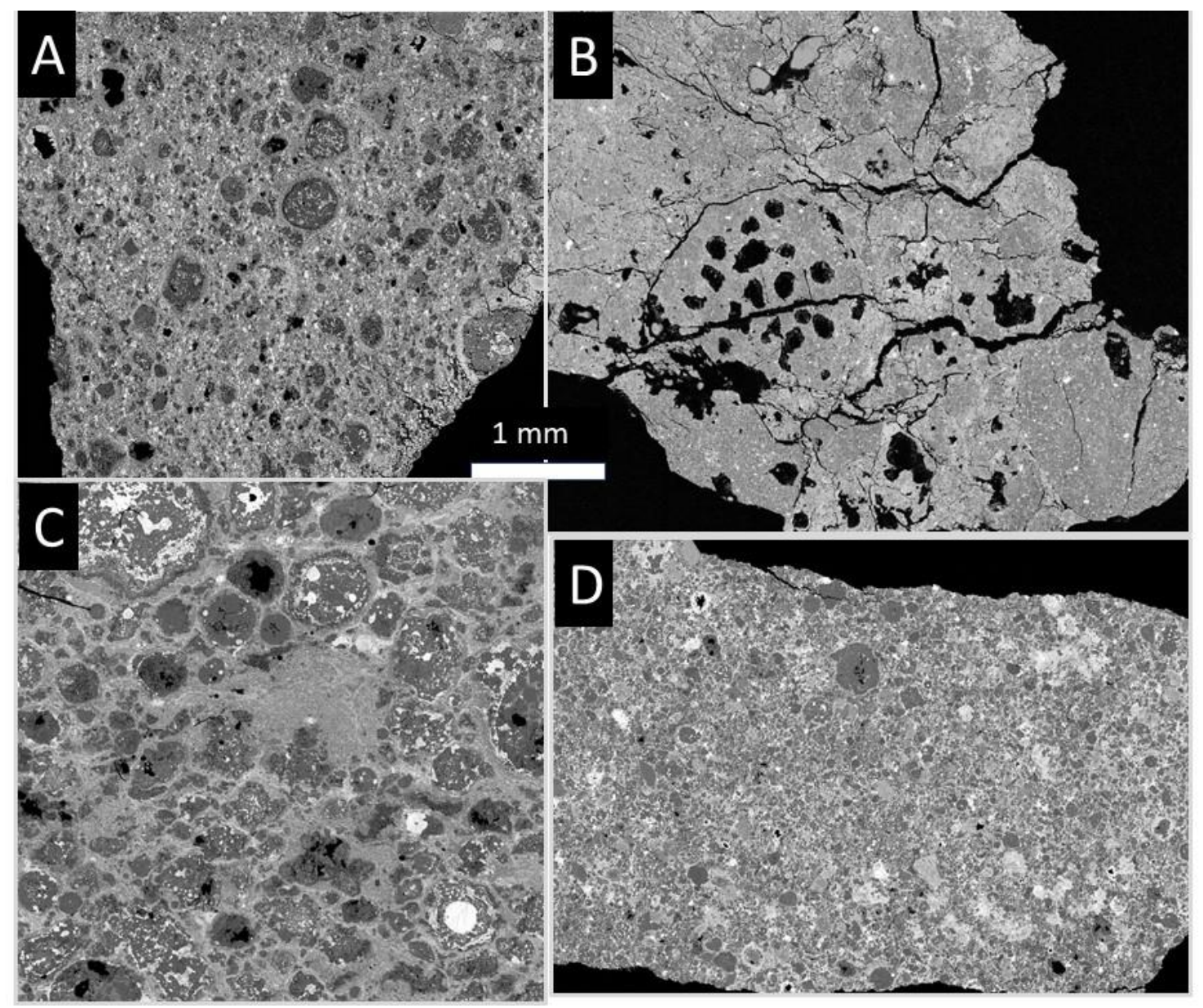

Figure 2.5: Comparison between overall textures of (A) a typical CM - Mighei, (B) a CI Orgueil, (C) a typical CV - NWA 10758, and (D) a typical CO - MIL 90019, all at the same scale. These are backscattered electron images. The CM and CI are dominated by fine-grained phyllosilicates, but the $\mathrm{CM}, \mathrm{CV}$ and $\mathrm{CO}$ contain chondrules, CAI and other larger components. The $\mathrm{CV}$ and $\mathrm{CO}$ are dominated by olivine, with significant pyroxene, plagioclase, troilite, and $\mathrm{Fe}-\mathrm{Ni}$ metal. Note the pronounced difference in chondrule sized between the $\mathrm{CM}, \mathrm{CV}$ and $\mathrm{CO}$. Most large black pores in Orgueil are sample preparation artifacts. The images of NWA 10758 and MIL 90019 are from D. Kent Ross.

\subsection{Chondrules in CM Chondrites:}

Chondrules, CAI and AOA in CM chondrites are typically smaller than in most other chondrutes, generally $<0.5 \mathrm{~mm}$ in greatest dimension, and have a fairly narrow size range (Hutchison, 2004). These materials are usually manelted by fine-grained material that is minerlogically essentially identical to fine-grained interchondrule matrix. In many, possibly most 
CMs the chondrules are uniformly flattened, imparting a foliation to the overall, probably due to impact deformation meteorite (Hanna et al., 2015; Hanna and Ketcham, 2018).

Chondrule genesis is not really well understood, and even less so for chondrules in carbonaceous chondrites since the bulk of work has been perfomed on chondrules in ordinary chondrites. Nevertheless, there are several studies and resulting theories concerning the formation of chondrules in CM chondrites (Cato and Fagan 2017). Due to their enrichment in volatile and moderately volatile elements, larger average size, and presence of relict grains, it has been suggested that type I chondrules formed as a product of evaporation and reduction that occured during heating of preexisting type II chondrules (Ruzicka et al., 2012). However, based on an examination of the Paris CM chondrite, Hewins et al (2015 argued that these differences result from the chondrule types having formed from separate nebular reservoirs (Hewins et al., 2012). Most recently, it has been suggested that type II chondrules in CMs have been derived directly from type I chondrules through oxidation processes based on zoning characteristics in relict olivine grains. This hypothesis was recently reinforced by evidence that heating events has the capability to form Mg-rich relicts similar to those observed in type II chondrules (Villeneuve et al., 2015), and petrographioc observation of the Jbilet Winselwan CM chondrite (Cato and Fagan, 2017).

Friend et al. (2016) reported that typical POP chondrules in CMs have olivine grains concentrated in the center and low-Ca pyroxene minerals at the rim, as is the case with typical chondrules in carbonaceous chondrites. Both, olivine and low Ca-pyroxene grains are unzoned and the transition from forsteritic olivine to $\mathrm{FeO}$-rich matrix is abrupt.

\subsection{Fine-Grained Materials in CM Chondrites}

Fine-grained matrix, chondrule mesostasis, and chondrule chondrule rims in unmetamorphosed CM chondrites are essentially mineralogically identical, although there are a few reported exceptions. For example, one chondrule rim in Murray has small amounts of saponite (Zolensky et al., 1993). The bulk of the fine-grained material is a range of serpentinegroup phases, based on microprobe analyses and lattice fringes observed by HRTEM.

Cronstedtite is most abundant in the less pervasively altered meteorites (first noted by Müller et al., 1979). Also present are more Mg-rich serpentine varieties including antigorite and chrysotile. Clinochlore has been been identified as a less abundant phase. Saponite is rarely observed in CM chondrites, in marked contract to CI and CR chondrites (Zolensky et al., 1993).

A phase almost unique in astromaterials to CM chondrites is the sulfide-hydroxide tochilinite (Mackinnon and Zolensky, 1984). Tochilinite is present in alll umetamorphosed CM chondrites. There have been many characterizations of the secondary minerals serpentine, tochilinite, and intergrowths of the two. The serpentines exhibit spongy, flaky, platy, and cylindrical morphologies and maximum grain sizes of approximately $10 \mu \mathrm{m}$. Tochilinite has similar morphologies but has a maximum grain size approximately one order of magnitude smaller. Tochilinite is almost always intergrow with serpentine phases, sometimes in a coherent intergrowth with a $1.7 \mathrm{~nm}$ lattice repeat. The intergrowths of tochilinite and serpentine have grain sizes like those of the former phase but appear to be restricted to platy morphologies. Tochilinite is expecially abundant in the Maribo CM chondrite (Haack et al., 2012).

It is reasonable to posit that the amount of serpentine in a chondrite could be related to the degree of aqueous alteration it experienced (McSween, 1979a). Tomeoka and Buseck (1985) first proposed that serpentine-like minerals in CM chondrites become progressively enriched in 
$\mathrm{Mg}$ (and depleted in $\mathrm{Fe}$ ) as aqueous alteration proceeds. Although the compositional distribution of serpentine analyses for most CM chondrites is considerable, certain compositions are prevalent in some cases. Serpentine analyses in some CMs reveal not two serpentine endmembers (commonly called cronstedtite and antigorite/chrysotile by chondrite workers) but rather a complete range of compositions (Zolensky et al., 1993). Serpentine family minerals volumetrically dominates matrix and chondrule rims in unmetamirphosed CM chondrites, as first noted by McSween (1979a,b) and Barber (1981).

Zolensky et al. (1993) reviewed the literature concerning the frequently observed "17 phase" $(1.7 \mathrm{~nm})$, proposing that it is a coherent intergrowth of tochilinite and serpentine. We have verified this hypothesis through the characterization of unusually coarse-grained crystals of this phase located among Mighei and Nogoya matrix. As both tochilinite and serpentine have considerable compositional ranges (Zolensky and Mackinnon, 1986; Zolensky, 1987), the same is expected for tochilinite-serpentine. The ratio of $\mathrm{Si} / \mathrm{S}$ would be set by the alternating nature of the tochilinite and serpentine layers and the number of fundamental units of each within the larger, incommensurate tochilinite-serpentine unit cell. However, even this ratio will be variable due to possible substitution of $\mathrm{A} 1$ for $\mathrm{Si}$ in the tetrahedral layers of serpentine, and $\mathrm{A} 1$ for $\mathrm{Mg}$ or $\mathrm{Fe}$ in octahedral, brucite-like layers of tochilinite. Additional complexity is introduced by the presence of additional stacking sequences of tochilinite and serpentine. The coherently interstratified tochilinite-serpentine-serpentine phase is observed in scattered, diminutive $(<100$ $\mathrm{nm}$ ) flaky and platy crystals in CM matrix and rim material. When tochilinite is present among platy serpentine (the coarsest morphology), it is coherently intergrown as tochilinite-serpentine or a higher-level intergrowth variety (e.g., tochilinite-serpentine-serpentine). If serpentine is recrystallizing and coarsening with ilicreasing alteration, then this observation may imply that tochiliniteserpentine is more stable than simple tochilinite as alteration proceeds.

\section{Carbonates in CM Chondrites}

All unmetamorphosed CMs contain calcite and minor dolomite. The CM calcites contain small amounts of $\mathrm{MgCO} 3$ and $\mathrm{MnCO} 3$, and FeCO3 (deLeuw et al., 2010). At temperatures 250 $\mathrm{C}$, the relative solubility for $\mathrm{FeCO} 3$ in calcite is higher than the solubilities of $\mathrm{MnCO} 3$ and $\mathrm{MgCO} 3$. The same appears to be true at lower temperatures (e.g., Be'ne'zeth et al. 2009). Accordonmg to deLeuw et al. (2010) the average CM calcite composition is $98.5 \mathrm{~mol} \% \mathrm{CaCO} 3,0.27 \mathrm{~mol} \%$ $\mathrm{MgCO} 3,0.90 \mathrm{~mol} \% \mathrm{FeCO} 3$, and $0.27 \mathrm{~mol} \% \mathrm{MnCO} 3$. The average $\mathrm{CaCO} 3$ content in the measured $\mathrm{CM}$ dolomite grains is $52.1 \mathrm{~mol} \%$; the average $\mathrm{MgCO} 3$ content is $38.8 \mathrm{~mol} \%$. The remaining 9 mol\% consists of approximately equal concentrations of $\mathrm{FeCO} 3$ and $\mathrm{MnCO} 3$. The results of this study indicate that carbonate formation in $\mathrm{CM}$ chondrites differs from that in CI chondrites and is more complex than generally thought, involving (1) carbonates formed on the parent asteroid in an aqueous environment that gradually changed in composition, (2) at some stage, $\mathrm{Ca}$ and $\mathrm{Mg}$ activities in the environment were high enough to form metastable dolomite, and (3) dolomites disappeared in the most aqueously altered CM chondrites.

\subsection{Refractory Inclusions in CM Chondrites CAIs in CM Chondrites}

CAIs are the earliest dated solar system solids: $\sim 4567.3 \mathrm{Ma}$ which is usually taken to be the age of the solar system. They have variously witnessed evaporation, condensation, aggregation, irradiation, and melting processes in a gas of solar composition (reduced \& ${ }^{16} \mathrm{O}$-rich) 
in region(s) with ambient $\mathrm{T}>1400 \mathrm{~K}$. In many cases they have experienced these processes repeatedly. The duration of CAI formation is not known, but it appears to overlap the earliest chondrule formation (Connelly et al., 2012). CAI are not especially abundant in CM chondrites, and are usually small and nodular, spinel-cored objects with pyroxene- and serpentine-rich rims (MacPherson, 2014). Thus there have been relative few studies dedicated to their study. In one notable series of studies (Fahey et al. (1987), Hinton et al. (1988), Ireland (1988, 1990); Ireland et al., 1988) correlated isotopic-petrologic studies of CM hibonite-rich inclusions are among the most detailed studies of large numbers of CAIs ever conducted. They demonstrated a huge diversity of trace element and isotopic patterns among petrographically-similar CAI, and showed that a significant fraction of hibonites in CM CAIs contain large nuclear (nonradiogenic) isotope anomalies coupled with lack of evidence for extinct ${ }^{26} \mathrm{Al}$. The isotopic diversity of these CAI testify to the chemical heterogeneity of the solar nebula (MacPherson, 2014) populations of grains, mostly formed directly as nebular condensates or derived from same, are powerful testimony to the isotopic heterogeneity of the solar nebula on a grain-tograin scale.

CAIs in the CMs are small, generally well below $1 \mathrm{~mm}$ in size. (MacPherson and Davis, 1994). These CAIs are spinel-pyroxene inclusions, with centers that are rich in spinel, with or without accessory hibonite and perovskite, and Wark-Lovering rims consisting mainly of aluminous diopside and iron-rich phyllosilicate. Hibonite-rich CAIs in CM chondrites are among the most-studied of all CAIs, because hibonite was expected to be one of the earliest solar nebula. CM CAIs are notable for the paucity of melilite. Melilite is only a minor accessory in CM CAI, and only in a very few of these (MacPherson and Davis, 1994). The aqueous alteration that has pervasively changed precuror CM minerals has severely changed the CAI as well. All CM CAI have rims of serpentine or cronstedtite, and sometimes these pohases are inside the CAI. A notable example is in the Maribo CM chontite where carbonates and tochilinite rim all CAI (Haack et al. 2012).

\section{AOA in CM Chondrites}

As in the other chondrite types, AOA in CM chondrites consist mostly of olivine, generally forsteritic, and usually have highly irregular shapes that suggest they are unmelted aggregates (Grossman and Steele, 1976). Many AOAs contain small nodules of refractory, CAI type minerals including spinel, calcic pyroxene, anorthite, perovskite, and more rarely melilite (Komatsu et al., 2001). The forsterite in AOAs is ${ }^{16} \mathrm{O}$ rich, suggesting that they formed in the same or identical oxygen isotopic reservoir as did the primary phases in CAIs (Krot et al., 2002).

\subsubsection{Mineral relations and petrography of aqueous alteration in CM2 chondrites}

Phyllosilicate-rich fine-grained rim and matrices constitute 47-85\% of CM chondrites and consist predominantly of serpentine-group minerals (McSween, 1979a; Hewins et al., 2014). CM chondrites were originally all assigned to petrologic type 2 (see discussion of Van Schmus and Wood, 1967 above). Subsequent research suggested that petrologic type 3 is the most primitive (least evolved in terms of either thermal metamorphism, understood to increase petrologic type, or aqueous alteration, understood to decrease petrologic type). Research on CM aqueous alteration reveals a range of degrees of aqueous alteration within CMs (McSween and Richardson, 1977; McSween, 1979a,b, 1987; Zolensky et al., 1993, 1997; Browning et al., 1996; Rubin et al., 2007; Chizmadia and Brearley, 2008; Howard et al., 2009, 2011, 2015; Takir et al., 2013; Alexander et al., 2013Garenne et al., 2014; Lee et al., 2014; Lindgren and Lee, 2015; 
Pignatelli et al., 2016; Lee and Lindgren, 2016). The community is presently in the midst of a paradigm shift, during which agreement has not yet been reached as to whether the range of CM alteration ought to be treated as ranging between petrologic types $2-3$ (Rubin et al., 2007), $2-1$ (Zolensky et al., 1997), or $3-1$.Regardless of the alteration scale used, CM matrix is initially Fe-rich $[\mathrm{Mg} /(\mathrm{Fe}+\mathrm{Mg}) \mathrm{wt} \% \approx 0.2]$ (e.g., Zolensky et al., 1993) and becomes progressively more $\mathrm{Mg}$-rich $[\mathrm{Mg} /(\mathrm{Fe}+\mathrm{Mg}) \mathrm{wt} \% \approx 0.8]$ in meteorites that show independent evidence of more extensive aqueous alteration (McSween and Richardson, 1977; McSween, 1979a,b, 1987; Zolensky et al., 1993, 1997; Browning et al., 1996; Rubin et al., 2007; Chizmadia and Brearley, 2008; Howard et al., 2009, 2011; Velbel and Palmer, 2011; Velbel et al., 2012, 2015; Takir et al., 2013; see review by Brearley, 2006).

Quantitative measures of the extent of aqueous alteration in comparative, multi-meteorite studies of CM chondrites have been based on data acquired by optical petrography (e.g., Browning et al. 1996), electron probe microanalyses (EPMA) of fine-grained matrix and rim materials (e.g., Zolensky et al. 1993; Browning et al. 1996) including "poorly characterized phase" (e.g., Rubin et al. 2007), transmission electron microscopy (Chizmadia and Brearley 2008), and quantitative determination of mineral abundances by X-ray diffraction (XRD) (Howard et al. 2009, 2011, 2015a). Broad compositional trends are recognized in fine-grained CM (carbonaceous chondrite, Mighei-type) chondrite alteration minerals (e.g., matrix, rims) and appear to be related to trends in degree of aqueous alteration within CMs of petrologic type 2 (Zolensky et al. 1993, 1997; Browning et al. 1996; Rubin et al. 2007; Chizmadia and Brearley 2008; Howard et al. 2009, 2011, 2015a), and from petrologic type 2 to type 1 in CM chondrites (Zolensky et al. 1997; Alexander et al., 2013; Howard et al. 2015a). However, different alteration metrics can yield varying results for the same meteorites, and differences in the suites of CM chondrites examined by different studies can create the appearance of broader or narrower ranges, and continuous or discontinuous variations, in degree of alteration (Velbel and Palmer 2011).

Zolensky et al. (1993) suggested that during early aqueous alteration, aqueous solutions in $\mathrm{CM}$ chondrites were dominated by Fe relative to $\mathrm{Mg}$, because of the rapid water-rock reactions with reactive $\mathrm{Fe}$ host phases. $\mathrm{CM}$ chondrites that experienced more advanced alteration were altered by solutions progressively enriched in dissolved $\mathrm{Mg}$ released by slower-reacting and thus still persistent in later solutions being richer in $\mathrm{Mg}$ relative to Fe. Solute evolution influenced by differential water-rock reaction kinetics of primary Fe- versus $\mathrm{Mg}$ - host phases controlled the compositions of fine-grained serpentine with progressive aqueous alteration of fine-grained rims and matrix in CM chondrites (Velbel et al., 2012, 2015). Such progressive alteration is expressed in a number of measurable and quantifiable parameters of CM chondrites (Table 2.3).

2.4.2.4. Progressive alteration of CMs and a Conceptual Model. Recent work expanded the conceptual model of aqueous alteration of chondrule phases in CM chondrites proposed by Hanowski and Brearley (2001). The original model proposed a four-stage sequence, relating the textures and compositions of phyllosilicates replacing coarse CM2 olivine, the extent and degree of olivine alteration to serpentine, and the chemical evolution of the co-existing aqueous solutions, culminating in the observed properties of ALH 81002 (Hanowski and Brearley, 2001). Following on their four-stage model, Velbel et al. (2012, 2015) added observations and inferences from Nogoya and QUE 93005, adding fifth and sixth stages. Table 2.3 lists all the CM2 chondrites studied by Velbel et al. (2015; their Table 1) and several CMs showing even higher degrees of aqueous alteration that have been classified by at least one of the previously 
proposed CM alteration scales (Zolensky et al., 1993, 1997; Browning et al., 1996; Hanowski and Brearley, 2001; Rubin et al., 2007; Howard et al., 2009, 2011, 2015). The following discussion (modified and updated from Velbel et al., 2012, 2015) explores mineral assemblages at different alteration stages in the context of the expanded conceptual model.

The model presented here assumes well-mixed starting materials, homogenous over spatial scales of centimeters to tens of centimeters, and consisting of anhydrous silicates, water ice, and other materials (possibly including previously hydrated silicates). The assemblage of primary minerals underwent closed-system (isochemical; DuFresne and Anders, 1962; Clayton and Mayeda, 1984, 1999; Young et al., 2003; Brearley, 2003, 2006; Rubin et al., 2007; melting of the local initial ice inventory (Clayton and Mayeda, 1984, 1999; Cohen and Coker, 2000; Rosenberg et al., 2001). Secondary phyllosilicates are emphasized, because they are the most abundant secondary phases.

Solute redistribution between primary and secondary minerals controlled the formation of secondary minerals. Two interconnected factors regulated the compositions of these secondary minerals: (1) primary mineral reactions rates and depletion and (2) composition of evolving aqueous solutes. The first factor, the compositions of fine-grained, Fe-rich primary mineral reactants dominated the compositions of secondary minerals during early-stage aqueous alteration of CM chondrites. Dissolved Fe dominated early aqueous solutions, released from fastreacting Fe-rich metal, sulfides, and Fe-rich primary anhydrous silicates. Fe-rich fines formed by alteration early in solute evolution. With continued alteration, Fe-rich reactants were progressively consumed and coarser, slower-reacting, Mg-rich reactant crystals (e.g., olivine and pyroxene in chondrules) continued to react with transient pore fluids. Consequently, at more advanced stages of alteration, the influence of the activity of solutes in aqueous media on the composition of secondary phases grew more preponderant (Velbel et al., 2012, 2015). More Mgrich, nearly homogenous, ferroan serpentine (serpentine-greenalite solid-solution) partially replaced olivines of diverse compositions during commonly incomplete and arrested alteration of coarse chondrule-hosted olivine. The excess $\mathrm{Mg}$ and Si exported from serpentinized partial pseudomorphs after Mg-rich olivine and pyroxene silicate phenocrysts dominated the more extensively evolved aqueous solutions, and were available to be incorporated into other phases spatially separate (outside the pre-replacement boundaries of; at least microns, possibly >mm$\mathrm{cm}$ or more away) from the chondrule reactants, including matrix $\mathrm{Mg}$-rich serpentine. $\mathrm{Mg}$-rich ferroan serpentines of similar compositions in fine-grained rims, matrix, and chondrules likely formed concurrently. Phyllosilicates are Fe-rich (e.g., cronstedtite) in weakly altered matrix and fine-grained rims and, based on EPMA estimates, contain both $\mathrm{Fe}$ (II) and Fe(III). The dominance of $\mathrm{Fe}$ (II) in late-stage serpentine-greenalite (relative to, cronstedtite) indicates that aqueous solutions became more reducing with reaction progress.

Stage 1 (CM2.5, 2.4/5): Anhydrous primary phases including mesostasis, sulfides, and metal control the composition of the local altering fluid. Mesostasis is usually the first chondrule phase to alter to phyllosilicates (Fuchs et al., 1973) in porphyritic olivine chondrules in which olivine is unaltered (Hanowski and Brearley, 2001, their Stage 1). Phyllosilicates formed by alteration of chondrule mesostasis are initially Fe-rich (e.g., Hanowski and Brearley, 2001) because of the abundant Fe made available to the aqueous solution by ongoing destruction of the mesostasis itself, and rapidly-reacting Fe-rich minerals (e.g., metal, sulfides; Palmer and Lauretta, 2011) outside the glass.

Stage 2: Selective alteration of reactive Fe-host minerals controls the composition of the 
local altering fluid. At the earliest stage of silicate phenocryst alteration (after alteration of mesostasis), rapid alteration of fine-grained metal and sulfides; Palmer and Lauretta, 2011) and fine-grained ferroan olivine (Howard et al., 2009, 2011) operated in hydraulically restricted finegrained rim and matrix (Bland et al., 2009) and created transient microenvironments in which each reactant mineral grain and its immediate neighbors controlled the activity of solutes that could be incorporated into secondary serpentine. The minerals available at the earliest stages of aqueous alteration were fine-grained (Brearley 2003, 2006), and rich in Fe (e.g., Fe-rich metal, sulfides, and Fe-rich primary silicates). Both the Fe-rich compositions and fine grain size (with large surfacea are to volume ratios) resulted in high kinetic reactivities (Brearley 2003, 2006). Consequently, Fe dominated over Mg; and early-formed, matrix serpentine was Fe-rich (McSween and Richardson, 1977; McSween, 1979b, 1987; Zolensky et al., 1993; Howard et al., 2009, 2011). Local primary-mineral control of product chemistry in initially diverse microenvironments gave rise to appreciable spatial heterogeneity in $\mathrm{Fe} / \mathrm{Mg}$ abundances in different matrix regions within individual CM2s (Bland et al., 2009; Howard et al., 2009, 2011). For example, while means and modes of matrix $\mathrm{Fe} /(\mathrm{Fe}+\mathrm{Mg})$ ratios vary systematically from weakly- to extensively-altered CM2s, wide ranges exist within the phyllosilicate-dominated matrix in each individual CM2 chondrite (e.g., Zolensky et al., 1993).

Stages 3 and 4: Progressive differential depletion of reactive minerals controls the composition of the altering fluid. As alteration proceeded, fine-grained reactive Fe-host minerals were largely consumed. With continued reaction of the remaining coarser and / or more magnesian primary minerals, $\mathrm{Mg}$ came to dominate over Fe in the aqueous solution (Hanowski and Brearley, 2001). Chondrule-hosted silicates were still only partially replaced at this more advanced stage of Mg-dominated solutes by virtue of their much larger grain size than matrix and rim minerals. Consequently, the composition of the serpentine replacing large chondrule silicates is more magnesian than the early-formed matrix phyllosilicates.

Stages 5 (CM2.2) and 6 (CM2.1): Coarse minimally reactive primary minerals control the composition of the altering fluid. Partial replacement of forsteritic olivine along with more fayalitic olivine in Nogoya (CM2.2, of Rubin et al., 2007; Stage 5, Velbel et al., 2012) indicates a more advanced degree of aqueous alteration than was recognized at the time of Hanowski and Brearley's (2001) study of ALH 81002 (Stage 4). QUE 93005 (CM2.1) has (Table 4) more phyllosilicate and significantly lower abundances of anhydrous silicates (Howard et al., 2011) and was recently assigned to Stage 6 (Velbel et al., 2015). At least small quantities of chondrulehosted olivine and pyroxene remnants are observed in Stage 4-6 CM chondrites (altered to CM 2.3 - 2.1; phyllosilicate/hydrous ratio 3.96 - 6.57; Table 2.3). The persistence of compositionally diverse, partially-replaced, chondrule olivines in these extensively altered CM chondrites indicates that either (1) the aqueous alteration episodes were too short to allow complete replacement, or (2) one or more reactants (most likely water) were fully consumed before chondrule olivine phenocrysts were completely replaced (Velbel et al., 2012, 2015). These two explanations are not mutually exclusive.

Mineral assemblages and compositions present upon cessation of aqueous alteration at Stages 4 - 6. Long alteration timescales and/or stagnant or slow-moving fluids enabled diffusional homogenization of the aqueous solutions in the low-permeability chondrite matrix gradually homogenized the aqueous solutions over progressively larger spatial scales (Young et al., 2003; Bland et al., 2009). Consequently, during advanced stages of alteration, the composition of the serpentine replacing chondrule silicate phenocrysts is much more uniform in any given CM2 chondrite (e.g., Hanowski and Brearley, 2001; Velbel et al., 2012, 2015) than the wide range of 
compositions preserved in faster- and earlier-altered fine-grained matrix and rim materials (e.g., Zolensky et al., 1993).

It remains to be established whether the observed partial replacement textures and assemblages indicate local, individual, episodic, short-lived, aqueous alteration episodes such as exhalative or impact-induced hydrothermal liberation / mobilization of water, or compositionally distinct and locally incomplete events during a larger-scale longer-term alteration episode, such as passage of a replacement front associated with larger-scale hydrothermal circulation into the volume of the CM chondrite parent body eventually sampled by individual CM chondrites containing partial-replacement textures (Velbel et al., 2012).

Despite the large number of CM samples in our collections, the range of (hydrous) phyllosilicates to anhydrous silicates among existing samples is only a fraction of the possible range (Howard et al., 2009). CM samples have not yet been recovered and recognized that show complete replacement of primary minerals in CMs. The fact that such samples have not been reportd does not necesarily mean that they never formed. Neverthless, based on samples available, it appears that alteration was truncated (Velbel et al., 2012, 2015). As noted above, Cold Bokkeveld, the most altered of the five CM falls studied by Howard et al. (2009), is unique among the $\mathrm{CMs}$ they examined in continat more $\mathrm{Mg}$-serpentine than Fe-serpentine. If $\mathrm{CM}$ material exists that represents alteration beyond the presently known range, its silicates might consist of serpentine-group minerals as matrix with higher $[\mathrm{Mg} /(\mathrm{Fe}+\mathrm{Mg})]$ than other $\mathrm{CMs}$, and complete pseudomorphs of serpentine after coarse (e.g., chondrule phenocryst) olivine and pyroxene without any primary-silicate remnants. Three objects that approach the petrographic criteria just suggested - EET 83334, ALH 88045, and one lithology in the complex Kaidun breccia - were reported, characterized, and classified as CM1 by Zolensky et al.

(1993).Spectroscopic data from Dawn mission indicate that the surface material in C-type dwarf planet 1 Ceres is dominated by $\mathrm{Mg}$-serpentine, and thus records an extent of aqueous alteration beyond any present in meteorite collections (Ammanito et al., 2016; McSween et al, 2016). It remains to be established how the specific aqueous alteration phenomena summarized here, or phenomena extrapolated from them, are expressed for other primary minerals and in even more altered CM chondrites, and what other similar and / or related phenomena occur there.

\subsubsection{Regolith Processes Affecting CM Chondrites and CM Chondrite Hosts.}

Boundaries between clasts of different CM lithologies, or between a clast and surrounding matrix, are commonly sharp and distinct, although when clast lithologies different mainly in thir degree of alteration (e.g., Paris), clast boundaries can be "ill-defined" (Leroux et al., 2015). An example of a CM2 with many lithologies is shown in Figure 2.6. This meteorite, LON 94101, has at least 20 diferent documented CM2 litholohies (Lindgren et al., 2013).

Texture within clasts range from very similar to host materials, to very different. Boundaries in many occurrences can be resolved by optical petrography; more numerous and more subtle textural distinctions can be discerned using scanning electron microscopy. Clast outlines range from angular to rounded, often in the same specimen. Within-clast textures (e.g., chondrule to matrix ratios) vary widely. Distributions of relict primary phases and secondary phases vary widely, often in the same specimen. Particulary thorough and detailed work can distinguish CM lithologies from one another and from clasts of $\mathrm{CV}, \mathrm{CO}$, or ungrouped CC lithology (e.g., Zolensky and Ivanov, 2003, and MacPherson et al., 2009).

Extensive mixing of CM lithologies of varying degrees of aqueous alteration and other carbonaceous chondrite lithologies is known from clasts found in many meteorites. These 
include CM2 clasts in howardite-eucrite-diogenite (HED) group meteorites (Zolensky et al., 1996; Prettyman et al., 2012), and CM1-like lithologies in the unique carbonaceous chondrite breccia Tagish Lake (Brown et al., 2000). Zolensky and Ivanov (2003) and MacPherson et al. (2009) distinguished clasts of CV3, CM2, CR, CI, several different CM1 and ungrouped C2, and C1 lithologies in the polymict chondrite breccia Kaidun. If the lithologies are very distinct (e.g., carbonaceous chondrite clasts in HEDs, Kaidun), juxtaposition of different lithologies is easily observed among small clasts in a single stone. If the range of lithologies is narrower (e.g., CM2.1, CM2.0, and thermally metamorphosed C-chondrite lithologies in single stones of Sutters Mill), only detailed textural and compositional characterization of small-scale areas and volumes reveals the heterogeneities.

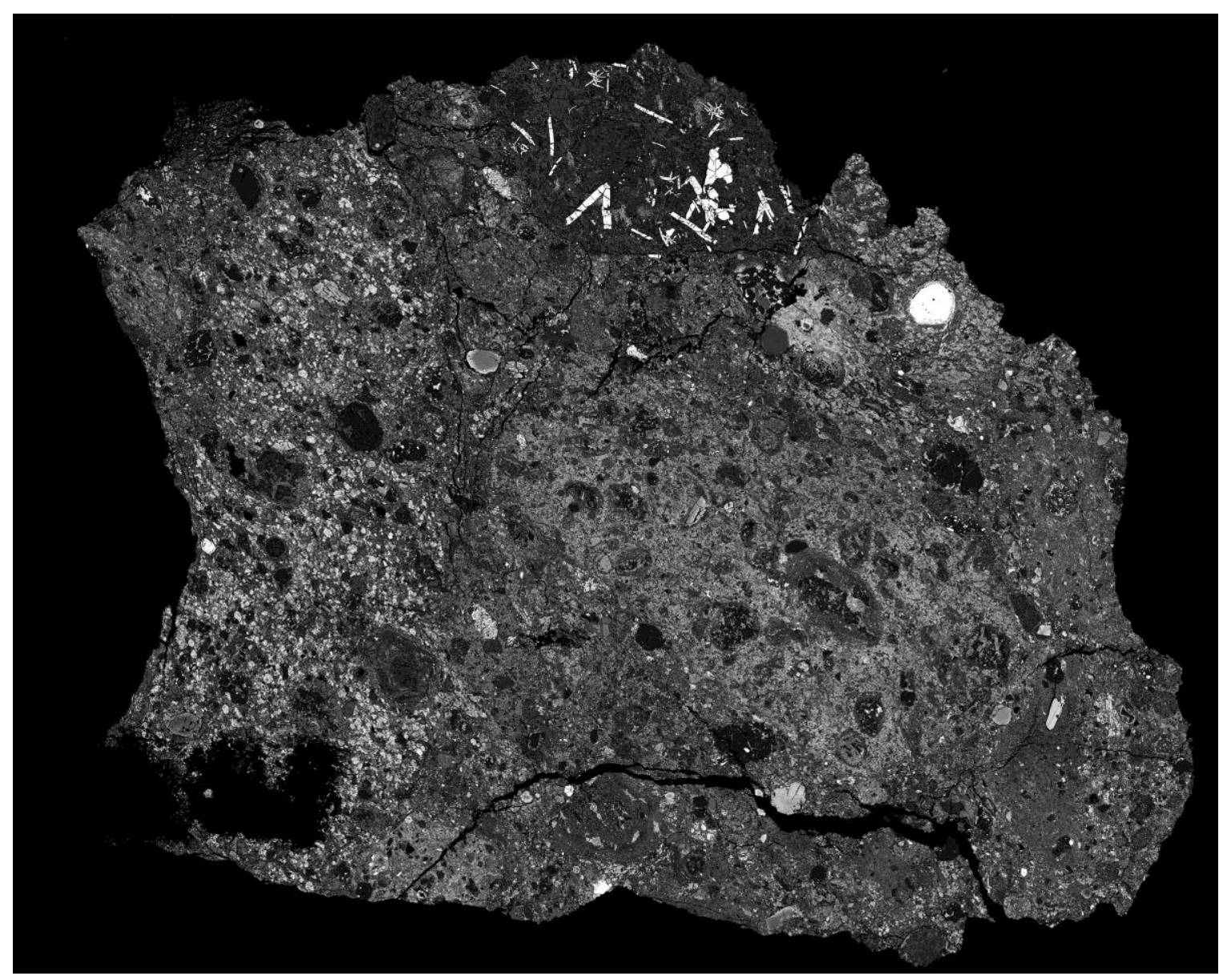

Figure 2.6. BSE image of LON 94101 showing numerous, quite dispatate lithologies due mainly to differences in chondrule rims, minral grain sizes and degree of breccciation. Most of the meteorite consists of phyllosilicates, Fe-Ni sulfides are white. View measures $3 \mathrm{~cm}$ across.

In addition to CM fragments found in other meteorite groups, Bischoff et al. (2006) found that all CM (as well as CI and CR) chondrites that they analyzed contained solar-wind-implanted gases, indicating that the hydrated carbonaceous chondrites are regolith breccias. Consequently, regolith brecciation must be considered in interpreting variations of different attributes within and among CM chondrites. In CM chondrite breccias, the range of lithologies can be narrow; for 
example, in Paris the only lithologic variations among clasts are between fresh or less altered metal-rich areas and moderately altered metal-poor zones CM lithologies (Hewins et al., 2014; Leroux et al., 2015; Rubin, 2015; Pignatelli et al., 2016, 2017; Stephant et al., 2017); and CM2.1, CM2.0, and thermally metamorphosed CC lithologies occur in single stones of the CM2 fall Sutter's Mill (Jenniskens et al., 2012).

CM clasts generally show different degrees of aqueous alteration compared to their host CM chondrites. For example, Lindgren et al. (2013) suggested that alteration of clasts occurred prior to assembly and lithification of CM regolith breccia LON 94101. Metzler (1995) reported clasts of widely varying extent of aqueous alteration in a single thin-section of CM2 Nogoya.

Grains the same sizes as CM clasts are also likely in regoliths of carbonaceous asteroids. The disk-averaged thermal inertia of 101955 Bennu (OSIRIS-REx target) is sufficiently similar to that of 25143 Itokawa (Hayabusa target) to suggest that 101955 Bennu has a surface texture and rubble-pile character similar to 25143 Itokawa (Müller et al., 2012). The best spectroscopic match for 101955 Bennu and individual meteorites are: (1) a sample of CI1 Ivuna experimentally heated to $700^{\circ} \mathrm{C}$, (2) the anomalous CM Dhofar 225, and (3) a matrix-rich fraction of CM2 Mighei, all examined as powders of 100-200 $\mu \mathrm{m}$ grains (Clark et al., 2011).

Stardust's comet sample is the only returned sample of carbonaceous-chondrite material to date from a known specific small-body. Existing evidence suggests that material similar in mineral relations and grain size to Stardust grains and the clasts of CM chondrite regolith breccias constitute the regolith of B-type asteroid 101955 Bennu. Observations from carbonaceous chondrite breccias must therefore inform our expectations about the range and heterogeneity of aqueous alteration that OSIRIS-Rex will encountered in sub-centimeter samples. Grains returned from comet 81P/Wild 2 by the Stardust mission include thermally unaltered grains of carbonaceous chondrite material (Brownlee et al., 2006; Zolensky et al., 2006, 2008), which may be partially comminuted fines, dispersed into comet 81P/Wild 2's coma by venting from the comet's nucleus. Stardust quenched metal-sulfide droplets (e.g., Leroux et al., 2008; Velbel and Harvey, 2009) have size distributions identical with those of intact sulfides in interplanetary dust particles with carbonaceous-chondrite affinities (e.g., anomalous CC Acfer 094; Sekigawa and Keller, 2010; Sanders and Velbel, 2012). This suggests that the size distribution of the Stardust quenched metal-sulfide droplets is a palimpsest of the smallest sulfide particle sizes into which cometary regolith of CC affinity disaggregates.

Assessing the timing of volatile incorporation into carbonaceous chondrite solids and the effect of the volatiles and the timing of their incorporation on detectable attributes are necessary to anticipating the properties of the regolith. If a meteoroid was assembled from fragments with uniform volatile abundances (e.g., accreted homogenous or uniformly altered fragments), such uniformity would suggest that: (1) the altering medium must have been compositionally homogenous over a vast volume of the clasts' source region or (2) the aqueous alteration occurred after the clasts assembled into their present host breccia. Subsequent mixing of clasts with fragments from sources with different alteration indices would also be precluded. In contrast, the coexistence of clasts with different alteration indices in the same breccias would indicate that varying degrees of aqueous alteration existed in the clast source areas before final assembly, and any post-assembly aqueous alteration was too minor and / or localized to homogenize the material.

Textural relationships between clasts and hosts provide clues to the spatial scale of physical and chemical differentiation, impactor-target-ejecta-regolith mixing, and homogenization interactions, and possibly also of the spatial scales of former hydrologic systems 
on asteroidal parent bodies. For example, juxtapositions of carbonaceous-chondrite clasts with different degrees of aqueous alteration (e.g., Kaidun, Tagish Lake, Sutters Mill) suggest a variety of spatially distinct aqueous alteration settings that may in turn represent differential mineralchemical alteration of post-accretionary solids. These aqueous alteration settings were subsequently disrupted and mixing of clasts ensued upon impact and regolith gardening.

Takenouchi et al. (2013, 2014), Zolensky et al. (2015, 2017), and Gregory et al. (2015) investigated possible pre-atmospheric juxtaposition of known CM2 finds in a heterogeneous carbonaceous chondrite breccia / rubble pile asteroid / meteorite, using cosmic ray exposure (CRE) ages for CM2s. See Figure 2.7. Nishiizumi and Caffee (2009) identified specific CM2s constituting 200ka and 600ka launch-paired groups of CM2 chondrites, and a number of other CM2 chondrites not associated with either of these launch-paired groups or with one another. Petrographic and / or compositional heterogeneity among the launch-paired CM2s ejected simultaneously from the same parent body would indicate that, within the last million years as indicated by the CRE exposure age, all specific lithologies of the CM2s in such a CRE-age cluster were juxtaposed with one another in the asteroid disrupted at that time. This would establish specific kinds of heterogeneity of lithologies coexisting in the larger-than-stone-scale parent body less than one million years ago. Such juxtaposition is more recent and on a larger spatial scale than the lithologic variation within a single CM2 stone, even a brecciated one. For example, the variation in aqueous-alteration parameters within different samples from a single launch-paired group will give insight into the range of alteration experienced by different parts of a single CM2 parent body.

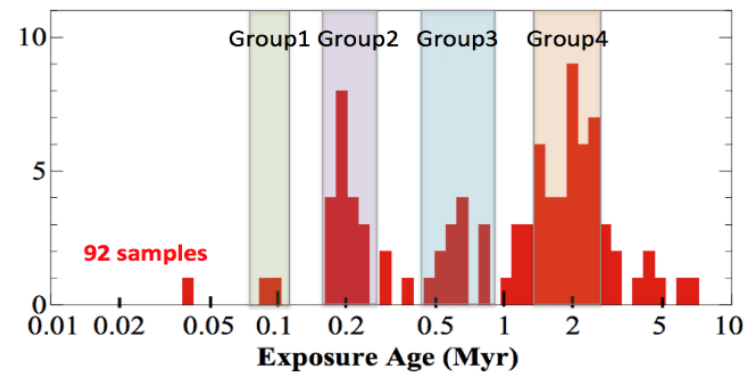

Figure 2.7. CM CRE ages, showing possible groupings, from Nishiizumi and Caffee (2012).

\subsubsection{Terrestrial Chemical and Mineralogical Weathering Processes Affecting the CM Chondrites}

Freshly fallen meteorites, recovered promptly after their witnessed fall, are referred to as 'falls.' Most meteorites available for scientific study are 'finds,' recovered after unwitnessed arrival and some exposure to the terrestrial surface environment, often over millennial or longer timescales. In order to interpret aqueous alteration phenomena in meteorites, scientifically informative pre-terrestrial (parent body) alteration must be distinguished from terrestrial alteration.

There is little published information on terrestrial weathering phenomena and products of CM chondrites. Unlike ordinary chondrite falls, that are sufficiently abundant to provide numerous samples minimally compromised by terrestrial weathering for comparison with 
mission data, many groups of carbonaceous chondrites consist of small numbers dominated by finds. Despite the abundance of finds among $\mathrm{C}$ chondrites, and unlike the case for OCs, very few dedicated studies of terrestrial weathering of carbonaceous chondrites have been published. Given the broad similarities between the mineral inventories of OCs and CCs, much that is known from numerous studies of terrestrial weathering of OCs (Velbel 2014 and primary references therein), transferability of many insights from OCs to CCs can be expected.

Eevaporite efflorescences comprising sulfates of the alkali and alkaline earth cations are widespread on CCs (e.g., Velbel and Losiak 2011; Losiak and Velbel 2011; Velbel and Palmer 2011). The cations and anions of these sulfates may be remobilized pre-terrestrial evaporites, or the sulfate anions are oxidation products of sulfides and the sulfate minerals' cations were leached from primary meteorite phases (Gounelle and Zolensky 2001; Losiak and Velbel 2011; Velbel and Palmer 2011). Such reactions are known both to proceed as post-recovery continuations of weathering initiated during pre-recovery weathering in Earth's natural environment, and in some instances even originate in curatorial and laboratory settings (Gounelle and Zolensky 2001; Losiak and Velbel 2011; Velbel and Palmer 2011). Carbonate- and sulfate-forming weathering in curatorial and laboratory processing environments has been documented for several carbonaceous chondrite groups. Reactive soluble species were remobilized and formed sulfates during curatorial storage of the CI carbonaceous chondrite Orgueil (Gounelle and Zolensky 2001), and redistribution of carbonate during sample storage has been documented in Vigarano (CV3) (Abreu and Brearley 2005).

The recently recognized redistribution of sulfur species in long-curated CI carbonaceous chondrite falls (Gounelle and Zolensky 2001) is of particular significance to C-chondrite sample return. Scientifically important properties of carbonaceous chondritic materials can be compromised by post-recovery terrestrial weathering reactions (hydrolysis and oxidation) during storage and processing in terrestrial curatorial and laboratory environments on time scales of decades (e.g., Gounelle and Zolensky, 2001). Highly soluble indigenous C-chondrite sulfates are easily leached when samples are rinsed with water at any stage of processing and / or preparation for analysis, providing a means of removing mobile elements temporarily stored in such soluble host minerals from the analyzed volume of the specimen (Velbel and Palmer 2011). If the sulfate and carbonate evaporites on carbonacoues chondrites are not redistributed indigenous sulfates and carbonates but are instead products oncoprating acid anions formed by terrestrial oxidation of, respectively, sulfide-hosted $\mathrm{S}$ (forming terrestrial sulfuric acid) and organic-molecule-hosted C (forming terrestrial carbonic acid), even brief exposure of returned carbonaceous samples to terrestrial moisture and oxidants can have significant deleterious influence on sample integrity. These sample-integrity vulnerabilities must be considered in planning for preservation of sample integrity during processing of newly recovered CM and CI chondrites and returned samples from sample-return missions to asteroids with carbonaceous-chondrite affinities.

\subsubsection{Implications of Secondary Process for Exploration of CM Parent Bodies.} Selection of sample sites on the CM- and CI-like target asteroids of the Hayabusa2, OSIRISREx, and future sample return missions will need to consider the overall degree of aqueous alteration of the regolith clast population in order to collect samples with the largest proportion of highly altered, phyllosilicate-rich clasts. CM chondrite aqueous alteration is essentially isochemical - bulk abundances of major and minor elements have small variations over small ranges throughout the known range of alteration (Brearley, 2003). Consequently, remote analysis of major or minor elements (e.g., by Alpha Particle (or Proton) X-ray Spectroscopy - APXS, or 
Laser Induced Breakdown Spectroscopy - LIBS) is not expected to detect compositional variations that indicate or proxy for abundances of volatiles that may influence geotechnical properties. Two of the best correlation relationships between measurable quantities and the overall degree or extent of aqueous alteration are H/Si ratios (Table 2.3) determined by elemental analysis (Van Schmus and Hayes, 1974) and the modal-abundance ratio of hydrous (phyllosilicate) minerals to anhydrous (primary silicate) minerals determined by quantitative $\mathrm{X}$ ray diffraction (Howard et al., 2011). These ratios range from $<3$ in the least altered CMs known, up to >9 in the most altered know; Howard et al., 2009, 2011). Neutron / gamma-ray spectroscopy is a remote (stand-off) method that can quantify H (Lim et al., 2017).

Another approach to quantifying aqueous alteration in CM parent bodies is identifying host minerals for volatiles directly (rather than by elemental abundances). Ratios of hydrous to anhydrous silicates can be determined by remote VIR spectroscopy (e.g., McAdam et al., 2015; Friedlander et al., 2016; Kaplan and Milliken, 2016).

\subsubsection{Physical Properties, Chemical Composition, and Mineralogy of CI Chondrites and Processes Affecting these Meteorites}

\subsubsection{Physical Properties of CI chondrites}

Some physical properties have been measured for Alais, Ivuna, Orgueil, Revelstoke, and Tonk (Corrigan et al. 1997; Consolmagno and Britt 1998; Rochette et al. 2008; Macke et al. 2011). The matrix porosities are $2 \%$ for Alais, $5 \%$ for Ivuna, and $4 \%$ for Orgueil (Corrigan et al. 1997). In contrast, Consolmagno and Britt (1998) reported the porosity of Orgueil to be $35 \%$. Macke et al (2011) argued that this large difference in porosity may have resulted from compression during sample preparation in the Corrigan et al. (1997) study. Consolmagno and Britt (1998) also obtained grain density $\left(2.43 \mathrm{~g} / \mathrm{cm}^{3}\right)$ and bulk density $\left(1.58 \mathrm{~g} / \mathrm{cm}^{3}\right)$ for Orgueil. Rochette et al. (2008) obtained average magnetization of $\log \chi=4.66 \pm 0.09$ (in $10^{-9} \mathrm{~m}^{3} / \mathrm{kg}$; range 4.58-4.78) for five CI chondrites. Finally, Tsuchiyama et al. (2009) measured the tensile strength of two CI chondrites, Ivuna $(0.7 \pm 0.2 \mathrm{MPa})$ and Orgueil $(2.8 \pm 1.9 \mathrm{MPa})$.

\subsubsection{Primary (Nebular) Characteristics of the CI Chondrites}

The CI chondrites have apparently inherited bulk compositions most similar to that of the solar photosphere (Anders and Grevesse, 1989), which belies their pervasively, aqueously altered character. Unlike all other chondrites, CI chondrites contain no chondrules, although they do include the alteration products of coarse-grained silicate materials such as CAI (Frank et al., 2017). The lack of CI2 or CI3 meteorites makes correctly understanding the protolith lithology a guessing game. Approximately half of the CI chodrites have been thermally metamorphosed.

\subsection{Relic Chondrules in CI Chondrites:}

Potential relict chondrules were reported in CI chondrites many years ago (Hyman and Rowe, 1983), but this report has never been properly verified. Thus at this time it is best to report that there are no unambiguous relict chondrules reported in any CI chondrite. 


\subsection{Fine-Grained Materials in CI Chondrites}

$\mathrm{CI}$ chondrites are dominated by fine-grained phases. In the half of $\mathrm{CI}$ chondrites that are not thermally metamorphosed this material is mainly an integrowth of serpentine- and saponitetype phyllosilicates with embedded sulfides such as pyrrhotite, pentlandite and cubanite, oxides such as magnetite and chromite, and iron oxyhydroxides such as ferrihydrite. This material is believed to have formed on or within the parent (Tomeoka et al., 1989b; Ikeda, 1992; Zolensky et al., 1993; Tonui et al., 2014). There are also present scattered residual grains of olivine, highand low-Ca pyroxene, and refractory phases. Fe-Ca-Mg carbonate grains are locally abundate. Magnetite takes the form of framboids, placquettes, and euhedral crystals. Bland et al. (2004) performed quantitative XRD analysis of the Orgueil CI chondrite with this result: $68 \%$ intergrown saponite and serpentine, $9 \%$ serpentine (alone), $7 \%$ magnetite, $5 \%$ sulfides, $5 \%$ ferrihydrite, $4 \% \mathrm{Fe}-\mathrm{Mg}$ carbonate, $1 \%$ olivine. However, this technique could not determine the relative abundances of poorly-crystalline materials including organics. Thus the relative mineral abundances reported are upper estimates. Organics are also present aong the matrix CI material, although its elucidation has barely begun (Pizzarello et al., 2005).

\subsection{Relic Refractory Inclusions in CI Chondrites:}

All CIs have experienced almost complete alteration from aqueous fluids within 5 million years of CAI formation, and as such, have had their primary mineralogy by fine-grained phyllosilicates, magnetite, carbonates, phosphates and sulfides. The only surviving indication of the original mineralogy of these rocks is the occasional, and extremely rare, isolated grains of severely corroded olivine and low-calcium pyroxene, and spinel. There has been no reported evidence of surviving primary components such as chondrules, amoeboid olivine aggregates or CAIs, which were probably once abundant. One relatively unaltered CAI has been decribed from the Ivuna CI chondrite, and its description of this CAI is entirely from Frank et al. (2017).

The CAI is roughly spherical, and is surrounded by the typical Ivuna phyllosilicate matrix. Therefore, it does not appear to be part of an unusual clast and there is no obvious evidence of brecciation. It lacks any Wark-Lovering Rim. Incipient aqueous alteration has occurred along the edge of the CAI, within the accretionary rim, and in veins that project from the rim inward towards the core. It is extremely fine-grained, with only a few grains exceeding 10 microns. The accretionary rim is host to a local high concentration of embayed crystals $>5$ microns of olivine ( $\left.\mathrm{Fa}_{2-17}\right)$, low-Ca pyroxene ( $\left.\mathrm{Fs}_{2-10}\right)$, pyrrhotite, and magnetite, all floating within phyllosilicates. EMPA analyses of the olivines in the rim compared to isolated olivine grains in the Ivuna phyllosilicate matrix show that their $\mathrm{Cr}$ and $\mathrm{Mn}$ contents as a function of their $\mathrm{FeO}$ content are indistinguishable. On the other hand, the CAI olivines are enriched in $\mathrm{Ca}$ with respect to the matrix olivines, but this could be explained as the result of Ca depletion that commonly occurs during aqueous alteration. Indeed, FE-SEM element maps show an obvious inverse correlation between $\mathrm{Ca}$ and $\mathrm{Fe}$ throughout the regions that have been altered. Therefore, the CAI may actually be close in size and shape to the pre-altered object and that, along with its accretionary rim, was largely spared by the usual CI1 total alteration. If this is true, then the olivine and pyroxene present within the accretionary rim may be remnants of a less altered CI (CI2?) precursor lithology. The oxygen isotopic composition $\mathrm{f}$ this CAI is consistent with that observed in the CAI in all other carbonaceous chondrites.

\subsubsection{Asteroidal Chemical and Mineralogical Processes Affecting the CI Chondrites}


The CI chondrites have seen a very wide range of chemical and physical processes, including condensation, accretion onto one or perhaps multiple generations of parent bodies, aqueous alteration, dehydration, impact processing, and thermal metamorphism (Tomeoka et al., 1989b; Tonui et al., 2014).

\subsection{Models of Aqueous Alteration Processes Affecting the CI Chondrites}

The CI chondrites were pervasively affected by multiple episodes of aqueous alteration under varying physical and chemical conditions (Endress and Bischoff, 1996). However, unlike the situation for the CM and CR chondrites, which have clearly defined alteration sequences (Howard et al., 2015), in terms of bulk phyllosilicate abundance there is no obvious difference in the extent of hydration recorded in the CI chondrites (King et al., 2015). This is consistent with their similar bulk $\mathrm{H}$ abundances (Alexander et al., 2012) and perhaps signals the cessation of fluid availability, truncating the process of hydration.

In the model of Tomeoka and Buseck (1988), aqueous alteration initially produced relatively coarse grained phyllosilicate clusters, along with magnetite, pyrhotite, pentlandite, cubanite, and carbonates. Further alteration then reduced the grain size of the phyllosilicates, and caused dissolution of the magnetite and sulfides that enabled formation of ferrihydrite. Orgueil, containing fine grained phyllosilicates and ferrihydrite, plus corroded magnetite and sulfide grains, is often considered the most altered CI chondrite (Tomeoka and Buseck, 1988; Bullock et al., 2005). Meanwhile, a lack of ferrihydrite suggests to some workers that Ivuna is the least altered. However, Zolensky et al. (1993) and Zolensky and Gounelle (2001) proposed that all ferrihydrite and the bulk of sulfates in chondrites has a purely terrestrial origin, which would eliminate the need for a late stage highly oxidizing process on the CI parent body. This proposal is supported by $\mathrm{O}$ isotope data for CI sulfates (Airieau et al., 2005).

\subsection{Thermal and Hydrothermal Metamorphism in CI Chondrites}

Half of the known CI chondrites have been heated (either by thermal metamorphism, solar heating, or impact heating), and in these phyllosilicates are replaced first by highlydisordered and defect-rich structures and finally by olivine, and carbonates are replaced by oxides (Tomeoka et al., 1989b; Tonui et al., 2014, Tomoki Nakamura, personal communication, 2016). Thermal metamorphism in these chondrites is apparent in their low contents of $\mathrm{H}_{2} \mathrm{O}, \mathrm{C}$ and the most thermally labile trace elements, partial dehydration of matrix phyllosilicates and abundance of thermally decomposed $\mathrm{Ca}-\mathrm{Mg}-\mathrm{Fe}-\mathrm{Mn}$ carbonates, which apparently resulted from heating of $\mathrm{Mg}-\mathrm{Fe}$ carbonate precursors.

Both coarse- and matrix- phyllosilicates in the metamorphosed CI chondrites Y-86029 and Y-82162 show partial dehydration of phyllosilicates evident in high analytical totals ( 87-98 wt\%). TEM analyses of these phyllosilicates in Y-86029 show highly disordered and defect structures with lattice fringes between $9 \AA$ and $13 \AA$, and localized areas with 4.7 to $4.8 \AA$, which represent mixtures of residual serpentine and neoformed olivine. Phyllosilicates in Y-82162 also show lattice fringes between $9 \AA$ and $12 \AA$ and flaky elongated grains with spacings of $\sim 9.5 \AA$, which Akai (1990a,b) interpreted as corresponding to dehydrated saponite. XRD analysis of Y86029 matrix shows general broadening of olivine typical of incomplete (fine-grained) recrystallization of olivine after thermal decomposition of phyllosilicates. Such minerals with highly-disordered and defect structures have been described in terrestrial samples (e.g. Brindley and Hayashi, 1965) as being formed from the transformation of serpentine- or saponite- type 
phyllosilicates (Akai, 1990a,b). Experimental data show that thermal transformation of saponite occurs at $900{ }^{\circ} \mathrm{C}$, while basal reflections of saponite before structural decomposition, have been reported to be $9.7 \AA$ at $500^{\circ} \mathrm{C}$ and $9.5 \AA$ at $600-750^{\circ} \mathrm{C}$ (Midgley and Gross, 1956; Akai, 1990a,b). These values also correspond to the interlayer spacing in talc. Transformation of serpentine to forsterite commences at temperatures between 500 and $600^{\circ} \mathrm{C}$ (Brindley and Brown, 1984).

Thermal metamorphism in the CI chondrites is also apparent in the compositions of secondary phases such as carbonates. Thermally-decomposed $\mathrm{Ca}-\mathrm{Mg}-\mathrm{Fe}-\mathrm{Mn}$ carbonates are abundant in Y-86029 and Y-82162, rather than calcite or dolomite as in other CI chondrites. The thermal decomposition temperatures $\left(\mathrm{T}_{\mathrm{d}}\right)$ for these carbonates have been interpreted as $\sim 470^{\circ} \mathrm{C}$ $\mathrm{T}_{\mathrm{dFeCO} 3}<\mathrm{T}_{\mathrm{dMgCO} 3}<<\mathrm{T}_{\text {dankerite }}<<\mathrm{T}_{\text {ddolomite }}<<\mathrm{T}_{\text {dcalcite }} \sim 900^{\circ} \mathrm{C}$ (Tonui et al., 2014) based on their compositions and previously established thermodynamic models, e.g. Ikornikova and Sheptunov (1973). Ca-Mg-Fe-Mn oxides are also present in these meteorites, most certainly as heating products of Mg-rich carbonate precursors (Ikeda, 1991; Tonui et al., 2014). TEM analysis also shows the presence of submicron grains of pentlandite and pyrrhotite within phyllosilicates and residual olivine in both meteorites. Such grains are absent in unheated CI chondrites suggesting that they result from thermal metamorphism. Tomeoka et al. (1989b) suggested that these sulfides probably form from heating of S-Ni-bearing ferrihydrite but it is important to note that no studies have established the extraterrestrial origin of ferrihydrite (Zolensky et al., 1993). The elongate magnetite crystals with sulfide inclusions observed in Y-86029 and Y-82162 also appear to be heating/oxidation products of sulfides. Other indicators of thermal metamorphism in Y-82162 are also suggested by results of wet chemical analysis, which show distinctly lower $\mathrm{H}_{2} \mathrm{O}$ contents in it than in other CI chondrites (Tomeoka et al., 1989b).

\subsubsection{Regolith Processes Affecting CI Chondrites}

All CI chondrites are breccias, requiring formation by impact processing. Despite their generally hydrous nature, even the CI contain evidence of impact shock. To see this one must look carefully at the regolith breccias, and see past the later aqueous alteration which has generally obscured mineral textures. The Orgueil CI chondrite contains an apparent agglutinate containing melted matrix grading into merely desiccated phyllosilicates (Zolensky et al., 2015). The melt partially devitrified into normally-zoned olivine crystals (Figure 2.5.d) which sit in mesostasis glass. It is interesting that these glasses have partially devitrified to olivine, in contrast to the situation for lunar agglutinates (Lindsay Keller, personal communication, 2014). This could be due to a higher olivine normative composition, and the difficulty of quenching a liquid with an almost pure olivine composition (Gary Lofgren personal communication, 1999).

Zolensky et al. (2015) reported that every $\mathrm{C}$ chondrite examined contains small beads of glass or phyllosilicates which could arguably be microchondrules but when obviously vesicular are more likely a product of impact. Again, unless the origin al glassy state has been preserved, which for such small objects must be a rare occurrence, it is perhaps impossible to ascribe these particular objects to impacts. However, the association with agltuniates supports such an origin for these basea as impact melt spherules. Therefore, we propsoe that these materials will be present in significant quantity on the surfaces of C-class asteroids, where they can be explored by spacecraft such as Hayabusa II and O-Rex.

CI chondrites also display deformation of matrix phyllosilicates which appear similar to that exhibited by clays in permafrost soils (Zolensky, 2005). This texture appears to be evidence of cyclical free-thaw cycles on the parent body. 


\subsubsection{Asteroidal Minerals in CI Chondrites: Non-silicates 2.5.3.4.1 Carbonates in CI Chondrites}

Among the $\mathrm{CI}$ alteration products, the abundant $\mathrm{Fe}-\mathrm{Mg}$-Ca-carbonates are especially important owing to their usefulness for determining aqueous fluid compositions and tempertures, and alteration chronologies (MacDougall et al.,1984; Endress et al.,1996; Lee and Nicholson, 2009). he isotopic work has necessarily focused on dolomite and breunnerite (Endreß and Bischoff, 1996) and the existing Mn-Cr system analyses indicate that the carbonates formed 3$4 \mathrm{Ma}$ after CAIs, with dolomite possibly crystallizing before breunnerite (Hoppe et al., 2007). However, these dates are still preliminary as optimal stardards for these analyses still do not exist (as of this writing several groups are working $\mathrm{n}$ this problem). Ca carbonates (oly recently identified as calcite) are also present, but are relatively scarse (Fredriksson and Kerridge,1988; Richardson, 1978; Johnson and Prinz, 1993; Endreß and Bischoff, 1996, Leee and Nicholson).

Recent work on Orgueil calcites by Lee and Nicholson (2009) suggests that Orgueil (and by implication all other unmetamorphosed CI chondrites) records the following sequence of events: (1) parent body aqueous alteration with at significant depth within the parent body, (2) formation of some calcite (exhibiting twinning and fractures) by impact-related shear stress, (3) corrosion of calcite grains and crystallization of phyllosilicate-magnetite veins accompanying further aqueous alteration, (4) excavation, mixing and reaccretion into a regolith breccia.

Alexander et al. (2015) reported bulk $\mathrm{C}$ abundances, and $\mathrm{C}$ and $\mathrm{O}$ isotopic compositions of carbonates Orgueil and Ivuna. They estimated the $\mathrm{O}$ isotopic composition of the aqueous fluids and the $\mathrm{C}$ isotopic compositions of the $\mathrm{CH}_{4}$ or $\mathrm{CO}$ in these chondrites using clumped isotope carbonate data. The isotopic compositions they estimated for the Orgueil fluid are quite similar to those they suggested for the CMs. They estimated that CI carbonates formed at relatively low temperatures, below $50^{\circ} \mathrm{C}$, which contradicts other estimates for the alteration temperatures for the CIs that range up to $\sim 150^{\circ} \mathrm{C}$ (Zolensky et al. 1989; Clayton and Mayeda 1999). Either the higher alteration temperature estimates are incorrect, or the carbonates formed at lower temperatures, either in the prograde or retrograde regimes, than recorded by the silicate mineralogies and $\mathrm{O}$ isotopes previously used to estimate the alteration temperatures, which assumed chemical equilibrium.

\subsection{Sulfides in CI Chondrites}

As reoorted by all investoigators, pyrrhotite and pentlandite are abundant phases in all CI chondrites (e.g. Zolensky et al., 1993; Tonui et al., 2014).

\subsection{Fe-Oxides in CI Chondrites}

The most important, unambiguously indigenous oxide in CI chondrites is magnetite, which takes the form of framboids, placquettes, and euhedral crystals (Endress M. and Bischoff A., 1996). All unmetamorphosed CI chondrites contain abundant fine grained ferrihydrite within Ferrian saponite, which is typically considered to be a very late stage alteration phase from a oxidixing event on the parent body (Tomeoka and Buseck, 1988). A relative lower abundance of ferrihydrite in Ivuna suggests to some workers that it is the least altered. However, Zolensky et al. (1993) and Zolensky and Gounelle (2001) proposed that all ferrihydrite and the bulk of sulfates in chondrites has a purely terrestrial origin, which would eliminate the need for a late stage highly oxidizing process on the CI parent body. They pointed out that if all the iron in the 
clay-ferrihydrite mixture is converted to ${ }^{2+} \mathrm{Fe}$ the overall composition is a perfect ferrous saponite composition. This and other evidence suggests that the clkay was ioriginally a ferrous saponite, that the ferrous iron was converted to ferric iron after the arrival on earth, resulting in the clay-ferrihydrite mixture. The same process occurs when terrestrial ferrous saponites are moved from an anoxic environment to the surface where they are exposed to the ambient terrestrial atmosphere.

\subsubsection{Asteroidal Minerals in CI Chondrites: Silicates}

\subsection{Phyllosilicates in CI Chondrites}

As mentioned above, in the half that are not thermally metamorphosed CI chondrites consist mainly of an integrowth of serpentine- and saponite-type phyllosilicates with embedded sulfides such as pyrrhotite, pentlandite and cubanite, Fe-Cr oxides such as magnetite and chromite, and iron oxyhydroxides such as ferrihydrite. This material is believed to have formed on or within the parent (Tomeoka et al., 1989b; Ikeda, 1992; Zolensky et al., 1993; Tonui et al., 2014). As also desctibed above, there is evoidence that on the CI parent the saponite contained only ${ }^{2+} \mathrm{Fe}$, whch has since delivery to earth been converted to a mxture of ferroan $\left({ }^{3+} \mathrm{Fe}\right)$ saponite and ferrihydrite (Zolensky et al., 1993). If this proposal is true then a great deal of literature on CI mineralogy and formation processes is erroneous. Unfortunately the transformation of ${ }^{2+} \mathrm{Fe}$ to

${ }^{3+} \mathrm{Fe}$ in saponite is very rapid and so it will probably take a carefully-preserved sample from an asteroid sample return mission to settle the issue.

In the themally metamorphosed CI chondrites phyllosilicates show varying stages of dehydration of phyllosilicates, followed by transformation to fine-grained olivine, as described below. The olivine contains submicron-sized grains of hercynite (Tonui et al. (2014), which appears to be a hallmark of thermal metamorphism. The transformations observed are already described in section 2.5.3.4.2.

\subsubsection{Terrestrial Chemical and Mineralogical Processes Affecting the CI Chondrites}

\subsection{Terrestrial Phases in CI Chondrites}

The CI chondrites are very soft and easily fractured, leading to massive terrstrial contamination. As already described above (section 2.5.3.4.1), CI chondrites contain significant quanties of terrestrial phases, including Ca-sulphates (Zolensky and Gounelle, 2001; Airieau et al., 2005) and ferrihydrite (Zolensky et al., 1993). The sulfate veining in particular served as the textbook example of asteroidal aqueous alteration, until it was shown to be terrestrial in origin. There must also be significant organic contamination in such friable, fractured meteorites. Because of all these problems, any density or porosity measurements of CI chondrites must be viewed with caution (see Corrigan et al., 1997).

\subsubsection{Implications for Exploration of CI Chondrite Parent Bodies}

Selection of sample sites on the B- and C-type target asteroids of the Hayabusa2, OSIRIS-REx, and future sample return missions will need to consider the overall degree of aqueous alteration of the regolith clast population in order to collect samples with the largest proportion of highly altered, phyllosilicate-rich clasts. As with the CM chondrites, CI chondrite aqueous alteration is essentially isochemical - bulk abundances of major and minor elements 
have small variations over small ranges throughout the known range of alteration (Brearley, 2003; King et al., 2015). Consequently, remote analysis of major or minor elements would not be expected to detect compositional variations that indicate or proxy for abundances of volatiles that may influence geotechnical properties. Two of the best correlation relationships between measurable quantities and the overall degree or extent of aqueous alteration are H/Si ratios determined by elemental analysis (Van Schmus and Hayes, 1974) and the modal-abundance ratio of hydrous (phyllosilicate) minerals to anhydrous (primary silicate) minerals determined by quantitative X-ray diffraction (Howard et al., 2011). These ratios will be much higher than for other chondrite types, excepting the metamorphosed CIs (Howard et al., 2009, 2011; Tonui et al., 2014).

A major problem for sample return missions will be preserving the oxidation state of the collected sample, as all previous astromaterials brought to Earth have become xodized such that, for example, the preatmospheric ferrous/ferric ratio has equilibrated wiuth that of terestrial air (Takashi Mikouchi, unpublished data, 2017).

\subsubsection{Physical Properties, Chemical Composition, Mineralogy of CR Chondrites and Processes Affecting these Meteorites}

\subsubsection{Physical Properties of CR chondrites}

As in other carbonaceous chondrite groups, there are only a few measurements of the physical properties of these meteorites, as they generally require destructive analysis. Corrigan et al. (1997) measured the porosity of the matrices (2-8\%) of Al Rais and Renazzo. Macke et al. (2011) measured the physical properties of seven meteorites classified as Renazzo-like (CR) chondrites: Acfer 097, Acfer 270, Al Rais, Dhofar 1432, El Djouf 001, Renazzo, and Tafassasset. They measured the average bulk and grain densities to be $3.11 \pm 0.14 \mathrm{~g} / \mathrm{cm}^{3}$ and $3.42 \pm 0.08 \mathrm{~g} / \mathrm{cm}^{3}$ respectively, the average porosity is $9.5 \% \pm 2.7$ and the average magnetic susceptibility is $\log \chi=5.02 \pm 0.07$. Macke et al. (2011) indicated that the outcomes of terrestrial weathering could not be assessed for CR chondrites, as there are only two observed falls: Renazzo and Al Rais. Because the effect of terrestrial weathering on CR chondrites is more marked than in other groups of carbonaceous chondrites (e.g., Abreu 2016a), the action of this process on the physical properties needs to be investigated. Flynn et al. (2017) noted that weathering of carbonaceous chondrite finds does not produce obvious changes in their physical properties, perhaps because all changes occur very early during the weathering process. One possible reason for this observation is that Macke et al. (2011) focused their studies on CR chondrites that came from hot deserts. Hot desert samples record a different style and a higher intensity of weathering compared with Antarctic finds (e.g., Lee and Bland 2004; Bland et al. 2006). If most changes in physical properties occur after mild terrestrial weathering, as it is the case for ordinary chondrites (e.g., Flynn et al. 2017), patterns are not likely to be observed in extensively weathered CR chondrites from hot deserts. An open question is if and what weathering trends could be observed on the physical properties of cold desert CR chondrites.

Macke et al. (2011) found intra-and inter-sample variability in the physical properties of CR chondrites. They argued that intra-sample heterogeneities might have arisen from brecciation. Since most CR chondrites are breccias, different lithic fragments may have different sets of physical properties. The largest inter-meteorite deviations observed by Macke et al. (2011) may be attributed to the inclusion of anomalous CR2 Al Rais and Tafassasset in the 
averages for the physical properties of this group. Macke et al. (2011) suggested that the large deviations from Al Rais might have resulted from it being a fall. Here, we suggest for an alternative explanation to be explored, namely that the origin of these differences comes from the fact that Al Rais is abnormally depleted in chondrules and enriched in fine-grained materials with respect to other CR chondrites. The chondrule:matrix ratio of Al Rais is 0.46, compared to 1.87 for average CR chondrite (Schrader et al. 2011). Fine-grained chondritic materials are more porous than chondrules. Therefore, it is not surprising that the average porosity of $\mathrm{Al}$ Rais is $25 \%$ \pm 1.6 , which is over twice the average porosity for CRs (Macke et al. 2011). It is unclear which chondrule:matrix ratios are more representative of CR parent bodi(es), if the low ratios from Al Rais or the high ratios from other CR chondrites. Consequently, it may be useful to express physical properties as a function of the chondrule:matrix ratio of the chondrite. On the other hand, we suggest for the physical properties of Tafassasset to be removed from the average CR chondrite values. Gardner-Vandy et al. (2012) presented numerous lines of evidence that Tafassasset is a primitive achondrite, not a CR chondrite. The average bulk and grain densities of Tafassasset are $3.94 \pm 0.05 \mathrm{~g} / \mathrm{cm}^{3}$ and $3.88 \pm 0.02 \mathrm{~g} / \mathrm{cm}^{3}$ respectively, which are about six standard deviations above the CR values (Macke et al. 2011). The average porosity of Tafassasset was calculated to be $-1.5 \pm 1.5$, compared with the average $9.5 \% \pm 2.7$. Such differences are consistent with the fact that Tafassasset is not a CR chondrite. Removing the values for Tafassasset, the average bulk and grain densities for CR chondrites are $2.99 \pm 0.35$ $\mathrm{g} / \mathrm{cm}^{3}$ and $3.36 \pm 0.16 \mathrm{~g} / \mathrm{cm}^{3}$ respectively, the average porosity is $11.23 \% \pm 7.4$. Average CR chondrite densities are slightly higher (as expected from their higher Fe-Ni metal abundances) and porosities are approximately half as for CM chondrites (as expected from the lower chondrule:matrix ratios in $\mathrm{CMs}$ ).

The magnetic susceptibility of CR chondrites is the highest for all the hydrated carbonaceous chondrites and only lower that the values of $\mathrm{EC}$, and the very metal-rich $\mathrm{CH}$ and CB chondrites. Rochette et al. (2008) also analyzed CR chondrites (sixteen in total) including falls, cold and hot desert finds and obtained average magnetization as $\log \chi=5.04 \pm 0.12$ (in $10^{-9} \mathrm{~m}^{3} / \mathrm{kg}$; range 4.51-5.17). These values also included Tafassasset and Kaidun (also reclassified; more details in section below). If the values from these two chondrites are removed from the average, the result is $\log \chi=4.93 \pm 0.23$ (in $10^{-9} \mathrm{~m}^{3} / \mathrm{kg}$; range 4.48-5.17). Removing Tafassasset, the average magnetic susceptibility obtained by Macke et al. (2011) is $\log \chi=4.94$ $\pm 0.18\left(\log \chi *\right.$ in $\left.\log 10^{-9} \mathrm{~m}^{3} / \mathrm{kg}\right)$, which is consistent with the values from (Rochette et al., 2008). These values are comparable/intermediate between CO and CV chondrites (Rochette et al., 2008; Macke et al., 2011; Flynn et al., 2017). Rochette et al., (2008) argued that these magnetic susceptibilities should be interpreted as a low-bound because metallic minerals in CR chondrites have been subjected to significant terrestrial weathering and replacement. Finally, Ibrahim (2012) determined the mean compressional wave velocity for CR chondrites to be 1751 $\pm 611 \mathrm{~m} / \mathrm{s}$ and the average shear wave velocities equal $1751 \pm 611 \mathrm{~m} / \mathrm{s}$. They found the porosity and velocities are anticorrelated.

Aside from the properties listed above, little is known about other physical (e.g., electric, thermal) for either bulk CR chondrites or their components. For example, aside from their diameters, little is known about the physical properties of CR chondrules. There are no estimates of the mass or density of these objects.

\subsubsection{Primary (Nebular) Characteristics of the CR Chondrites}

$\mathrm{CR}$ chondrites are widely considered to be one of the most primitive of the carbonaceous 
chondrite groups (e.g., Kong and Palme 1999; Krot et al. 2005). They contain a record of a wide range of solar nebular processes. For example, CR chondrites have solar abundances for all but the most volatile elements, which are extremely depleted (e.g., Krot et al., 2002). CR chondrites also contain a variety of presolar materials from diverse origins (e.g., Davidson et al. 2014a; Zhao et al. 2013; Floss and Stadermann 2009). Although description of CR chondrite presolar materials is beyond the scope of this chapter, we note that their preservation is consistent with our understanding that this group is indeed very pristine.

CR chondrites contain of abundant chondrules, small and scarce refractory inclusions, and a variety fine-grained materials. Schrader et al. (2011) measured the average modal abundances of the different components for $24 \mathrm{CR}$ chondrites (including two paired samples). They observed that chondrules occupied $\sim 64.6 \%$ of measured areas, fine-grained materials $\sim 35 \%$ (including matrix and dark inclusions), and refractory inclusions were $\sim 0.4 \%$ of the areas measured. Fe-Ni metal grains are abundant and frequently associated with chondrules. Finally, CR chondrites preserve compositionally and isotopically exotic organic matter (e.g., Pizzarello et al. 2012; Glavin et al. this volume and references therein).

The oxygen-isotopic composition of bulk CR chondrites lies on a line below the terrestrial fractionation line with a distinct slope of 0.7 (Weisberg et al., 1995; Clayton and Mayeda, 1999). More recently, Schrader et al. (2011) refined the value of the slope CR line to be $0.70 \pm 0.04$ (2 $\sigma)$ and determined that the $\delta^{17} \mathrm{O}$-intercept is $-2.23 \pm 0.14(2 \sigma)$. These authors noted some spread along the line. This bulk CR chondrite oxygen line is attributed to mixing between ${ }^{16} \mathrm{O}$-poor phyllosilicates and a ${ }^{16} \mathrm{O}$-rich anhydrous silicate component, which are thought to represent at least two distinct reservoirs. Since the CR line represents mixing between a hydrous and an anhydrous component, studies have investigated the relationship between oxygen isotopic composition of CR chondrites and their degree of aqueous alteration. Details of this discussion are presented below.

Alexander et al. (2012) measured H, N, and C bulk and stable isotope composition for 11 unpaired CR chondrites, including the two CR chondrite falls Renazzo and Al Rais and nine Antarctic CRs (EET 92042, EET 96286, GRA 95229, GRO 95577, LAP 02342, LAP 04720, EET 00426, PCA 91082, and QUE 99177). These authors found no differences between falls and finds that suggest that terrestrial weathering influenced the $\mathrm{H}$ content of these meteorites. Bonal et al. (2013) also studied the hydrogen isotopic composition of water in QUE 99177, EET 92042, GRA 95229, Renazzo and Al Rais as well as MET 00426. Finally, Alexander et al. (2013) analyzed an additional three CR chondrites, namely anomalous MIL 090001 and heated GRA 06100 and GRO 03116. Alexander et al. (2012) obtained an average bulk content of $\mathrm{H} \sim 0.55$ wt.\%, ranging from $\mathrm{H} \sim 1.3$ wt.\% in CR1 GRO 95577 and $\mathrm{H} \sim 0.9$ wt.\% in matrix-rich Al Rais, to $\mathrm{H} \sim 0.4$ wt. $\%$ in weakly aqueously altered MET 00426. Because $\mathrm{H}$ can found both in minerals and organic matter, Alexander et al. (2013) calculated the structural "water" (found as structural $\mathrm{OH}$ and $\mathrm{H}$ ) in minerals by subtracting the $\mathrm{H}$ in organics. Structural "water" in CRs range from 2.7-10.9 wt. $\%$ with an average of $\sim 4.2$ wt. $\%$. Hydrogen and the structural "water" contents of bulk CR chondrites are low compared with CM chondrites $\mathrm{H}$ ( 1.2 wt.\%; 0.9-1.5 wt.\% range) and maximum structural "water" (9.4 wt.\% and 7.1-12.2 wt.\% range) calculated by Alexander et al. (2013). Heated CR2s GRA 06100 and GRO 03116 has an $\mathrm{H} \sim 0.1$ wt.\% (Alexander et al. 2013). Values for GRA 06100 are also disproportionally low compared with the average $\mathrm{H}$ content ( 1.0 wt.\%) (Alexander et al. 2012), consequently structural "water" contents are expected to be very low for this meteorite. CR chondrites are $\delta \mathrm{D}$-rich compared to other carbonaceous chondrite groups (e.g., Bonal et al. 2013; Alexander et al. 2012, 2013; Pearson et 
al. 2006). The average $\delta \mathrm{D}$ content for unheated CR chondrites is $639.0 \%$, compared with $\delta \mathrm{D} \sim$ $57.7 \%$ for the CM chondrites (Alexander et al. 2012). Bonal et al. (2013) observed $\delta D$ enrichments as high as $1600 \%$ and high variability in isotopic composition at the micron scale. Heated CR2s also abnormally low $\delta$ D (Alexander et al. 2013). In contrast, Bonal et al. (2013) found no $\delta \mathrm{D}$ trends along the $\mathrm{CR}$ aqueous alteration sequence.

Alexander et al. (2012) measured that the $\mathrm{N}$ and $\mathrm{C}$ content of CR chondrites are low compared to the $\mathrm{CM}$ chondrites. The average $\mathrm{N}$ for the $\mathrm{CR}$ chondrites is $0.08 \mathrm{wt} \%$ versus $\mathrm{N}=$ 0.10 wt.\% in the CMs and the average $\mathrm{C}$ in the CRs is $1.3 \mathrm{wt} . \%$ versus $\mathrm{C}$ in the CMs is 2.0 wt.\%. The average $\delta^{13} \mathrm{C}$ for the CR chondrites is $-4.6 \%$ is comparable with the $\delta^{13} \mathrm{C}$ for the CMs, which is $-2.9 \%$. In contrast, CR chondrites have a high average $\delta^{15} \mathrm{~N}=174.3 \%$, compared with the $\delta^{15} \mathrm{~N}$ of the CM chondrites, which is $56.3 \%$ (Alexander et al. 2012). Heated CR chondrites have low C contents (Alexander et al. 2013).

2.5.4.2.1 Chondrules in CR Chondrites: As in other chondrite groups, CR chondrules are assemblages of ferromagnesian silicate phenocrysts, feldspathic glassy mesostasis, and opaque nodules (Weisberg et al. 1993; Zolensky et al. 1993; Kallemeyn et al. 1994; Kong and Palme 1999; Krot et al. 2002; Schrader et al. 2008, 2011, 2013, 2015, 2018; Rubin 2010; Berlin et al. 2011). Although most CR chondrules are porphyritic olivine (PO) and porphyritic olivine/pyroxene (POP), they present a wide range of mineralogies and textures, including barred olivine (BO), compound $\mathrm{PO} / \mathrm{BO}$, compound $\mathrm{BO} / \mathrm{POP}$, compound porphyritic olivine/radial olivine (PO/RO), and compound porphyritic olivine/granular olivine (PO/GO) (e.g., Weisberg et al. 1993; Zolensky et al. 1993; Kallemeyn et al. 1994; Kong and Palme 1999; Krot et al. 2002; Schrader et al. 2008, 2011, 2015). Based on previous studies (e.g., McSween, 1985; Hewins et al., 2005), Schrader et al. (2015) argued that the preponderance of PO and POP chondrules indicates that few of chondrule precursors were fully melted or that the CR region of chondrule formation was possibly dusty.

Texturally, CR chondrules range from well-rounded, extensively melted chondrules with large silicate phenocrysts and opaque grains to irregular, slightly melted, fine-grained objects. Some CR chondrules have multiple concentric layers containing micro-porphyritic and granular silicates, as well as Fe-Ni metal grains (e.g., Krot et al. 2002; Ebel et al., 2008; Rubin 2010). These outer layers are termed igneous rims. Rubin (2010) measured the average thickness of igneous rims around $\mathrm{CR}$ chondrules to be 270 microns, which is think compared with rims in CK chondrules (190 microns), ordinary chondrules (160 microns), and CM, CO, EH and EL chondrules (30-60 microns). The compositional differences in Fe-Ni metal and their implications for chondrule formation are discussed below. Ebel et al. (2008) used 3D tomographic images to image the olivine-pyroxene layering sequence in layered chondrules. They argued that because olivine condenses at higher temperatures than pyroxene, the olivine-pyroxene layering sequence is consistent with high-temperature equilibration with a solar gas. In addition to chondrules with igneous rims, some CR chondrules are compound objects consisting of two chondrules either in contact or nested within each other (e.g., Rubin 2010). Finally, some CR chondrules have agglomeratic, or partially melted grains ranging in sizes from 1-80 microns (e.g., Schrader et al. 2018).

Multiple studies have reported that CR chondrules are large compared with chondrules in other chondritic groups, in particular CM and CO chondrites (e.g., Bischoff et al. 1993; Kallemeyn et al. 1994; Zanda et al. 2005; Rubin 2010; Friedrich et al. 2015). Kallemeyn et al. (1994) reported the average radius of CR chondrules to be $\sim 700 \mu \mathrm{m}(\mathrm{n}=146$ across $4 \mathrm{CR}$ 
chondrites). However, Friedrich et al. (2015) cautioned that given the textural complexity of CR chondrules, it is not clear that all authors use the same delineation for the chondrule edge. Zanda et al. (2005) argued that the grain size of silicate phenocrysts increases with the degree to which chondrule precursors melted in the nebula. Weisberg et al. (1993) suggested that both the chondrule cores and igneous rims originated from the same reservoirs. Rubin (2010) explained the large chondrule diameters of CR chondrules (as well as chondrules in CV and CK chondrites) as the product of re-melting in slow-cooling, dust-rich environments, in which chondrules accumulated dust layers on their surfaces.

In addition to mineralogical and textural classifications, $C R$ chondrules can be subdivided into three types based on their chemical compositions, which are thought to have been established under different nebular conditions. The $\mathrm{Fe} /(\mathrm{Fe}+\mathrm{Mg})$ ratio of chondrule ferromagnesian silicates has been used to subdivide them into type I and type II chondrules. Type I chondrules are reduced, $\mathrm{MgO}$-rich, $\mathrm{FeO}$-poor $(\mathrm{Fe} /(\mathrm{Fe}+\mathrm{Mg})<10 \%)$. Type II chondrules are $\mathrm{FeO}$-rich $(\mathrm{Fe} /(\mathrm{Fe}+\mathrm{Mg})>10 \%)$. In the $\mathrm{CR}$ chondrites, there is a sharp gap in compositions between these two types that is not as prominent as in other carbonaceous chondrite groups. Schrader et al. (2015) interpreted this paucity in composition as evidence that chondrules in CR chondrites formed under distinct conditions and that no chondrules from other groups are present in these meteorites. Type I chondrules are generally the most abundant component of CR chondrites, accounting for $\sim 63.1 \%$ and ranging from $30-74 \%$ of the total meteorites' areas (Schrader et al. 2011). Type II chondrules represent approximately $2.3 \%$ of CR chondrite areas and range widely from 0.1 to $5.6 \%$ (Schrader et al. 2011). A third group of CR chondrules contains high-Ca, low-Ca pyroxenes, and $\mathrm{Mg}$-bearing anorthitic plagioclase and are termed Anorthite-Rich Chondrules or ARCs (e.g., Krot et al. 2002). ARCs are the least common of the three groups, accounting for $\sim 0.5 \%$ and ranging from $0-1.6 \%$ of the total CR areas (Schrader et al. 2011).

Type I Chondrules: Type I chondrules are Fe-Ni metal-rich, vary in size, generally ranging from $400-1000 \mu \mathrm{m}$, but can reach up to $3 \mathrm{~mm}$ in apparent diameter. Type I chondrules are commonly multilayered; with cores dominated by forsteritic olivine and in some cases low-Ca pyroxene phenocrysts (e.g., Weisberg et al. 1993; Kallemeyn et al. 1994; Krot et al. 2002; Schrader et al. 2008, 2013, 2015; Tenner et al., 2013, 2015). Both cores and layers main contain silicates, Fe-Ni metal, and feldspathic glass mesostasis, which may be partially to completely replaced by phyllosilicates, most commonly serpentine and chlorite. Igneous rims often have silica-rich outer layers. Furthermore, pyroxenes have higher contents of Fs, $\mathrm{MnO}$, and $\mathrm{Cr}_{2} \mathrm{O}_{3}$ and mesostasis have higher albite and orthoclase contents (e.g., Krot et al. 2002). Unlike type I chondrules in CM, CO, OC, R, EH, and EL chondrites, sulfides are scarce in type I CR chondrules (e.g. Rubin 2010).

The low abundance of sulfur in type I chondrules has been explained by multiple heating events (e.g., Schrader et al. 2008; Rubin 2010; Jacquet et al. 2013). However, there is active discussion as to the mechanism and conditions under which $\mathrm{S}$ losses occurred. Schrader et al. (2008) suggested that low these $\mathrm{FeO}$ chondrules formed by heating gas and dust in a reducing and non-sulfidizing (low $f \mathrm{O}_{2}$ and $f \mathrm{~S}_{2}$ ) region. In contrast, Rubin (2010) argued that the low $\mathrm{S}$ contents of type I CR chondrules resulted from slow cooling during re-melting, which allowed migration of S chondrules' surfaces and eventual evaporative losses. Jacquet et al. (2013) also suggested that type I CR chondrules underwent at least two nebular heating events. The first event was argued to have lasted $>1$ day and resulted both in equilibration between chondrules 
and $\mathrm{Fe}-\mathrm{Ni}$ metal and in evaporative loss of $\mathrm{Fe}$ from the chondrule. The first heating event would be followed by accretion of a fine-grained mantle on the chondrule surface. Like Rubin (2010), Jacquet et al. (2013) suggested that a second heating event would explain the formation of igneous rims. However, unlike Rubin (2010) who suggested for the second heating event to be slow, Jacquet et al. (2013) argued that the second heating event was rapid, at least compared to elemental diffusion and limited to the outside of the chondrule.

These characteristics of Fe-metal in chondrules are discussed below.

Fe-Ni Metal in Type I CR Chondrules: Fe-Ni metal in CR chondrites has been studied in detail. These phases are the reservoir of approximately a third of the iron in CRs (Campbell et al. 2005). Fe-Ni metal is found in the interiors of chondrule phenocrysts and mesostasis ( 45-50\%), chondrule margins ( 15\%), and embedded in matrix (e.g., Lee et al. 1992; Connolly et al. 2001; Ebel et al. 2008). Based on X-ray microtomography, Fe-Ni represents up to $37 \%$ of the volume of type I chondrules, though there is significant variability (Ebel et al. 2008). For example, nonporphyritic chondrules do not generally contain metal. Based on minor element compositions, Zanda et al. (1994) showed that CR Fe-Ni metal in all petrologic settings had a common origin. Kong et al. (1999) argued that Fe-Ni metal grains outside chondrules probably were also derived from chondrules. Later LA-ICP-MS trace element studies have supported this hypothesis. Jacquet et al. (2013) studied nine different CRs and obtained largely unfractionated refractory siderophile elements and depleted volatile $\mathrm{Au}, \mathrm{Cu}, \mathrm{Ag}$, and $\mathrm{S}$. Despite their common origin, the characteristics of Fe-Ni metal in CRs appear to vary with petrologic setting and degree of chondrule melting.

Compositional and textural CR Fe-Ni metal characteristics vary with petrographic setting (e.g., Lee et al. 1991; Weisberg et al. 1993; Connolly et al. 2001; Humayun et al. 2002;

Campbell et al. 2005). For example, Fe-Ni metal in chondrule interior metal generally has higher refractory siderophile element contents (e.g., Co, Ni, Re, Os, W, Ir, Ru, Mo, Pt) than metal on margins (e.g., Jacquet et al. 2013). Conversely, Fe-Ni metal located on chondrule margins has relatively higher abundances of volatile elements (e.g., Fe, $\mathrm{Cu}, \mathrm{Au}$ ). Campbell et al. (2005) noted that small Fe-Ni metal grains that are poikilitically enclosed in $\mathrm{MgO}$-rich silicates have high, correlated concentrations of $\mathrm{Ni}$ and P and low Fe and Cr. Wasson and Rubin (2010) observed that Fe-Ni metal composition is uniform within individual concentric shells in chondrules. Metal grain sizes also tend to be smaller in igneous rims around chondrules compared to chondrule interiors and margins. However, it is important to note that Ebel et al. (2008) determined that the two-dimensional arrangement of Fe-Ni metal grains - on which these correlations rely - may not be representative of the three dimensional distribution of metal.

The texture of CR Fe-Ni metal grains also varies with the degree to which their chondrule hosts underwent melting as measured by the shape and silicate phenocryst sizes (e.g., Zanda et al. 2002). Round chondrule outlines and large silicate phenocrysts are argued to result from more extensive melting than chondrules with finer-phenocrysts. Zanda et al. (2002) reported that Fe-Ni metal is stochastically distributed throughout type I chondrules with small silicate phenocryst size. In contrast, type I chondrules with large phenocrysts and a round outlines have welldeveloped shells made out of coarse-grained Fe-Ni metal. In addition, in extensively melted chondrules, olivines have higher forsterite contents and Fe-Ni metal grains have higher Ni and P.

There are several hypotheses for the origin of Fe-Ni metal: (1) reduction during thermal metamorphism in the CR parent body(s); (2) direct condensation from a nebular gas; (3) evaporation and recondensation during chondrule formation; and (4) segregation of immiscible 
metallic Fe melts from chondrule melts in the nebula. To test the validity of Fe-Ni metal formational hypotheses, their petrologic textures, settings, minor and trace elements have been studied.

While current discussion about the origin of Fe-Ni metal center around a nebular setting, Lee et al. (1992) proposed that Fe-Ni metal formed by reduction of $\mathrm{Fe}^{2+}$ during thermal metamorphism. The parent-body reduction hypothesis contradicts more recent evidence that thermal metamorphism played only a minimal role in the history of CR chondrites (e.g., Wasson and Rubin 2010; Schrader et al. 2015; Abreu 2016). In addition, Wasson and Rubin (2010) noted that the negative correlation the observed between the $\mathrm{Ni}$ in the metallic shell and olivine Fa contents in the host silicates argue against the idea of thermally-driven in-situ reduction.

Based on the abundances of $\mathrm{Ni}, \mathrm{Cr}$ and $\mathrm{P}$ Fe-Ni metal, early studies suggested that this metal condensed directly from the nebula, prior to silicate formation (Wood 1967; Grossman and Olsen 1974). Later studies showed that some CRs show a positive, linear correlation between Co and $\mathrm{Ni}$ and approximately solar ratio of $\sim 0.046$ (Lee et al. 1992; Weisberg et al. 1993; Connolly et al. 2001; Jacquet et al. 2013). Weisberg et al. (1993) interpreted this trend as a result from thermodynamically-controlled condensation nebular conditions. It is noteworthy that several studies show that the $\mathrm{Co} / \mathrm{Ni}$ ratios are not constant within individual or across different CRs (e.g., Zanda et al., 1994, 2002; Wasson and Rubin 2010; Abreu 2016). Multiple lines of evidence have been used to rule out the direct nebular condensation hypothesis has been ruled out, including thermodynamic equilibrium of metallic Fe melts under nebular conditions, the presence of a graphite layer on Fe-Ni metal surfaces, and trace siderophile element abundances (e.g., Kong et al. 1999; Connolly et al. 2001; Humayun et al. 2002; Jacquet et al. 2013). Kong et al. (1999) observed that Fe-Ni metal had low-Ni contents and was embedded in graphite layer. They argued that scenarios for nebular condensation could either reproduce the low-Ni composition of Fe-Ni metal or the presence of the graphite layer, but not both observations. The Ni contents would require isolation of metal from the nebular gas at high temperature. In contrast, graphite could only be stable at temperatures $600 \mathrm{~K}$ (at $10^{-5}$ bar) in the nebula, requiring long exposure of $\mathrm{Fe}-\mathrm{Ni}$ metal to the nebular gas at low temperatures. Finally, Humayun et al. (2002) found a linear correlation between $\mathrm{Ni} / \mathrm{Fe}$ and $\mathrm{Pd} / \mathrm{Fe}$ in $\mathrm{CR} 2 \mathrm{Renazzo}$, which cannot be explained by the following order of condensation from a gas of solar composition: Re, Os, Ir, Ru, Mo, $\mathrm{Pt}>\mathrm{Ni}>\mathrm{Co}>\mathrm{Pd} \approx \mathrm{Fe}>\mathrm{Au}>\mathrm{Cu}>\mathrm{Ga}>\mathrm{Ge}$.

The last two hypotheses propose that Fe-Ni metal formation occurred as a consequence or as a part of chondrule-forming events. These two hypotheses are not mutually exclusive. In fact, Connolly et al. (2001) suggested that they were both at play, depending on the petrologic setting of the Fe-Ni metal, rim "metal" forming by the evaporation/recondensation and chondrule metal forming by separation of immiscible metal-silicate chondrule melts. It has been suggested that at least of the CR Fe-Ni metal formed by devolatilization concurrently with the formation of immiscible metal-silicate chondrule metals and subsequent recondensation from the resulting vapor, which would be depleted in refractory siderophiles (Kong et al. 1999; Kong and Palme 1999; Connolly et al. 2001). This formational scenario can explain that rim Fe-Ni "metal" is depleted in refractory siderophiles and complementarity between the $\mathrm{Au}, \mathrm{As}, \mathrm{Sb}$, and $\mathrm{Ga}$ contents of coarse Fe-Ni metal and non-magnetic Fe-Ni matrix phases. The carrier of this nonmagnetic Fe-Ni signature is likely to be the Fe-Ni sulfides that were later identified to be one of the dominant components of CR matrix (Abreu and Brearley 2010; Abreu 2016). However, Campbell et al. (2005) argued that such scenario is unlikely because elements such as $\mathrm{Au}$ and $\mathrm{Cu}$ 
may not be easily mobilized from the melt under the kind of reducing conditions needed to form Fe-Ni metal. Jacquet et al. (2013) also argued against recondensation of metal vapor.

Finally, based on depletion of volatile siderophile elements, authors argued that large $\mathrm{Fe}-\mathrm{Ni}$ metal formed by reduction of $\mathrm{FeO}$ and separation from the silicate fraction of chondrule melts (Connolly et al., 1994; Zanda et al., 1994; Connolly 2001; Campbell et al. 2002, 2005). Additional constraints have been placed on the melt origin hypothesis. Connolly et al. (1994) suggested that reduction was assisted by the presence of carbon. Graphite-assisted reduction is supported by the fact that graphite is spatially associated with Fe-Ni metal (Kong et al. 1999). Some studies explained the Co-Ni trend observed in some CR metal in terms of oxidation/reduction reactions based on the observation that Fe-Ni metal and silicates appear to be redox equilibrium with each other within individual chondrules (Lee et al. 1992; Zanda et al., 1994). Because $\mathrm{Ni}$ and Co are slightly more refractory than Fe, the initial metal composition would have been in enriched in $\mathrm{Co}$ and $\mathrm{Ni}$ with respect to the last condensates. Finally, Wasson and Rubin (2010) suggested that separation of Ni and Co from Fe-metal occurred during volatilization associated with chondrule formation at low ambient nebular temperatures.

The texture and composition of $\mathrm{Fe}-\mathrm{Ni}$ have been used to shed light on the nature of the $\mathrm{Fe}-\mathrm{Ni}$ metal precursors. Some authors suggested that Fe-Ni metal formed by decomposition of a precursor Fe-Ni sulfide (e.g., Zanda et al. 1997, 2002; Hewins et al. 1997). Zanda et al. (2002) argued that the correlation between degree of melting and the composition of silicates and Fe-Ni metal support this hypothesis. In contrast, Jacquet et al. (2013) suggested that Fe-Ni metal precursors resemble the least-melted chondrules.

Although possible that the presence of discontinuous metal shells is an artifact of 2D petrographic observations, the formation of continuous Fe-Ni metal shell is unlikely to be an artifact and merits an explanation. Multiple hypotheses have been proposed to explain the arrangement of Fe-Ni metal within chondrules. According to Wood and McSween (1977), the shell-like distribution of Fe-Ni metal within chondrules resulted from addition and/or condensation of successive layers in the nebula. Alternatively, it has been suggested that metallic Fe was mobilized to the periphery of chondrules via centrifugal force differential, acting on immiscible fluids of different density (i.e., metallic Fe versus forsteritic melts) (e.g. Grossman and Wasson 1985; Tsuchiyama et al. 2000; Kong et al. 1999; Kong and Palme 1999; Humayun et al. 2002). Wasson and Rubin (2010) also argued that centrifugal forces could not reproduce the shell-like, concentric structures that Fe-Ni globules form within many chondrules. Instead, according to Wasson and Rubin (2010), surface tension resulted in the mobilization of the metallic Fe melt across channels. The melt then redistributed itself on the surface of the silicate melt via interfacial tension. Presumably, the rate of cooling of the mobilized metallic Fe melt was slow compared to Ni diffusion, which would result in the uniform composition of individual concentric shells. However, model proposed by Wasson and Rubin (2010) is not consistent with the trace element data obtained by Connolly et al. (2001). Wasson and Rubin (2010) argued that the variations in PGE contents within shell observed by Connolly et al. (2001) were artifacts of ion microprobe analysis. However, it is noteworthy that similar PGE patterns were independently reproduced using ICP-MS (Humayun et al. 2002; Campbell et al. (2002).

Finally, with the exception of Renazzo and Al Rais, all CR chondrites are finds and consequently were affected by terrestrial weathering. Fe-Ni metal grains are some of the materials most prominently affected by Antarctic weathering. A discussion of the effect of weathering on metal grains is presented in the terrestrial non-silicates section below. 


\section{Mesostasis in Type I CR Chondrules:}

Harju et al. (2014) examined the mesostasis in 16 finds classified as CR chondrites, including samples from hot and cold deserts. Of the 16 samples, three Saharan CR chondrites (Acfer 394, Acfer 395, and Acfer 399) and two Antarctic samples (EET 87847 and EET 92062) are paired with each other. Since that study, one of the meteorites analyzed, MIL 07513 has been reclassified, reducing the sample suite to 12 unique CR chondrites. Of those 12 unique samples, $6 \mathrm{CR}$ chondrites contain at least one chondrule that has preserved clear, unaltered mesostasis. The samples containing the highest numbers of chondrules with clear mesostasis are Gao-Guenie b, LAP 02342, and EET 92062 (Harju et al. 2014). Isotropic glass was only identified in pristine CR QUE 99177 (Harju et al. 2014). Not all members of each of pairing groups contain clear mesostasis, revealing heterogeneities in their asteroidal and/or terrestrial histories. Harju et al. (2014) measured the composition the mesostasis of two chondrules, one POP chondrule in each LAP 02342, and EET 92062. They found that mesostasis in the LAP 02342 chondrule was enriched in $\mathrm{Na}$ and depleted in $\mathrm{Ca}, \mathrm{Ti}$, and $\mathrm{Cr}$ near the boundary between mesostasis and finegrained matrix. These trends were attributed to parent body aqueous alteration.

In pristine CR chondrites MET 00426 and QUE 99177, Tenner et al. (2015) described the mineralogy of mesostasis in 48 type I chondrules as generally heterogeneous in texture, typically consisting of fine-to-coarse grained pyroxene and plagioclase. Some occurrences reported were glassy. Tenner et al. (2015) used EDS to measure the composition of the mesostasis in one region of recrystallized mesostasis $\left(\mathrm{SiO}_{2}, \mathrm{Al}_{2} \mathrm{O}_{3}, \mathrm{FeO}, \mathrm{MgO}, \mathrm{CaO}\right.$, and $\mathrm{Na}_{2} \mathrm{O}$ contents of 56, 24, $1,3,12$, and 2.5 wt.\%, respectively) and one region of glassy mesostasis $\left(\mathrm{SiO}_{2}, \mathrm{Al}_{2} \mathrm{O}_{3}, \mathrm{FeO}\right.$, $\mathrm{MgO}, \mathrm{CaO}$, and $\mathrm{Na}_{2} \mathrm{O}$ contents $68,15,4,1.5,6.5$, and 3.5 wt. $\%$, respectively).

Abreu (2016b) collected 320 electron microprobe point analyses from 5-8 type I and II chondrules in each of nine, unpaired Antarctic CR chondrites spanning the extent of aqueous alteration recorded by the CR parent body (EET 92062, LAP 02342, GRA 95229, MIL 07525, EET 96259, anomalous MIL 090001, LAP 04516, LAP 04720, and GRO 95577). No compositional trends have emerged from mesostasis in either chondrule type. She reported that $\mathrm{Ca}, \mathrm{Na}, \mathrm{K}, \mathrm{Cr}$, and $\mathrm{S}$ were not related to the amounts of secondary minerals, to water content measured by (Abreu 2016a), to their total oxide contents, or to their Antarctic weathering category.

\section{Type I Chondrule Isotopic Studies:}

Several studies have focused on the oxygen isotopic composition of type I CR chondrules (e.g., Krot et al. 2006b; Schrader et al. 2013, 2014; Tenner et al. 2015). Authors have argued that aqueous alteration of the meteorites studied has not modified their oxygen isotopes of their chondrules (e.g., Schrader et al. 2013). Krot et al. (2006b) measured the O-isotopic composition of all major phases in nine chondrules from three Antarctic CR chondrites, EET 92042, GRA 95229, and MAC 87320. These chondrules plotted near the carbonaceous chondrite anhydrous mineral (CCAM) line (Krot et al. 2006b). Krot et al. (2006b) found that with the exception of relict grains, chondrules are isotopically homogeneous with all minerals and mesostasis from each chondrule plotting in small clusters at $\Delta^{17} \mathrm{O} \sim \pm 2 \%$. Interchondrule variations range from $\Delta{ }^{17} \mathrm{O}=0$ to $-5 \%$. Krot et al. (2006b) suggested that the oxygen isotopic composition of CR chondrules was established during melting and isotopic homogenization of precursor material with a ${ }^{16} \mathrm{O}$-poor gas.

Results from more recent oxygen isotopic studies are generally consistent with work by Krot et al. (2006). Using secondary ion mass spectrometry (SIMS), Schrader et al. (2013) studied 
olivine grains (21 analyses) in eight type I CR chondrules from GRA 95229, GRA 06100, and QUE 99177 - of which the bulk oxygen isotopic composition of GRA 06100 is ${ }^{16} \mathrm{O}$-poor and QUE 99177 is ${ }^{16}$ O-rich. They argued that each chondrule acted as an individual igneous system with its own isotopic composition.

On a follow-up study, Schrader et al. (2014) analyzed a total of ten type I chondrules from Gao-Guenie (b), GRA 95229, PCA 91082, and Shişr 033 - seven barred olivine (BO) chondrules and three silica-bearing porphyritic chondrules. Schrader et al. (2014) found that olivine grains oxygen-isotope compositions plotted along a slope-1 line. BO chondrule olivines oxygen isotopic variations are small (i.e., $\Delta^{17} \mathrm{O} \sim 2.5 \%$ - Schrader et al. 2014). These observations indicate that olivine grains in type I BO chondrules have similar major and minor element and Oisotope compositions to those of olivine in porphyritic chondrules. They argued against the chondrule formation model in which porphyritic chondrules formed in transient planetesimals based on these similarities in isotopic compositions. In addition, they suggested that even BO chondrules, which were at one point completely melted, only record partial equilibration of nebular gas.

Tenner et al. (2015) used SIMS to measure the O-isotopic composition of olivine, pyroxene, and plagioclase in 45 type I chondrules $(\mathrm{Mg \#}=94.2-99.2)$ from pristine QUE 99177 and MET 00426. They found an inverse correlation between $\Delta^{17} \mathrm{O}$ and $\mathrm{Mg}$-numbers, which they suggested had implications for the processes forming type II chondrules. Furthermore, Tenner et al. (2015) used the trend established between $\Delta^{17} \mathrm{O}$ and $\mathrm{Mg}$-numbers to constrain the amount of dust and/or $\mathrm{H}_{2} \mathrm{O}$ enhancements needed for type I CR chondrules to form. They calculated 100-200x dust enrichments and that these dust precursors had 0-0.8 x CI chondrite water atomic abundance. However, it is important to note that a more recent study of seven other CR chondrules did not reproduce the correlation between $\Delta^{17} \mathrm{O}$ and $\mathrm{Mg}$-numbers (Schrader et al. 2017). Consistent with prior studies, Tenner et al. (2015) observed that $\Delta^{17} \mathrm{O}$ were nearly homogeneous for all minerals analyzed. However, unlike Schrader et al. (2014), Tenner et al. (2015) argued that the chondrule melts exchanged oxygen isotopes efficiently with the surrounding nebular gas.

Studies of the Al-Mg systematics of CR chondrules show few ${ }^{26} \mathrm{Mg}$ excess (e.g., Krot and Keil, 2002; Hutcheon et al., 2009; Nagashima et al., 2014; Olsen et al., 2016; Schrader et al., 2017). Some authors have interpreted the low number of chondrules with ${ }^{26} \mathrm{Mg}$ excess as evidence that $\mathrm{CR}$ chondrules are younger than their counterparts in other chondritic groups, while others have attributed this observation to spatial variations of the ${ }^{26} \mathrm{Al} /{ }^{27} \mathrm{Al}$ ratio within the solar protoplanetary disk. For example, a recent study by Schrader et al. (2017), which simultaneously measured the oxygen and the Al-Mg systematics of seven type I porphyritic CR chondrules from GRA 95229, GRO 03116, PCA 91082, and QUE 99177, found no ${ }^{26} \mathrm{Mg}$ excesses in these chondrules. Chondrules in this study were selected because their host shows minimal signs that thermal metamorphism, definitely insufficient to disrupt the systematics. Schrader et al. (2017) estimated that only approximately $32 \%$ of chondrules in CR chondrites show resolvable excesses of ${ }^{26} \mathrm{Mg}$. Using other chronometers, they suggested that $\mathrm{CR}$ chondrites contain three different generations of chondrules. Budde et al. (2018) have recently applied the short-lived ${ }^{182} \mathrm{Hf}-{ }^{182} \mathrm{~W}$ chronometer to metal obtaining chondrule ages that $3.6 \pm 0.6$ million years (Ma) after the formation, which also support the hypothesis of late CR chondrule formation. On the other hand, Olsen et al. (2016) interpreted the variability in ${ }^{26} \mathrm{Mg}$ as the result of $\mathrm{Mg}$-isotope heterogeneity of the chondrule precursors. Along with the low variability in ${ }^{54} \mathrm{Cr}$ values observed in CR chondrules, Olsen et al. (2016) argued that these chondrules must have formed in a spatially constrained region possibly located in the outer solar system. 
Type II Chondrules: Oxidized, FeO-rich chondrules are less abundant than type I chondrules. They often occur as fragments (41\% of type II chondrules - Schrader et al. 2015). Schrader et al. (2008) suggested that preferential breakdown of type II chondrules occurred because either because type-II chondrules originated in a more dynamically active or denser nebular region or because these chondrules were more brittle and/or porous than type-Is.

The Fe-Mg-Mn systematics of silicates in CR type II chondrules has been studied in detail (Berlin et al. 2011; Schrader et al. 2015). Berlin et al. (2011) observed that chondrules from different chondritic groups could be differentiated based on the trends defined by their Fe/Mn versus Fe/Mg ratios. However, Schrader et al. (2015) collected a much larger dataset that showed significant overlap among groups. According to Schrader et al. (2015), the Fe-Mg-Mn systematics of these silicates may be attributed to a variety of factors, including (1) individual type II chondrules behaving as individual igneous systems formed under distinct oxygen fugacities (Schrader et al. 2013); (2) formation from variable precursors (e.g., Berlin et al. 2011); (3) formation at different cooling rates (e.g., Jones and Lofgreen 1993; Berlin et al. 2011); or (4) experiencing complete/incomplete condensation.

According to Connolly et al. (2008), type II chondrules formed flash-heating of both dust and pre-existing type I chondrules under higher than canonical $f \mathrm{O}_{2}$ and $f \mathrm{~S}_{2}$. They tested this hypothesis by measuring the oxygen isotopic composition of type II CR chondrules (see below in discussion of isotopes - Connolly and Huss 2010). Schrader et al. (2015) observed compositional overlaps between $\mathrm{FeO}$ and minor elements (e.g., $\mathrm{MnO}, \mathrm{Cr}_{2} \mathrm{O}_{3}$, and $\mathrm{CaO}$ ) in type I silicate phenocrysts compared with $\mathrm{FeO}$-poor silicate relicts located in type II chondrules. These overlaps suggest that while origin of type II chondrules from type I chondrule materials is possible, it is also possible that both types originated from similar precursors. In either case, additional precursors are necessary to account for compositional differences between these two types of chondrules.

Type II Chondrule Opaque Assemblages:

Type II chondrules have a distinct opaque population (Schrader et al. 2008, 2015). In most cases (with the exception of GRA 06100 - Abreu and Bullock 2013), opaques in type II chondrules are more complex than in type I chondrules, containing $\mathrm{Fe}-\mathrm{Ni}$ metal, troilite, pentlandite, magnetite, and phosphates (e.g., Schrader et al. 2008, 2015). Although Schrader et al. (2008) indirectly identified tochilinite as part of these assemblages; a later study determined that these occurrences were instead sub-micron mixtures of magnetite, pyrrhotite, and pentlandite (Schrader et al. 2015). Schrader et al. (2008) initially argued that the opaque assemblages in type II chondrules formed by a two-stage process, starting in the nebula and continuing in the CR parent body. First, Fe-Ni metal and troilite would form by flashheating/cooling condensation and corrosion reactions during chondrule formation. They envisioned that this first stage was dynamically violent, explaining the fragmentation of chondrules described above (Schrader et al. 2008). Formation of secondary phases (i.e., magnetite and phosphates) would occur by replacement of Fe-Ni metal in the CR parent body at temperatures at or around 300C. Results from experimental studies support this hypothesis (Schrader and Lauretta 2010). Schrader and Lauretta (2010) reproduced the mineralogy of the type II chondrule opaque assemblages by reacting $\mathrm{Co}$, Cr, P-bearing, Fe-Ni alloys with $\mathrm{H}_{2}-\mathrm{H}_{2} \mathrm{O}-$ $\mathrm{CO}-\mathrm{CO}_{2}-\mathrm{H}_{2} \mathrm{~S}$ gas mixtures at $1000 \mathrm{C}$ and cooling them at $3000 \mathrm{C} /$ hour rate. This experimental study and later TEM observations showed that pentlandite in type II chondrules may be nebular (Schrader and Lauretta 2010; Schrader et al. 2015). 
Type II Chondrule Mesostasis:

Burger and Brearley $(2004,2005)$ studied partially altered mesostasis of two type II CR chondrules in paired EET 87770 and EET 92105. The observed that while unaltered mesostasis regions were albite normative; $\mathrm{Na}$ and $\mathrm{K}$ had been leached from altered mesostasis. Altered mesostasis and adjacent matrix, however, was enriched in Ca. Burger and Brearley (2005) added that $\mathrm{Ca}$ and $\mathrm{P}$ mobilization occurred early in the alteration process. This observation was confirmed by later FIB/TEM observations. Brearley and Burger (2009) analyzed the transition between glassy and altered mesostasis EET92105. They observed that even in the glassy mesostasis alteration proceeded by the removal of $\mathrm{Ca}$ and $\mathrm{P}$ through the formation of $\mathrm{Ca}$ phosphate veins. In a more altered mesostasis region, phyllosilicates had replaced the glass.

Little data for the bulk chemical composition of type II CR chondrules is available. Tenner et al. (2015) described the mesostasis in 3 type II chondrules in MET 00426 and QUE 99177 as glassy. They used EDS to measure the composition of the mesostasis in one region of glassy mesostasis $\left(\mathrm{SiO}_{2}, \mathrm{Al}_{2} \mathrm{O}_{3}, \mathrm{FeO}, \mathrm{MgO}, \mathrm{CaO}\right.$, and $\mathrm{Na}_{2} \mathrm{O}$ contents 60,8 to 14, 13, 2 to 5, 0.5, and 8 to $12 \mathrm{wt} . \%$, respectively).

\section{Type II Chondrules Isotopes:}

In general, the oxygen isotopic composition of individual type II CR chondrules falls within the same trend as the type I chondrules, although they show larger $\Delta^{17} \mathrm{O}$ values. Authors have argued that aqueous alteration of the meteorites studied has not modified their oxygen isotopes of their chondrules (e.g., Schrader et al. 2013). Krot et al. (2006b) measured the O-isotopic composition of mesostasis and olivine in one type II chondrule from MAC 87320, which plotted either near the CCAM or near the terrestrial fractionation line. Connolly and Huss (2010) analyzed the oxygen isotopic composition of 11 type II, $\left(\mathrm{Fa}_{16-53}\right)$ porphyritic chondrules from Renazzo, EET 92011, and MAC 87320. Similar to type I CR chondrules, minerals within individual chondrules were nearly isotopically homogenous, but distinct from one another. Connolly and Huss (2010) found that type II CR chondrules tend to be more $\delta^{17} \mathrm{O}$ - and $\delta^{18} \mathrm{O}$ rich than type-I chondrules and have $\Delta^{17} \mathrm{O}$ ranging from $-2.0 \pm 0.7$ to $0.5 \pm 0.7$. They also suggested that relict grains in type II chondrules, which had a lighter oxygen isotopic composition than their hosts, originate from type-I CR chondrules. Connolly and Huss (2010) hypothesized that type II chondrule formation was aided by the combination of dust enhancements and the addition of ${ }^{16} \mathrm{O}$-poor $\left({ }^{17} \mathrm{O}\right.$ and ${ }^{18} \mathrm{O}$-rich) ice to precursors.

Schrader et al. (2013) measured the isotopic composition of olivines (43 analyses) in 12 type II (Fa $\mathrm{Fa}_{12.2-53.8)}$ CR chondrules located in GRA 95229, GRA 06100, and QUE 99177, of which 8 chondrules were relict-free. Texturally, analyses included POP and compound BO/POP and radial olivine/POP chondrules. Olivines in relict-grain-free chondrules had O-isotope compositions range in $\delta^{17} \mathrm{O}$ and $\delta^{18} \mathrm{O}$ by approximately $2 \%$, and in $\Delta^{17} \mathrm{O} \sim 3 \%$. In contrast, Schrader et al. (2013) found that relict-grain-bearing type II chondrules had much larger ranges in $\delta^{17} \mathrm{O}$ and $\delta^{18} \mathrm{O}(\sim 14 \%)$ and in $\Delta^{17} \mathrm{O}(\sim 7 \%)$. Schrader et al. (2013) calculated that relictgrain-free type-II chondrules formed under $\mathrm{H}_{2} \mathrm{O} / \mathrm{H}_{2}$ ratios between 230 and 740 times solar; whereas, relict grain-bearing type-II chondrules formed under $\mathrm{H}_{2} \mathrm{O} / \mathrm{H}_{2}$ ratios between 350 and 510 times solar. In general, these type II chondrules were more ${ }^{16} \mathrm{O}$-rich than reported in previous studies. Schrader et al. (2013) also suggested that the type II CR chondrule dust precursor had a higher S content than the type I chondrule precursor. 
Schrader et al. (2013) also studied the relationship between the FeO content of these olivines and their minor elements $\left(\mathrm{MnO}, \mathrm{Cr}_{2} \mathrm{O} 3, \mathrm{Na}_{2} \mathrm{O}\right)$ to ascertain whether type I chondrules are the precursor materials for type II chondrules. They concluded that the relationship between these elements indicate that these relationship is unlikely. This is in contrast with a more recent study by Tenner et al. (2015). Tenner et al. (2015) studied the O-isotopic composition of 3 type II chondrules (Mg\# = 53-63) from pristine QUE 99177 and MET 00426. Simultaneously, they used type I chondrule trends between $\Delta^{17} \mathrm{O}$ and $\mathrm{Mg}$-numbers to support the hypothesis presented by Connolly and Huss (2010), in which type I chondrules are the precursors for type II chondrules. They also hinted that the oxygen isotopic trends suggest that type II chondrules might have formed in a different environment and by a different process compared with type I chondrules. It is noteworthy that although Schrader et al. (2013) reported a relationship between the oxidation state of olivine and its O-isotope composition, Schrader et al. (2017) did not confirm the relationship between $\Delta^{17} \mathrm{O}$ and $\mathrm{Mg}$-numbers. Schrader et al. (2014) studied four barred olivine (BO) and one porphyritic olivine pyroxene (POP) chondrule (olivine) chondrules from GaoGuenie (b), GRA 95229, PCA 91082, and Shişr 033. They found that the oxygen-isotope compositions of olivines in type II BO chondrules span a slightly larger range in $\Delta^{17} \mathrm{O} \sim 2.1 \%$ o compared with olivines in BO type I chondrules (Schrader et al. 2014). Based on their observations, Schrader et al. $(2013,2014)$ argued that there was exchange, but no homogenization between chondrules and the surrounding nebular gas. Finally, Schrader et al. (2017) observed a ${ }^{26} \mathrm{Mg}$ excess in plagioclase while simultaneously measuring the oxygen and the Al-Mg systematics of one type II porphyritic CR chondrule from GRO 03116, suggesting that at least some type II CR chondrules might have formed earlier than some type I CR chondrules.

Anorthite-Rich Chondrules (ARCs): ARCs are relatively scarce chondrules ( $<1$ vol.\%). These chondrules are mineralogically distinct from type I and type II chondrules, although some ARCs have regions that are similar to type I chondrules (e.g., Krot et al. 2002). ARCs are dominated by pyroxene and olivine phenocrysts, which generally have high Mg-numbers and also contain $\mathrm{Fe}-$ $\mathrm{Ni}$ metal nodules and crystalline mesostasis composed of silica, anorthite and high-Ca pyroxene (Krot and Keil 2002). However, unlike type I chondrules, ARCs have interstitial anorthite and Al-Ti-Cr-rich low-Ca and high-Ca pyroxenes, and in some cases contain refractory inclusions (Krot and Keil 2002). This association is the basis of the argument that ARCs formed by melting of the spinel-anorthite-pyroxene CAIs mixed with ferromagnesian precursors compositionally similar to Type I chondrules (Krot and Keil, 2002; Krot et al. 2006b). Based on their high abundances of moderately-volatile elements such as Cr, Mn and Si, Krot and Keil (2002) argued that ARCs could not have been formed by volatilization of the type I chondrule precursors or by melting of the refractory materials only.

\section{Anorthite-Rich Chondrule Isotopes:}

Krot et al. (2006) measured the isotopic composition of different minerals (olivine, orthorpyroxene, clinopyroxene, anorthite, and spinel) in five ARC. Unlike type I and type II chondrules, which they found to be essentially isotopically homogeneous, Krot et al. (2006b) reported that two out of five ARCs are isotopically heterogeneous, with large variations in $\Delta^{17} \mathrm{O}$ ( -6 to $-15 \%$ and -2 to $-11 \%$ ). They attributed these variations to incomplete melting of precursors, which they identified as CAIs. 
Nagashima et al. (2014) measured the Al-Mg isotope systematics in fourteen CR chondrules from Acfer 311, EET 92042, EET 92174, El Djouf 001, GRA 95229, and NWA 721 using SIMS. Most of these chondrules were Al-rich, but five type two type II chondrules and I were also included in this study. Of these chondrules, six show evidence of in-situ decay of ${ }^{26} \mathrm{Al}$. However, Nagashima et al. (2014) noted that ${ }^{26} \mathrm{Mg}$ excesses in CR chondrules are small compared with excesses in other pristine chondrites, such as ordinary chondrites LL3.0, CO3.0 chondrites, and unique carbonaceous chondrite Acfer 094. Nagashima et al. (2014) attributed the small ${ }^{26} \mathrm{Mg}$ excesses to $\mathrm{CR}$ chondrule formation occurring over a million years after chondrules in the other pristine chondrites.

2.5.4.2.2. Fine-Grained Materials in CR Chondrites: By volume, the second most abundant component of CR chondrites is matrix. CR matrix is complex, presenting a variety of different occurrences including interchondrule matrix, dark inclusions (DI), "feathered" matrix, and smooth rims (e.g., Abreu and Brearley 2010; Harju et al. 2014; Wasson and Rubin 2014; Abreu 2016a). The least altered CR matrices are dominated by amorphous Fe-Mg silicates, Fenanophase sulfides, and minor olivine and pyroxene (Abreu and Brearley 2010; Le Guillou and Brearley 2014; Le Guillou et al. 2015; Abreu 2016a). Even in the least altered CRs, matrices have been extensively hydrated (Alexander et al. 2013; Bonal et al. 2013; Beck et al. 2014; Garenne et al. 2014; Le Guillou et al. 2015). Hydration has only resulted in extensive recrystallization in the most altered CRs, in which nebular phases have been replaced to varying degrees by micron to sub-micron Fe-Mg phyllosilicates, magnetite, partly oxidized Fe-sulfides, and tochilinite (Abreu 2007; Abreu and Brearley 2010; Harju et al. 2014; Le Guillou et al. 2015; Abreu 2016a). No systematic textural changes are observed in weakly to moderately altered CR matrices (Abreu 2007; Abreu and Brearley 2010; Harju et al. 2014; Le Guillou et al. 2015; Abreu 2016a).

\section{Interchondrule Matrix:}

Abreu (2007, 2016a) observed that the majority of fine-grained materials in CR chondrites showed no direct textural association with chondrules, but simply occupied the space between them. These fine-grained materials were term the interchondrule matrix, which is variable in texture and composition as seen in Table 2.XX. The size of interchondrule matrix regions is very variable in CR chondrites; some areas are occupied by relatively large (500-800 $\mu \mathrm{m})$ units of interchondrule matrix while in other regions chondrules are barely separated from each other.

Abreu (2007) reported that there are texturally different types of interchondrule matrix can be distinguished in pristine and weakly altered CR chondrites: (1) exclusively fine-grained (crystals $<5 \mu \mathrm{m}$ - Fig. 2.XX), which may contain mineral fragments, (2) matrix clasts, and (3) distinct Ferich regions (Fig. 2.XX). The interchondrule matrix contains a mixture of sub-micron materials (e.g., amorphous Fe-Mg silicates, $\mathrm{Fe}(\mathrm{Ni})$ sulfides, Fe-oxides and in some cases calcite), mineral fragments (generally forsteritic olivine), chondrule fragments, metal, and coarser prismatic to rounded Fe(Ni)-sulfides (e.g., Zolensky et al. 1993; Abreu and Brearley 2010; Harju et al. 2014; Wasson and Rubin 2014; Abreu 2016a). Clasts of fine-grained matrix material that appear texturally and mineralogically distinct from the adjacent matrix have also been identified in CR chondrites, being particularly abundant in GRA 95229. These clasts show clear boundaries from the adjacent interchondrule matrix and are diverse in terms of size, shape, and texture. Some areas of matrix appear very bright in BSE images in comparison to other fine-grained regions. These regions are irregular and particularly Fe-rich and extend for several hundreds of microns. 
Fe-rich regions contain Fe-Ni metal nodules, chondrule fragments, abundant Fe-sulfides of various shapes, and calcite. Iron and S maps X-ray maps show that Fe-enrichment is partly caused by the presence of a particularly high abundance of rounded sulfides ranging from submicron to tens of microns, which are disseminated throughout the matrix (Abreu 2007). In addition to the high abundance of sulfides in these regions, the fine-grained silicate-rich component of the clast also contains elevated Fe contents. Abreu (2016a) studied one such area located in MIL 07525 using TEM. The feathery texture resulted from the presence of interpenetrating units dominated by the Fe-phyllosilicate hisingerite, which was attributed to Antarctic leaching of octahedral Mg ions from asteroidal Fe-rich saponite. Magnetite is rare in interchondrule matrices of pristine and weakly altered CR chondrites. Calcite is rare in all regions in most CR matrices, with the exception of CR1 GRO 95577 (e.g., Morlok and Libourel, 2013).

\section{Fine-Grained Rims in CR Chondrites:}

The presence of fine-grained rims has been reported in some CRs chondrites (e.g., Abreu 2007; Abreu and Brearley 2010; Tyra 2013). However, fine-grained rims are significantly less abundant than in CM chondrites. The paucity of fine-grained rims around most CR chondrules has been confirmed in more recent studies (e.g., Wasson and Rubin 2014; Abreu 2016a). In pristine and weakly altered CR chondrites, many continuous, well-defined rims are up to $200 \mu \mathrm{m}$ in thickness and consist of homogeneous, fine-grained materials decorated with magnetite and sulfide crystals $(<20 \mu \mathrm{m})$ on their peripheries (Abreu 2007; Abreu and Brearley 2010). The finegrained materials contain sub-micron silicates and sulfides that generally cannot be resolved by SEM. Sulfides (troilite with minor amounts of Ni) and magnetites on the periphery of the finegrained materials are generally $<20 \mu \mathrm{m}$ in diameter. Wasson and Rubin (2014) argued that since some of the least altered carbonaceous chondrites in our collections have chondrules that are generally fine-grained rim-free, that fine-grained rim might not have formed in the solar nebula, but instead might be the product of asteroidal aqueous alteration.

In addition to fine-grained rims, Harju et al. (2014) identified fine-grained occurrences around chondrules in pristine CR LAP 02342. They termed these materials smooth-rims based on their featureless texture in back-scattered electron images and argued they were dominated by phyllosilicates, which formed as a consequence of aqueous alteration. However, Abreu (2016a) performed direct mineralogical characterization of one of these rims using TEM and found that amorphous materials dominated it, suggesting that they may have a more similar origin as the rest of CR matrix.

\section{Carbonaceous Materials in CR Chondrite Matrices:}

Isotopic studies have shown that organic materials in CR chondrites are indeed very primitive and their organics consist of a highly heterogeneous mixture of materials rich in heavy isotopes (interstellar) and materials that do not show these enrichments (e.g., Busemann et al. 2006; Glavin et al., this volume). Organic matter from MET 00426, QUE 99177, EET 92042, and Renazzo has been studied in situ using a combination of microbeam techniques (e.g., Abreu, 2007; Floss et al. 2014; Le Guillou and Brearley, 2014 Le Guillou et al. 2014). Abreu (2007) characterized organics in MET 00426, QUE 99177, and EET 92042. Le Guillou and Brearley (2014) also examined the characteristics of organics in MET 00426. Le Guillou et al. (2014) analyzed the characteristics of carbonaceous matter in Renazzo using NanoSIMS, synchrotronbased scanning transmission X-ray microscopy (STXM), and TEM. 
Abreu (2007) observed that carbonaceous materials in MET 00426, QUE 99177, and EET 92042 were distributed at the nanoscale in a variety of different textural occurrences. Le Guillou and Brearley (2014) documented additional textural occurrences, namely the presence of nanoglobule-like features, elongated veins, and networks of sub-rounded grains connected by cracks. They attributed these textural differences to differences in the degree of aqueous alteration of organics, which has originally been accreted into the CR parent body as ice-organics particles. Organic materials show a very low degree of graphitization (e.g., Abreu 2007; Le Guillou and Brearley 2014). Abreu (2007) reported mineralogical associations between organics and nano-sulfides, carbides, magnetites, and phyllosilicates, as well as the presence of isolated C-rich regions. Le Guillou and Brearley (2014) confirmed these mineralogical associations and in addition identified associations with tochilinite. Organic matter in Renazzo was located in cracks between amorphous silicate regions and/or associated with phyllosilicates (Guillou et al. 2014). They observed that Renazzo contains individual grains of molecularly homogeneous organic materials, but in some cases had heterogeneous morphologies and D/H ratio. They suggested that these grains probably contained insoluble organic compounds. Based on comparative studies with CI (Orgueil) and CM (Murchison) chondrites, Le Guillou et al. (2014) suggested that organic materials were altered during aqueous alteration.

Abreu (2007) suggested that some of the materials in CR chondrites must have formed or been reprocessed after interstellar synthesis, favoring FTT synthesis as a mechanism playing an important role in the synthesis of organics in the solar nebula based on the mineral-organic spatial relationships observed in MET 00426, QUE 99177, and EET 92042. Abreu (2007) reported that nano-phases (i.e. sulfides and carbides) are invariably associated with carbonaceous materials, a texture that must have resulted from FTT reactions in the solar nebula. FischerTropsch type synthesis was probably enhanced by the large surface-to-volume ratio of nano phases observed in MET 00426, QUE 99177, and EET 92042. Catalysis must have occurred below $\sim 300^{\circ} \mathrm{C}$ probably during nebular cooling after formation of sulfides and amorphous silicates by high temperature evaporation and recondensation events. This solar carbonaceous material later mixed with an interstellar component, giving rise to the exotic isotopic signatures characteristic of CR chondrites.

Floss et al. (2014) performed coordinated NanoSIMS, Auger, and FIB-TEM analyzes of organic matter in QUE 99177 and MET 00426. Although Floss and Stadermann (2009) had previously reported the presence of both ${ }^{13} \mathrm{C}$-enrich and ${ }^{13} \mathrm{C}$-depleted organic regions in these meteorites, Floss et al. (2014) found that organic matter had solar carbon isotopic compositions but large enrichments in ${ }^{15} \mathrm{~N}$ compared solar (average 195\%o) - in agreement with IOM values obtained by Alexander et al. (2007). Larger ${ }^{15} \mathrm{~N}$ enrichments were observed in QUE 99177. Although no all organic-rich regions present isotopic anomalies, ${ }^{15} \mathrm{~N}$ enrichments are highest in concentrated organics hotspots and lower in more diffuse regions of organics (Floss et al. 2014). Auger microscopy revealed that organic grains had both nanoglobule and irregular morphologies and TEM analysis showed a high degree of crystallographic disorder (Floss et al. 2014). Floss et al. (2014) found no correlations between isotopic composition and morphology, petrographic association, or elemental composition. They suggested that the diffuse regions formed by redistribution of organics during aqueous alteration.

\subsection{Refractory Inclusions in CR Chondrites:}

Because refractory inclusions are not very common in CRs (CAIs $\sim 0.4-<1$ vol.\% - Aléon et al. 2002; Schrader et al. 2011), they have not received as much attention as their counterparts in 
$\mathrm{CV}$ chondrites. Refractory inclusion is an umbrella term for both calcium-aluminum-rich refractory inclusions (CAIs) and amoeboid olivine aggregates (AOAs).

CR CAIs are fairly small ( $<500 \mathrm{um})$ and irregularly shaped, and most of them are fragmented (Aléon et al. 2002; Krot et al. 2017). Based on the mineralogy and petrography, Aléon et al. (2002) divided the CAIs in CR chondrites into grossite +/- hibonite-rich, melilite-rich, pyroxeneanorthite-rich, and spinel-pyroxene-melilite aggregates. Further, they distinguished two textural varieties of the pyroxene anorthite-rich CAls, most CAIs were irregularly shaped, while some had igneous textures. Aléon et al. (2002) measured the oxygen isotopic composition of minerals in 27 CAIs located in seven CR chondrites: El Djouf 001, EET 87747, EET 92041, GRA 95229, MET 00426, PCA 91082, and Renazzo. They determined that CR CAIs are ${ }^{16} \mathrm{O}$ rich. The $\Delta^{17} \mathrm{O}$ for hibonite, melilite, spinel, pyroxene, and anorthite was $<-22 \%$. Unlike CAIs in other groups of carbonaceous chondrites, CR CAIs appear to have escaped both aqueous alteration and thermal metamorphism (Aléon et al. 2002).

CR AOAs are aggregates of anhedral forsterite grains and refractory inclusions composed of spinel, pyroxene and anorthite, and in some Fe-Ni metal nodules (Aléon et al. 2002; Weisberg et al. 2004). Fe-Ni metal in CR AOAs has similar composition as metal in type I chondrules (Weisberg et al. 2004). Texturally, CR AOAs have irregular shapes, can be fluffy, and lack the glassy mesostasis characteristic of chondrules (Weisberg et al. 2004). Aléon et al. (2002) measured the $24 \%_{0}<\Delta^{17} \mathrm{O}<-20 \%$ in forsterite, spinel, anorthite, and pyroxene in CR AOAs. According to Weisberg et al. (2004), CR AOAs record a complex nebular history, beginning with gas-solid condensation, reactions of minerals with the nebular gas, small degrees of melting, and sintering of the assemblage. Weisberg et al. (2004) also described a textural association between an AOA and a type A CAI suggestive of heating during the same nebular event.

\subsection{Asteroidal Minerals: Non-silicates}

\subsection{Carbonates in CR Chondrites}

Carbonates are only abundant in the most altered CR chondrite. Carbonates in weakly to moderately altered CR chondrites are predominantly Ca-rich. Although a magnesium siderite has been identified in CR2 Acfer 059/El Djouf 001 and in primitive CR MET 00426 (Endress et al., 1994; Brunner and Brearley, 2011). Micron and sub-micron occurrences of Ca-carbonates have been reported in these meteorites (Abreu and Brearley 2010; Abreu 2016a).

Carbonates are most abundant in the most altered CR1 chondrite, GRO 95577. Carbonates distributed either throughout the matrix or along the periphery of chondrule pseudomorphs (Tyra, 2013). Tyra (2013) reported the presence of calcite (2.9 vol.\%; 10-30 $\mu \mathrm{m}$; anhedral), "siderite" (Mg-, Mn-bearing; 1.0 vol.\%; 1-10 $\mu \mathrm{m}$; anhedral), and minor dolomite $(<2 \mu \mathrm{m}$; blocky) in this meteorite. These carbonates are texturally associated with one another, with opaques (Fe-oxides and Fe-sulfides), or with phyllosilicates.

Using SIMS, Jilly-Rehak et al. (2018) measured the oxygen isotopic composition of calcite and dolomite in the matrices of Al Rais, EET 92159, GRO 95777, Renazzo, QUE 99177, and MIL 090292 (it is possible that this last meteorite may not be a CR chondrite). They found that the $\delta^{18} \mathrm{O}$ of calcite ranged from 9 to $35 \%$ and plotted on a line with slope $\sim 0.64$ (Jilly-Rehak et al. 2018). Jilly-Rehak et al. (2018) found no clear relationship between degree of aqueous alteration recorded by each meteorite. The CR1 GRO 95577 experienced little to no fluid evolution during carbonate precipitation, which was attributed to high water-to-rock ratio of the sample (Jilly-Rehak et al. 2018). In addition, Jilly-Rehak et al. (2018) identified dolomite in dark 
inclusions found in EET 92159 and Renazzo. The $\delta^{18} \mathrm{O}$ of dolomites ranged from approximately 23 to $27 \%$.

Jilly-Rehak et al. (2017) measured the ${ }^{53} \mathrm{Mn}-{ }^{53} \mathrm{Cr}$ systematics of carbonates from three different CR-chondrite lithologies from Renazzo and extensively altered GRO 95577. JillyRehak et al. (2017) found that Renazzo carbonates formed approximately 4.3-5.3 Myr after the formation of CAIs in CV chondrites, which overlaps with the time for aqueous alteration of Tagish Lake, CI, and CM chondrites. However, calcite grains in GRO 95577 is even younger, having formed approximately 12.6 Myr (Jilly-Rehak et al. 2017) and 24 Myr (Tyra, 2013) after CAI formation.

\subsection{Sulfides and Tochilinite in CR Chondrites}

Although some authors have argued for asteroidal origin, CR Fe(Ni) sulfides are generally thought to have formed in solar nebula (e.g., Schrader et al. 2008). Coarse-grained Fe-sulfides are generally located in the interior and surface of chondrules (Wasson 1996; Schrader et al. 2008). Nanophase pyrrhotite $(<10 \mathrm{~nm}-100 \mu \mathrm{m})$ and pentlandite $(<10 \mathrm{~nm}-10 \mu \mathrm{m})$ are common accessory phases in CR matrices (Lee et al. 1992; Abreu and Brearley 2010; Abreu 2016a). In CR2 chondrites, sub-micron and nanophase sulfides have been partially oxidized (Abreu 2016a). In fact, recent reports have identified nanophase tochilinite in some of the most pristine CR chondrite matrices (e.g., Le Guillou and Brearley 2014; Abreu 2016a). Le Guillou and Brearley (2014) reported spatial relationships among pentlandite, tochilinite, and serpentine, including layers of tochilinite on sulfide surfaces. Based on their occurrence as overgrowth of magnetite and association with siderite grains, Tyra (2013) argued that at least some of the coarse-grained Fe-sulfides in GRO 95577 matrix are asteroidal.

\subsection{Fe-Oxides in CR Chondrites}

Fe-oxides have been identified in all CR chondrites. In weakly to moderately altered CR chondrites, Fe-oxides are fine-grained $(<50 \mu \mathrm{m})$ and scarce. In some cases they are found as independent rounded or platelet grains disseminated throughout the matrix, while in others they form framboids (e.g., Kallemeyn et al. 1994; Abreu and Brearley 2010; Tyra 2013; Abreu 2016). In some of the least altered CR chondrites, rounded Fe-oxides are found decorating the periphery of fine-grained rims around chondrules (Abreu and Brearley 2010). The abundance of Fe-oxides increases drastically with aqueous alteration as surmised by their abundance in CR1 GRO 95577. Fe-oxides are comparatively large $(100 \mu \mathrm{m}-1 \mathrm{~mm})$, texturally and mineralogically complex (Weisberg and Huber, 2007; Morlok and Libourel, 2013; Tyra, 2013). Morlok and Libourel (2013) argued that these grains formed by alteration of Fe-Ni metal nodules. Finally, Jilly-Rehak et al. (2018) measured the $\delta^{18} \mathrm{O}$ to range between -18 and 5\% in Al Rais, EET 92159, GRO 95777, Renazzo, QUE 99177, and MIL 090292 (this last meteorite may not be a CR chondrite). Magnetite formation has long been recognized as an indicator of aqueous alteration in chondritic meteorites (e.g. CR - Kallemeyn et al. 1994 and CI - Kerridge et al. 1979). Kallemeyn et al. (1994) argued that magnetite formed during hydrothermal alteration at temperatures between 350-540 K. As precursor phases were replaced during aqueous alteration, Fe was released and entered magnetite and phyllosilicates. Preferential formation of either phyllosilicates or magnetite probably depends on the relative thermodynamic stabilities of these phases. 


\subsection{Asteroidal Minerals in CR Chondrites: Silicates}

\subsection{Phyllosilicates in CR Chondrite Matrices}

Although CR chondrites share many similarities with CI and CM chondrites, the matrices of these meteorites contain variable amounts of amorphous Fe-Mg silicates (e.g. Abreu and Brearley 2010, Le Guillou et al. 2015; Abreu 2016a). Transmission electron microscopy studies have shown that aqueous alteration of CR matrices occurs by progressive, yet incomplete replacement of amorphous Fe- $\mathrm{Mg}$ silicates by phyllosilicates (Abreu and Brearley 2010, Le Guillou et al. 2015a; Abreu 2016a). Abreu and Brearley (2010) characterized the silicates in the matrices of some of the most pristine CR matrices in QUE 99177 and MET 00426. Le Guillou et al. (2015a) analyzed sub-micron silicates in eight CR chondrites (QUE 99177, EET 87770, EET 92042, LAP 02342, GRA 95229, Renazzo, Al Rais, and GRO 95577). Abreu (2016a) reported the compositions and occurrences of Fe-Mg silicates in nine Antarctic CR chondrites (EET 96259, GRA 95229, GRO 95577, GRO 03116, LAP 02342, LAP 04516, LAP 04720, MIL 07525, and anomalous MIL 090001). Even the least aqueously altered CR chondrites; QUE 99177, MET 00426, and LAP 02342 show some replacement of amorphous Fe-Mg silicates by Fe-Mg phyllosilicates (Abreu 2007; Abreu and Brearley 2010; Le Guillou and Brearley 2014; Floss et al. 2014; Le Guillou et al. 2015a; Abreu 2016a). Le Guillou et al. (2014) observed both fine-grained Fe-Mg phyllosilicates (composition between serpentine and saponite) and coarser grained $(\sim 100 \mathrm{~nm})$ Fe-rich cronstedtite in the matrix of Renazzo.

In CR matrices there is a textural and compositional (oxides and water contents) continuum between amorphous Fe-Mg silicates and Fe-Mg phyllosilicates that suggests that phyllosilicates formed by replacement of amorphous silicates (e.g., Abreu, 2007, Abreu and Brearley, 2010; Le Guillou et al. 2015a; Abreu, 2016a). The compositions of these materials overlap each other, as does their water contents (e.g., Abreu, 2007, Abreu and Brearley, 2010; Le Guillou and Brearley 2014; Le Guillou et al. 2015a; Abreu, 2016a). Le Guillou et al. (2015a) used a combination of STXM and TEM data to determine that amorphous Fe-Mg silicates are hydrated and have high $\mathrm{Fe}^{3+} / \sum \mathrm{Fe}$ ratios $(68-78 \%)$ in weakly to moderately altered CRs. They reported that the composition was intermediate between serpentine and saponite in weakly to moderately altered CRs (QUE 99177, EET 87770, EET 92042, LAP 02342, GRA 95229, Renazzo), whereas altered Al Rais and GRO 95577 were richer in Mg and Si. In contrast with other reports of LAP 02342 (i.e., Wasson and Rubin 2009; Harju et al. 2014; Abreu 2016a), Le Guillou et al. (2015a) observed that LAP 02342 shows signs of aqueous alteration and extensive replacement of amorphous Fe-Mg silicates. Le Guillou et al. (2015a) reported that phyllosilicates in LAP 02342 have higher Mg\# and are coarser grained than in other CR2 chondrites. However, no images are available to allow comparisons with other reports (i.e., Abreu 2016a).

Intriguingly, amorphous $\mathrm{Fe}-\mathrm{Mg}$ silicates, which appear to be the most reactive phases in these matrices are also one of the last ones to be fully replaced. The matrix of the most altered

CR, GRO 95577, still contains some amorphous Fe-Mg silicates along with a few relict metal, olivine, and pyroxene phenocrysts (Abreu 2016a; Howard et al. 2015). The mineralogical assemblage of matrices becomes more variable and heterogeneous as new and coarser phyllosilicates are formed (Abreu 2016a).

\subsection{Phyllosilicates Replacing CR Chondrite Mesostasis}


While there are numerous reports that CR chondrule mesostasis has been partially to completely replaced by phyllosilicates (e.g., Zolensky et al. 1993; Harju et al. 2014), there are few detailed studies of the characteristics of mesostasis phyllosilicates or the mechanism of replacement. Keller (2011) reported mesostasis is replaced by serpentine and chlorite in anomalous CR MIL 090001. Brearley and Burger (2009) observed Fe-rich aluminous serpentine replacing chondrule mesostasis in EET 92105.

\subsection{Phyllosilicates Replacing CR Chondrite Chondrule}

Phenocrysts

Replacement of silicate phenocrysts has only been observed in the most altered CR chondrite, GRO 95577. In this meteorite, most chondrules have been replaced by phyllosilicate pseudomorphs, ranging in sizes from less than 0.05-2 mm (e.g., Weisberg and Huber 2007; Tyra 2013; Harju et al. 2014; Abreu 2016). However, there are no studies detailed studied describing the mineralogical characteristics of these phyllosilicates.

\subsection{Asteroidal Chemical and Mineralogical Processes Affecting the CR Chondrites}

\subsection{Models of Aqueous Alteration Processes Affecting the CR Chondrites}

The CR group contains some of the least and the most aqueously altered carbonaceous chondrites in our collections. However, a large gap in samples exists between CR chondrites recording intermediate and very extensive degrees of aqueous alteration (e.g., Alexander et al. 2013; Harju et al. 2014; Howard et al. 2015; Abreu 2016a). Most members of the group are classified as petrologic type 2; although there is a single example of petrologic type 1 (e.g., Weisberg and Prinz 2001; Perronnet and Zolensky 2006) and type 3 (e.g., Abreu and Brearley 2010; Wasson and Rubin 2014; Le Guillou and Brearley 2014; Le Guillou et al. 2015a,b; Abreu 2016a) have been identified. The degree of aqueous alteration of CR chondrules is highly variable. Chondrule phenocrysts and Fe-Ni metal nodules in type $3 \mathrm{CR}$ chondrites have largely escaped aqueous alteration. Chondrules in CR chondrites range from anhydrous to containing hydrous mesostasis, to being completely replaced by serpentine, saponite and chlorite (Ichikawa and Ikeda 1995; Weisberg et al. 1995). Nebular minerals in CR2 matrices have been replaced to different extents by phyllosilicates (dominantly serpentine), framboidal magnetite, and minor calcite (Weisberg et al. 1993; Abreu 2016a). In heavily altered CR chondrites, such as Al Rais and GRO 95577, secondary alteration has caused extensive replacement of chondrule mesostases and matrix by phyllosilicates (Weisberg et al. 1993). Chondrules in CR chondrites show heavy isotopic enrichments in the $\mathrm{O}$ consistent with aqueous alteration (Krot et al. 2002). However, unaltered Fe-Ni metal, occurring as individual crystals and associated with chondrules, has been preserved (Krot et al. 2002).

Aqueous alteration of CR chondrites appears to have taken place at low temperatures and in multiple stages (e.g., Le Guillou et al. 2015a; Jilly-Rehak et al. 2018). In the earliest stages of aqueous alteration, Fe-Mg phyllosilicates replaced nebular amorphous Fe-Mg silicates. Le Guillou et al. (2015a) suggested that aqueous alteration of CR matrices is a two-step process that begins with hydration and oxidation of nebular amorphous $\mathrm{Fe}^{2+}-\mathrm{Mg}$ silicates to produce metastable, predominantly $\mathrm{Fe}^{3+}$ amorphous $\mathrm{Fe}-\mathrm{Mg}$ silicates, followed by formation of $\mathrm{Fe}-\mathrm{Mg}$ phyllosilicates with lower $\mathrm{Fe}^{3+} / \sum \mathrm{Fe}$ ratios (e.g., 55\% in extensively altered Al Rais and GRO 
95577). According to Le Guillou et al. (2015a), both of these processes occurred in an asteroidal environment. Le Guillou et al. (2015b) carried out experimental studies that suggest that these early replacement reactions took place at approximately $90^{\circ} \mathrm{C}$. Jilly-Rehak et al. (2018) used the oxygen isotopic fractionation that they observed between carbonates and magnetites to calculate the precipitation temperatures of these phases to be $\sim 60^{\circ} \mathrm{C}$. For closed-system alteration of the CR parent body, Jilly-Rehak et al. (2018) estimated that its global temperature during alteration was $\sim 55-88^{\circ} \mathrm{C}$, which is generally consistent with the temperatures for phyllosilicate formation. Jilly-Rehak et al. (2018) argued that the variations in oxygen isotopic compositions of matrix minerals in CR chondrite resulted from aqueous alteration processes affecting the CR parent body in a very heterogeneous manner. Jilly-Rehak et al. (2018) suggested that their observations are inconsistent with carbonates in CR chondrites forming from a single, uniform fluid. However, they did argue that $\mathrm{O}$-isotopic composition of the altering fluids evolved from approximately the Al Rais water composition of $\Delta^{17} \mathrm{O} \sim 1 \%$ and $\delta^{18} \mathrm{O} \sim 10 \%$, and became increasingly ${ }^{16} \mathrm{O}$-enriched toward a final fluid composition of $\Delta^{17} \mathrm{O} \sim-1.2 \%$ and $\delta^{18} \mathrm{O} \sim-15 \%$.

The ${ }^{53} \mathrm{Mn}-{ }^{53} \mathrm{Cr}$ systematics of carbonates has been used to constrain the duration of aqueous alteration in the CR parent body (Tyra, 2013; Jilly-Rehak et al., 2017). While JillyRehak et al. (2017) placed aqueous alteration of the CR parent body in the same window as other hydrated chondrites, GRO 95577 preserved very young carbonates (Tyra, 2013; Jilly-Rehak et al. 2017). Jilly-Rehak et al. (2017) proposed two possible scenarios, (a) a large enough (30-50 $\mathrm{km}$ radius) $\mathrm{CR}$ parent body in which heat from radiogenic decay was retained for approximately 8 Myr after accretion or (b) episodic formation of secondary phases as a consequence of impactdriven metamorphism.

Determination of the degree to which aqueous alteration have affected CR components is an area of active research. Three scales have been devised to quantify the degree of aqueous alteration of CRs, with a particular emphasis on assigning sub-types to the CR2 chondrites. Alexander et al. (2013) observed that there was a linear relationship between bulk H, C, and N elemental and isotopic composition of CMs and CRs and used this correlation to assign petrologic subgroups. Harju et al. (2014) postulated petrologic criteria to classify the CRs: (1) presence/abundance of chondrules containing altered mesostasis, (2) presence/abundance of magnetite, (3) replacement of primary silicate phenocrysts by phyllosilicates, (4) replacement of Fe-Ni metal with oxides, (5) abundance of phyllosilicates in matrix and chondrule rims, and (6) S-content of the matrix. Finally, Howard et al. (2015a) used the ratio of phyllosilicates to anhydrous silicates (i.e., olivine and pyroxene) to assign petrologic sub-types. Although the classification schemes proposed by Alexander et al. (2013) and Howard et al. (2015a,b) are generally consistent with each other, these schemes often disagree with petrologic classifications (Harju et al., 2014) and sub-micron observations (e.g., Abreu 2016a).

Abreu (2016a) argued that of the differences in classification assignments may be explained by the distinguishing features of CRs, which have very fine-grained matrices dominated by Fe$\mathrm{Mg}$ amorphous silicates, by their abundant Fe-Ni metal, and extensive brecciation. Mineralogybased classification, such us the scheme proposed by Harju et al. (2014), has proved particularly challenging for weakly to moderately altered CRs because incipient mineral replacement and elemental mobilization arising from aqueous alteration only affected the most susceptible primary phases, which are generally located in the matrix (Abreu 2016a). Secondary matrix phases are extremely fine-grained (generally sub-micron) and heterogeneously mixed with primary nebular materials (e.g., Abreu and Brearley 2010). While the bulk elemental and isotopic compositions of CRs provide extremely valuable insights on how prevalent the changes 
observed at the nanometer scale, Abreu (2016a) suggested that several characteristics of the CRs limit their applicability as indicators of aqueous alteration. First, these measurements are affected by terrestrial weathering and impact processes (Alexander et al., 2013) - processes that have extensively affected many CRs (Abreu 2016a). Second, CRs contain variable abundance of clasts from different regions of the CR parent body or from altogether different planetary bodies (i.e., Hiyagon et al. 2016; Abreu 2013; MacPherson et al., 2009); consequently gram-size samples may contain numerous clasts recording diverse aqueous alteration histories. Third, intra- and inter-sample variations in CR matrix and dark inclusion abundances are too large to allow to resolve CR sub-groups based on bulk compositional and isotopic data alone (Abreu 2016). A multi-technique approach is recommended to measure the degree of aqueous alteration of CRs.

$\mathrm{CR}$ chondrites are not as water-rich as the CM or CI chondrites. However, members of the CR group have affinities with other groups of hydrated meteorites, including the CI (e.g., Bischoff et al., 1993a, b; Zolensky et al., 1993; Kallemeyn et al., 1994) and CM chondrites (e.g., Abreu 2016; Zolensky et al., 1993; Kallemeyn et al., 1994). Alteration of CIs resulted in the formation of phyllosilicates, oxides, carbonates, sulfides, and sulfates (Kerridge et al., 1979; Tomeoka et al., 1988; Zolensky et al., 1993). CRs, CIs and some CMs share the presence of framboidal and platelet magnetite. The mineralogy of some CR matrices also resembles assemblages in CM chondrite matrices (Abreu, 2016a). Alteration of CM matrices resulted in the formation of phyllosilicates, tochilinite, carbonates, sulfides, sulfates, oxides, and hydroxides (e.g., Browning et al., 1996; Chizmadia and Brearley, 2008; de Leuw et al., 2010; Hanowski and Brearley, 2001; Hewins et al., 2014; McSween Jr, 1979; Rubin et al., 2007; Tomeoka and Buseck, 1985; Zega and Buseck, 2003; Zolensky et al., 1993). Although CR chondrites share many similarities with CI and CM chondrites, the matrices of these meteorites contain variable amounts of amorphous Fe-Mg silicates (e.g. Abreu and Brearley, 2010; Le Guillou et al., 2015ab; Abreu, 2016a). Le Guillou et al. (2015a) used a combination of STXM and TEM data to determine the Fe-oxidation state of CR matrix silicates.

\subsection{Impact-Driven Thermal Metamorphism in CR Chondrites}

Most CR chondrites have largely escaped thermal metamorphism. Mild effects of heating have been consistently reported, including high-temperature reduction giving rise to low Co and $\mathrm{Ni}$ contents at the edges of $\mathrm{Fe}-\mathrm{Ni}$ metal grains (Lee et al. 1992) and redistribution of $\mathrm{Cr}_{2} \mathrm{O}_{3}$ in FeO-rich chondrule olivines (Schrader et al. 2015; Abreu 2016b). However, more significant heating has only been reported in two CR chondrites, GRA 06100 and GRO 03116 (Abreu and Bullock 2013; Briani et al. 2013; Schrader et al. 2015). All these reports suggest that the mechanism driving heating had short duration and was most like an impact event.

Abreu and Bullock (2013) argued that opaque assemblages in GRA 06100 formed through shock metamorphism followed by hydrothermal alteration of $\mathrm{Fe}-\mathrm{Ni}$ metal and $\mathrm{Fe}-(\mathrm{Ni})$ sulfides by fluids released during the impact event. Unlike metal nodules in other CR chondrites described above, opaques GRA 06100 are aggregates of exotic, high-temperature minerals. Ironnickel metal in GRA 06100 is rare and dominated by Ni-poor and Ni-rich intergrowths, in which Co appears to have preferentially partitioned into kamacite. As demonstrated by Kimura et al. (2008, 2011), such compositional trends are the result of thermal metamorphism. Kamacitetaenite nodules in GRA 06100 are invariably surrounded by an assortment of fine-grained $(<50$ micron) phases. FIB/TEM analysis of one of these regions revealed the presence of suessite, a 
diaplectic glass that has been found in shocked ureilites (e.g., Rubin 1988). Other accessory phases include Ti-Cr-Fe-Ni alloys, as well as the high-temperature hydrothermal phosphatesulfates scorzalite-lazulite and the end-member carbonate ankerite. The opaque mineral textures and presence of glass suggest that the heat source that resulted in metamorphic fractionation of $\mathrm{Ni}$ and $\mathrm{Co}$ in metal was probably an impact and that this event was probably followed by hydrothermal alteration at temperatures above those typical of other aqueously altered CR chondrites. Shock-driven metamorphism of GRA 06100 is supported by the presence oxidesulfide-metal lodes that not only trace the outlines of chondrules but also form networks that appear to connect opaque nodules and opaque assemblages. These textures suggest postaccretion mobilization of metal and sulfides, which is generally thought to arise from impact events. Furthermore, FIB/ TEM images show the presence of additional diaplectic glass in these regions. Finally, opaque assemblages that are similar in texture and mineralogy to the opaque assemblages formerly known as Fremdlinge are ubiquitous in GRA 06100. FE-EPMA analysis showed that these occurrences also have very similar chemical compositions to the Fremdlinge present in CAIs in CV chondrites (e.g., Blum et al. 1988, 1989). In fact, major, minor, and trace element siderophile element patterns spanning a broad range of volatilities show very similar trend. Although early studies of Fremdlinge in CV chondrites argued that these assemblages condensed in the solar nebula, it is generally accepted that they formed through a complex sequence of high temperature asteroidal processes. Such processes are consistent with other evidence observed in GRA 06100 opaques, further supporting the initial hypothesis that this meteorite was processed at temperatures that are potentially hundreds of degrees higher than other CR chondrites. As the matrix of GRA 06100 is an aggregate of amorphous silicates and phyllosilicates, observations of opaque component in GRA 06100 appear to be more sensitive to thermal metamorphism than petrologic changes in the matrix, making analysis of CR metal a valuable tool in understanding the history of these meteorites. Based on observations from GRA 06100, the CR parent body records at least an impact metamorphism event in which the initial shock heating resulted in the formation of Ni-poor and Ni-rich sub grains, mobilization of Fe from precursor Fe-Ni metal that created ephemeral shock veins, formation of suessite, and resulted in overall comminution and deformation of the meteorite. Subsequent aqueous alteration gave rise to the $\mathrm{Fe}$ sulfides and Fe oxides that dominate the mineralogy of opaque assemblages and cracks throughout GRA 06100. Exchange of shock metamorphic gases and fluids probably occurred at the centimeter-scale based on the characteristics of partly filled cracks and low abundance of sulfur in the adjacent matrix.

To constrain the degree of heating of CR chondrites, Briani et al. (2013) measured the Ni content of metal nodules, the hydration state of matrix, and structure and composition of organic matter in 15 CR: EET 87711, GRA 06100, GRA 95229, GRA 98025, GRO 03116, GRO 95577, LAP 02342, LAP 04516, LAP 04592, MAC 87320, MET 00426, PCA 91082, QUE 99177, Renazzo, and Sahara 00182. They concluded that only two of those CR chondrites showed signs of heating, GRA 06100 and GRO 03116, which is consistent with previous analyses of these two samples (Abreu, 2011; Abreu and Singletary, 2011). Using infrared spectra, Briani et al. (2013) found that, unlike other CR chondrite matrices, the matrices of both GRA 06100 and GRO 03116 shown significant signs of dehydration. The organic materials in these two meteorites were also distinct from that of other $\mathrm{CR}$ chondrites, presenting a comparatively low carbonyl abundance and high $\mathrm{CH}_{2} / \mathrm{CH}_{3}$ ratio (Briani et al. 2013). They argued that the thermal metamorphism style of the CR chondrites was different from other chondrite groups in which heating has been attributed to the decay of short-lived radioisotopes. 
Schrader et al. (2015) measure the major and minor element abundances of olivine, pyroxene, metal, and sulfide phases within a total of 210 chondrules in order to estimated the degree of thermal metamorphism for 15 unpaired CR chondrites, including some of the same samples analyzed by Briani et al. (2013): Al Rais, EET 87770, EET 96259, Gao-Guenie (b), GRA 95229, GRA 06100, GRO 03116, LAP 02342, LAP 04720, MET 00426, NWA 801 UA2300, PCA 91082, QUE) 99177, Shişr 033, and Yamato 793495. Based on the redistribution of $\mathrm{Cr} 2 \mathrm{O} 3$ in type II chondrule olivines, they found that least thermally altered CR chondrite is EET 96259 followed by QUE 99177. In contrast, they determined that the most thermally altered is Y-793495 followed by NWA 801, GRA 06100, and EET 87770. However, they cautioned that given the low numbers of type II chondrules in CR chondrites, some variations in the ordering might be possible.

Finally, Abreu (2016a) studied the composition of Fe-Ni metal, type I and II chondrule phenocrysts, and mesostasis of nine, unpaired, Antarctic CR chondrites: EET 92062, LAP 02342, GRA 95229, MIL 07525, EET 96259, MIL 090001, LAP 04516, LAP 04720, GRO 95577. This study excluded GRA 06100 and GRO 03116. The indicators of thermal metamorphism record contradictory trends in the CR chondrites studied by Abreu (2016a). According to these analyses, the distribution of $\mathrm{Ni}$ and $\mathrm{Co}$ in $\mathrm{Fe}-\mathrm{Ni}$ metal suggest that all the CRs have undergone metamorphism consistent with petrologic type $\sim 3.1$, which is generally consistent with results presented by Schrader et al. (2015). Therefore, if a relationship between thermal metamorphism and aqueous alteration exists (e.g., type IA chondrules), it does not appear that heating was a main driver in the formation of hydrated minerals. Therefore, all studies are consistent that most CR chondrites record only extremely mild thermal metamorphism and that when present; it is most likely attributable to collisional event(s).

\subsection{Regolith Processes Affecting CR Chondrites}

$\mathrm{CR}$ chondrites show evidence of mixing of materials formed in different locations of carbonaceous chondritic parent bodies or altogether different parent bodies. There are two types of foreign materials in CR chondrites. First, most CR chondrites contain numerous, matrix-like, extensively aqueously altered dark inclusions described above. The size and abundance of these dark inclusions is highly variable. In addition, two CR chondrites, QUE 99177 and NWA 801, contain extensively heated, possibly differentiated clasts that may have originated in planetarysized bodies (i.e., the size of the Moon).

\subsection{1 Chondritic Dark Inclusions CR Chondrites}

Dark inclusions (DIs) are fine-grained clasts up to $\mathrm{mm}$ in length (e.g. Scott et al. 1988). CR chondrites are characterized by a higher abundance of dark inclusions (DIs) compared to other chondritic meteorites (e.g. Bischoff et al. 1993a-b; Endre $\beta$ et al. 1994; Krot et al. 2002). Dark inclusions are larger and more abundant in GRO 95577 (Weisberg and Huber 2007; Tyra 2013).

In most weakly and moderately altered CRs, dark inclusions range from 0.7 to $3 \mathrm{~mm}$ along their maximum dimensions. They comprise up to $\sim 20$ vol. $\%$ of the fine-grained material in some CRs. Texturally, dark inclusions are distinct from the host meteorite with a significantly lower abundance of chondrules and finer grained matrix material in the host meteorites (Fig. 4c). Dark inclusions are made out of fine-grained silicates, Fe-oxides and sulfides, roughly in order of decreasing abundance. These clasts contain variable amounts of $\mu \mathrm{m}$-sized opaques, which 
generally occur as framboidal masses (up to $50 \mu \mathrm{m}$ in length - e.g. Fig. $4 d$ ) or rounded $(<20 \mu \mathrm{m})$ Fe-oxides, probably magnetite. Other textures include large, elongated, feathery, and filamentous. Opaques in some DIs are oriented.

The relationship between the host CR chondrites and dark inclusions has not been clearly established. Zolensky et al. (1993) suggested that despite the differences in texture and grain size, CR matrices and dark inclusions were genetically related. Zolensky et al. (1993) suggested that the matrix had originated from secondary processing of dark inclusion-material. On the other hand, Endreß et al. (1994) concluded that CR matrix and DIs bear no genetic relation, based on isotopic evidence, and may have been altered in completely different environments. In addition, Jilly-Rehak et al. (2018) observed that carbonates in interchondrule matrix and dark inclusions plot along a single array with slope $\sim 0.7$, which they interpreted as both lithologies recording alteration by a single evolving fluid on the CR parent body.

\subsection{2 Xenoliths from Planetary-sized Bodies in CR Chondrites}

Xenolith clasts that may have originated in planetary-sized bodies have been identified in two CR chondrites, QUE 99177 and NWA 801 (Abreu 2007; Abreu 2013; Kimura et al. 2013; Hiyagon et al. 2016). Clasts in these meteorites share some mineralogical characteristics, most prominently the presence of omphacite, which has not been identified in any other meteorite group (Abreu 2007; Abreu 2013; Kimura et al. 2013; Hiyagon et al. 2016). Dominant minerals in clasts in both meteorites are olivines and pyroxenes. Olivines in the three clasts identified in NWA 801 are more equilibrated and have slightly higher $\mathrm{MgO}$ contents compared to the clast in QUE 99177 (Fo59-64 and Fo average for QUE 99177 clast - Abreu 2013 versus Fo66-68 and Fo67.2 average for NWA 801 clasts - Kimura et al. 2013). Pyroxenes in the clasts in both meteorites have very similar compositions ( $\mathrm{En}_{74.0} \mathrm{Fs}_{24.4} \mathrm{Wo}_{1.5}$ average for QUE 99177 clast; Abreu 2013 and $\mathrm{En}_{73.2} \mathrm{Fs}_{26.1} \mathrm{Wo}_{0.7}$ average for NWA 801 clasts; Kimura et al. 2013). Clasts in both meteorites contain sulfides (pentlandite and troilite).

Clasts omphacites differ in crystal structure and chemical composition, suggesting that the clasts in QUE 99177 and NWA 801 had different formational histories. Crystallographically, an omphacite crystal in the QUE 9177 clast was indexed to the P2/n, whereas omphacites in the clasts in NWA 801 have C2/c structure. The P2/n polymorph (i.e., QUE 99177 clast) indicates high cation ordering, whereas the $\mathrm{C} 2 / \mathrm{c}$ (i.e., clasts in NWA 801) corresponds to a higher temperature, higher disordered structure (Fleet et al. 1978; McNamara 2012; Moghadam et al. 2010; Oberti and Caporuscio 1991). In slow exhumation, terrestrial tectonic settings, disordered C2/c transition to the P2/n polymorph (Fleet et al. 1978). Differences in crystallographic structure omphacites suggest that: omphacite in the QUE 99177 clast formed at a slower rate than in the NWA 801 clasts; (2) that these xenoliths were excavated at different rates (highly unlikely), or (3) that the clast in QUE 99177 underwent a later metamorphic event that is not recorded by the clasts in NWA 801. Hiyagon et al. (2016) reported that the composition of omphacite in the NWA801 clasts is more equilibrated, Na-rich, and Ca-poor compared with that in the QUE 99177 clast. Omphacites are slightly more ferroan in the NWA 801 clasts (i.e., 5.87.3 wt.\% FeO; Kimura et al. 2013) than in QUE 99177 clast (i.e., 5.2 wt.\% FeO; Abreu 2013) and higher in $\mathrm{Al}_{2} \mathrm{O}_{3}$ (7-9.4 wt.\% in QUE 99177 versus 6.6 wt.\% in NWA 801). Higher $\mathrm{Al}_{2} \mathrm{O}_{3}$ contents in omphacite could indicate a higher formational pressure for the clasts in NWA 801. If all other parameters are assumed to be equal, omphacite compositions would suggest that the clasts in NWA 801 would originate from a higher depth. Although both clasts contain graphite, 
crystals in the QUE 99177 clast are coarser-grained (up to $70 \mu \mathrm{m}$ ) and more abundant than in the clasts in NWA 801 (Abreu 2013; Kimura et al. 2013).

Despite mineralogical similarities, important textural and mineralogical differences exist between the clasts in QUE 99177 and NWA 801. For example, Abreu (2013) observed that the clasts in QUE 99177 consists of micron-sized, compositionally equilibrated matrix ( 48 vol\%) that has partially integrated with graphite-free, chondrule-like objects or chondrule relicts. The matrix in the QUE 99177 clast contains coarse, interlocking crystals. The chondrules are extensively recrystallized, contain no mesostasis or metal, and present poorly-defined boundaries. While Kimura et al. (2013) observed that $\sim 10$ vol.\% of clasts in NWA 801 were made up of finer-grained lithology (termed "graphite-bearing lithology") dominated by micronsized silicates and coarse-grained "graphite-free lithology", they reported that no chondrule relicts were identified. Although clasts in both meteorites contain hydrous phases, clast in QUE 99177 contains amphiboles (pargasite and pargasitic hornblende) where as the clasts in NWA 801 have small amounts of the phyllosilicate phlogopite. Unlike the clast in QUE 99177, the clasts in NWA 801 contains garnets with large pyrope and almandine components (Kimura et al. 2013). Coexisting omphacite and garnets indicate that NWA 801 clasts forned in an eclogite-like environment (Kimura et al. 2013). The accessory-phase assemblages are also distict for the clasts these two CR chondrites. Clasts in NWA 801 contain chloroapatite and metallic Fe, which were not identified in the clast in QUE 99177 (Abreu 2013; Kimura et al. 2013; Hiyagon et al. 2016).

Calculations of minimal overburden pressures for clasts in these meteorites were performed very different methods, and perhaps unsurprisingly, had very different results. Kimura et al. (2013) were able to use terrestrial geothermobarometers based on the fact clasts NWA 801 have coexisting olivine, pyroxenes, and garnet (garnet is not present in the clast in QUE 99177). They estimated that $2.8-4.2 \mathrm{GPa}$ and $940-1080^{\circ} \mathrm{C}$ were required to form the clasts in NWA 801. In contrast, the compositional and crystallographic features of the QUE 99177 clast indicate that omphacite formed at pressures in the excess of $>6 \mathrm{GPa}$ (Abreu 2013). This pressure corresponds to an overburden depth of $\sim 760 \mathrm{~km}$ if typical B-type asteroid densities are assumed.

Studies of the clasts in QUE 99177 and NWA 801 coincide in that theit high-pressure phases must have formed over long periods of time (i.e., not during impact events) by overburden in planetary-sized, largely undifferentiated bodies that were later catastrophically disrupted (Abreu 2013; Kimura et al. 2013; Hiyagon et al. 2016). In fact, Hiyagon et al. (2016) estimated that clasts in NWA 801 formed over timescales of the order of $10^{2}-10^{3}$ years, based on the diffusion rates for $\mathrm{Mg}-\mathrm{Fe}$ in olivine and orthopyroxene at a temperature of $1000^{\circ} \mathrm{C}$. However, two very different formational histories have been proposed for omphacite-bearing clasts in QUE 99177 and NWA 801 (Abreu 2013; Kimura et al. 2013; Hiyagon et al. 2016). Abreu (2013) proposed that all mineral phases in the QUE 99177 clast formed and experienced transitions in the solid state during multiple heating events. In constrast, Hiyagon et al. (2016) argued that clasts in NWA 801 were generated by localized igneous processes.

Based on the presence of partially-integrated relic chondrules and matrix, Abreu (2013) favored a metamorphic origin in a large asteroid that was catastrophically disrupted at least once and which material re-accreted in the CR-forming region. According to Abreu (2013), omphacite and graphite formation were the first steps in the QUE 99177 formation sequence. The high abundance of carbon in the omphacite clast could have resulted from graphitization of a carbonrich precursor, such as a C-rich carbonaceous chondrite. Amphibole formation postdated graphite formation and occurred by reaction of olivine, clinopyroxene, and omphacite in the presence of an oxidizing, Na-rich fluid at high temperatures $\left(>700^{\circ} \mathrm{C}\right)$. Even if omphacites in 
the clast in QUE 99177 originally formed at high-temperature, a later high-temperature amphibole-forming event could have been sufficient for an order-disorder transition into the P2/n polymorph to occur. The absence of metal suggests that this fluid was also rich in $\mathrm{S}$. It is possible that amphibole formation may have occurred at initially high temperature $\left(\sim 760^{\circ} \mathrm{C}\right)$ and that sulfide $\left(<650^{\circ} \mathrm{C}\right)$ and graphite $\left(>550^{\circ} \mathrm{C}\right)$ formation occurred as temperature decreased. Fluid evolution is likely to have played a central role in the final mineralogy. Although the conditions under which the clast formed were reducing, interaction with strongly oxidizing fluids may have resulted in the formation of $\mathrm{Fe}^{3+}$-bearing amphiboles. Further evolution into an $\mathrm{H}_{2} \mathrm{~S}$-rich fluid may have been very effective in sulfidizing pre-existing Fe-Ni alloys. Abreu argued that the omphacite-bearing clast in QUE 99177 must have formed in the interior of a large, possibly ephemeral asteroid and was excavated by a large impact. Formation of omphacite and retention of carbon and sulfur during high-temperature metamorphism are indicative of formation under high-pressure.

Hiyagon et al. (2016) outlined a very different sequence of events for clasts in NWA 801. Hiyagon et al. (2016) suggested that the presence/absence of graphite in the otherwise similar lithologies in NWA 801 clasts was due to formation of the different lithologies at different depths. In this model, the two lithologies where mixed, buried, and sintered over multiple collisional events. A subsequent large collision would have given rise to a Moon-size planetary object and the clasts in NWA 801 clasts would be emplaced near the center of this ephemeral planet. In these location the following processes would have taken place over $10^{2}-10^{3}$ years: of the eclogitic phases (i.e., omphacite and garnet), homogenization of Fe-Mg olivine and pyroxenes, and redistribution of P and REEs between two lithologies (Hiyagon et al. 2016). Finally a collision would have catastrophically disrupted the Moon-sized body, quenching the clasts and transporting them to the region in which they accreated to form the CR parent body. The origin of the single hydrous phase, phlogopite was not discussed in detail within this model.

It is unclear if clasts in QUE 99177 and NWA 801 had different precursors and/or different histories. Their different mineralogical assemblages, in particular the high abundance of graphite and absence of garnet in the clast in QUE 99177 suggest that the precursor's composition was indeed difference (e.g., Hiyagon et al 2016). More detailed comparative studies are needed to determine if these clasts had indeed as different histories as current investigations suggest (Abreu 2013; Kimura et al. 2013; Hiyagon et al. 2016). It is important to note that the graphite-free lithology in NWA 801 clasts (Kimura et al. 2013) closely resembles the relict chondriltes that Abreu (2013) identified in the QUE 99177 clast. The graphite-bearing lithology in NWA 801 clasts also resemble the matrix materials in the QUE 99177 clast. The absence of graphite in relict chondrules in the QUE 99177 clast is easily explained by the fact that chondrule precursors would have been essentially carbon free (Abreu 2013). In contrast, formation of the graphite-bearing and the graphite-free lithologies at different depths would require a much more complex sequence of excavation events Hiyagon et al. (2016).

\subsection{Terrestrial Chemical and Mineralogical Processes Affecting the CR Chondrites}

\subsection{Terrestrial Non-Silicates in CR Chondrites}

The dominant effects that terrestrial weathering have on meteorites, Fe oxidation and mobilization, may affect CR chondrites more extensively than other hydrated meteorites because the CRs are comparatively rich in $\mathrm{Fe}-\mathrm{Ni}$ metal. Terrestrial weathering has extensively affected $\mathrm{Fe}-\mathrm{Ni}$ metal and Fe-oxides. Most Fe-Ni metal grains are located inside the chondrules or on the 
margins of chondrules adjacent to fine-grained matrix. These nodules reach up to several hundreds of microns in diameter.

Abreu (2016) observed that there are no systematic differences between the weathering features in Fe-Ni metal grains located in chondrule interiors versus those in direct contact with matrix. Abreu (2016) described that the effect of Antarctic weathering on CR Fe-Ni metal included thick rust rims, oxide sub-grains, and even complete pseudomorphic replacement in some cases. Fe-hydroxide veins are commonly observed on the periphery of Fe-Ni metal nodules. In a CR graded at the most advanced degree of elemental mobilization and mineral replacement (LAP 04720, grade C), Fe-hydroxide veins can cut across the length of thin section.

Le Guillou et al. (2015) suggested that some pure ferric Fe phases, such as hematite and ferrihydrite, which are generally assumed to be terrestrial, may in fact have formed as matrix silicates were progressively altered and their $\mathrm{Fe}^{3+} / \sum \mathrm{Fe}$ ratios decreased. However, it is unclear if and how much hematite and ferrihydrite are present in the most altered CR find, Al Rais. If significant amounts of these ferric Fe minerals are present in Al Rais, the origin of ferric Fe minerals in Antarctic chondrites ought to be revisited.

\subsection{Terrestrial Silicates in CR Chondrites}

The effect of terrestrial alteration on the silicate mineralogy of CR chondrites remains largely unexplored, in particular for samples from hot deserts. Abreu (2016) observed that in weathering grade B/C MIL 07525, silicate matrix regions adjacent to altered metal are unusually Fe-rich. One of such regions located in MIL 07525 was investigated in detail (Fig. 1). Amorphous Fe-Mg silicates in this CR contain $\sim 17 \%$ more $\mathrm{FeO}$ and than similar materials in regions that did not show textural evidence of terrestrial weathering. Amorphous Fe-Mg silicates were associated with the ferroan serpentine and hisingerite ( $30 \AA$ basal spacing). However, more in-depth studies are necessary because the effect of terrestrial weathering of CR silicates is not easily resolved. Terrestrial alteration products, in particular those formed under Antarctic conditions, may resemble aqueous alteration occurring at the low temperature, low water:rock ratios thought to be prevalent in the CR parent body.

\subsection{Implications for Exploration of CR Parent Bodies.}

Exploration of CR parent bodies holds great promise for both science and in-situ resource utilization (ISRU). A sample-return mission to a CR parent body, such as the one that had been planned for ARRM has the potential of becoming the equivalent of the Beagle expedition in terms of understanding the evolution of our solar system. Scientifically, we have ample evidence that CR chondrites carry a fundamental record of both astrophysical and geological processes. Exploration of CR parent bodies has the potential to shed light on the earliest processes occurring in the solar system and beyond. CR chondrites contain a myriad of presolar materials of very diverse provenance, which description is beyond the scope of this work. In addition, CR chondrites are rich in exotic organic matter, which may have had a highly diverse history (e.g., Glavin et al. this volume). The mineralogy of CR chondrites has revealed that most of these meteorites have largely escaped aqueous alteration and thermal metamorphism and represent in many ways a " $t_{0}$ " time capsule for the formation of terrestrial planets. In addition to this highly pristine materials, CR chondrites host exotic clasts of objects that no longer exist in the solar system. Furthermore, the mineralogical characteristics of CR chondrites have the potential to facilitate extraction of both water and organics, which are distributed at the nanometer scale within abundant metastable, amorphous, Fe-Mg silicates. 
Petrologic studies of CR chondrites can address the ISRU-relevant questions, including: (i) How are Fe-Ni metal nodules affected by aqueous and hydrothermal alteration in asteroids?; (ii) What is the distribution of water-bearing minerals in heated and unheated CR-like materials?; and (iii) What is the effect of thermal metamorphism on the distribution of organics? Several studies proposed the use of $\mathrm{H}_{2}$ and $\mathrm{O}_{2}$ from water-bearing minerals for in-situ production of fuel and propellants (e.g., Anand et al. 2012). In CRs, the mineralogical hosts of $\mathrm{OH}$ and $\mathrm{H}_{2} \mathrm{O}$ are amorphous Fe-Mg silicates and phyllosilicates. We are only beginning to characterize these materials. Understanding the effect of asteroidal processes on Fe-Ni metal will constrain the availability, distribution, and mineral host of precious metals for ISRU demonstrations. Extraction of volatiles and metals will involve material processing: removing and mechanically processing the regolith (e.g., drilling, scooping, crushing, sorting, etc.) and chemically processing the regolith (e.g., heat of volatilization, reaction mechanisms). Designing tools to accomplish ISRU goals should be informed by the distribution, chemical composition, and mineralogical characteristics of analog materials.

Spectral matches between CRs and potential parent bodies are hindered by the effect of terrestrial weathering on the mineralogy of these meteorites. We face multiple challenges to address this uncertainty. First, it is not clear what the characteristics of un-weathered CRs are, because only Renazzo and anomalous Al Rais are observed falls. All other CRs are finds, which are more prone to chemical interaction with the terrestrial environment. The fact that $\mathrm{CR}$ chondrites contain both large volumes of fine-grained metallic Fe and sub-micron amorphous silicates aggravates the effects of terrestrial weathering on these meteorites. Second, terrestrial alteration and asteroidal alteration products resemble each other. Third, the effect of terrestrial weathering on amorphous Fe-Mg-rich silicates has not been studied. Fourth, because CR chondrite silicates are generally Fe-poor, they have weak Fe-silicate-associated absorption bands, making them prone to overprinting by terrestrial weathering products. Therefore, detailed studies of the effects of terrestrial processes on CR chondrites need to be conducted.

\subsubsection{Chemical Composition and Mineralogy of CO Chondrites and Processes Affecting these Meteorites}

\subsubsection{Physical Properties of $\mathrm{CO}$ chondrites}

Macke et al. (2011) examined the bulk porosity of two Ornans-like chondrites, Isna (4\%) and Lancé. (8.3\%). Macke et al. (2011) measured the physical properties of $14 \mathrm{CO}$ chondrites (Colony, Dar al Gani 005, Dar al Gani 023, Dar al Gani 078, Dar al Gani 749, Felix, Isna, Kainsaz, Lancé, Moss, NWA 062, Ornans, Rainbow, and Warrenton), including multiple samples of each meteorite. They reported that the average (by meteorite) characteristics of $\mathrm{CO}$ chondrites are as follows, bulk density is $\sim 3.06 \mathrm{~g} / \mathrm{cm}^{3}$, grain density is $3.48 \mathrm{~g} / \mathrm{cm}^{3}$, and porosity is $11.6 \%$ (Macke et al., 2011). There is a large discrepancy between the porosity of Isna reported in these two studies (4\% versus 14.5\%). Macke et al. (2011) also noted that there was a significant difference between in the porosity of falls (average $\sim 19.4 \%$ ) versus finds (average $5.7 \%$ ). Smaller, yet discernable differences are described for the other physical properties. Rochette et al. (2008) also analyzed CO chondrites (28 in total) including falls, cold and hot desert finds. They found that, despite having abundant Fe-metal, $\mathrm{CO}$ chondrites have the lowest magnetization of all carbonaceous chondrite groups, $\log \chi=4.54 \pm 0.20$ and $4.49 \pm 0.28$, for falls and finds, respectively (in $10^{-9} \mathrm{~m}^{3} / \mathrm{kg}$ ). The values for the $\log \chi$ range from 4.99 from 5.99 , which they attributed to terrestrial weathering and metamorphism, with magnetic susceptibility 
decreasing with increasing thermal metamorphism. Macke et al. (2011) also measured the magnetization of $\mathrm{CO}$ chondrites, their values are generally consistent with those obtained by Rochette et al. (2008), $\log \chi=4.48\left(\log \chi *\right.$ in $\left.\log 10^{-9} \mathrm{~m}^{3} / \mathrm{kg}\right)$. Finally, Ibrahim (2012) determined the mean compressional wave velocity for CO chondrites to be $1526 \pm 374 \mathrm{~m} / \mathrm{s}$ and the average shear wave velocities equal $1526 \pm 374 \mathrm{~m} / \mathrm{s}$. They found the porosity and velocities are anticorrelated.

\subsubsection{Primary (Nebular) Characteristics of the CO Chondrites}

CO chondrites were initially identified as a distinct group of chondrites based on their bulk chemical composition and petrologic characteristics (e.g., McSween, 1977b; Kallemeyn and Wasson, 1981; Ikeda 1982; Rubin et al., 1985; Rubin 1998). Based on Position Sensitive Detector X-ray Diffraction of 11 low petrologic type Antarctic CO chondrites, Howard et al. (2014) reported that the bulk mineralogy of these meteorites consist of (vol.\%): total olivine (29$50 \%)$; pyroxene (10-24\%); feldspar (0-1.5\%) magnetite (5.5- 8.2\%); sulfide (4.7-6.7\%); metal (0.2-1.0\%); phyllosilicate (0-3.3\%); Fe-bearing amorphous (23-37\%). This group includes some of the most pristine meteorites, which record numerous solar nebula features (e.g., Brearley 1993; Nittler et al. 2013; Davidson et al. 2014b; Alexander et al., 2018).

\subsection{Chondrules in CO Chondrites:}

Like CM chondrules, chondrules in $\mathrm{CO}$ chondrites are characterized by their small diameters. Rubin (1989) measured the diameter of 2834 chondrules located in 11 CO chondrites obtained a mean value of 148 microns. Early reports indicated that chondrules occupied approximately $35-45 \%$ of the volume of these meteorites. However, more recent detailed measurements show that chondrule average volumes are in average $51.3 \%$, ranging from $43.35 \%$ in Ornans to $58.15 \%$ in Warrenton (Ebel et al. 2016).

Multiple suthors have noted that $\mathrm{CO}$ chondrules resemble those in ordinary chondrites albeit smaller in diameter. Chondrules are predominantly type I, MgO-rich, with fewer type II FeO-rich chondrules also represented (McSween, 1977b; Kallemeyn and Wasson, 1981; Rubin et al. 1985; Scott and Jones, 1990; Russell et al. 1998; Tomeoka and Itoh, 2004; Wick 2010). However, Berlin et al. (2011) noted that there was no compositional gap between these two groups of chondrules; in Kainsaz, the compositions of olivine phenocrysts in type I chondrules extend to $\mathrm{Fa}>5$ mole $\%$ and into the type II chondrule compositional regime.

Berlin also established that $\mathrm{CO}$ type II chondrules have lower $\mathrm{MnO}, \mathrm{Cr}_{2} \mathrm{O}_{3}$, and $\mathrm{CaO}$ than their counterparts in CR to UOC chondrites.

According to a recent review by Scott and Krot (2014), 95\% of CO chondrules are predominantly porphyritic. This textural variety includes POP (69\%), PP (18\%), and PO (8\%) chondrules. Non-porphyritic types include $2 \%$ barred olivine (BO), $2 \%$ radial pyroxene (RP), and $2 \%$ cryptocrystalline chondrules.

\section{Opaques in Chondrules in CO Chondrites}

Type I chondrules contain kamacite and taenite metal nodules, accounting for $1.8 \pm 1.05$ vol.\% of CO chondrites (McSween, 1977b; Kimura et al., 2008; Ebel et al. 2016). McSween (1977b) observed that taenite grains have Ni-rich rims and kamacite has Ni-poor rims. Scott and Jones (1990) measured the $\mathrm{Ni}$ and Co contents of kamacite grains to range from 3.4-6.2 wt.\% and $0.2-1.7 \mathrm{wt} . \%$ respectively. 


\section{Mesostasis in Chondrules in CO Chondrites}

In thermally metamorphosed $\mathrm{CO}$ chondrules, the mesostasis has been partially to fully recrystallized. Tomeoka and Itoh (2004) studied the mesostasis of 783 type I chondrules from seven $\mathrm{CO} 3$, ranging from petrologic type 3.0-3.7. They found that weakly heated mesostases are made up of glass and plagioclases, while more thermally processed samples contained nepheline. They suggested that nepheline formation occurred in two stages, beginning with a hydrous nepheline phase in the presence of aqueous fluids which was subsequently dehydrated. Berlin et al. (2011) examined the mesostasis of 6 type II chondrules in Kainsaz. They found that these regions of mesostasis were glassy, also containing crystallites. Compositionally, type II CO chondrule mesostasis was significantly more $\mathrm{Al}_{2} \mathrm{O}_{3}$-rich and had lower $\mathrm{SiO}_{2}$, low $\mathrm{TiO}_{2}, \mathrm{P}_{2} \mathrm{O}_{5}$, and $\mathrm{S}$ content than mesostasis in $\mathrm{CR}$ and UOC chondrules.

\subsection{Fine-Grained Materials in CO Chondrites}

Matrix and fine-grained clasts account for $40.1 \pm 6.04 \mathrm{vol} . \%$ of CO chondrites (Ebel et al. 2016). Early studies of CO fine-grained materials determined that they were dominated by finegrained olivines (Michel-Levy 1969; Kurat 1973, 1975). These olivines are generally finegrained $(<300 \mathrm{~nm})$ and variable in composition. Keller and Buseck (1990a) measured the compositional range to be Fa20-50 for Lancé. Brearley (1993) identified multiple populations based on composition and textures in ALH 77307: (1) a population with a range to be Fa 18-72, (2) a forsteritic population with a range to be Fo95-98, (3) clusters of fayalitic olivines with Fa66-88, and (4) Low-iron, manganese-enriched (LIME) olivines. McAdam et al. (2018) showed that amorphous Fe-Mg silicates are a main component of $\mathrm{CO}$ fine-grained materials.

\section{Fine-Grained Matrix in CO Chondrites}

TEM observations of Lancé confirmed that matrix is mainly comprised of small $(<0.1-10$ microns across), fragmental grains of olivine ( $\left.\sim \mathrm{Fa}_{45}\right)$, with numerous crystallographic defects (Keller and Buseck 1990a). They also revealed that these olivine grains are mixed with variable amounts of phyllosilicate, poorly crystalline silicate material, and minor Ca-rich and Ca-poor clinopyroxene, metal, and oxides (Keller and Buseck 1990a). The composition of Lancé matrix span a linear array perpendicular to the $\mathrm{MgO}-\mathrm{SiO}_{2}$ axis, showing variable, yet generally $\mathrm{FeO}$-rich compositions. The matrices of more thermally processed Kainsaz had a very different mineral assemblage, dominated by fine-grained olivine $\left(\mathrm{Fa}_{20-50}\right)$, enstatite, hedenbergite, and minor kamacity and taenite grains coated with crystalline graphite. Olivines in the matrix of Warrenton were more equilibrated (Keller and Buseck 1990a). Brearley (1993) examined a more pristine CO chondrite, ALH 77307 and corroborated that pristine CO matrix is indeed very rich in amorphous Fe-Mg-rich silicates. In addition, Brearley (1993) identified andradite, pyrrhotite, anhydrite, and mixed layer phyllosilicate phases. Compositionally, ALH 77307 matrix has low oxide totals, 89-91 wt \%, which was attributed to high density of fractures driving up the porosity. The average major element composition of ALH 77307 matrix was similar to the of Lancé matrix. The major elements in amorphous $\mathrm{Fe}-\mathrm{Mg}$-rich silicates was dominated by $\mathrm{FeO}-$ $\mathrm{MgO}-\mathrm{SiO}_{2}$; however, significant concentrations (1-8 wt \%) of of $\mathrm{Al}_{2} \mathrm{O}_{3}, \mathrm{NiO}, \mathrm{S}$ were detected. The origin of this matrix assemblage was proposed to result from disequilibrium condensation from a nebular gas. Noguchi et al. (1999) identified as similar matrix assemblage in Colony.

\section{Fine-Grained Rims in CO Chondrites}


Brearley (1993) examined fine-grained rims around chondrules in pristine ALH 77307. These rims ranged in thickness from 10-100 microns and often had cracks radiating from the chondrules. Chondrules with rims show no signs of brecciation. The major element composition of ALH 77307 rims was similar to that of matrix - albeit showing a narrower range of $\mathrm{FeO}$ contents. Like the matrix of ALH 77307, rims were dominated by amorphous Fe-Mg-rich silicates and fine-grained olivine grains.

\section{Dark Inclusions in CO Chondrites}

Itoh and Tomeoka (2003) estimated that dark inclusions represent approximately 1 vol.\% of CO chondrites. Itoh and Tomeoka (2000, 2003) studied in Lancé, Ornans, Lancé, and Warrenton. They described them as aggregates of fine grained (1-20 $\mu \mathrm{m})$, Fe-rich olivine (Fa4080) and minor diopside, Fe-Ni metal (kamacite and taenite), Fe sulfides, nepheline and $\mathrm{Ca}$ phosphate. Based on texture and mineralogy, Itoh and Tomeoka (2000) identified two types of dark inclusions. Type I dark inclusions $(10-40 \mu \mathrm{m})$ contained stacks of thin lath-shaped grains (1-3 $\mu \mathrm{m}$ in thickness and 10-15 $\mu \mathrm{m}$ in length) of Fe-poor olivine (Fa5-25) and resembled Allende. Type I dark inclusions were described as similar to dark inclusions in CV chondrites, containing elongated olivines (Itoh and Tomeoka, 2003). Despite the textural differences, Itoh and Tomeoka (2003) found no compositional differences between these two groups of dark inclusions. Itoh and Tomeoka (2003) suggested that CO dark inclusions formed by aqueous alteration and subsequence dehydration of a CO-like precursor. However, alteration is suggested to occur in a different location and that final emplacement occur prior to thermal metamorphism. They also suggested that type I dark inclusions represent fragments of chondrule pseudomorphs (Itoh and Tomeoka, 2003).

\subsection{Refractory Inclusions in CO Chondrites}

\section{CAIs in CO Chondrites}

Although they are not as abundant as in CV chondrites (3.04 \pm 2.00 vol.\%), CAIs still represent a significant fraction of $\mathrm{CO}$ chondrites, $2.3 \pm 0.23 \mathrm{vol} \%$ (Ebel et al. 2016). These CAIs have been studied in considerable detail (e.g., Fahey et al. 1994; Holmberg and Hashimoto 1992; Tomeoka et al. 1992; Kojima et al. 1995; Russell et al. 1998; Wasson et al. 2001; Itoh et al. 2004; Kunihiro et al. 2004; Kurahashi et al. 2008; Han and Brearley, 2016). Melilite-rich, spine-pyroxene, and hibonite-rich CAIs have been identified in CO chondrites.

\section{AOA in $\mathrm{CO}$ Chondrites}

AOAs represent $3.5 \pm 1.15$ vol.\% of CO chondrites (Ebel et al. 2016). Chizmadia et al. (2002) reported that CO AOAs were irregularly shaped and vary widely in size from 20 microns to $\sim 1 \mathrm{~mm}$. In the most pristine COs, AOAs contained $\sim 64$ vol. $\%$ olivine, $\sim 17$ vol. $\%$ anorthitic plagioclase, $\sim 12$ vol. $\%$ diopside, and $\sim 8$ vol. $\%$ opaques (Chizmadia et al. 2002). Anothite and diopside were generally located in the interior, whereas olivine was around them. In more thermally processed COs, Chizmadia et al. (2002) reported that AOAs had different proportions of these minerals: $\sim 67$ vol.\% olivine, $\sim 19$ vol. $\%$ anorthitic plagioclase, $\sim 14$ vol. $\%$ diopside, and $\sim 1$ vol. $\%$ opaques. The fayalite content of olivine was variable and correlated with the degree of thermal metamorphism. 


\subsection{Asteroidal Minerals:}

\subsection{Sulfur-bearing Phases in CO Chondrites}

Brearley (1993) reported nanophase pyrrhotite and pentlandites in the matrix of ALH 77307. Noguchi et al. (1999) also found troilite in Colony. These sulfides probably originated from the solar nebula. In addition, anhydrite was also identified in this meteorite and attributed to aqueous alteration.

\subsection{1 $\mathrm{Fe}$-Oxides in $\mathrm{CO}$ Chondrites}

Keller and Buseck (1990a) attributed the presence of poorly crystalline, Ni-bearing, $\mathrm{Fe}^{3+}$ oxide in the matrix of Lancé to replacement of matrix Fe-metal during aqueous alteration. In addition to these grains, Keller and Buseck (1990a) interpreted thin (1-2 nm) layers on matrix olivine surfaces and 100-nm thick layers on the sides of veins to the presence of $\mathrm{Fe}^{3+}$ oxide coatings. Brearley (1993) also reported fine-grained (<10 micron) magnetite in ALH 77307. Since Fe-metal was also present in the matrix of this meteorite, the presence of magnetite was also attributed to aqueous alteration of the metal. Finally, Noguchi et al. (1999) examined isolated, subhedral magnetite grains in the matrix of Colony. They found that these oxides have a small amount of $\mathrm{Cr}\left(<1 \mathrm{wt} \% \mathrm{Cr}_{2} \mathrm{O}_{3}\right)$, but are devoid of $\mathrm{Al}$, Ti, and $\mathrm{Mn}$.

\subsection{Fe-Carbides in CO Chondrites}

Scott and Jones (1990) reported the presence of fine-grained ( $<30$ microns) haxonite and cohenite in ALH A77307. Haxonite contained 5.0-5.7\% Ni and cohenite 2-4\% Ni; both contain $0.2-0.4 \%$ Co and up to $0.9 \% \mathrm{Cr}, 0.1 \% \mathrm{Si}$, and $0.2 \% \mathrm{P}$ (Scott and Jones, 1990). They noted that it was unclear if these carbides formed in the solar nebula or the asteroidal parent body. Later studies of carbides in OC chondrites suggest that these minerals might have formed in a parent body (Krot et al. 1997).

\subsection{Phyllosilicates in CO Chondrite Matrices}

Keller and Buseck (1990a) described the matrix of Lancé as an assemblage of Fe-bearing serpentine packets separated from the olivine surfaces by $\mathrm{Fe}^{3+}$ oxide. They estimated the composition of this serpentines to be $\left(\mathrm{Mg}_{2.2} \mathrm{Fe}_{0.8}\right) \mathrm{Si}_{2} \mathrm{O}_{5}(\mathrm{OH})_{4}$, In contrast, they found no phyllosilicates in thermally metamorphosed Kainsaz and Warrenton. Brearley (1993) identified coarse-grained (0.3-1 micron) phyllosilicates in association with amorphous Fe-Mg silicates in the matrix of ALH 77307. These layered minerals had basal spacing including 0.7, 0.85, 0.96, 1.08 , and $1.2 \mathrm{~nm}$, which were matched to serpentine, tochilinite, and smectite. Noguchi et al. (1999) also identified saponite, serpentine, chlorite, and montomorillonite in the matrix of Colony.

\subsection{Asteroidal Chemical and Mineralogical Processes Affecting the CO Chondrites}

\subsection{Models of Aqueous Alteration Processes Affecting the CO Chondrites}

$\mathrm{CO}$ chondrites are thought to have undergone hydrothermal aqueous alteration to a lesser degree than $\mathrm{CV}$ chondrites. The effect of metasomatism has been studied in both CAIs and chondrules. Zolensky et al. (1993) suggested that the CO chondrites experienced parent body 
conditions of low $f_{\mathrm{O} 2}$, low water/rock ratios, and temperatures below $50^{\circ} \mathrm{C}$. CO CAIs record asteroidal metasomatism, including melilite replacement (e.g., Russell et al. 1998; Wasson et al. 2001; Itoh et al. 2004). Tomeoka and Itoh (2004) suggested that the replacement of mesostasis of type I CO chondrules records two metasomatic processes: (1) Na-metasomatism represented by the formation of Na-rich nepheline in mesostasis and (2) and Fe-metasomatism represented by the Fe-enrichment in olivine and pyroxene phenocrysts. They suggested that the $\mathrm{CO}$ chondrites record at least three different asteroidal events: (1) low-grade aqueous alteration; (2) hightemperature dehydration; and (3) thermal diffusive exchange of atoms without the assistance of fluids. Oxygen isotopic studies support the suggestions that the thermal events affecting the $\mathrm{CO}$ chondrites involved an aqueous fluid (Greenwood and Franchi 2004).

\subsection{Thermal and Hydrothermal Metamorphism in CO Chondrites}

Despite the primitive features of some CO chondrites, McSween (1977b) recognized that some $\mathrm{CO}$ chondrites have been affected by thermal metamorphism and ordered the meteorites he examined in the following sequence of increasing grade: Kainsaz, Felix, Ornans, Lancé, Isna, and Warrenton. In this sequence the Mg-number of chondrule silicates progressively decreased, the chemical composition of Fe-Ni metal nodules changed (Ni and Co contents in kamacite increased and the $\mathrm{Cr}$ content decreased with metamorphism), and matrix and chondrules showed signs of textural and compositional integration. Since the publication of the seminal McSween (1977b) paper, a number of different schemes have been proposed to determined to what degree thermal metamorphism has affected the CO chondrites (e.g., Scott and Jones 1990; Grossman and Brearley 2005; Sears et al. 1991; Bonal et al. 2007; Kimura et al. 2008). Some studies focused on the oxide content of chondrule phenocrysts, namely $\mathrm{FeO}$ contents (e.g., Scott and Jones 1990) and $\mathrm{Cr}_{2} \mathrm{O}_{3}$ contents in type II chondrule phenocrysts (Grossman and Brearley 2005). Co-Ni systematics has been used to measured their degree of thermal metamorphism (Scott and Jones, 1990; Kimura et al. 2008). Scott and Jones (1990) also noted the there was a trend in which Co contents of kamacite became more homogeneous with thermal metamorphism. Chizmadia et al. (2002) argued that AOAs are sensitive indicators of thermal metamorphism, because of their fine-grained nature. Chizmadia et al. (2002) refined the classification based on the characteristics of AOAs, namely the fayalite content of olivine, which they found increases from $\mathrm{Fa}_{0-1}$ in type 3.0 to Fa60-75 in type 3.8 CO chondrules. Tomeoka and Itoh (2004) used the changes in the chemical composition of type I CO chondrule mesostasis to assess to what degree they had been affected by thermal metamorphism. Instead of focusing on mineralogy and petrology changes alone, Bonal et al. (2007) used Raman spectroscopy to examine the maturation grade of the insoluble organic matter in CO matrices and assign petrologic sub-types to these samples. They assigned ALHA77307 to type 3.03, Colony to 3.1; Kainsaz, Felix, Lancé, and Ornans to 3.6, and Warrenton and Isna to type 3.7.

\subsection{Terrestrial Chemical and Mineralogical Processes Affecting the CO Chondrites}

\subsection{Terrestrial Non-Silicates in CO Chondrites}


Studies of the style of terrestrial weathering of $\mathrm{CO}$ chondrites remain limited (e.g., Noguchi et al., 1999), as the most extensively studied CO chondrites are falls. In addition to these poorly-crystalline Fe-oxides, Keller and Buseck (1990a) identified well-crystallized ferrihydrite $\left(5 \mathrm{Fe}_{2} \mathrm{O}_{3}+9 \mathrm{H}_{2} \mathrm{O}\right)$ in Lancé. Although Lancé is a fall, they did not rule out the possibility that these oxyhydroxides could be terrestrial.

\subsection{Terrestrial Silicates in CO Chondrites}

Noguchi et al. (1999) reported the presence of goethite and of limonite veins in the matrix of extensively weathered Colony. Although they attributed the formation of amorphous silicates in the matrix of Colony to terrestrial processing, more recent studies of primitive $\mathrm{CO}$ chondrites suggest that at least some of these materials may be nebular in origin.

\subsubsection{Physical Properties, Chemical Composition, and Mineralogy of CV Chondrites and Processes Affecting these Meteorites}

\subsubsection{Physical Properties of CV chondrites}

Corrigan et al. (1997) measured the porosity for seven CV chondrites (oxidized $\mathrm{CV}_{\mathrm{os}}$ : Allende, Bali, Kaba, and Mokoia; and reduced $\mathrm{CV}_{\mathrm{rs}}$ : Efremovka, Leoville, and Vigarano description of these sub-groups is given below). Allende and Mokoia matrices had high porosities (25\% and 30\%, respectively), whereas the porosities of Bali, Efremovka, Kaba, and Vigarano matrices were very low (1-6\%) (Corrigan et al., 1997). There was also a similar, yet smaller gap between the bulk porosities of Allende and Mokoia (20\% and 24\%) and the other meteorites ( 2\%-10\%), including multiple Vigarano lithologies (ranged in porosity from 27\%). Macke et al. (2011) measured the porosity of 75 stones of $12 \mathrm{CV}$ chondrites, including 52 stones from Allende (oxidized $\mathrm{CV}_{\mathrm{o}}$ : Allende, Axtell, Grosnaja, Mokoia, and Nova 002; reduced $\mathrm{CV}_{\mathrm{rs}}$ : Efremovka, Leoville, and Vigarano; Saharan: Dar al Gani 1040, NWA 2140, NWA 3118 , and Sahara 98044). They confirmed that the porosities of CV chondrites are indeed very variable, ranging from $0.6 \%$ to $27.7 \%$ (average $=14.6 \%$ ). However, there was no good agreement for the porosities measured for individual meteorites in these two studies, suggesting that the intra-sample heterogeneities are potentially comparable to the inter-sample ones. These discrepancies were particularly large for the reduced CV chondrites Efremovka and Vigarano. In fact, Macke et al. (2011) identified a sharp difference in the porosity of oxidized $\mathrm{CV}_{\mathrm{o}}$ compared with the reduced $\mathrm{CV}_{\mathrm{r}}$ chondrites (average $19.7 \%$ versus $3.6 \%$ porosity).

Corrigan et al. (1997) found that the liquid permeability to be highly variable for seven CV chondrites (Allende, Bali, Efremovka, Kaba, Leoville, Mokoia and Vigarano). Both the intra-and inter-meteorite values ranged several orders of magnitude. For example, the permeability in one lithology of Vigarano was effectively zero (0-0.0001 mdarcy), while in another they ranged from 0.074-0.094 mdarcy. Allende had a much higher permeability at 0.220.52 mdarcy (Corrigan et al., 1997).

Macke et al. (2011) also measured the density the same $12 \mathrm{CV}$ chondrites. They found while the grain densities are similar across the oxidized and reduced $\mathrm{CV}$ chondrites (average = $3.54 \mathrm{~g} / \mathrm{cm}^{3}$, range $3.25-3.68 \mathrm{~g} / \mathrm{cm}^{3}$ ), there bulk densities of the oxidized $\mathrm{CV}_{\mathrm{o}}$ chondrites are significantly lower than for the reduced $\mathrm{CV}_{\mathrm{r}}$ (i.e., average bulk density for $\mathrm{CV}_{\mathrm{o}} 2.87 \mathrm{~g} / \mathrm{cm}^{3}$ versus $3.37 \mathrm{~g} / \mathrm{cm}^{3}$ the $\mathrm{CV}_{\mathrm{r}}$ - Macke et al., 2011). Macke et al. (2011) suggested that differences in bulk densities were caused by differences in porosity between the reduced and oxidized sub- 
groups.

As in the case of the CM chondrites, the inter-sample magnetic susceptibilities of CV chondrites are highly variable, which has also been attributed to differences in the abundance of magnetic minerals among different CV chondrites (Rochette et al. 2008; Macke et al. 2011). Rochette et al. (2008) analyzed 44 CV chondrites including falls, cold and hot desert finds and obtained average of $\log \chi=4.12 \pm 0.49$ (in $10^{-9} \mathrm{~m}^{3} / \mathrm{kg}$; range 3.07-5.11). The average that Macke et al. (2011) obtained for 12 samples is consistent with that value, $\log \chi=3.93$ (in $10^{-9}$ $\mathrm{m}^{3} / \mathrm{kg}$ ). Macke et al. (2011) analyzed 75 stones originating from $12 \mathrm{CV}$ chondrites and obtained values that are consistent with the Rochette et al. (2008) study: average of $\log \chi=4.08$ (in $10^{-9}$ $\mathrm{m}^{3} / \mathrm{kg}$; range from 3.23 to 4.86). Macke et al. (2011) also found that there was a sharp difference in the magnetization between the CV sub-groups, the $\log \chi=3.84$ and 4.70 for the oxidized and reduced sub-groups, respectively (in $10^{-9} \mathrm{~m}^{3} / \mathrm{kg}$ ).

Finally, Ibrahim (2012) measured the mean compressional wave velocity for CV chondrites to be $1934 \pm 778 \mathrm{~m} / \mathrm{s}$ and the average shear wave velocities equal $1934 \pm 778 \mathrm{~m} / \mathrm{s}$. They found the porosity and velocities are anticorrelated across carbonaceous chondrite groups.

\subsubsection{Primary (Nebular) Characterisitcs of the CV Chondrites}

The CV group constitutes $20 \%$ of carbonaceous chondrites. All known samples are petrologic type 3 . There are three CV3 subgroups (two oxidized and one reduced) that differ in their modal magnetite/metallic-Fe-Ni ratios, modal chondrule/matrix ratios, Ni concentrations in metal and sulfide, O-isotopic compositions, and, in the oxidized subgroups, the variety of secondary minerals that formed during parent-body aqueous alteration. CVR (reduced subgroup) chondrites (e.g., Efremovka, Leoville) contain relatively unaltered chondrules and fine-grained matrix material. In contrast, $\mathrm{CV}_{\text {OxA }}$ (oxidized Allende-like) chondrites (e.g., Allende, Axtell) contain ferroan olivine (Fa30-60), nepheline, sodalite, magnetite and Ni-rich sulfide; $\mathrm{CV}_{\text {OxB }}$ (oxidized Bali-like) chondrites (e.g., Bali, Kaba) contain fayalite (Fa95-100), Ca-Fe pyroxenes (Fs 10-50 W045-50), phyllosilicates (saponite and Na phlogopite), andradite garnet, magnetite and Ni-rich sulfide (McSween, 1977; Weisberg et al., 1997; Krot et al., 1997; Kimura and Ikeda, 1997). Some CV3 chondrites (e.g., Vigarano, Mokoia) are breccias containing mixed lithologies (e.g., Krot et al., 1998). Because all three subgroups have roughly similar distributions in their cosmic-ray exposure ages (Scherer and Schultz, 2000), it is likely that all were derived from the same parent asteroid.

\subsection{Primary (Nebular) Characteristics of CV Chondrites}

The $\mathrm{CV}_{\mathrm{R}}$ subgroup best preserves the primary, nebular characteristics of $\mathrm{CV}$ chondrites. These samples consist (in vol. \%) of $\sim 35 \%$ fine-grained silicate-rich matrix material, $\sim 45-55 \%$ chondrules and chondrule fragments, $\sim 3 \%$ CAIs, $\sim 3 \%$ AOIs, and $\sim 5 \%$ metallic Fe-Ni and sulfide grains outside chondrules (McSween, 1977; Rubin, 2011). Also present within the matrix are $\sim 500$ ppm presolar grains (Huss, 1990) and 3\% organic matter (both low molecular-weight compounds and high molecular-weight macromolecular materials) (e.g., Gilmour, 2005).

\subsection{Chondrules in CV Chondrites:}

$\mathrm{CV}$ chondrules average $\sim 900 \mu \mathrm{m}$ in diameter (Friedrich et al., 2015). The percentages of the major chondrule types are: Type-I porphyritic (88\%), Type-II porphyritic $(6 \%)$, barred olivine (BO) (6\%), radial pyroxene (RP) (0.2\%) and cryptocrystalline (C) $(0.1 \%)$ (Grossman et 
al., 1988; Rubin, 2010, 2011). Also present are rare compound chondrule-CAI objects and Alrich chondrules (about 15\% of which contain relict CAI fragments; Russell et al., 2005).

Type I Chondrules: Type-I chondrules in the CVR chondrites consist typically of 20-150$\mu \mathrm{m}$-size magnesian phenocrysts of olivine (and to a lesser extent, low-Ca pyroxene) surrounded by glassy or devitrified feldspathic mesostasis containing $0.5-5$ vol.\% blebs of metallic Fe-Ni (5-40 $\mu \mathrm{m}$ across). Many Type-I chondrules in oxidized CV chondrites contain 50-500- $\mu \mathrm{m}$-size opaque nodules consisting of magnetite intergrown with Ni-rich metal (typically awaruite), pentlandite and minor merrillite (e.g., Rubin, 1991).

Type II Chondrules: Type-II chondrules in CVR chondrites consist of 20-40- $\mu \mathrm{m}$-size ferroan olivine phenocrysts surrounded by fine-grained mesostasis. Little or no metallic Fe-Ni occurs in these chondrules.

About $50 \%$ of $\mathrm{CV}$ chondrules are surrounded by igneous rims with a median thickness of 200-250 $\mu \mathrm{m}$ (Rubin, 1984, 2010). The rims experienced appreciable melting ( 80\%) and consist mainly of euhedral and subhedral olivine \pm low-Ca-pyroxene grains, rounded blebs of metallic Fe-Ni and/or sulfide and minor feldspathic mesostasis (Rubin, 1984; Krot and Wasson, 1995). Mafic silicate grains in these rims tend to be smaller than chondrule phenocrysts, but larger than those in the matrix. The bulk compositions of the igneous rims more closely resemble average matrix material than their particular enclosed chondrules (Rubin and Wasson, 1987), suggesting that the rims formed from well-mixed nebular dust.

\subsection{Fine-Grained Materials in CV Chondrites}

Fine-Grained Matrix: Although the fine-grained matrices in all CV chondrites contain comminuted chondrule fragments (e.g., Hurt et al., 2012), there are significant differences among the CV subtypes in matrix mineralogy. CVR-chondrite matrices contain primary metallic Fe-Ni, troilite and low-Ca pyroxene. Matrices in oxidized CV chondrites contain a diverse array of secondary phases: those in CVOxA chondrites contain major $\mathrm{Ca}-\mathrm{Fe}$ pyroxene, nepheline, sodalite, pentlandite, lath-like ferroan olivine and minor magnetite; those in CVOxB chondrites contain major phyllosilicates, fayalite, Ca-pyroxene, magnetite, andradite and minor ferroan olivine (e.g., Scott and Krot, 2005). Altered matrix regions in the Vigarano CV3 breccia are depleted in $S$ and Na relative to whole rock (Hurt et al., 2012).

Fine-Grained Chondrule Rims: Fine-grained matrix rims around CV chondrules closely resemble matrix material, but may be finer grained and less porous. Similar rims occur around CV CAIs (e.g., MacPherson and Grossman, 1981; Kornacki and Wood, 1984; Scott et al., 1984). By analogy to CM chondrites (Trigo-Rodriguez et al., 2006), the rims may be shock-compacted matrix material rather than nebular fines that accreted around free-floating chondrules. Matrix material also occurs in CV chondrites as discrete millimeter-size lumps (Scott et al., 1984).

Dark Inclusions: Dark inclusions in CV chondrites are millimeter-to-centimeter-size, matrix-rich, lithic clasts that tend to be heavily aqueously altered. There are two types of dark inclusions (Johnson et al., 1990; Krot et al., 1995): Type-A dark inclusions contain smaller and fewer chondrules and CAIs than their CV hosts. One of these is shown in Figure 2.8. Type-B dark inclusions are highly altered; they are free of chondrules and CAIs and instead contain aggregates of relatively fine-grained ferroan olivine (probably chondrule pseudomorphs) surrounded by very small olivine grains. One of these is shown in Figure 2.9, which Zolensky (2002) has proposed originated in an asteroid pond deposit. Some dark inclusions are surrounded by fine-grained matrix-like rims (Kracher et al., 1985). I-Xe dating shows that alteration occurred in some CV dark inclusions before it occurred in their CV hosts (Hohenberg et al., 
2001). At least some of the dark inclusions may be foreign clasts that accreted to the CV parent body. However, the limited oxygen isotope measurements made on dark inclusions to date suggest otherwise (Greenwood et al., 2017).

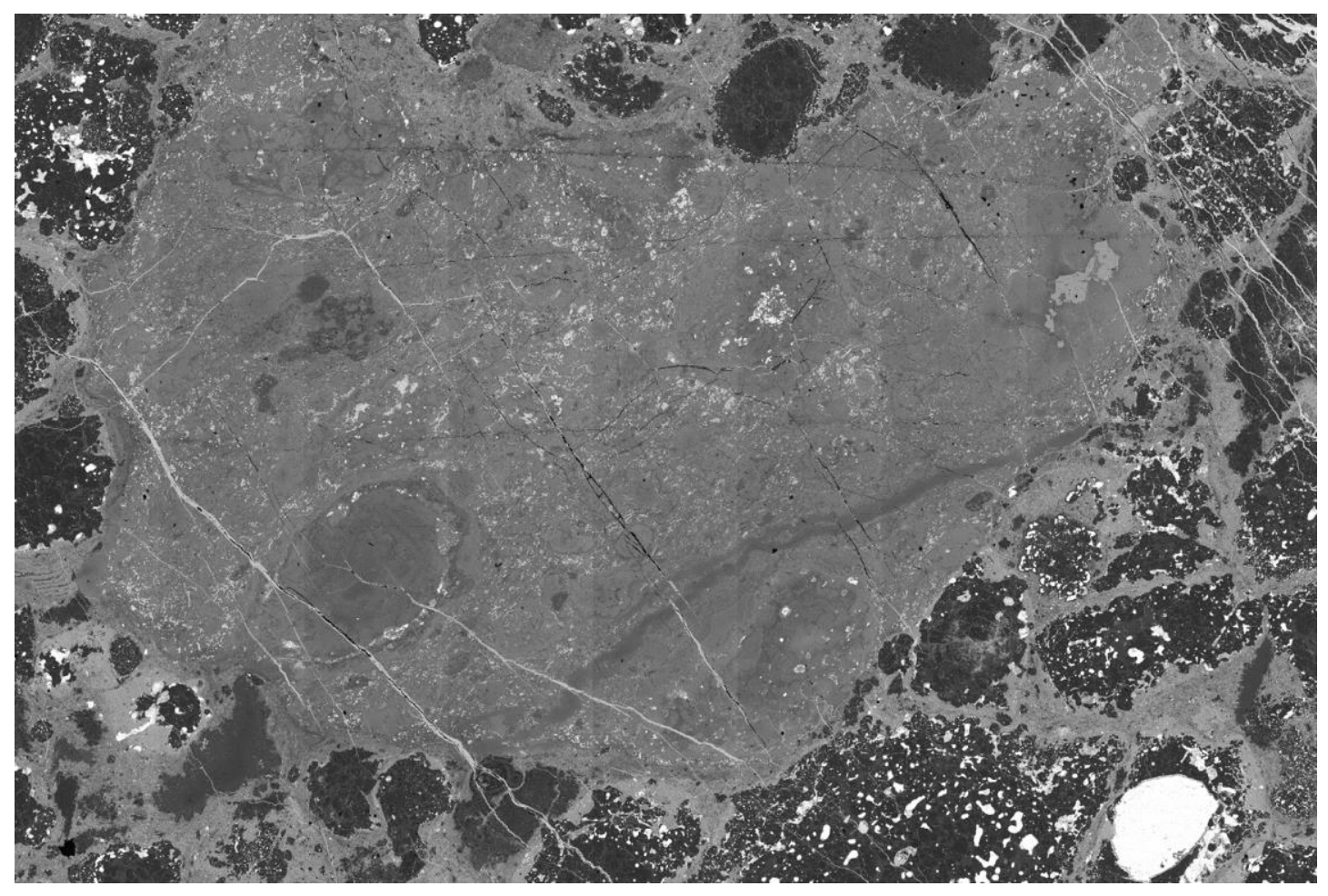

Fig. 2.8. BSE image of a Type A dark inclusion in the Leoville CV chondrite measuring $\sim 6$ $\mathrm{mm}$ across. Veins and altered CAI and chondrules are apparent.

\subsection{Refractory Inclusions in CV Chondrites}

CAIs: CAIs are the oldest objects within chondrites that formed in the solar system; they are 4.658 Ga old (e.g., Amelin et al., 2002; Bouvier and Wadhwa, 2010). Their period of formation may have been as short as 20 - $50 \mathrm{ka}$ (Bizzarro et al., 2004; Jacobsen et al., 2008). Only presolar grains are older.

The major CAI types in unaltered CV chondrites include:

Compact Type-A (CTA) inclusions - compact, spheroidal objects typically ranging from a few hundred micrometers to $\sim 3 \mathrm{~mm}$ in size. Melilite is the most abundant phase, (typically constituting 80-85 vol.\%, but ranging up to nearly 100 vol.\%; e.g., Grossman, 1975, 1980; Simon et al., 1999). Common minor phases include spinel, perovskite, Al-Ti diopside (fassaite) and rhönite.

Fluffy Type-A (FTA) inclusions - contorted, porous objects, most ranging from $0.3-3$ $\mathrm{cm}$ in size. The centimeter-size FTAs are aggregates of numerous melilite-rich nodules, many of which closely resemble individual CTAs; each nodule has its own

Wark-Lovering rim (e.g., MacPherson and Grossman, 1984; MacPherson, 2005).

Type B1 inclusions - coarse-grained spheroidal objects consisting of a melilite mantle surrounding a core of pyroxene, spinel, anorthite, metallic Fe-Ni and noble metal nuggets (e.g., MacPherson et al., 1988). They range in size from several millimeters to several centimeters. 
Type B2 inclusions - spheroidal objects with no melilite mantle that are mineralogically similar to the cores of Type-B1 inclusions (MacPherson et al., 1988).

Type B3 inclusions, also known as forsterite-bearing Type-B inclusions (FoB) - objects containing coarse forsterite grains along with pyroxene, spinel, melilite, and in some cases, minor anorthite (Wark et al., 1987; Petaev and Jacobsen, 2009). These are the least-common variety of Type-B inclusions.

Type $\mathrm{C}$ inclusions - plagioclase- and pyroxene-rich objects with poikilitic or ophitic igneous textures; they are typically several millimeters in size. They contain coarse grains of AlTi diopside (fassaite) and some spinel with a fine-grained anorthitic groundmass; little or no melilite is present (e.g., Scott and Krot, 2005; Krot et al., 2007).

Fine-grained inclusions - porous inclusions containing 1-20- $\mu \mathrm{m}$-size grains of spinel and pyroxene. Most have irregular shapes and range up to $1 \mathrm{~cm}$ in size. Many are composed of spinel nodules surrounded by Wark-Lovering rims and mantled by fine-grained accretionary rims (Brearley and Jones, 1998). There are zoned and unzoned varieties of fine-grained CAIs. Zoned objects have cores composed of spinel, Al-Ti diopside, anorthite, minor forsterite and, in some cases, accessory perovskite and hibonite. These are mantled by nested layers rich in anorthite, melilite, spinel and pyroxene (Brearley and Jones, 1998; MacPherson, 2005). Unzoned objects are smaller and depleted in anorthite. The fine-grained CAIs are enriched in refractory lithophiles, but depleted in refractory siderophiles relative to CI chondrites (Grossman and Ganapathy, 1976).

FUN inclusions - objects containing isotopes exhibiting Fractionated and Unidentified Nuclear effects, i.e., non-radiogenic anomalies (Wasserburg et al., 1977). FUN inclusions vary in mineralogy and texture (MacPherson et al., 1988) and appear to have contained little or no 26Al when they formed (MacPherson et al., 2005). 


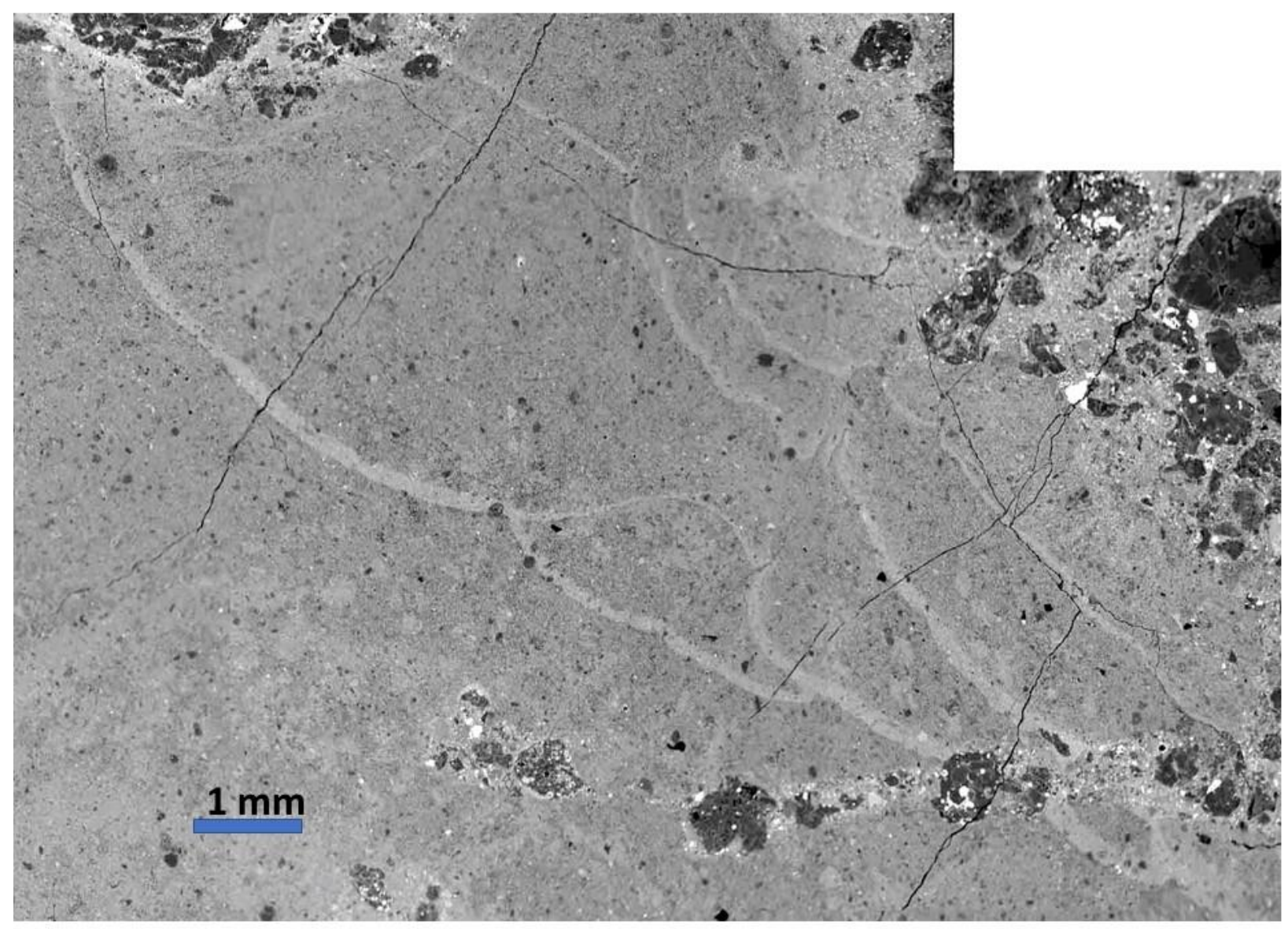

Fig. 2.9. BSE image of a Type B dark inclusion in the Vigarano CV chondrite measuring 2 $\mathrm{cm}$ across. Light-collored sedimentary crossbeds are apparent.

Wark-Lovering rims around CAIs are generally $\sim 10-50-\mu \mathrm{m}$ thick and are relatively uniform around the periphery of the enclosed CAIs. They consist of a series of mono- or bimineralic layers of melilite, spinel and pyroxene associated with some hibonite and perovskite (Wark and Lovering, 1977). The rims likely formed by a multistage process that included flash melting of the surface of the underlying CAI (Wark and Boynton, 2001).

The O-isotopic compositions of whole CAIs and individual CAI minerals plot along a line of slope $\sim 0.95$, i.e., the carbonaceous-chondrite-anhydrous-minerals (CCAM) line, on the standard three-isotope diagram $-\delta 170$ vs. $\delta 180$ (e.g., MacPherson, 2005). Spinel and pyroxene are most enriched in 160; anorthite and melilite tend to be poorer in 160. Secondary phases in oxidized CV chondrites, formed during aqueous alteration (e.g., sodalite, nepheline, andradite, Ca-Fe pyroxene), are typically not enriched in 160 (Clayton et al., 1977). The O-isotopic compositions of FUN inclusions do not lie along the CCAM line. They have higher $\delta 18 \mathrm{O}$ values and may lie along mass-fractionation lines anchored on the CCAM line; these compositions may have resulted from a complicated series of processes involving melt distillation, Rayleigh-type isotopic fractionation and exchange with 16O-poor nebular gas (Clayton et al., 1984).

Most CV CAIs (like most CAIs in other chondrite groups) have $26 \mathrm{Mg}$ excesses relative to terrestrial standards indicating that the CAIs once contained live $26 \mathrm{Al}$ ( $\mathrm{t}^{1} / 2=730,000$ years). The initial 26Al/27Al ratio appears to have been approximately $5 \times 10-5$ (MacPherson et al., 
1995; MacPherson, 2005). FUN inclusions appear to have no 26Mg excesses and there are three principal explanations for this absence: (1) The FUN inclusions formed after 26Al had decayed away. (2) 26Al was heterogeneously distributed in the solar nebula and FUN inclusions formed in a region with a low initial 26Al/27Al ratio. (3) FUN inclusions predate the hypothetical injection of 26Al into the nascent solar system from a nearby supernova or from the explosion of an asymptotic giant branch (AGB) star (e.g., Wood, 2004).

There are five basic REE patterns exhibited by whole CAIs (Mason and Martin, 1977). Three of the patterns are essentially flat (i.e., unfractionated) and differ in their enrichments or depletions in the volatile REEs - Eu and Yb. The so-called Group II pattern shows depletions in refractory (e.g., Ho, Er) and volatile $(\mathrm{Eu}, \mathrm{Yb}) \mathrm{REEs}$ and can be produced only by fractional condensation (Boynton, 1975; Davis and Grossman, 1979; MacPherson and Davis, 1994). This pattern is present in some Type-C CAIs and is the most common one among fine-grained spinelrich CAIs.

Models for CAI formation, generally involving condensation, melting, evaporation and isotopic exchange, were reviewed by MacPherson et al. $(1988,1995,2005)$ and MacPherson (2005). The similarities in O-isotopic compositions among CAIs in different chondrite groups and their wide distribution in the solar system are explained by models in which CAIs were formed near the Sun and then transported to different heliocentric distances by such processes as jets, turbulent diffusion, gravitational instabilities and advection due to viscous expansion of the nebular disk (e.g., Gounelle et al., 2001; Shu et al., 2001; Cuzzi and Hogan, 2003; Cuzzi et al., 2003, 2005; Weidenschilling, 2003; Boss, 2004; Wood, 2004; Cuzzi and Weidenschilling, 2006; Ciesla, 2009, 2010; Jacquet et al., 2011).

The majority of coarse-grained (non-FUN) CAIs may have related origins. One possibility suggested by Rubin (2012) is that a large fraction of the original CAIs that formed near the Sun were sub-millimeter-size CTA inclusions that experienced surficial melting and acquired Wark-Lovering rims. These objects were then transported throughout the nebula. Moderate numbers of individual rimmed CTA inclusions agglomerated together along with small amounts of 16O-rich mafic dust to form FTA inclusions after undergoing minor melting. Some CTA inclusions clumped together along with varying amounts of 16O-rich forsteritic dust (and perhaps some anorthite); after experiencing extensive melting and partial evaporation, these processed objects became Type-B inclusions. If $10-15 \%$ forsterite was present prior to melting, B2 inclusions formed; if $\sim 25 \%$ forsterite was present, B3 inclusions formed. Some B2 inclusions collided with and stuck to melilite-rich CTAs; those assemblages that later experienced high-temperature processing became Type-B1 CAIs. The presence of relict Type-B CAIs within some Type-C CAIs led Krot et al. (2007) to suggest that Type-C inclusions formed by the melting of coarse-grained Type-B CAIs.

AOA in CV Chondrites: Amoeboid olivine aggregates (AOAs), also known as amoeboid olivine inclusions (AOIs), are irregular or lobate-shaped, porous objects, ranging in size in CV chondrites from 0.1 to $10 \mathrm{~mm}$ and averaging $~ 3 \mathrm{~mm}$ (Grossman and Steele, 1976; Simon and Haggerty, 1979; Wark, 1979; Cohen et al., 1983; Kornacki et al., 1983; Kornacki and Wood, 1984; Rubin, 2013). In unaltered CV chondrites, the constituents are major olivine, major or minor $\mathrm{Ca}$ pyroxene, minor anorthite, accessory metallic Fe-Ni and perovskite, and in some cases, accessory spinel \pm low-Ca pyroxene \pm melilite. Some AOAs enclose small CAIs sporting WarkLovering rims (Komatsu, 2003; Krot et al., 2004). Mineral grains in the interiors of AOAs are typically $1-20 \mu \mathrm{m}$ in size; there is an overlying $10-80-\mu \mathrm{m}$-thick rim of coarser $(20-130-\mu \mathrm{m}-$ 
size) olivine grains that, in some cases, form $120^{\circ}$ triple junctions. Rubin (2013) suggested that these rims formed by flash-melting porous olivine-rich rims that surrounded AOA cores.

2.5.3.5.2. Asteroidal Chemical and Mineralogical Processes Affecting the CV Chondrites

All components in CV chondrites were affected by parent-body alteration: matrix, chondrule rims, dark inclusions, chondrule mesostasis, metal and sulfide grains (both inside and outside chondrules) and CAIs.

\subsubsection{Models of Aqueous Alteration Processes Affecting the CV Chondrites}

The presence of phyllosilicates in CVOxB chondrites indicates that parent-body alteration involved an aqueous fluid. There are two plausible sources of water: ice that agglomerated along with silicates beyond the snow line to form CV asteroids (e.g., Warren, 2011a,b; Kruijer et al., 2017a,b,c) and nebular phyllosilicates formed during chondrule formation as volatilized lithophile elements reacted with $\mathrm{H} 2 \mathrm{O}$ in the ambient gas (e.g., Petaev and Wood, 1998; Wasson and Trigo-Rodríguez, 2004; Ciesla and Lauretta, 2005; Wasson, 2008).

The hydrothermal activity responsible for the aqueous alteration of $\mathrm{CV}$ chondrites could have been facilitated by internal heating due to the decay of $26 \mathrm{Al}$ or by collisional heating. Internal heating could have caused fluid flow toward the asteroid surface along a temperature gradient (Young et al., 1999; Young, 2001). It could also have caused convective flow of water through fractures and pores in a permeable body (McSween et al., 2002; Young et al., 2003; Travis and Schubert, 2005). Alternatively, water mobilization could have been due to impactdriven processes involving melting or vaporization of ice or the dehydration of phyllosilicates (e.g., Lange et al., 1985; Rubin, 2012).

Rubin (2012) reported that many CVR and CVOxB chondrites, but few, if any, CVOxA chondrites have petrofabrics. He found that nearly all CV chondrites with petrofabrics are shock-stage S3-S4 and nearly all those that lack petrofabrics are S1. The CVOxB and CVR samples were shocked, became extensively fractured and developed petrofabrics; CV3OxA samples were not shocked and did not develop petrofabrics. The oxidized CV subgroups have much higher porosities (typically 20-28\%) than the CVR subgroup (0.6-8\%). The oxidized CV subgroups formed from higher-porosity materials that facilitated aqueous alteration after water mobilization (Rubin, 2012; MacPherson and Krot, 2014). The CVR chondrites may have formed from low-porosity material that inhibited alteration after water was mobilized (Rubin, 2012). Alternatively, the CVR precursor material experienced intense shock that decreased the wholerock porosity and permeability and volatilized any intergranular water and ice (MacPherson and Krot, 2014).

\subsubsection{Thermal and Hydrothermal Metamorphism in CV Chondrites}

$\mathrm{CV}$ chondrites were subjected to aqueous alteration, Fe-alkali-halogen metasomatism and thermal metamorphism; these were probably not distinct processes. Housley and Cirlin (1983) and Krot et al. (1995) demonstrated that the oxidized CV chondrites experienced alteration on their parent asteroid and that the reduced $\mathrm{CV}$ chondrites best represent the original, minimally altered assemblage. During alteration, a myriad of mineralogical changes occurred: (1) low-Ni metallic Fe underwent oxidation and sulfidation to form magnetite, high-Ni metallic Fe and sulfides, (2) primary phases experienced Fe-alkali-halogen metasomatism to produce nepheline, sodalite, wollastonite, hedenbergite, andradite and other phases, and (3) anhydrous silicates were transformed into phyllosilicates. In the CVOxA chondrites ferroan olivine, nepheline, sodalite, magnetite and Ni-rich sulfide were formed; it is likely that phyllosilicates also formed, but were 
later largely destroyed by thermal metamorphism. In the CVOxB chondrites fayalite, $\mathrm{Ca}-\mathrm{Fe}$ pyroxenes, phyllosilicates, andradite, magnetite and Ni-rich sulfide were formed.

Thermal metamorphism causes irreversible changes in the structure, texture and composition of organic matter. Bonal et al. (2006) used Raman spectroscopy to analyze organic matter in a suite of $\mathrm{CV}$ chondrites and construct a thermal metamorphic scale. They assigned metamorphic subtypes ranging from CV3.1 (Kaba) to CV>3.6 (Allende). Bonal et al. found that progressive changes in organic matter due to metamorphism are largely independent of aqueous alteration. Mokoia (a breccia containing both CVOxA and CVOxB lithologies) has compositionally heterogeneous olivine produced during aqueous alteration (Cohen et al., 1983) as well as a high metamorphic subtype (3.6). This indicates that Mokoia underwent aqueous alteration after it had experienced peak metamorphism (Bonal et al., 2006). (If alteration had preceded metamorphism, the olivine compositional heterogeneity would have been significantly reduced.) In contrast to Mokoia, Allende (CVOxA) was metamorphosed after it was aqueously altered, thereby destroying most of its phyllosilicates (Krot et al., 1998).

Huss et al. (2006) used the Bonal et al. sequence, the characteristics of presolar grains and temperatures inferred from gas-release profiles from presolar diamonds (Huss and Lewis, 1994a,b) to estimate the metamorphic temperatures of several CV3 chondrites: Leoville, $\sim 250^{\circ} \mathrm{C}$; Vigarano, Mokoia, Kaba, Efremovka, 300-400 ${ }^{\circ}$; Grosnaja, $~ 500^{\circ} \mathrm{C}$; Axtell, Allende, 550$600^{\circ} \mathrm{C}$.

\subsubsection{Asteroidal Minerals:}

\subsection{Non-silicates}

The non-silicate minerals in CV chondrites include native metals (e.g., Os, $\mathrm{Cu}$ ), metal alloys (e.g., awaruite, taenite), sulfides (e.g., troilite, pentlandite), oxides (e.g., corundum, hibonite), hydroxides (e.g., pyrochlore), carbonates (e.g., calcite), sulfates (e.g., barite), molybdates (e.g., powellite), tungstates (e.g., scheelite) and phosphates (e.g. apatite, merrillite) (Rubin, 1997; Brearley and Jones, 1998; Rubin and Ma, 2017). These phases occur in chondrules, CAIs, AOAs, dark inclusions and the matrix.

\subsection{Carbonates in CV Chondrites}

Carbonates are not common in $\mathrm{CV}$ chondrites. Nevertheless, calcite occurs as a rare accessory phase in some Allende dark inclusions, deposited along fractures in across sections, and pseudomorphically replacing calcic pyroxenes in Vigarano. Authors have argued that some produced during parent-body aqueous alteration and some during terrestrial weathering (Kurat et al., 1989; Abreu and Brearley 2005). The matrix regions of Bali contain carbonate associated with phosphate and magnetite (Brearley, 2006).

\subsection{Sulfides in CV Chondrites}

Troilite occurs as a primary phase (in many cases associated with metallic $\mathrm{Fe}-\mathrm{Ni}$ ) in the matrix, chondrules and CAIs of some CV chondrites, particularly CVR chondrites (e.g., McSween, 1977; Brearley and Jones, 1998). Pyrrhotite and pentlandite occur as secondary phases associated with magnetite in opaque nodules in Allende (e.g., Rubin, 1991) and other $\mathrm{CV}_{\text {OxA }}$ chondrites. However, most of the sulfide in Axtell ( $\left.\mathrm{CV}_{\text {OxA }}\right)$ is troilite (Casanova and Simon, 1994). Millerite occurs in some Type-B CAIs (Brearley and Jones, 1998). Fremdlinge 
in some CAIs contain several sulfides - pentlandite, molybdenite, heazlewoodite, pyrrhotite and FeS-NiS monosulfide solid solution (Brearley and Jones, 1998).

\subsection{Fe-Oxides and Phosphates in CV Chondrites}

Fe-oxides and phosphates in $\mathrm{CV}$ chondrites are secondary phases, formed during aqueous alteration. Magnetite and magnesiowüstite have been reported in some Type-B CAIs; some Fremdlinge contain these phases as well as V-rich magnetite (Brearley and Jones, 1998). Cohen et al. (1983) reported magnetite in AOAs in Mokoia. Ilmenite is a rare phase, present in CAIs in some oxidized CV chondrites; in some cases, it appears to be replacing perovskite. Magnetite and ilmenite occur in the matrices of oxidized CV chondrites. Allende chondrules contain large opaque nodules containing major magnetite surrounding grains of awaruite and pentlandite (e.g., Rubin, 1991).

Merrillite occurs as a nearly continuous ring near the margins of some of the opaque nodules in Allende (Rubin, 1991). Merrillite is also present, along with apatite, in some Fremdlinge in Allende CAIs (Brearley and Jones, 1998). Ca-phosphate (probably merrillite) also occurs in dark inclusions in Allende.

\subsection{Asteroidal Minerals in CV Chondrites: Silicates}

Silicates from all six structural groups occur in CV chondrites: nesosilicates (e.g., andradite, forsterite), sorosilicates (e.g., melilite), cyclosilicates (e.g., cordierite), inosilicates (e.g., Al-Ti diopside, grossmanite), phyllosilicates (e.g., saponite, clintonite) and tectosilicates (e.g., anorthite, nepheline) (e.g., Keller and Buseck 1990b; Rubin and Ma, 2017).

Primary silicates occur in chondrules, AOAs, CAIs and the matrix of CV chondrites. These phases include olivine, enstatite, orthopyroxene, diopside, Al-Ti diopside, rhönite, melilite, cordierite and anorthite. Secondary silicates are present in all major components of CV chondrites, particularly the oxidized subgroups. These phases include ferroan olivine and fayalite, hedenbergite, diopside and salite, nepheline, sodalite, grossular, andradite, wollastonite, monticellite, secondary anorthite, secondary melilite and phyllosilicates.

\section{Phyllosilicates in CV Chondrite Matrices}

In $\mathrm{CV}_{\mathrm{OxB}}$ chondrites, matrix olivine grains have been replaced by saponite. Allende has a very low water content (<0.2 wt.\%; Jarosewich et al., 1987) and apparently contains no phyllosilicates or other hydrous minerals in the matrix. CV-chondrite dark inclusions also generally lack phyllosilicates.

\section{Phyllosilicates Replacing CAIs and Chondrule Mesostases}

Altered chondrules and CAIs in $\mathrm{CV}_{\mathrm{OxB}}$ chondrites contain several phyllosilicates including Fe-bearing saponite, Na phlogopite, Al-rich serpentine and Na-K mica (Brearley, 2006). There are rare phyllosilicates in Allende CAIs - phlogopite, montmorillonite, clintonite, margarite, saponite and chlorite (Brearley, 2006). Phyllosilicates are present in some chondrules in Mokoia (saponite, Na phlogopite, serpentine), Kaba (saponite) and Grosnaja (chlorite, saponite) (Krot et al., 1995).

The phlogopite, clintonite, margarite and chlorite in Allende CAIs have higher thermal stabilities than the phyllosilicates in $\mathrm{CV}_{\text {OxB }}$ chondrites (Brearley, 2006), consistent with the higher inferred thermal metamorphic temperature of Allende. 


\subsection{Terrestrial Chemical and Mineralogical Processes Affecting the CV Chondrites}

Terrestrial alteration of meteorites begins during atmospheric passage when fusion crust is produced. Magnetite in the fusion crust forms from the oxidation of metallic Fe-Ni and sulfide in the falling meteoroid. Terrestrial water (moist air and precipitation) can cause oxidation of metallic Fe-Ni, sulfide and silicates in CV chondrites after these rocks reach the Earth's surface. [Oxide phases are generally more resistant to terrestrial weathering.] Weathering of CV whole rocks occurs in all terrestrial environments including hot deserts, cold deserts and even museum cabinets (e.g., Bagnall, 1991).

Evaporite deposits occur on the surfaces of $24 \%$ of Antarctic CV chondrites (Losiak and Velbel, 2011). Insolation heating of these meteorites on summer days with little wind is aided by their dark fusion crust; this can lead to the melting of ice, leaching of minerals and migration of mineral-laden water to the meteorite surface via pores and fractures.

\subsection{Terrestrial Non-Silicates in CV Chondrites}

There is little published information on terrestrial weathering products of CV chondrites. Axtell ( $\left.\mathrm{CV}_{\text {OxA }}\right)$ contains a $\mathrm{S}$ - and Ni-bearing phase that appears to be a terrestrial weathering product of pentlandite (Simon et al., 1995). Metallic Fe-Ni in some $\mathrm{CV}_{\mathrm{R}}$ finds has been altered to goethite. Abreu and Brearley (2005) idenfied Ca-carbonate veins in a section from a stone of Vigarano that had been briefly exposed to weathering and argued that these carbonates were terrestrial in origin. In addition, evaporitic phases on the surfaces of CV chondrites include $\mathrm{Mg}$ and Ca-carbonates (including amorphous Mg-carbonate) and sulfates (Losiak and Velbel, 2011).

\subsection{Terrestrial Silicates in CV Chondrites}

Highly weathered CV3 chondrites contain phyllosilicates, formed from mafic silicates via oxidation and hydration.

\subsubsection{Chemical Composition and Mineralogy of CK Chondrites and Processes Affecting these Meteorites}

Unlike other carbonaceous chondrite (CCs) groups that contain only unequilibrated material (petrologic types 1 to 3), CK (Karoonda-like) chondrites are mostly dominated by equilibrated meteorites (petrologic types 4 to 6). Only two falls were observed so far, the CK4 Karoonda and Kobe. However, over the last fifteen years, systematic collection efforts for meteorites in the Saharan and Antarctic deserts have resulted in the recovery of a significant number of new CKs, especially of petrologic type 3 or at the type $3-4$ transition. Through the study of new samples, many similarities were found between the $\mathrm{CK}$ and $\mathrm{CV}$ chondrites, hence the recognition of the CV-CK clan (Weisberg et al., 2006).

\subsection{Physical Properties of CK chondrites}

Macke et al. (2011) studied the physical properties of five CK chondrites (Karoonda (replicates), Maralinga (replicates), NWA 2388, NWA 2867, and NWA 5515). They determined the average bulk density of CK chondrites to be $3.00 \pm 0.11 \mathrm{~g} / \mathrm{cm}^{3}$ and the grain density $3.55 \pm$ $0.04 \mathrm{~g} / \mathrm{cm}^{3}$.

The average porosity by meteorite is $14.0 \%$. However, Macke et al. (2011) noted that 
there was significant variability in porosity. They suggested that these variations resulted from differences in their terrestrial weathering features, with the more weathered samples having lower porosities.

Corrigan et al. (1997) reported that the liquid permeability of CK4 Maralinga was 4.546.72 mdarcy, which is several orders of magnitude higher than most other chondrites.

Rochette et al. (2008) examined 27 CK chondrites including falls, hot and cold desert finds, and samples recording the full range of thermal metamorphism. They measured the average magnetization of these samples to be $\log \chi=4.62 \pm 0.14$. Macke et al. (2011) obtained values that are within error of the results from Rochette et al. (2008), $\log \chi=4.67 \pm 0.02(\log \chi$ $*$ in $\log 10^{-9} \mathrm{~m}^{3} / \mathrm{kg}$ ). Macke et al. (2011) noted that the grain density and magnetization for CK chondrited plotted in the same region as the L/LL ordinary chondrites.

Ibrahim (2012) measured the mean compressional wave velocity for CK chondrites to be $1049 \pm 327 \mathrm{~m} / \mathrm{s}$ and the average shear wave velocities equal $1049 \pm 327 \mathrm{~m} / \mathrm{s}$. They found that in general the porosity and velocities are anticorrelated across carbonaceous chondrite groups.

\subsection{Primary (Nebular) Characteristics of the CK Chondrites}

\subsection{Bulk compositions}

As initially reported by Kallemeyn et al. (1991), elemental abundance patterns in CK chondrites are similar to those in CV chondrites, and to a lesser extent, in CO chondrites. In unequilibrated CK3 chondrites, abundances of refractory lithophile elements display broadly similar variations than those measured in CV chondrites (Greenwood et al., 2010a). Equilibrated (types 4 to 6) $\mathrm{CK}$ chondrites have refractory lithophile (e.g., Al, Sc, Ca) and refractory siderophile (Os, Ir, and Ru) abundances of $\sim 1.2 \times$ CI (Kallemeyn, 1991; Isa et al., 2011), between those measured in $\mathrm{CO}$ and $\mathrm{CV}$ chondrites. Moderate volatile element abundances are lower to those in CV chondrites (with the exception of Mn and Na; Wasson et al., 2013) while the most volatile elements (e.g., As, Sb) display abundances of around 10 to $20 \%$ lower than CV values (Kallemeyn, 1991; Wasson et al., 2013). Br is $4 \times$ lower in equilibrated CK chondrites than in CV chondrites (Wasson et al., 2013), as well as $\mathrm{Bi}(\sim 3 \times)$ and $\mathrm{Tl}(\sim 30 \times)$ (Isa et al., 2011).

In term of oxygen isotope ratios, CK3-6 chondrites display values ranging between $2.24 \pm 0.16 \%$ o $(1 \sigma)$ and $1.56 \pm 0.01 \%$ in $\delta^{18} \mathrm{O}$ and between $-5.91 \pm 0.10 \%$ and $-2.53 \pm 0.41 \%$ in $\delta^{17} \mathrm{O}$ that plot along the CCAM line (e.g., Geiger et al., 1993; Clayton and Mayeda, 1999; Ivanova et al., 2000, 2003; Zipfel et al., 2000; Brandstätter et al., 2003; Greshake et al., 2003; Greenwood et al., 2010a). These values are similar to those measured for the $\mathrm{CV}_{\mathrm{R}}$ subgroup, while the $\mathrm{CV}_{\text {OxA }}$ subgroup is slightly shifted towards heavier values (Greenwood et al., 2010a). In contrast, the $\mathrm{CV}_{\text {Охв }}$ display a significant displacement to isotopically heavier values as the result of more extensive aqueous alteration than the $\mathrm{CV}_{\mathrm{R}}, \mathrm{CV}_{\mathrm{OxA}}$, and $\mathrm{CK}$ chondrites experienced.

Contrary to oxygen isotopic compositions, measurements of mass-independent nucleosynthetic anomalies in ${ }^{54} \mathrm{Cr}$ and ${ }^{50} \mathrm{Ti}$ are characterized by large uncertainties that make difficult the CV-CK differentiation (Shukolyukov and Lugmair, 2006; Trinquier et al., 2007; Qin et al., 2010). Yin et al. (2017) reported an $\varepsilon^{54} \mathrm{Cr}$ average value of $+0.66 \pm 0.06(2 \sigma)$ for equilibrated $\mathrm{CK}$ chondrites, a value lower than the average $\varepsilon^{54} \mathrm{Cr}$ value of $\mathrm{CV}$ chondrites 
$(+0.88 \pm 0.06)$. Because of its higher value $(+1.83 \pm 0.09)$, Yin et al. (2017) excluded from the CK average the CK3 NWA 6047 that deviates from equilibrated CK.

\subsection{Chondrules in CK Chondrites:}

Although an initial abundance of chondrules estimated between 10 and 15 vol\% by Kallemeyn et al. (1991), recent studies indicate that CK chondrites can contain up to $\sim 50 \%$ of chondrules in types 3-4 (Tomeoka et al., 2005; Chaumard and Devouard, 2016). Apparent diameters of chondrules in CK chondrites range approximately from 0.16 to $7.5 \mathrm{~mm}$ (Geiger et al., 1993; Zipfel et al. 2000; Bukovanská et al., 2003; Smith and Russell, 2003; Rubin 2010), with an average diameter of $\sim 0.8 \mathrm{~mm}$ (Greenwood et al., 2010a; Chaumard and Devouard, 2016) (up to $1.05 \mathrm{~mm}$ in the CK4 Tnz 057; Chaumard and Devouard, 2016). Igneous rims were described around many chondrules in the two CK3 NWA 1559 and DaG 431 (Zipfel et al. 2000; Rubin 2010; Wasson et al., 2013), as well as around up to 60\% of chondrules in the CK3-4 NWA 4727, NWA 4425, NWA 4423, NWA 4422, and Tnz 057 (Chaumard and Devouard, 2016). As described in other CCs (Rubin, 1984a,b; Rubin and Wasson, 1987; Krot and Wasson, 1995), most of the igneous rims in CK chondrites have microporphyritic textures, with thicknesses ranging from $\sim 20 \mu \mathrm{m}$ to $\sim 2 \mathrm{~mm}$ (Rubin, 2010; Chaumard and Devouard, 2016). They have a mineralogy dominated by $\mathrm{Ca}$-rich pyroxene, olivine, and plagioclase in various proportions (Chaumard and Devouard, 2016), often associated with sulfides and magnetite that also rim chondrules (Rubin, 2010; Wasson et al., 2013). In equilibrated CK, the lack of igneous rims around chondrules was interpreted as the consequence of the parent body metamorphic event (Smith and Russell, 2003).

Porphyritic olivine and olivine-pyroxene (PO, POP), granular olivine (GO), barred olivine and olivine-pyroxene (BO, BOP), and radial pyroxene (RP) chondrules were described in the CK3 chondrites Watson 002, NWA 1559, DaG 431, NWA 4724, NWA 4425, and in several CK4-6 (Kallemeyn et al., 1991; Keller et al., 1992; Geiger et al., 1993; Brandstätter et al., 2003; Bukovanská et al., 2003; Tomeoka et al., 2005; Pratesi et al., 2006; Rubin 2010; Chaumard and Devouard, 2016).

Since most of the CK chondrites are equilibrated, any chondrule with no secondary alteration features has been reported so far. In CK3, chondrule olivines display Fe-poor cores and Fe-rich rims (often containing pits, voids, and tiny inclusions of opaque phases) (e.g., Davidson et al., 2014c; Chaumard and Devouard, 2016). These chemical Fe/Mg zonations were formed by the chemical re-equilibration of chondrules with the surrounding Fe-rich matrix and their recrystallization during metamorphism (e.g., Greenwood et al., 2010a; Chaumard and Devouard, 2016). In consequence, there is no accurate estimate of the type I/type II ratio in CK chondrites. However, based on their size, texture, and mineralogy, Chaumard and Devouard (2016) estimated that between 40 and $85 \%$ of chondrules in several CK3 chondrites and Tnz 057 (CK4) might correspond to former type I chondrules.

Most chondrules in CK chondrites are dominated by olivine. In CK3 and some CK4 chondrites, olivines in FeO-poor chondrules are chemically zoned, with core compositions ranging between nearly pure forsterite and $\sim \mathrm{Fa}_{0.5}$ and rims equilibrated at $\sim \mathrm{Fa}_{31}$ (e.g., Rubin et al., 1988; Noguchi, 1993; Davidson et al., 2014c; Chaumard and Devouard, 2016; Dunn et al., 2016b). $\mathrm{CaO}, \mathrm{Cr}_{2} \mathrm{O}_{3}$ and $\mathrm{Al}_{2} \mathrm{O}_{3}$ contents in olivine decrease during metamorphism, while $\mathrm{NiO}$ and $\mathrm{MnO}$ contents increase (Dunn, 2014; Chaumard and Devouard, 2016), to reach the values 
measured in equilibrated $\mathrm{CK}$ chondrites, i.e., $\sim 0.01 \mathrm{wt} \% \mathrm{Cr}_{2} \mathrm{O}_{3}$ and $\sim 0.51 \mathrm{wt} \% \mathrm{NiO}$ (e.g., Noguchi, 1993; Davidson et al., 2014c).

CK chondrules contains Low-Ca pyroxene as phenocrysts, with compositions ranging from nearly pure enstatite to $\sim \mathrm{Fs}_{25}$, as well as high-Ca pyroxene as a minor to accessory phase ( Fs10) (e.g., Keller et al., 1992; Noguchi, 1993; Tomeoka et al., 2005).

Mesostases in CK chondrules are microcrystalline and mainly composed of a finegrained mixture of plagioclase and pyroxene, occasionally with small amount of magnetite and augite (Kallemeyn et al., 1991; Noguchi, 1993; Chaumard and Devouard, 2016).

Several rounded objects with diameters up to $\sim 1 \mathrm{~mm}$ were described as magnetite-sulfide chondrules in some CK chondrites (Rubin, 1993; Geiger and Bischoff, 1995). Displaying granular textures and concentric layering, they are mainly composed of magnetite that contains exsolution lamellae of ilmenite and spinel (Geiger and Bischoff, 1995). These objects also contain pentlandite, with minor amount of chloroapatite, as observed in magnetite-sulfide nodules within mafic silicate chondrules (Rubin, 1993).

\subsection{Fine-Grained Materials in CK Chondrites}

By comparison to matrices in other groups of CCs, matrices in $\mathrm{CK}$ chondrites are coarsegrained due to parent body metamorphism. Matrix silicates have average sizes $<10 \mu \mathrm{m}$ in unequilibrated CK chondrites (e.g., in the CK3 NWA 1559; Wasson et al., 2013), 10-50 $\mu \mathrm{m}$ in CK4, 50-200 $\mu \mathrm{m}$ in CK5, and up several hundreds of $\mu \mathrm{m}$ in CK6 (e.g., Kallemeyn et al., 1991; Noguchi et al., 1993; Chaumard et al., 2009a). Many grains are platy, elongated and/or tabular in CK3, become more globular in CK3.9/4, then define a granular recrystallized texture in higher petrologic types (e.g., Kallemeyn et al., 1991; Brearley et al., 2009; Chaumard et al., 2009a).

The mineralogy of CK chondrite matrices is dominated by olivine and plagioclase, with small amounts of low-Ca and high-Ca pyroxene, magnetite, and sulfides (e.g., Scott and Taylor, 1985; Kallemeyn et al., 1991; Keller et al., 1992; Rubin, 1992; Noguchi et al., 1993; Tomeoka et al., 2005).

In the unequilibrated $\mathrm{CK} 3$ chondites, matrix olivines have variable compositions, $\sim \mathrm{Fa}_{33}-$ $\mathrm{Fa}_{39}$, NiO contents ranging from $\sim 0.3$ to 0.7 wt.\% (Brandstätter et al., 2003; Chaumard et al., 2009a; Dunn, 2011; Dunn et al., 2016), and contain small amounts of MnO (e.g., 0.3 wt\% in NWA 1559; Brandstätter et al., 2003). High-Ca pyroxene in CK3 chondrites has diopsidic compositions ( $\mathrm{Fs}_{5.7}$ to $\left.\mathrm{Fs}_{11}\right)$.

In CK4-5 chondrites, matrix olivines are chemically homogeneous, equilibrated at $\sim \mathrm{Fa}_{31}$, and Ni-rich (from 0.3 to 0.7 wt.\% NiO) (e.g., Scott and Taylor, 1989; Geiger and Bischoff, 1991; Kallemeyn et al., 1991; Keller et al., 1992; Noguchi, 1993; Chaumard et al., 2009a; Greenwood et al., 2010a; Dunn, 2011; Dunn et al., 2016b). Olivine grains also contain significant amounts of $\mathrm{MnO}$ (0.18-0.26 wt\%; Noguchi, 1993). Both low-Ca and high-Ca pyroxenes (augitic compositions) were reported in equilibrated CK chondrites, with compositions of Fs 17-27 and Fs8-12, respectively (Kallemeyn et al., 1991; Geiger and Bischoff, 1991; Noguchi, 1993). Low-Ca pyroxene has low $\mathrm{TiO}_{2}(<0.1 \mathrm{wt} \%)$ and $\mathrm{Cr}_{2} \mathrm{O}_{3}(<0.15 \mathrm{wt} \%)$ contents, and high $\mathrm{Al}_{2} \mathrm{O}_{3}$ (up to $\left.\sim 3.5 \mathrm{wt} \%\right), \mathrm{MnO}(\sim 0.4 \mathrm{wt} \%)$, and $\mathrm{CaO}(\sim 0.6-1.0 \mathrm{wt} \%)$ contents (Noguchi, 1993). Augitic pyroxenes contain $\sim 0.2 \mathrm{wt} \% \mathrm{MnO}$ and $\sim 1.8-2.6 \mathrm{wt} \% \mathrm{Al}_{2} \mathrm{O}_{3}$ (Noguchi, 1993). 
Plagioclase is virtually absent or microcrystalline in CK3, while their mean sizes are generally $<4 \mu \mathrm{m}$ in CK4, $4-50 \mu \mathrm{m}$ in CK5, and > $50 \mu \mathrm{m}$ in CK6 (Kallemeyn et al., 1991; Huss et al., 2008; Chaumard et al., 2015). Plagioclase grains are often zoned in CK matrices, hence the measurement of large compositional variations $\left(\sim \mathrm{An}_{10}\right.$ to $\left.\mathrm{An}_{100}\right)$ (e.g., Scott and Taylor, 1985; Geiger and Bischoff, 1991; Kallemeyn et al., 1991; Rubin, 1992; Keller, 1993; Noguchi et al., 1993; Chaumard et al., 2015). As proposed for OC breccias, Kallemeyn et al. (1991), Rubin (1992), and Tomeoka et al. (2005) suggested that heterogeneities in plagioclase compositions reflect a chemical fractionation caused by the crystallization of a shock-induced melt that imply the formation of plagioclasic feldspar (calcic then sodic) then sodic plagioclase and K-feldspar (e.g., Bischoff et al. 1983). Chaumard and Devouard (2015) suggested that zoned plagioclases (calcic cores and sodic borders) in CK matrices were formed during parent body metamorphism, by reaction between pyroxene and spinel to form calcic plagioclase, then by the addition of $\mathrm{Na}$ possibly due to the destabilization of hydrated phases to form sodic plagioclase. In contrast to Chaumard and Devouard (2015), Keller (1993) and Noguchi (1993) reported reverse zoning (sodic cores and calcic borders). Keller (1993) suggested that chemical compositions measured in plagioclases from Maralinga (CK4) are consistent with the subsolidus relations proposed by Grove et al. (1983) in the plagioclase solid solution series. At equilibrium, a miscibility gap occurs from approximately $\mathrm{An}_{40}$ to $\mathrm{An}_{90}$ between 400 and $575^{\circ} \mathrm{C}$. Then, calcic rims of plagioclase from Maralinga would have been produced by prograde reactions during parent body thermal metamorphism (Keller, 1993). According to the crystallization sequence described for OC breccias and the reverse zoning observed by Keller (1993), Noguchi (1993) proposed that calcic borders of plagioclases were formed during a thermal metamorphic event while sodic cores were inherited from the nebula.

On of the most striking feature of CK chondrites (mostly in the equilibrated CK4-5 chondrites) is the texture of their matrices. Matrix olivine and plagioclase grains contain numerous micrometer- and nanometer-sized vesicles and inclusions of opaque phases such as magnetite and Fe,Ni sulfides (Kallemeyn et al., 1991; Rubin, 1992; Geiger and Bischoff, 1995; Tomeoka et al., 2001, 2005; Neff and Righter, 2006; Ohnishi et al., 2007; Brearley, 2009; Chaumard and Devouard, 2015). These textures have been compared to silicate darkening (Rubin, 1991, 1992; Tomeoka et al., 2001, 2005), a feature commonly observed in OCs and interpreted as the result of shock metamorphism (Heymann, 1967; Dodd, 1981; Kallemeyn et al., 1989; Stöffler et al., 1991). Based on the silicate darkening study in the CK4 chondrites Kobe and Karoonda, Tomeoka et al. (2001), Nakamuta et al. (2006), and Ohnishi et al. (2007) suggested that vesiculated olivines were formed by the crystallization of a melt produced by shock. Vesicles would be formed from volatiles initially present in matrix then in melt while inclusions would be the result of magnetite and sulfide melting droplets trapped in olivine. The conditions of the shock event were estimated at medium pressure $(<25 \mathrm{GPa})$ and high temperature $\left(>600^{\circ} \mathrm{C}\right)$ (Tomeoka et al., 2001).

\subsection{Refractory Inclusions in CK Chondrites}

By comparison with other groups of CCs, CK chondrites were described as CAI-poor meteorites (e.g., Kallemeyn et al., 1991; Keller et al., 1992; Rubin 2011), i.e., 0.2 area\% (Hezel et al., 2008) on the basis of literature data obtained for equilibrated CK chondrites (Noguchi, 
1993) and $~ 1$ vol\% regarding the CK-related, ungrouped type 3 CCs Ningqiang (Rubin et al., 1988; Kallemeyn et al., 1991). However, recent studies indicate that numerous CK3-4 chondrites contain between $\sim 10$ and 15 area\% of CAIs (Chaumard et al., 2009a, 2011, 2014). These results are in agreement with the high refractory lithophile abundances measured in CK chondrites (Kallemeyn et al., 1991), indicating that previous values of CAIs abundances in CK chondrites were thus underestimated and should be closer to those measured in CVs ( 10 vol. \%; e.g., Scott et al., 1996).

Rare earth element (REE) abundances measured in fine-grained CAI silicates display patterns similar to those of group I, II, and III (Chaumard et al., 2014), as defined from bulk refractory inclusions in CV chondrites (e.g., Grossman and Ganapathy, 1976). Both fine- and coarse-grained CAIs were observed in CK chondrites, with a mineralogy dominated by plagioclase, high-Ca pyroxene, spinel, magnetite, and olivine (e.g., Rubin et al., 1988; Keller et al., 1992; Noguchi, 1993; Chaumard et al., 2014).

In response to an increasing thermal metamorphism, petrographic observations of CAIs report Fe-enrichments in spinel and olivine and replacement of primary perovskite by ilmenite from unequilibrated CK3 chondrites (Geiger et al., 1993; Ivanova et al., 2000, Zipfel et al., 2000; Brandstätter et al., 2003; Smith and Russell, 2003) to higher metamorphic types (CK4/5) (MacPherson and Delaney, 1985; Keller, 1992; Keller et al., 1992; Greenwood et al., 2000a, 2000b, Kurat et al., 2002; Bukovanská et al., 2003).

In CK4, fine-grained CAIs recrystallized as irregular assemblages of plagioclase, high-Ca pyroxene, with minor amounts of olivine, low-Ca pyroxene, and magnetite (Chaumard et al., 2014). These fine-grained CAIs are mineralogically and chemically similar to the object composed of diopside, anorthite, and olivine described by Rubin (2013) in the CK3 NWA 1559 and interpreted as a metamorphosed amoeboid olivine inclusion (AOI). Although their recognition within a recrystallized matrix is difficult, AOIs were reported in the CK3 NWA 1559 by Brandstätter et al. (2003) and in CK4 chondrites (McSween, 1977; Keller et al., 1992).

Plagioclase, sometimes occurring as polysynthetically twinned crystals, is present as laths or anhedral grains, with sizes up to $\sim 500 \mu \mathrm{m}$ in size (e.g., Keller et al., 1992; Chaumard et al., 2014). While Keller et al. (1992) found nearly pure anorthite compositions, Rubin et al. (1988), Noguchi (1993), and Chaumard et al. (2014) reported significant compositional variations, from $\mathrm{An}_{15}$ to $\mathrm{An}_{100}$. Plagioclase contains significant amount of $\mathrm{MgO}(0.3-0.7 \mathrm{wt} \%)$ and up to $1.4 \mathrm{wt} \%$ $\mathrm{FeO}$ (Rubin et al., 1988; Chaumard et al., 2014).

Primary spinels are present as euhedral grains up to $200 \mu \mathrm{m}$ in size, enclosed in plagioclase, fassaite, or grossular. Sometimes close to the hercynite endmember, spinel compositions range from $\sim 5$ to $28 \mathrm{wt} \% \mathrm{FeO}$ and from $\sim 10$ to $25 \mathrm{wt} \% \mathrm{MgO}$, with significant amount of $\mathrm{NiO}$ (up to $\sim 2 \mathrm{wt} \%$ ), $\mathrm{TiO}_{2}$ (up to $\sim 1.8 \mathrm{wt} \%$ ) and $\mathrm{Cr}_{2} \mathrm{O}_{3}$ (up to $\sim 2.6 \mathrm{wt} \%$ ) (e.g., MacPherson and Delaney, 1985; Keller et al., 1992; Noguchi, 1993; Chaumard et al., 2014). Secondary Fe-rich spinels were also reported in coarse-grained CAIs by Chaumard et al. (2014).

High-Ca pyroxene is either present as chemically zoned laths (MgO-rich cores and $\mathrm{FeO}-$ rich borders) up to $\sim 350 \mu \mathrm{m}$ in size or as rounded grains (Noguchi, 1993; Chaumard et al., 2014). Compositions range from $\mathrm{Fs}_{9}$ to $\mathrm{Fs}_{26}$, with significant amount of $\mathrm{TiO}_{2}$ (up to $3.3 \mathrm{wt} \%$ ) and $\mathrm{Al}_{2} \mathrm{O}_{3}$ (up to $15 \mathrm{wt} \%$ in cores of compositionally zoned augite grains). Fassaite was also observed by Chaumard et al. (2014) in many CAIs from CK3 chondrites and contains 5.2-11.5 wt\% $\mathrm{TiO}_{2}, 15.8-21.9 \mathrm{wt} \% \mathrm{Al}_{2} \mathrm{O}_{3}, 6.6-11.0 \mathrm{wt} \% \mathrm{MgO}$. In coarse-grained CAIs, fassaite is partially destabilized into diopside and ilmenite (Chaumard et al., 2014). 
Extensively described in CAIs from CV chondrites (e.g., Hutcheon and Newton, 1981; Wark 1981, 1987; Hashimoto and Grossman 1987; Krot et al., 2004, 2007, 2009; Fagan et al., 2005, 2006, 2007), secondary grossular is less common in CAIs from CK chondrites. In these latters, secondary grossular is present as irregular masses surrounding primary anorthite ( $\sim \mathrm{Gr}_{89} \mathrm{Py}_{10}-\mathrm{Gr}_{99} \mathrm{Py}_{0}$ ) or as a fine-grained mixture (with anorthite) surrounding primary spinel and pyroxene ( $\left.\mathrm{Gr}_{73} \mathrm{Py}_{8}-\mathrm{Gr}_{99} \mathrm{Py}_{0}\right)$ (Noguchi, 1993; Chaumard et al., 2014). In coarse-grained CAIs, Chaumard et al. (2014) suggested a formation of secondary grossular by the reaction between primary anorthite and high-Ca pyroxene that imply formation of secondary Fe-rich spinel, or from the destabilization of primary melilite and anorthite in a two-stage process during fluidassisted thermal metamorphism as described in a compact type A CAI by Krot et al. (2009).

While abundant in CAIs in CV chondrites, melilite was only reported in a CAI from the CK3 DaG 431 (Smith and Russell, 2003). The "lack" of melilite in CAIs in CK chondrites can be interpreted as the consequence of its highly susceptibility to alteration (e.g., Allen et al. 1978; Wark 1981; McGuire and Hashimoto 1989; Nomura and Miyamoto 1998; Krot et al. 2007), as well as the fact that akermanite is not stable at high temperature (Harker and Tuttle, 1956; Yoder 1973).

\subsection{Opaque phases in CK Chondrites}

CK chondrites are highly oxidized meteorites (close to the Ni-NiO buffer; Geiger and Bischoff, 1995; Righter and Neff, 2007), as evidenced by the abundance of opaque phases that are dominated by Cr-rich ( 1.5-5 wt.\% $\mathrm{Cr}_{2} \mathrm{O}_{3}$; Greenwood et al., 2010a; Runyon and Dunn, 2011) magnetite (nearly pure $\mathrm{Fe}_{3} \mathrm{O}_{4}$ ) while metal is very rare or lacking (Kallemeyn et al., 1991; Geiger and Bischoff, 1991, 1995; Tachibana et al., 2002; Tomeoka et al., 2005; Neff and Righter, 2006; Righter and Neff, 2007; Greenwood et al., 2010a; Davidson et al., 2011, 2014c; Dunn et al., 2016). Magnetite in all CK chondrites typically contains between 0.2 and 0.4 wt.\% $\mathrm{NiO}$ (Greenwood et al., 2010a; Dunn, 2011; Dunn et al., 2016a). Magnetite in unequilibrated CK3 chondrites contains $\leq 0.1 \mathrm{wt} \% \mathrm{MnO}, 2.1-3.5$ wt. $\% \mathrm{Al}_{2} \mathrm{O}_{3}, \sim 0.5-1.0$ wt. $\% \mathrm{MgO}$, and $\sim 0.5-$ 1.2 wt.\% $\mathrm{TiO}_{2}$ (Runyon and Dunn, 2011; Davidson et al., 2014c; Dunn et al., 2016). In CK4-6 chondrites, magnetite typically contains small amounts of $\mathrm{Al}_{2} \mathrm{O}_{3}(\sim<2 \mathrm{wt} . \%), \mathrm{MgO}(<0.5$ wt.\%), $\mathrm{TiO}_{2}$ (<0.5 wt.\%) (e.g., Geiger and Bischoff; Greenwood et al., 2010a; Runyon and Dunn, 2011). Magnetite CK4-6 chondrites also exhibits lamellae of illmenite and aluminous spinel (e.g., Kallemeyn et al., 1991; Neff and Righter, 2006; Righter and Neff, 2007) that are interpreted as exsolutions formed during cooling from the peak metamorphic temperature (Geiger and Bischoff, 1990, 1995).

Opaque phases also consist of Fe and Fe,Ni sulfides. Although occurring in abundances lower than 1 vol.\% (Geiger and Bischoff, 1995), many phases were described. The most common sulfide phases are pentlandite $\left((\mathrm{Fe}, \mathrm{Ni})_{9} \mathrm{~S}_{8}\right)$, pyrite $\left(\mathrm{FeS}_{2}\right)$, monosulfide solid solution (MSS: (Fe,Ni) $\left.)_{1-\mathrm{x}} \mathrm{S}\right)$ ), and pyrrhotite $\left(\mathrm{Fe}_{1-\mathrm{x}} \mathrm{S}\right)$ (e.g., Geiger and Bischoff, 1995; Righter and Neff, 2006). Millerite $(\mathrm{NiS})$, chalcopyrite $\left(\mathrm{CuFeS}_{2}\right)$, violarite $\left(\mathrm{FeNi}_{2} \mathrm{~S}_{4}\right), \mathrm{PGE}$-rich phases such as $(\mathrm{Os}, \mathrm{Ru}, \mathrm{Ir}) \mathrm{S}_{2}$ (solid solution between laurite $(\mathrm{Ru}) \mathrm{S}_{2}$ and ehrlichmanite $\left.(\mathrm{Os}) \mathrm{S}_{2}\right)$, as well as telluride- and arsenide-rich phases were also reported in CK chondrites (Noguchi et al., 1993; Geiger and Bischoff, 1991, 1995; Huber et al., 2006). 


\subsection{Asteroidal Chemical and Mineralogical Processes Affecting the CK Chondrites}

\subsection{Models of Aqueous Alteration Processes Affecting the CK Chondrites}

Because of intense secondary metamorphism of most of the CK chondrite samples, only a few occurrences of parent body aqueous alteration features were reported so far. Aqueous alteration likely occurred during the first steps of the thermal metamorphic event that affected the CK parent body, hence the use below of the term 'thermal-assisted aqueous alteration'.

As reported for the CK-related Ningqiang chondrite, CV, R, and ordinary chondrites (e.g., Choi et al., 1997, 1998, 2000; Greenwood et al., 2000c; Choi and Wasson, 2003; Hsu et al., 2006), the formation of magnetite in CK chondrites has been attributed to parent body aqueous alteration processes (e.g., Righter and Neff, 2007; c et al., 2014). For example, magnetites from the anomalous CK3 Watson-002 and A-881595 that is supposed to be one of the least altered CK3 chondrite (Schrader et al., 2011), display oxygen isotope compositions supporting a formation by the oxidation of metal during thermal-assisted parent body aqueous alteration (Davidson et al., 2014c). Moreover, and as observed in the Ningqiang chondrite (Choi and Wasson, 2003), oxygen isotope compositions of some magnetite in the CK4 chondrites Karoonda and Y-7903 appear to have been reequilibrated on a very local scale (Clayton and Mayeda, 1999; Greenwood et al., 2000a,b; Hiyagon et al., 2001). Such observations are consistent with a very low water/rock ratio as experienced by $\mathrm{CV}_{\text {OxA }}$ (Greenwood et al., 2010a). In contrary, Davidson et al. (2014c) suggested that the CK3 Watson-002 and A-881595 might have experienced parent body aqueous alteration with higher water/rock ratios.

Additional evidences for a secondary aqueous alteration on the CK parent body were also reported by Chaumard et al. (2014) in a coarse-grained CAI from the CK4 Tnz 057. The authors described veins of secondary forsterite, Ca-rich pyroxene, and anorthite interpreted as the result of the dissolution of primary fassaite, spinel, and melilite, followed by in situ crystallization of these anhydrous phases from a high-temperature fluid.

\subsection{Oxidation state of CK chondrites}

As indicated by their high abundances of magnetite, the scarcity or lack of Fe,Ni metal, and the ubiquity of Ni-rich olivines, CK chondrites are highly oxidized meteorites (Kallemeyn et al., 1991; Noguchi, 1993; Geiger and Bischoff, 1995; Righter and Neff, 2007; Greenwood et al., 2010a). This oxidation state recorded the conditions that prevailed on the CK parent body (Greenwood et al., 2010a), likely during thermal-assisted aqueous alteration. The formation of magnetite and the presence Ni-rich olivines in $\mathrm{CK}$ chondrites require high oxygen fugacities, estimated close to the Ni-NiO buffer (from FMQ + 2 to FMQ + 4.5) (Geiger and Bischoff, 1995; Righter and Neff, 2007). Geiger et al. (1989) and Geiger and Bischoff (1995) performed annealing experiments on Allende chips under controlled oxygen fugacity conditions ( $\mathrm{Ni}-\mathrm{NiO}$ buffer) at $1050^{\circ} \mathrm{C}$ and $1150^{\circ} \mathrm{C}$ for four days. They reported the formation of oxide phases with high contents of $\mathrm{NiO}(1.9 \mathrm{wt} \%), \mathrm{TiO}_{2}(1.3 \mathrm{wt} \%), \mathrm{MgO}$ (3.4 wt $\%$ ), $\mathrm{Cr}_{2} \mathrm{O}_{3}(6.1 \mathrm{wt} \%)$, and $\mathrm{Al}_{2} \mathrm{O}_{3}$ 
(15.0 wt\%) and diffusion of $\mathrm{Ni}$ into olivine. By comparison with $\mathrm{CK}$ chondrites ( $\sim 0.3$ to 0.7 wt. $\% \mathrm{NiO})$, the higher $\mathrm{NiO}$ contents measured in these experiments is likely the consequence of the open system and non-equilibrium conditions. In agreement with experiments performed by Wulf et al. (1995), no sulfides were observed by Geiger et al. (1989) and Geiger and Bischoff (1995) because of the loss of S during these open system experiments, while CK chondrites contain Ni-rich sulfides.

\subsection{Thermal Metamorphism in CK Chondrites}

In addition to the low abundance of sulfides (e.g., Geiger and Bischoff, 1995), evidences for a metamorphic event under open system conditions are also given by the depletion of CK chondrites in Se (Kallemeyn et al., 1991; Geiger and Bischoff, 1989). Some other thermally labile elements such as $\mathrm{Cs}, \mathrm{Ga}, \mathrm{Ag}, \mathrm{Te}, \mathrm{Zn}$, and Bi were also lost during metamorphism (Kallemeyn et al., 1991; Wasson et al., 2013; Noronha and Friedrich, 2014), consistent with results from open-system heating experiments (Matza and Lipschutz, 1978).

Only few estimates of the peak metamorphic temperatures $\left(T_{\mathrm{p}}\right)$ were reported so far for CK chondrites. Based on the distribution of presolar components, Clayton et al. (1977) estimated a $T_{\mathrm{p}}$ of $\sim 525^{\circ} \mathrm{C}$ in the Karoonda CK4 chondrite, while Nakamuta et al. (2001) and Tachibana et al. (2002) reported a $T_{\mathrm{p}}$ of $\sim 700-800^{\circ} \mathrm{C}$ for the $\mathrm{CK} 4$ chondrite Kobe based on the chemical compositions of plagioclases and pyroxenes. Olivine-spinel thermometry and pyroxene compositions indicate that $\mathrm{CK} 4-5$ chondrites were heated at temperatures ranging from $\sim 750$ $850^{\circ} \mathrm{C}$ (Noguchi, 1993). The ${ }^{18} \mathrm{O} /{ }^{16} \mathrm{O}$ fractionations among major minerals in several $\mathrm{CK} 4$ chondrites suggest a narrow range of $T_{\mathrm{p}}$ between $590^{\circ} \mathrm{C}$ and $630^{\circ} \mathrm{C}$ (Clayton and Mayeda, 1999), which is consistent with results from Geiger and Bischoff (1991) that used the compositions of coexisting pyroxenes to estimate $T_{\mathrm{p}}$ in the range of $\sim 550-650^{\circ} \mathrm{C}$ for $\mathrm{CK} 4, \sim 650-800^{\circ} \mathrm{C}$ for $\mathrm{CK} 5$, and $\sim 800-1000^{\circ} \mathrm{C}$ for $\mathrm{CK} 6$. To a lesser extent, these results are also in good agreement with petrological and Al-Mg isotopic analyses in a coarse-grained CAI in the Tnz 057 CK4 chondrite indicating a $T_{\mathrm{p}}$ of $\sim 540-800^{\circ} \mathrm{C}$ (Chaumard et al., 2014). Using the Fe, Ti geothermobarometer based on the distribution of Ti between ilmenite and magnetite, Neff and Righter (2006) and Righter and Neff (2007) calculated a range of $T_{\mathrm{p}}$ between $280^{\circ} \mathrm{C}$ and $660^{\circ} \mathrm{C}$.

More recently, Chaumard and Devouard (2016) modeled Fe/Mg interdiffusion profiles measured in zoned olivines in chondrules from CK3-4 chondrites. The authors thus estimated both $T_{\mathrm{p}}$ and time scales of the thermal metamorphic event, from 50 to 70,000 years for $T_{\mathrm{p}}$ of $870^{\circ} \mathrm{C}$ and $650^{\circ} \mathrm{C}$, respectively. Both $T_{\mathrm{p}}$ and time scale were also estimated by Davidson et al. (2014c) for the anomalous CK3 Watson 002. The authors inferred a duration of $11 \times 10^{6}$ years using a $T_{\mathrm{p}}$ of $500^{\circ} \mathrm{C}$ corresponding to the lowest value determined for CO3.5 chondrites (e.g., Busemann et al., 2007), which would lead significant oxygen isotope exchange between magnetite and adjacent phases as observed in Watson 002. As discussed by Chaumard and Devouard (2016), the differences of $T_{\mathrm{p}}$ estimated for CK chondrites is likely the consequence of the use of various geothermometers that possibly recorded closure temperatures rather than peak metamorphic temperatures.

\subsection{Potential Relationship with CV Chondrites}


$\mathrm{CV}$ and $\mathrm{CK}$ chondrites display numerous similarities in terms of cosmic-ray exposure age distribution (both CV and CK chondrites show two clusters at $\sim 9 \mathrm{Ma}$ and $29 \mathrm{Ma}$; Scherer and Schultz, 2000), bulk elemental abundances, whole-rock oxygen isotope compositions, and mineralogical properties (e.g., Greenwood et al., 2003, 2004, 2010a; Devouard et al., 2006; Chaumard et al., 2009a, 2014, 2016; Wasson et al., 2013). These similarities strongly support a genetic relationship between $\mathrm{CV}$ and $\mathrm{CK}$ chondrites and thus a common asteroidal source, as previously implied by the establishment of the CV-CK clan (Weisberg et al., 2006). The differences observed between $\mathrm{CV}_{\mathrm{R}}, \mathrm{CV}_{\mathrm{OxA}}, \mathrm{CV}_{\mathrm{OxB}}$, and $\mathrm{CK}$ chondrites are likely the consequence of different intensities of parent body aqueous alteration and thermal metamorphism. Within this framework, $\mathrm{CV}_{\mathrm{OxA}}, \mathrm{CV}_{\mathrm{OxB}}$, and $\mathrm{CK}$ chondrites probably formed from a $\mathrm{CV}_{\mathrm{R}}$-like chondrite precursor by oxidation caused by aqueous alteration then thermal metamorphism (e.g., Greenwood et al., 2010a; Wasson et al., 2013). Thus, rather than two distinct groups of carbonaceous chondrites, CV and CK chondrites might form one single and continuous metamorphic series from the petrologic types 3 to 6 . In consequence, CV and CK most probably derived from a common asteroid, or at least from similar parent bodies formed contemporaneously in the same region of the protoplanetary disk.

On the basis of these results, different parent body models and scenario for the asteroidal source of the CV and CK chondrites can be considered. Greenwood et al. (2010a) proposed a single thermally parent body, as it has been postulated for ordinary chondrites (e.g., Trieloff et al., 2003; Wood, 2003). In this model, the most metamorphosed lithologies (CK chondrites) and the less heated ones (CV chondrites) are located in the core and at the surface of the parent body, respectively.

Recent paleomagnetic studies suggest that CV chondrites could derive from the external part of a differentiated asteroid with a molten core (e.g., Weiss et al., 2010; Carporzen et al., 2011; Elkins-Tanton et al., 2011). In this model, the CK chondrites would be located in the lower part of the undifferentiated chondritic layer situated at the surface of the differentiated asteroid.

As discussed by Wasson et al. (2013), such model was challenged by Bland et al. (2011) who suggested that impacts on a CV material could explain their magnetic properties. Metamorphic reheating caused by impacts was discussed by Rubin $(1995,2004)$ and recently proposed by Wasson et al. (2013) to account for observations interpreted as shock features and thus evidences for impacts. CK may have been formed from a CV-type material that was shocked, buried, affected by aqueous alteration, then annealed. However, although shock features were recognized in CK chondrites (e.g., Kallemeyn et al., 1991; Wasson et al., 2013), there is no correlation between petrologic types and shock stages for CK chondrites, in a sense that the most metamorphosed CK chondrites do not seem to correspond to the most shocked samples (Rubin, 1991, 1992; Scott et al., 1992). To account for the lack of correlation between petrologic types and shock stages, Rubin (1992) suggested that CKs were shocked prior to be annealed whereas Tomeoka et al. (2001) proposed that the shock event occurred on a preheated parent body $\left(>600^{\circ} \mathrm{C}\right)$ at low pressure $(<25 \mathrm{GPa})$.

Chaumard et al. $(2009 \mathrm{~b}, 2012)$ proposed another parent body model based on the radiative heating of meteoroids during close approaches to the Sun (up to $\sim 780^{\circ} \mathrm{C}$ at perihelia $<0.15 \mathrm{AU})$. In this model, the intensity of this secondary thermal process depends on the size of the meteoroids, durations for which orbits are kept stable at small perihelia, and/or heliocentric distances. Such meteoroids could have been produced by the breakup of an initially homogeneous CV-type near-Earth object, thus forming such a swarm as also initially proposed 
by Kallemeyn et al. (1991) on the basis of the ubiquity and high abundance of CK meteorites with small sizes in Antarctica.

Although slightly different in term of their internal structures, CV-CK parent body models invoking a differentiated or partially differentiated asteroid appear to be at odd with the time and durations estimated for the CV-CK metamorphic event (e.g., Chaumard and Devouard, 2016). Indeed, such models of a thermally stratified asteroid imply an internal source of heat (decay of ${ }^{26} \mathrm{Al}$ ) and thus durations in the order of 1-100 Ma (e.g., Bennett and McSween, 1996; Ghosh and McSween, 1998; McSween et al., 2002; Huss et al., 2006; Henke et al., 2013) that are not consistent with the transient high-temperature features observed in CK chondrites (e.g., Wasson et al., 2013; Chaumard and Devouard, 2016). Although still debated, radiative and impact-related heating seem to be the most viable sources of heat to explain the textural and petrologic features of the CK chondrites.

Objections against a genetic link and thus a common parent asteroid for CV and CK chondrite are the lack of breccias composed of $\mathrm{CV}$ - and CK-like materials, as well as polymict breccias containing CK lithologies with different petrologic types as observed for ordinary and Rumuruti chondrites (Bischoff et al., 2006). In addition, some caveats have been also pointed out about a common parent CV-CK parent asteroid. For example, the distinct chromium isotope compositions of $\mathrm{CV}$ and $\mathrm{CK}$ chondrites may not support a common parent body (Yin et al., 2017). Moreover, Dunn et al. (2016a) and Dunn and Gross (2017) reported distinct chemical compositions (e.g., $\mathrm{Cr}_{2} \mathrm{O}_{3}, \mathrm{NiO}, \mathrm{TiO}_{2}$ ) of magnetite in the $\mathrm{CV}$ and $\mathrm{CK}$ chondrites, and a relationship of these compositions between $\mathrm{CV}$, unequilibrated $\mathrm{CK}$, and equilibrated $\mathrm{CK}$ that seem to be hardly compatible with a formation of CK chondrites from a CV-type precursor. As also noticed by Dunn et al. (2016a), the increase of the oxygen fugacity from CV to CK chondrites and the origin of the oxidizing agent seem to be not easily reconcilable with a common CV-CK parent body model.

\subsection{Implications for exploration of the CK parent body}

CCs derived from parent asteroids mostly located in the outer part of the asteroid belt (e.g., Gradie and Tedesco, 1982; Ghosh et al., 2006). However, since CV and CK chondrites are found on Earth, their orbits before encountering the Earth were those of near-Earth objects (NEOs). A combination of close encounters with the terrestrial planets and resonances with the giant planets can modify the osculating elements of NEOs during time (e.g., Williams and $\mathrm{Wu}$, 1993; Morbidelli et al., 2002; Michel et al., 2005). While a significant number of NEOs end their orbital evolution colliding with the Sun (e.g., Farinella et al., 1994), several NEOs with low perihelion distances $(<0.1 \mathrm{AU})$ can keep stable orbits for up to several million years (Marchi et al., 2009; Michel and Delbo, 2010).

Based on similar infrared and visible spectra, Bell (1998) first suggested that the Eos family members (termed $\mathrm{K}$ class asteroids) match to the $\mathrm{CV}$ and $\mathrm{CO}$ chondrites. However, more recent analyses indicate a closer match of most of the Eos family members to $\mathrm{R}$ and $\mathrm{CK}$ chondrites (two highly oxidized group of chondrites), while CV chondrites can match for some of the other members (Mothé-Diniz et al., 2008). These results are in good agreement with laboratory experiments showing that the mid-IR spectra of CK4-5 chondrites are similar to those of $\mathrm{CV}$ chondrites, both displaying a maximum of absorption around $11.3 \mu \mathrm{m}$ indicative of the high abundance of olivine in these samples (Beck et al., 2014). Moreover, in addition to the 1.05 
$\mu \mathrm{m}$ absorption band of olivine, many $\mathrm{CK}$ chondrites spectra are also blue-sloped beyond $1.5 \mu \mathrm{m}$, similarly to the Eos family (Cloutis et al., 2012).

Because the Eos family is located in the outer asteroid belt (2.95-3.13 AU; Vokrouhlický et al., 2006; Mothé-Diniz et al., 2008) that is dominated by C class asteroids (Gradie and Tedesco, 1982), CK chondrites more likely match to these objects than R chondrites (Greenwood et al., 2010a,b). The disrupted asteroid at the origin of the Eos family is thus a plausible source of the $\mathrm{CV}$ and $\mathrm{CK}$ meteorites and might be a potential future target for the exploration of primitive asteroids by spacecraft.

\subsection{Terrestrial Chemical and Mineralogical Processes Affecting the CK Chondrites}

Some CK chondrites (e.g., DaG 431, LEW 86258) found in hot and cold deserts display low abundances of siderophile elements such as $\mathrm{Ni}, \mathrm{Co}, \mathrm{Au}$, and Se (Kallemeyn et al., 1991; Huber et al., 2006). These observations are interpreted as the result of the destabilization by oxidation of sulfides (mostly pentlandite) to sulfates during terrestrial weathering, these latters being sometimes removed from the samples by leaching (Huber et al., 2006). Moreover, a few products of terrestrial weathering such as limonite and barite $\left(\mathrm{BaSO}_{4}\right)$ were observed also in NWA 1563 and NWA 060, respectively (Hubert et al., 2006)

Since CK chondrites as highly oxidized, Rubin and Huber (2005) proposed a new weathering index (wi) for CK chondrites. This index is mainly based on the modal abundance of brown-stained crystalline material caused by the mobilization of oxidized iron deriving from the terrestrial weathering of sulfides (Rubin and Huber, 2005). Modal abundances can be estimated from microscopic observations of thin sections in transmitted light, from $<5 \mathrm{vol} \%$ (wi-0) to wi-6 (significant weathering replacement of mafic silicates by phyllosilicates).

\subsubsection{Chemical and Mineralogical Processes Affecting the $\mathrm{CB}$ and $\mathrm{CH}$ Chondrites}

\subsubsection{Physical Properties of $C B$ and $C H$ chondrites}

The physical properties of CB chondrites are significantly different from other carbonaceous chondrites, as it would be expected from their extremely high metal contents. Macke et al. (2011) studied the physical properties of two $\mathrm{CB}$ chondrites $\left(\mathrm{CB}_{\mathrm{a}}\right.$ Bencubbin and $\mathrm{CB}_{\mathrm{b}} \mathrm{H}$. al Hamra 237), which they found to have similar properties. They determined the average bulk density of CB chondrites to be $5.25 \mathrm{~g} / \mathrm{cm}^{3}$ (ranging from $4.90-5.55 \mathrm{~g} / \mathrm{cm}^{3}$ ) and the grain density $5.65 \mathrm{~g} / \mathrm{cm}^{3}$ (ranging from $5.63-5.66 \mathrm{~g} / \mathrm{cm}^{3}$ ). For the CB chondrites, Rochette et al. (2008) measured the average magnetization to be $\log \chi=5.65 \pm 0.04$. Macke et al. (2011) obtained values that are within error of the results from Rochette et al. (2008), $\log \chi=5.57$ (range from 5.31 to $5.79 ; \log \chi *$ in $\log 10^{-9} \mathrm{~m}^{3} / \mathrm{kg}$ ). As expected, these are among the highest magnetizations for any chondrite group.

The physical properties of $\mathrm{CH}$ chondrites are closer to those of other carbonaceous chondrites. Macke et al. (2011) analyzed one $\mathrm{CH}$ chondrite, Acfer 214, having a bulk density $3.77 \mathrm{~g} / \mathrm{cm}^{3}$, and grain density $3.65 \pm 0.02 \mathrm{~g} / \mathrm{cm}^{3}$, and magnetization $\log \chi=5.30 \pm 0.12$. 
Rochette et al. (2008) also measured the magnetization of four $\mathrm{CH}$ chondrites (Acfer 182-207214, ALH 85085, NWA 739, and PCA 91328), which was withing error of the measurement from Macke et al. (2011), $\log \chi=5.36 \pm 0.20$.

\subsubsection{Chemical and Mineralogical Characteristics of $\mathrm{CB}$ and $\mathrm{CH}$ chondrites}

Bencubbin-like meteorites (CB) are metal-rich (up to 60 vol\%) chondritic breccias (Weisberg et al., 2001). they consist of Fe,Ni metal, chondrules, CAIs, AOAs, and sometimes hydrated lithic clasts. The CB chondrites are further divided into the CBa subgroup, characterized by large metal globules and chondrules, and the more metal-rich CBb subgroup with smaller chondrules and metal globules (Krot et al., 2001). Petaev et al. (2001) proposed that the zoned metal in CBbs could have formed in a locally very dusty (10-40 times typical) region of the nebula. Campbell et al. (2002) and Krot et al. (2005) suggested that both the metal and the low iron barred olivine (BO) and CC chondrules in the CBas condensed from an impact plume generated by an impact between a metal-rich body and another containing low iron silicates. Krot et al. (2005) subsequently argued that all $\mathrm{CBa}$ and $\mathrm{CBb}$ components formed in a giant impact: some through melting and the remainder by gas-solid condensation from an impact derived gas, $\sim 5$ my after CAI condensation. However, this model does not explain all aspects of CB mineralogy (Tang and Dauphas, 2012).

$\mathrm{CH}$ chondrites (Bischoff et al. 1993) appear related to CBs in terms of high metal abundance, and basically share the same minerlogy, but weith differing modal amounts of CAI, AOS, chondrules and chondrule sizes. Wasson and Kallemeyn (1990) argued that ALH 85085 $(\mathrm{CH})$ formed by condensation from an impact-generated vapor cloud, and that the high metalsilicate ratios were the result of subsequent regolith processes after reaccretion of the impact debris into a new steroid, which is similar to the impact model for the possibly related CBs.

Some CB and $\mathrm{CH}$ chondrites contain fine-grained, phyllosilicate-bearing, hydrated lithic clasts (Greshake et al., 2002). These clasts contain no anhydrous silicates and consist of framboidal and plaquette magnetite, Fe-Ni sulfides (pentlandite and pyrrhotite), and Fe-Mn-Mg$\mathrm{Ca}$-carbonates set in a phyllosilicate-rich matrix. Serpentine and saponite have been identified among the phyllosilicates. The absence of aqueous alteration of chondrules and metal grains in $\mathrm{CB}$ and $\mathrm{CH}$ chondrites indicates that the clasts experienced hydration in an asteroidal setting prior to incorporation into the $\mathrm{CH}$ and $\mathrm{CB}$ parent bodies. The hydrated clasts were either incorporated during regolith gardening or accreted together with chondrules and FeNi-metal grains after these high-temperature components had been transported from their hot formation region to a much colder region of the solar nebula. These clasts require mich additional characterization before they can be properly understood. At present they dop not appear to correspond to any existing meteorite class or type.

The meteorite Isheyevo is special in several respects (Ivanova et al,., 2008). It is a metalrich carbonaceous chondrite that contains several lithologies with different abundances of Fe,Ni metal (7-90 vol\%), with the metal-rich lithologies (50-60 vol\% of Fe,Ni metal) being dominant. The meteorite has aspects of both the $\mathrm{CB}_{\mathrm{b}}$ and $\mathrm{CH}$ carbonaceous chondrites, inclusing basic mineralogy, and bulk chemical and oxygen isotopic compositions, strengthening the apparent link between these groups. Bulk nitrogen isotopic composition is highly enriched in ${ }^{15} \mathrm{~N}\left(\delta^{15} \mathrm{~N}=\right.$ $1122 \%$ ). Similar to $\mathrm{CB}_{\mathrm{b}}$ chondrites, $\sim 20 \%$ of Fe,Ni-metal grains in Isheyevo are chemically zoned. Similar to $\mathrm{CH}$ chondrites, some metal grains are Ni-rich (>20 wt\% Ni). In contrast to $\mathrm{CB}_{\mathrm{b}}$ and $\mathrm{CH}$ chondrites, most metal grains are thermally decomposed into Ni-rich and Ni-poor 
phases. Similar to $\mathrm{CH}$ chondrites, chondrules have porphyritic and non-porphyritic textures and ferromagnesian (type I and II), silica-rich, and aluminum-rich bulk compositions. Some of the layered ferromagnesian chondrules are surrounded by ferrous olivine or phyllosilicate rims. Phyllosilicates in chondrule rims are compositionally distinct from those in the hydrated lithic clasts. Similarly to $\mathrm{CH}$ chondrites, CAIs are dominated by the hibonite-, grossite-, and meliliterich types; AOAs are very rare. The meteorite has experienced a high degree of shock metamorphism. It is clear that Isheyevo is a complex mixture of materials formed by different processes and physico-chemical conditions. There are also proiinent lamination to the meteorite's structure suggestive of sedimentation - Garvie et al. (2017) report dish structures.

Moyano-Cambero et al. (2016) have proposed a link between asteroid 21 Lutetia and the CH chondrites, especially PCA 91467.

\subsubsection{Physical Properties, Chemical, and Mineralogical Processes Affecting Unique Carbonaceous Chondrites Relevant to Space Exploration}

There are numerous, fascinating, unique carbonaceous chondrites, precluding a comprehensive review of all these samples within the constraints of this volume. Two of those chondrites, Tagish Lake and Kaidun, are described in detail because of their relevance to space exploration.

\subsubsection{Tagish Lake}

\subsection{Recovery of Tagish Lake}

The fireball producing the Tagish Lake meteorite occurred at local dawn on 18 January 2000. This exceptionally bright fireball was witnessed throughout the Yukon Territory, northern British Columbia, and parts of Alaska. This was the largest fireball to enter earth's atmosphere in 2000, and the largest over dry land in a decade (Brown et al., 2000).

The calculated orbit for Tagish Lake is a typical Earth-crossing Apollo asteroid-type orbit with a semimajor axis in the middle of the asteroid belt. A dynamical linkage with outer-belt objects is possible as the IR spectra of bulk Tagish Lake are most similar D-type asteroids (Hiroi et al., 2001). The orbit also crosses much of the main belt at low inclination, which suggests that the source of the carbonaceous chondrites responsible for the xenoliths found in some meteorite breccias might be similar to the source of the Tagish Lake meteorite.

Local resident Jim Brook recovered the first specimens from frozen surface of Tagish Lake on 25 January 2000. He continued collecting material on 26 January and was able to gather several dozen pieces in all, totaling $\sim 0.85 \mathrm{~kg}$. These fragments were collected from the surface of the frozen lake with ambient temperatures having remained subzero, and were placed in plastic bags without being handled by bare hands. This rapid recovery minimized contamination and, combined with the frozen state of the meteorites, may permit characterization of the full range of volatile organics in a primitive chondrite (Pizzarello et al., 2001; Herd et al., 2016). Additional specimens (the bulk of the collected mass) were recovered the following spring, and these later samples were all contaminated by lake water, changing mineralogy and physical properties to varying degrees (Zolensky et al., 2002).

\subsection{Physical Properties of Tagish Lake}

Ralchenko et al. (2014) measured the porosity (average $\sim 29 \%$ ), bulk density (average $\sim 1.8 \pm$ $0.03 \mathrm{~g} / \mathrm{cm}^{3}$ ) and grain density (average $\sim 2.6 \mathrm{~g} / \mathrm{cm}^{3}$ ) for frozen fragments of Tagish Lake. 
Rochette et al. (2008) measured the magnetization of Tagish Lake to be $\log \chi=4.94 \pm 0.04$ (in $10^{-9} \mathrm{~m}^{3} / \mathrm{kg}$ ).

\subsection{Classification of Tagish Lake and Affinities with Other Carbonaceous Chondrites}

The classification of Tagish Lake is complex (Zolensky et al., 2002). Tagish Lake is a brecciated, matrix-supported mixture of olivine-rich aggregates, sparse chondrules (diameter $<1$ $\mathrm{mm}$, about 1-2 per thin section), altered calcium-aluminum-rich inclusions (CAIs) up to $2 \mathrm{~mm}$ in diameter, magnetite (mainly framboids and plaquettes), individual grains of olivine, $\mathrm{Ca}-\mathrm{Mg}-\mathrm{Fe}-$ Mn carbonates, and Fe-Ni sulfides including pyrrhotite. See Figure TL1. Some chondrules and the majority of aggregates show evidence of aqueous alteration. The CAIs have the sinuous textures typical of CMs but are almost completely altered to phyllosilicates, predominantly $\mathrm{Mg}$ rich serpentine. In contrast, altered CAIs in CM chondrites usually consist of Fe-rich serpentine and diopside. Sulfides (in particular the coarse-grained sulfides present in CIs and all of the metamorphosed carbonaceous chondrites) are less abundant than in most CM or CI chondrites, and magnetite is far more abundant than in most CMs. In many places, magnetite has partially or completely replaced sulfides. Carbonates in Tagish Lake vary in composition from calcite $\left(\mathrm{CaCO}_{3}\right)$ to siderite $\left(\mathrm{FeCO}_{3}\right)$ and magnesite $\left(\mathrm{MgCO}_{3}\right)$. These $\mathrm{Mg}$ - and Fe-rich compositions have not been reported in any other carbonaceous chondrites. Some carbonates have Mn contents up to $1 \mathrm{wt} \%$. Olivine has the compositional range $\mathrm{Fa}_{0-29}$ with a peak at $\mathrm{Fa}_{1}$; pyroxene is $\mathrm{Fs}_{1-7}$ with a peak at Fs2. The meteorite shock stage is S1, which is typical for carbonaceous chondrites. The matrix consists mainly of phyllosilicates, mainly intergrown serpentine and saponite. The meteorite contains several distinct lithologies, including the carbonate-rich and carbonate-poor main lithologies, and occasional CM1 material (Zolensky et al., 2002).

The oxygen isotopic composition of bulk Tagish Lake is similar to those of CI chondrites and of a group of metamorphosed carbonaceous chondrites (such as Belgica 7904, Yamato 82162, and Yamato 86720 (Zolensky et al., 2002). If the data on Tagish Lake are interpreted in terms of the model of exchange of minerals with liquid water on a parent body, assuming equilibrium between phyllosilicates and carbonates, the oxygen isotopic data suggest a higher water/rock ratio than the $\mathrm{CM}$ group, and a lower temperature of aqueous alteration (near $0^{\circ} \mathrm{C}$ ) than the CI group.

Carbon concentration and isotopic composition were determined to be 5.4 wt \%, with summed $\delta^{13} \mathrm{C} \sim+24.3 \%$, respectively. Around $3.7 \mathrm{wt} \%$ carbon derives from carbonates, a much higher proportion than is usual for either CI or CM chondrites, where 0.2 to $0.5 \mathrm{wt} \%$ carbon typically occurs as carbonate; the carbon isotopic composition of the carbonate, however, is within the range of values for $\mathrm{CI}$ and $\mathrm{CM}$ chondrites $(+20$ to $+70 \%$ ). The remaining carbon is mostly from organic species that combust below $\sim 500^{\circ} \mathrm{C}$. The $\delta^{13} \mathrm{C}$ of the organics varies from 10 to $-1 \%$, a range similar to that observed for organic species in CI and CM meteorites (Zolensky et al., 2002). Tagish lake was one of the first chondrites in which organic nanoglobules were reported (Nakamura et al., 2002, 2006). 


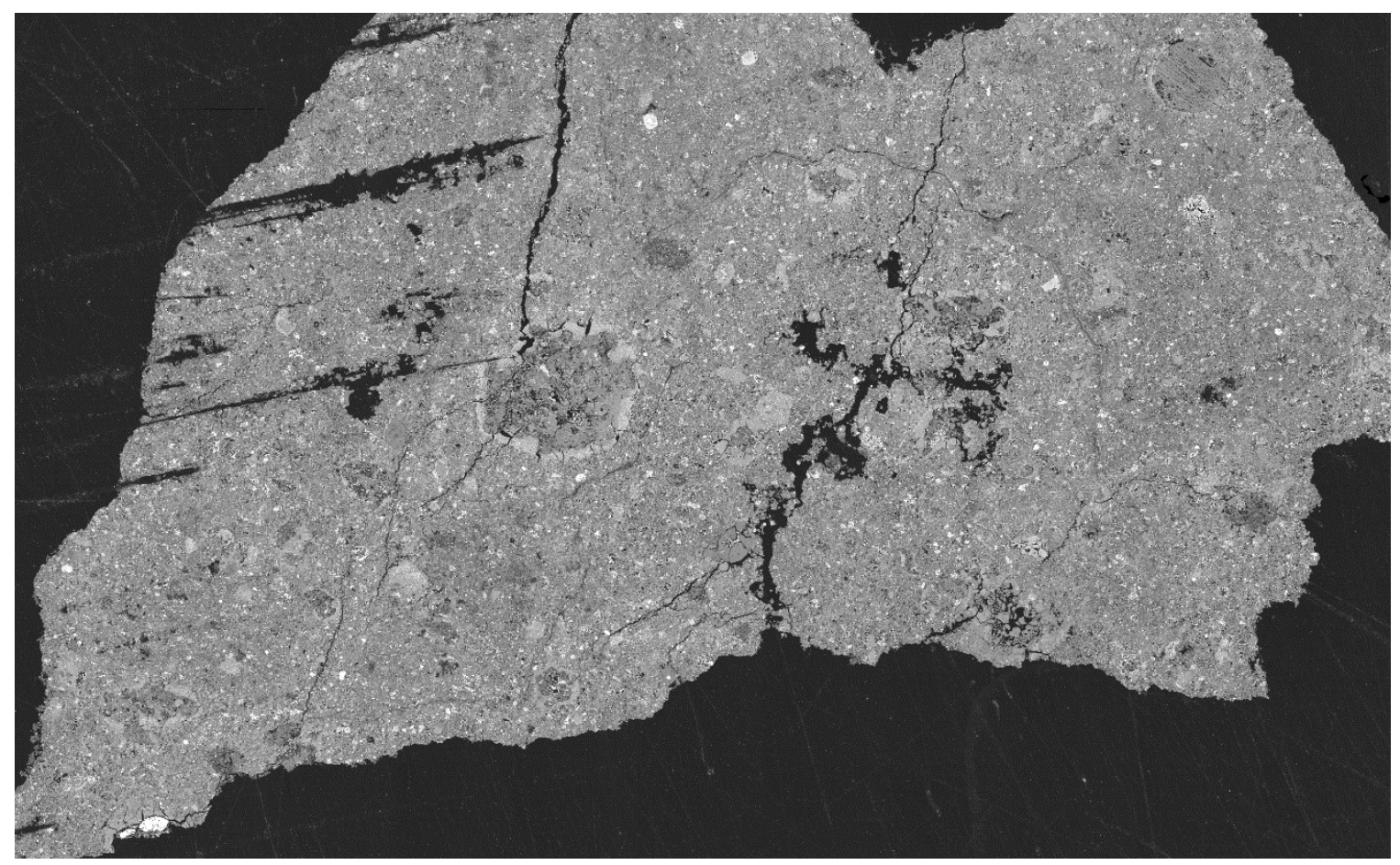

Figure TL1. BSE image of Tagish Lake, showing the paucity of chondrules, with irregular phyllosilicate rims. The majority of the image is phyllosilicates. All white objects are Fe-Ni sulfides. Image is $2 \mathrm{~cm}$ across.

\subsubsection{Kaidun}

\subsection{Physical Properties of Kaidun}

Little is known about the physical properties of Kaidun and these properties are likely to vary significantly from sample to sample. Rochette et al. (2008) measured the magnetization of Kaidun to be $\log \chi=4.69 \pm 0.08$ (in $10^{-9} \mathrm{~m}^{3} / \mathrm{kg}$ ).

\subsection{Chemical and Mineralogical Characterisitcs of Kaidun}

The complex Kaidun microbrecccia meteorite contains an unprecedented accumulation of materials from many different asteroids, principally carbonaceous and enstatite chondrites, but also many other frequently strange materials (Zolensky and Ivanov, 2003). The following wellknown meteorite types are definitely present in Kaidun: EH3-5, EL3, CV3, CM1-2, brachinites, ordinary chondrites, and R chondrites. Also present in Kaidun are new C1 and C2 type lithologies, unique alkaline-enriched clasts, impact melt products, phosphide-bearing clasts, vein- and cavity-filling materials, new enstatite-bearing clasts, and Ca-rich achondrite materials. Many further materials have yet to be characterized. Obviously, the Kaidun parent object accumulated materials from across the entire main asteroid belt. Many of these materials were subjected to varying levels of physical processing, heating, shock, melting, and aqueous alteration. Kaidun is important because it contains many asteroidal materials we have not seen before, providing a more complete view of the diversity of materials in the asteroid belt than has 
been provided by other meteorites. This is possible because of the small, generally submillimeter- size of the component clasts in Kaidun - it is far easier for these smaller objects to scatter throughout the solar system than it is for larger, conventionally-sized meteorites.

In this chapter we describe only a few of the dominant, clearly-identified Kaidun lithologies. For a more complete report see Zolensky and Ivanov (2003).

Enstatite (usually En >98) is the principal mineral in the EH3 lithology, of course. Plagioclase or glass of plagioclase composition $(\mathrm{Ab}>95)$ fills small interstices between enstatite grains in chondrules and is found in the matrix. Silica is usually present as small grains together with plagioclase. Fe-Ni metal forms large aggregates of different types and abundances in the matrix. Metal is rather similar in all lithologies in Ni contents (5.5-5.8 wt.\%) but with varying $\mathrm{Si}$ contents (2.8 to $3.3 \mathrm{wt} . \%)$. Rare metal grains in chondrules are poorer in Si (1.6-2.1 wt.\%). Schreibersite is usually present in the matrix in association with kamacite. It is rich in Ni (18.819.7 wt.\%) in all lithologies. Troilite is the dominant sulfide. It typically has relatively constant contents of $\mathrm{Mn}(<0.1 \mathrm{wt} . \%)$, Ti $(\sim 0.35 \mathrm{wt} . \%)$ and $\mathrm{Cr}(0.8-1.0 \mathrm{wt} . \%)$. Niningerite is also a typical matrix mineral. Its composition is similar in all Kaidun EH lithologies and shows high Mn contents (10.3-11.8 wt.\%) that are characteristic of the most common group of EH niningerites (Ehlers and El Goresy 1988). A particularly interesting feature is that hydrated phases are abundant in all EH lithologies in Kaidun, in contrast to all other examples of EH chondrites which are entirely anhydrous. The phyllosilicates in wet Kaidun E chondrite lithologies s are discussed elsewhere in this chapter in the section on Enstatite Chondrites.

The EL3 lithology has a clear unequilibrated texture - well-defined chondrules set within a fine-grained matrix. The chondrules vary from $<0.3$ up to $6 \mathrm{~mm}$ in size, and their fragments and large (up to $1 \mathrm{~mm}$ ) xenomorphic metal-sulfide-schreibersite intergrowths are set within matrix consisting of black opaque material containing grains of enstatite, olivine, kamacite and troilite. The chondrules are sharp and well-defined, typical of type 3 chondrites, and comprise about 45 vol.\% of the lithology. Enstatite-rich radial chondrules are the most abundant type. Also present are olivine-pyroxene microporphyritic chondrules with idiomorphic olivine grains in the center, and enstatite-rich barred chondrules. Ferromagnesian glass of variable composition, albitic glass, silica and diopside are present in the chondrules as rare accessory phases. The opaque minerals are represented by Fe-Ni metal, Fe-Ni sulfides and schreibersite.

Carbonaceous chondrite lithologies appear to dominate Kaidun. Some of these, such as the CV3 and CM1 lithologies, are easily recognized materials. However, there are also numerous type 2 and 1 materials which do not appear to have analogues in other meteorites. The bulk of the CV3 lithology in Kaidun consists of chondrules, chondrule fragments, olivine-rich aggregates, lithic fragments and a sulfide aggregate, set within finer-grained, olivine-rich matrix. Matrix consists of $15 \mu$ - to sub-micron size olivine (Fa52-41), low-Ca pyroxene, and pyrrhotite. This Kaidun CM2 lithology consists of matrix of fine-grained serpentine and pyrrhotite. Set within this matrix are submillimeter-sized chondrules, all of which are incompletely altered to serpentine and pyrrhotite. ThCM1 lithology is very abundant in Kaidun, and is found in most Kaidun thin sections. The bulk of this Kaidun lithology consists of fine-grained serpentine, saponite, and minor clinochlore. Floating in the phyllosilicate-dominated matrix are abundant acicular, mantled pyrrhotites (a very distinctive feature), framboidal magnetite, pentlandite, hydroxyl-apatite, and rare diopside grains. Set within this matrix are submillimeter-sized complex aggregates containing phyllosilicates, sulfides and melanite garnet, and abundant, homogeneous lumps of phyllosilicates. Some samples contain phyllosilicate/carbonate veins. 
Zolensky and Ivanov (2003) suggested that the final parent object where Kaidun was assembled was a large C-type asteroid, but a "wet" M- or E-type is also possible.

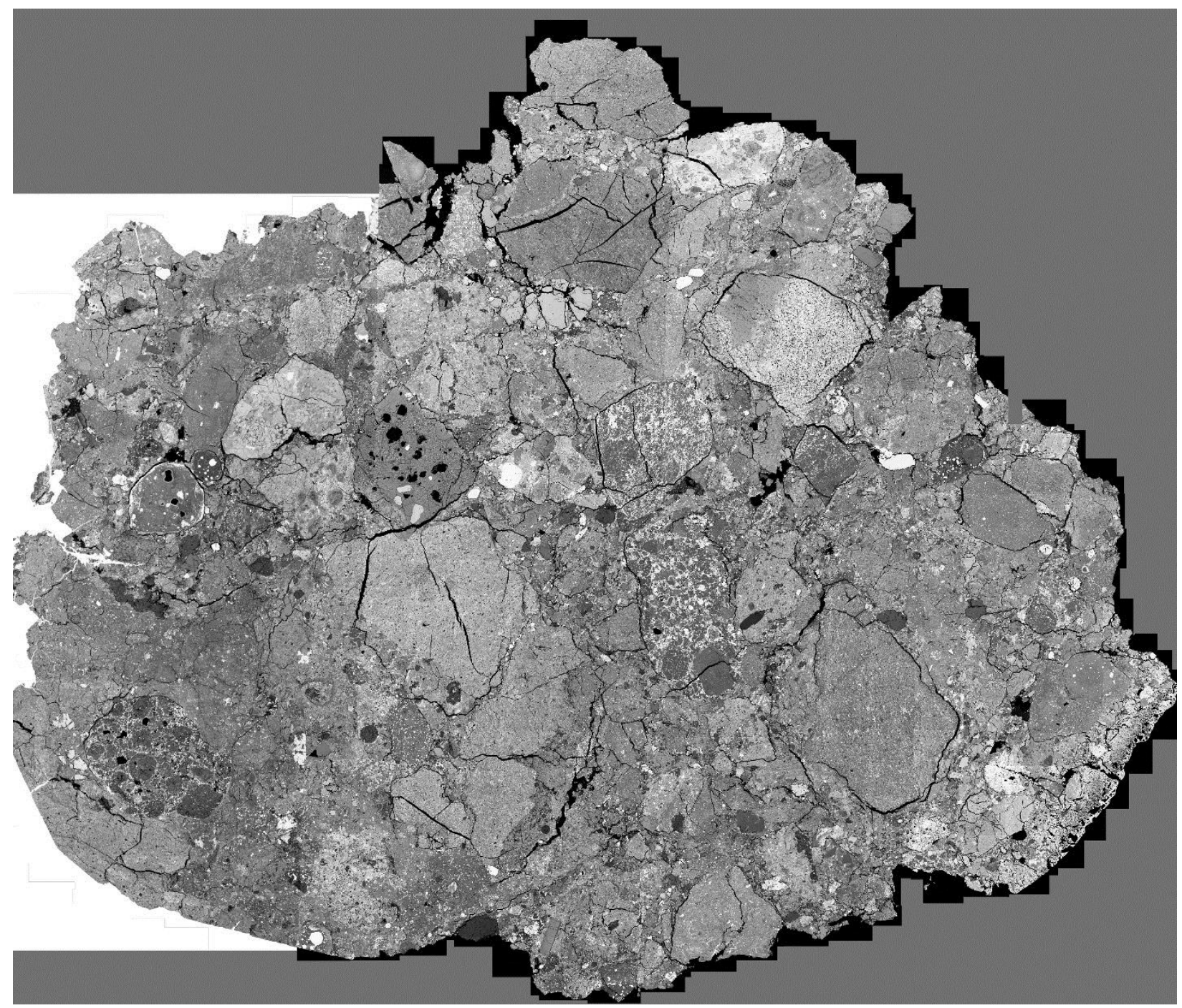

Fig. K1. BSE image of a thin section of Kaidun, showing many different lithologies, in cm to $\mathrm{mm}$ sized clasts. Image measures $5 \mathrm{~cm}$ across.

\subsection{Insolation Thermal Metamorphic of Hydrated Carbonaceous Chondrites.}

The meteorites that best match the spectra of current sample-return-mission target asteroids include thermally anomalous $\mathrm{C}$ chondrites and experimentally heated CM2s (e.g., Clark et al., 2011). Some CM chondrites have mineral inventories and attributes that indicate they were dehydrated during heating to peak temperatures above those of typical hydrated CCs (e.g., Tonui et al., 2002, 2003, 2014; Table 2.6). Spectral reflectance data in the ultraviolet (UV), visible and near infrared (IR) for thermally metamorphosed carbonaceous chondrites (e.g., Tonui et al., 2014) suggest that carbonaceous chondrite-like material that has been metamorphosed over a range of temperatures now occurs at the surface of a number of C-, G-, B-, and F- asteroids 
(Hiroi et al., 1993, 1994, 1996). Might such thermally metamorphosed material have formed at these asteroids' surfaces?

The orbits of sample-return mission-target asteroids 25143 Itokawa, 162173 Ryugu, and 101955 Bennu all have perihelion distances of 0.88 - 0.96 AU (REFS). The pre-atmospheric orbits of witnessed and recovered meteorite falls mostly have perihelion distances $\sim 0.9 \mathrm{AU}$ (mean of perihelion distances calculated from orbital elements compiled by Brown et al., 2011). At these heliocentric distances in the present epoch, neither category of objects experiences insolation heating above that experienced by the Earth and Moon at 1 AU. However, over the orbital lifetimes of asteroids and meteoroids, there exists a finite probability that many of them were in orbits with perihelia closer to the sun (Marchi et al., 2009). During orbital passes near the Sun ( $<1 \mathrm{AU}$ ), NEOs (including PHOs and AAs) have been exposed to insolation heating to the extent that their surfaces have been heated to temperatures well above the ambient temperature of their source bodies (Marchi et al., 2009), which are ultimately mostly Main Belt asteroids. Surfaces of NEOs with perihelia $<0.5 \mathrm{AU}$ or $<0.1 \mathrm{AU}$ may have been heated to temperatures as high as 400-600K or 1200-2000K, respectively (Marchi et al., 2009). These potentially

accessible surface temperatures include the range of peak metamorphic temperatures inferred from matrix dehydration and volatile trace-element loss from thermally metamorphosed CM and CI chondrites (e.g., Tonui et al., 2002, 2003, 2014; Table 3).

Diminished heating effects penetrate from the surface as far as the (spin-rate controlled) diurnal heat penetration (thermal skin) depth (Marchi et al., 2009), which is on the order of $2-3$ $\mathrm{cm}$ from the surface of an object with a rotation rate on the order of hours (e.g., Delbo and Michel, 2011) and $\sim 1.5 \mathrm{~m}$ for the annual (orbital) thermal skin depth (Mazanek et al., 2016). Material so close to the surface of a meteoroid would not survive atmospheric entry (during which typically $>90 \%$ of the incoming meteoroid is ablated or otherwise rendered unrecoverable as meteoritic material). Consequently, it is much more likely that thermally metamorphosed CM and CI chondrites were heated in their parent bodies early in solar system history than that they were heated during a recent orbital epoch with small perihelia.

It is beyond the diurnal and annual thermal skin depths that inner-Solar System small bodies and their largest rock masses (e.g., asteroid surface boulders) are expected to preserve organic compounds and other volatiles least affected by surface (regolith) processes. Regolith gardening can excavate and expose long-buried materials to the entire menu of surface smallbodies surface processes, and can bury previously exposed regolith and surface-fragment masses. The spectra of surfaces of current sample-return-mission target asteroids best match thermally anomalous $\mathrm{C}$ chondrites and experimentally heated carbonaceous chondrites (e.g., Clark et al., 2011). If regolith gardening has brought such material from the interior of pervasively metamorphosed asteroids, they represent thermally processed material, which is unlikely to preserve indigenous volatiles. However, if such surface material was heated during a past epoch of more eccentric orbit, pristine volatile-rich material is likely to occur in the interiors of boulders larger than the thermal skin depths. Either way, the properties of thermally altered carbonaceous chondrite meteorites (e.g., Tonui et al., 2002, 2003, 2014) are just as important for the planning of asteroid sample return as are the more abundant and common carbonaceous chondrites with their higher volatile abundances.

\subsection{Space weathering of carbonaceous chondrites}


Space weathering is an umbrella term covering diverse effects operating on small independent objects in space as well as in regoliths. We have already mentioned agglutinates earlier. Here, we will deal with implantation of solar noble gases in meteoroids. In the one-stage irradiation model a precursor rock starts out on a parent asteroid, buried under many meters of material that screens out cosmic rays. A collision excavates or Yarkovsky-O'Keefe-RadzievskiiPaddack (YORP) effect ejects the precursor rock as a newly liberated meteoroid which, now fully exposed to cosmic rays, orbits the Sun until it strikes the Earth, where the overlying atmosphere again shuts out almost all cosmic rays (Herzog, 2003). Thus the total exposure age, or cosmic ray exposure (CRE) age, can indicate which meteoroids derive from a common parent asteroid. However, alternative possible explanations exist for CREs so the explanation is not usually clear.

Compared to other meteorite types, carbonaceous chondrites have relatively short CRE ages (Herzog, 2003). The average CRE ages for CV, CK, and CO chondrites, are 13, 23, and 22 Myr, respectively. These averages exceed the CRE ages of the CI and CM chondrites by a factor of 10. The age distribution of CR chondrites seems to be most like that of the CV chondrites average age, $8 \mathrm{Myr}$, and range, from $1 \mathrm{Myr}$ to $25 \mathrm{Myr}$. The CRE ages of $\mathrm{CH}$ chondrites, 1 to 12 Myr, are similar to those of the $\mathrm{CR}$ and $\mathrm{CV}$ chondrites. $\mathrm{CB}$ chondrite CRE ages range from 25 to $36 \mathrm{Myr}$, unusually old for $\mathrm{C}$ chondrites (Herzog, 2003), but few CBs have been measured.

The CI and CM chondrites have unusually short CRE ages, many of them less than 1 Myr. Only lunar meteorites have such short CRE ages. Scherer and Schultz (2000) describe three possible reasons for short CRE ages: the parent asteroid orbited close to a resonance; the parent asteroid was in an Earth-crossing orbit when the meteoroid was released; and the meteoroids are so fragile that collisions destroy them if they fail to reach Earth quickly.

Nishiizumi and Caffee (2012) have reported that the CMs are unique in displaying several distinct peaks for cosmic-ray exposure (CRE) age groups, and that excavation from significant depth and exposure as small entities in space is the best explanation for the observed radionuclide data. There are either 3 or 4 CRE groups for CMs (Fig. 2.6). Zolensky et al. (2017) systematically characterized the petrography in each of the CRE age groups to determine whether the groups have significant petrographic differences with these reflecting different parent asteroid geological processing or multiple original bodies. Of particular interest were breccias consisting of multiple (up to 15) distinct CM lithologies. The degree of aqueous alteration varies with the CRE ages the CMs displaying the most aqueous alteration all have relatively short exposure ages. However, some CM with low degrees of alteration also have short exposures. This relationship is the same regardless of the number of distinctive lithologies a CM possesses. Also, there is an inverse relation between the number of distinctive lithologies in a CM and its exposure age. These relations can be explained in numerous ways. If we, for the moment, limit ourselves to models with a single CM parent body one possible explanation is that the degree of aqueous alteration and degree of lithology mixing increases with depth inside of the CM parent asteroid. This picture is consistent with the model proposed by Bland et al. (2004), where there is actual circulation of solids in the asteroid's muddy interior. In this model successive impacts would expose successively deeper regions of the CM asteroid, so that the most recently-excavated materials would come from the greatest depths, and potentially have increasing lithologic heterogeneity. However, the most likely explanation is that brecciated CMs are systematically weaker than unbrecciated CMs due to interlithological fractures, and thus have a shorted lifetime before being pulverized.

\subsection{References}


1. Abe M., Takagi Y., Kitazato K., Abe S, Hiroi T., Vilas F., Clark B.E., Abell P. A., Lederer S.M., Jarvis K.S., Nimura T., Ueda Y., and Fujiwara A. (2006) Near-Infrared Spectral Results of Asteroid Itokawa from the Hayabusa Spacecraft. Science 312, 1334-1338.

2. Abreu N. M. (2016a). Why is it so difficult to classify Renazzo-type (CR) carbonaceous chondrites? - Implications from TEM observations of matrices for the sequences of aqueous alteration. Geochimica et Cosmochimica Acta 194, 91-122.

3. Abreu N. M. (2016b). Are Phyllosilicates in CR Chondrite Matrices Generated by Hydrothermal Alteration? (Abstract). 47th Lunar and Planetary Science Conference. LPI Contribution No. 1903, p.1926.

4. Abreu N. M. (2013). A unique omphacite, amphibole, and graphite-bearing clast in Queen Alexandra Range (QUE) 99177: A metamorphosed xenolith in a pristine CR3 chondrite. Geochimica et Cosmochimica Acta, 105, 56-72.

5. Abreu N. M. (2011). Petrographic Evidence of Shock Metamorphism in CR2 Chondrite GRO 03116 (abstract). Meteoritics and Planetary Science Supplement, id.5211

6. Abreu N. M., and Brearley A. J. (2005). Carbonates in Vigarano: Terrestrial, preterrestrial, or both? Meteoritics and Planetary Science 40:609-625.

7. Abreu N. M. and Brearley A. J. (2010) Early solar system processes recorded in the matrices of two highly pristine CR3 carbonaceous chondrites, MET 00426 and QUE 99177. Geochimica et Cosmochimica Acta 74, 1146-1171.

8. Abreu N. M. and Bullock E. S. (2013). Opaque assemblages in CR2 Graves Nunataks (GRA) 06100 as indicators of shock-driven hydrothermal alteration in the CR chondrite parent body. Meteoritics \& Planetary Science 48, 2406-2429.

9. Abreu N. M. and Singletary S. (2011). Alteration History of CR2 Chondrite GRA 06100: FE-EPMA and TEM Analysis (abstract). LPI Contribution No. 1608, p.2659.

10. Airieau S., Farquhar J., Thiemens M., Leshin L., Bao H., Young E. (2005) Planetesimal sulfate and aqueous alteration in $\mathrm{CM}$ and $\mathrm{Cl}$ carbonaceous chondrites. Geochimica et Cosmochimica Acta 69, 4166-4171.

11. Akai, J., (1990a). Mineralogical evidence of heating events in Antarctic carbonaceous chondrites, Y-86720 and Y-82162. Proc. NIPR Symp. Antarct. Meteorites 3, 55-68.

12. Akai, J., (1990b). Thermal metamorphism in four Antarctic carbonaceous chondrites and its temperature scale estimated by T-T-T diagram. Antarctic Meteorites XV. Tokyo, Natl. Inst. Polar Res. 86-87.

13. Aléon J., Krot A. N. and McKeegan K. D. (2002). Calcium-aluminum-rich inclusions and amoeboid olivine aggregates from the CR carbonaceous chondrites. Meteoritics \& Planetary Science 37, 1729-1755.

14. Alexander, C. M. O.'D., R. Bowden, Fogel M. L., Howard K. T., Herd, C. D. K., and Nittler, L. R. (2012). The Provenances of Asteroids, and Their Contributions to the Volatile Inventories of the Terrestrial Planets. Science 337(6095), 721-723.

15. Alexander C. M. O'D., Fogel M., Yabuta H. and Cody G. D. (2007) The origin and evolution of chondrites recorded in the elemental and isotopic compositions of their macromolecular organic matter. Geochimica et Cosmochimica Acta 71, 4380-4403.

16. Alexander C. M. O'D., Greenwood R. C., Bowden R., Gibson J. M., Howard K. T., and Franchi I. A. (2018). A mutli-technique search for the most primitive CO chondrites. Geochimica et Cosmochimica Acta, 221, 406-420.

17. Alexander C. M. O.'D., Howard K. T., Bowden R., Fogel M. L. (2013). The classification of $\mathrm{CM}$ and $\mathrm{CR}$ chondrites using bulk $\mathrm{H}, \mathrm{C}$ and $\mathrm{N}$ abundances and isotopic compositions. Geochimica et Cosmochimica Acta 123, 244-260. 
18. Alexander C. M. O.'D., Bowden R., Fogel M. L., Howard K. T. (2015). Carbonate abundances and isotopic compositions in chondrites. Meteoritics \& Planetary Science 50, 810-833.

19. Alexander C., Hutchison R., Barber D. (1989) Origin of chondrule rims and interchondrule matrices in unequilibrated ordinary chondrites. Earth and Planetary Science Letters 95, 187-207.

20. Allen J. M., Grossman L., Davis A. M., and Hutcheon I. D. (1978) Mineralogy, textures and mode of formation of a hibonite-bearing Allende inclusion. Proceeding, 9th Lunar and Planetary Science Conference. pp. 1209-1233.

21. Amelin Y., Krot A. N., Hutcheon I. D. and Ulyanov A. A. (2002) Lead isotopic ages of chondrules and calcium-aluminum-rich inclusions. Science 297, 1678-1683.

22. Ammannito E., DeSanctis M. C., Ciarniello M., Frigeri A., Carrozzo F. G., Combe J.-Ph., Ehlmann B. L., Marchi S., McSween H. Y., Raponi A., Toplis M. J., Tosi F., Castillo-Rogez J.-C., Capaccioni F., Capria M. T., Fonte S., Giardino M., Jaumann R., Longobardo A., Joy S. P., Magni G., McCord T. B., McFadden L. A., Palomba E., Pieters C. M., Polanskey C. A., Rayman M. D., Raymond C. A., Schenk P. M., Zambon F., and Russell C. T. (2016) Distribution of phyllosilicates on the surface of Ceres. Science 358, 1006.

23. Anand M., Crawford I. A., Balat-Pichelin M., Abanades S., van Westrenen W., Péraudeau G., Jaumann R., and Seboldt W. (2012). A brief review of chemical and mineralogical resources on the Moon and likely initial in situ resource utilization (ISRU) applications. Planetary and Space Science, 74, 42-48.

24. Anders E., Grevesse N. (1989) Abundances of the elements - Meteoritic and solar. Geochim. Cosmochim. Acta 53, 197-214.

25. Bagnall P.M. (1991) The meteorite and tektite collector's handbook. A practical guide to their acquisition, preservation and display, Willmann-Bell, Richmond, VA (USA).

26. Barber D. J. (1981) Matrix phyllosilicates and associated minerals in C2M carbonaceous chondrites. Geochimica et Cosmochimica Acta 45, 945-970.

27. Barnouin-Jha O. S., Garvin J. B., Cheng A. F., Zuber M., Smith D., Neumann G., Murchie S., Robinson M., and Veverka J. (2001) Preliminary impact crater dimensions on 433 Eros from the NEAR laser range finder and imager. Lunar Planet. Sci. XXXII, Abstract \#1786. Lunar and Planetary Institute, Houston.

28. Barnouin-Jha O. S., Cheng A. F., Mukai T., Abe S., Hirata N., Nakamura R., Gaskell R. W., Saito J., and Clark B. E. (2008) Small-scale topography of 25143 Itokawa from the Hayabusa laser altimeter. Icarus 198, 108-124.

29. Barrett, P. J. (1980) The shape of rock particles, a critical review. Sedimentology 27, 291303.

30. Bart G. D. and Melosh H. J. (2010) Distributions of boulders ejected from lunar craters. Icarus, 209, 337-357.

31. Beck P., Garenne A., Quirico E., Bonal L., Montes-Hernandez G., Moynier F., and Schmitt B. (2014). Transmission infrared spectra $(2-25 \mu \mathrm{m})$ of carbonaceous chondrites ( $\mathrm{Cl}, \mathrm{CM}$, CV-CK, CR, C2 ungrouped): Mineralogy, water, and asteroidal processes. Icarus 229, 263-277.

32. Bell J. F. (1988) A probable asteroidal parent body for the CV or CO chondrites. Meteoritics 23, 256-257 (abstract).

33. Benn D.I., and Ballantyne C.K. (1993) The description and representation of particle shape. Earth Surface Processes and Landforms 18, 665-672. 
34. Bennett M. E. and McSween H. Y. Jr. (1996) Revised model calculations for the thermal histories of ordinary chondrite parent bodies. Meteoritics \& Planetary Science 31, 783792.

35. Bentley M. S., Schmied R., Mannell T., Torkar K., Jeszenszky H., Romstedt J., LevasseurRegourd A.-C., Weber I., Jesberger E. K., Ehrenfreund, P., Koeberl C., and Havnes O. (2016) Aggregate dust particles at comet 67P/Churyumov-Gerasimenko. Nature 537, 7375.

36. Berlin J., Jones R. H. and Brearley A. J. (2011) Fe-Mn systematics of type IIA chondrules in unequilibrated CO, CR, and ordinary chondrites. Meteoritics \& Planetary Science 46, 513-533.

37. Binns R. A. (1967) Structure and evolution of non-carbonaceous chondritic meteorites. Earth and Planetary Science Letters 2, 23-28.

38. Bischoff A., Barrat J.-A., Bauer K., Burkhardt C., Busemann H., Ebert S., Gonsior M., Hakenmüller J., Haloda J., Harries D., Heinlein D., Hiesinger H., Hochleitner R., Hoffmann V., Kaliwoda M., Laubenstein M., Maden C., Meier M., M. M.; Morlok A., Pack A., Ruf A., Schmitt-Kopplin P., Schönbächler M., Steele R. C. J., Spurný Pavel, and Wimmer K. (2017). The Stubenberg meteorite-An LL6 chondrite fragmental breccia recovered soon after precise prediction of the strewn field. Meteoritics \& Planetary Science 52, 1683-1703.

39. Bischoff A., Palme H., Ash R. D., Clayton R. N., Schultz L., Herpers U., Stoffler D., Grady M. M., Pillinger C. T., Spettel B., Weber H., Grund T., Endress M. and Weber D. (1993a). Paired Renazzo-type (CR) carbonaceous chondrites form the Sahara. Geochimica et Cosmochimica Acta 57, 1587-1603.

40. Bischoff A., Palme H., Schultz L., Weber D., Weber H. W., and Spettel B. (1993b). ACFER 182 and paired samples, an iron-rich carbonaceous chondrite - Similarities with ALH85085 and relationship to CR chondrites. Geochimica et Cosmochimica Acta 57, 2631-2648.

41. Bischoff, A., Rubin, A.E., Keil, K., and Stoeffler, D. (1983) Lithification opf gas-rich chondrite regolith breccias by grain boundary and localized shock melting. Earth and Planetary Science Letters 66, 1-10.

42. Bischoff A., Scott E. R. D., Metzler K., and Goodrich C. A. (2006) Nature and origins of meteoritic breccias. In Meteorites and the Early Solar System II (eds. D.S. Lauretta and H.Y. McSween, Jr.). University of Arizona Press. pp. 679-712.

43. Bizzarro M., Baker J. A., and Haack H. (2004) Mg isotope evidence for contemporaneopus formation of chondrules and refractory inclusions. Nature 431, 275-278.

44. Bland P. A., Cressey G., and Menzies, O. N (2004) Modal mineralogy of carbonaceous chondrites by X-ray diffraction and Mössbauer spectroscopy. Meteoritics \& Planetary Science 39, 3-16.

45. Bland, P.A., Jackson, M. D., Coker, R.F., Cohen B. A., Webber, J. B. W., Lee M. R., Duffy C. M., Chater R. J., Ardakani,M. G., McPhail D. S., McComb D.W., and Benedix G. K. (2009) Why aqueous alteration in asteroids was isochemical: High porosity $\neq$ high permeability. Earth Planet. Sci. Letters 287, 559-568.

46. Bland P. A., Muxworthy A. R., Collins G. S., Moore J., Davison T. M. and Ciesla F. J. (2011) Heterogeneous shock in porous chondrites: implications for Allende magnetization. Meteorit. Planet. Sci. 46, \#5275 (abstract).

47. Bland P. A., Zolensky M. E., Benedix G. K., and Sephton M. A. (2006). Weathering of Chondritic Meteorites. In Meteorites and the Early Solar System II (eds. D.S. Lauretta and H.Y. McSween, Jr.). University of Arizona Press. pp. 853- 867.

48. Blott S. J., and Pye K., 2008. Particle shape: a review and new methods of characterization and classification. Sedimentology 55, 31-63. 
49. Blum J. D., Wasserburg G. J., Hutcheon I. D., Beckett J. R., and Stolper E. M. (1988). "Domestic" origin of opaque assemblages in refractory inclusions in meteorites. Nature 331,405-409.

50. Blum J. D., Wasserburg G. J., Hutcheon I. D., Beckett J. R., and Stopler E. M. (1989). Origin of opaque assemblages in C3V meteorites-Implications for nebular and planetary processes. Geochimica et Cosmochimica Acta 53, 543-556.

51. Boggs, S. (1992) Petrology of Sedimentary Rocks ( $1^{\text {st }}$ ed.). Macmillan Publishing Co., 665 $p$.

52. Bonal L., Quirico E., Bourot-Denise M. and Montagnac G. (2006) Determination of the petrologic type of CV3 chondrites by Raman spectroscopy of included organic matter. Geochim. Cosmochim. Acta 70, 1849-1863.

53. Bonal L., Alexander C. M. O.'D., Huss G. R., Nagashima K., Quirico, E., and Beck, P. (2013). Hydrogen isotopic composition of the water in CR chondrites. Geochimica et Cosmochimica Acta 106, 111-133.

54. Bonal L., Bourot-Denise M., Quirico E., Montagnac G., and Lewin E. (2007). Organic matter and metamorphic history of $\mathrm{CO}$ chondrites. Geochimica et Cosmochimica Acta 71, 1605-1623.

55. Bonal L., Quirico E., Flandinet L., Montagnac G. (2016). Thermal history of type 3 chondrites from the Antarctic meteorite collection determined by Raman spectroscopy of their polyaromatic carbonaceous matter. Geochimica et Cosmochimica Acta 189, 312337.

56. Boss A. P. (2004) Convective cooling of protoplanetary disks and rapid giant planet formation. Astrophys. J. 610, 456-463.

57. Bourot-Denise M., Zanda B., and Hewins R. (1997) Metamorphic Transformations of Opaque Minerals in Chondrites. Workshop on Parent-Body and Nebular Modification of Chondritic Materials, LPI-technical report p. 5 and LPI abstract \#4040.pdf.

58. Bouvier A. and Wadhwa M. (2010) The age of the solar system redefined by the oldest $\mathrm{Pb}-\mathrm{Pb}$ age of a meteoritic inclusion. Nature Geoscience 3, 637-641.

59. Boynton W.V. (1975) Fractionation in the solar nebula: condensation of yttrium and the rare earth elements. Geochimica et Cosmochimica Acta 39, 569-584.

60. Brandstätter F., Bukovanská M., and Kurat G. 2003. NWA 1559: Another anomalous CK3 chondrite (abstract)? Meteoritics \& Planetary Science 38:A63.

61. Brearley A. J. (2009) Matrix olivines in the metamorphosed CK chondrite NWA 1628: Possible affinities to olivines in the matrices of oxidized CV3 chondrites and dark inclusions. 40th Lunar and Planetary Science Conference, \#1791 (abstract).

62. Brearley A. J. (2006) The action of water. In Meteorites and the Early Solar System II. (ed. D. S., Lauretta and H. Y. McSween), pp. 587-624. Arizona Univ. Press, Tucson.

63. Brearley, A. J. (2003) Nebular versus parent-body processing. In Meteorites, Comets, and Planets (ed. A. M. Davis) Treatise in Geochemistry Vol. 1 (eds. H. D. Holland and K. K. Turekian). Elsevier-Pergamon, Oxford. pp. 247-268.

64. Brearley A. J. (1993). Matrix and fine-grained rims in the unequilibrated CO3 chondrite, ALH A77307: origins and evidence for diverse, primitive nebular dust components. Geochimica et Cosmochimica Acta 57, 1521-1550.

65. Brearley, A. J. and Burger, P. V. (2009). Mechanisms of Aqueous Alteration of Type IIA Chondrule Glass in the CR Chondrite EET 92105: Insights from FIB/TEM Analysis (abstract). Meteoritics and Planetary Science Supplement, 5148.

66. Brearley A. J. and Jones R. H. (1998) Chondritic meteorites. In Planetary Materials (ed. Papike J. J.), pp. 3-01--3-398. Mineralogical Society of America, Washington, D.C. 
67. Briani G., Quirico E., Gounelle M., Paulhiac-Pison M., Montagnac G., Beck P., OrthousDaunay F.-R., Bonal L., Jacquet E., Kearsley A., and Russell, S. S. (2013). Short duration thermal metamorphism in CR chondrites. Geochimica et Cosmochimica Acta 122, 267279.

68. Brindley G. W. and Brown, G. (1984). Crystal structures of clay minerals and their X ray identification. Mineralogical Society of London. Spottiswoode Ballantyne Ltd. UK. pp. 495.

69. Brindley, G. W. and Hayashi R. (1965). Mechanism of formation of forsterite and enstatite from serpentine. Miner. Mag. 35, 189-195.

70. Britt D. T., Macke R. J., Kiefer W. S., Irving A. J., Hupé G., and Consolmagno G. J. (2012). The density, porosity, and magnetic susceptibility of two recent meteorite falls: Tissint and Sutter's Mill (abstract). Meteoritics and Planetary Science Supplement, id.5350

71. Brown P., Hildebrand A., Zolensky M., Grady M., Clayton R., Mayeda T., Tagliaferri E., Spalding R., MacRae N., Hoffman E., Mittlefehldt D., Wacker J., Bird J., Campbell M., Carpenter R., Gingerich H., Glatiotia M., Grainer E., Mazur M., McCausland P., Plotkin H., Rubak-Mazur T. (2000) The fall, recovery, orbit, and composition of the Tagish Lake meteorite: a new type of carbonaceous chondrite, Science 290, 320-325.

72. Browning, L. B., McSween, H. Y., Jr., and Zolensky, M. E. (1996) Correlated alteration effects in CM carbonaceous chondrites. Geochimica et Cosmochimica Acta 60, 26212633.

73. Brownlee D., Tsou P., Aléon J., Alexander C. M. O’D., Araki T., Bajt S., Baratta G. A., Bastien R., Bland P., Bleuet P., Borg J., Bradley J. P., Brearley A., Brenker F., Brennan S., Bridges J. C., Browning N. D., Brucato J. R., Bullock E., Burchell M. J., Busemann H., Butterworth A., Chaussidon M., Cheuvront A., Chi M., Cintala M. J., Clark B. C., Clemett S. J., Cody G., Colangeli L., Cooper G., Cordier P., Daghlian C., Dai Z., D'Hendecourt L., Djouadi Z., Dominguez G., Duxbury T., Dworkin J. P., Ebel D. S., Economou T. E., Fakra S., Fairey S. A. J., Fallon S., Ferinni G., Ferroir T., Fleckenstein H., Floss C., Flynn G., Franchi I. A., Fries M., Gainsforth Z., Gallien J.-P., Genge M., Gilles M. K., Gillet P., Gilmour J., Glavin D. P., Gounelle M., Grady M. M., Graham G. A., Grant P. G., Green S. F., Grossemy F., Grossman L., Grossman J. N., Guan Y., Hagiya K., Harvey R., Heck P., Herzog G. F., Hoppe P., Hörz F., Huth J., Hutcheon I. D., Ignatyev K., Ishii H., Ito M., Jacob D., Jacobsen C., Jacobson S., Jones S., Joswiak D., Jurewicz A., Kearsley A. T., Keller L. P., Khodja H., Kilcoyne A. L. D., Kissel J., Krot A., Langenhorst F., Lanzirotti A., Le L., Leshin L. A., Leitner J., Lemelle L., Leroux H., Liu M.-C., Luening K., Lyon I., MacPherson G., Marcus M. A., Marhas K., Marty B., Matrajt G., McKeegan K., Meibom A., Mennella V., Messenger K., Messenger S., Mikouchi T., Mostefai S., Nakamura T., Nakano T., Newville M., Nittler L. R., Ohnishi I., Ohsumi K., Okudaira K., Papanastassiou D. A., Palma R., Palumbo M. E., Pepin R. O., Perkins D., Perronnet M., Pianetta P., Rao W., Rietmeijer F. J. M., Robert F., Rost D., Rotundi A., Ryan R., Sandford S. A., Schwandt C. S., See T. H., Schlutter D., Sheffield-Parker J., Simionovici A., Simon S., Sitnitsky I., Snead C. J., Spencer M. K., Stademann F. J., Steele A., Stephan T., Stroud R., Susini J., Sutton S. R., Suzuki Y., Taheri M., Taylor S., Teslich N., Tomeoka K., Tomioka N., Toppani A., Trigo-Rodríguez J. M., Troadec D., Tsuchiyama A., Tuzzolino A. J., Tyliszczak T., Uesugi K., Velbel M., Vellenga J., Vicenzi E., Vincze L., Warren J., Weber I., Weisberg M., Westphal A. J., Wirick S., Wooden D., Wopenka B., Wozniakiewicz P., Wright I., Yabuta H., Yano H., Young E. D., Zare R. N., Zega T., Ziegler K., Zimmerman L., Zinner E. and Zolensky M. (2006) Comet 81P/Wild 2 Under a Microscope. Science 314, 17111716. 
74. Buchanan P.C., Zolensky M.E. and Reid A.M. (1993) Carbonaceous chondrite clasts in HED polymict breccias. Meteoritics 28, 659-682.

75. Buchanan P., Zolensky M., Weisberg M., Hagiya K., Mikouchi T., Takenouchi A., Hasegawa H., Ono H., Higashi K., Ohsumi K. (2017) Oriented mineral transformation in a dark inclusion from the Leoville meteorite. 48th Lunar and Planetary Science Conference. Abstract.

76. Budde G., Kruijer T. S., and Kleine T. (2018). Hf-W chronology of CR chondrites: Implications for the timescales of chondrule formation and the distribution of ${ }^{26} \mathrm{Al}$ in the solar nebula. Geochimica et Cosmochimica Acta, 222, 284-304.

77. Bukovanská M., Brandstätter F., and Kurat G. 2003. NWA 1560 (CK4/5) and NWA 1563 (CK5) - A comparison with $\mathrm{HaH} 280$ (CK4) chondrite (abstract). Meteoritics \& Planetary Science 38, A84.

78. Bullock E. S., Gounelle M., Lauretta D. S., Grady M. M., Russell S. (2005) Mineralogy and texture of Fe-Ni sulfides in Cl1 chondrites: clues to the extent of aqueous alteration on the Cl1 parent body. Geochimica et Cosmochimica Acta 69, 2687-2700.

79. Bunch, T. E. (1975). Petrography and petrology of basaltic achondrite polymict breccias /Howardites. $6^{\text {th }}$ Lunar Science Conference Proceedings, 1, p. 469-492.

80. Bunch, T. E. and Chang, S. (1980) Carbonaceous chondrites - II. Carbonaceous chondrite phyllosilicates and light element geochemistry as indicators of parent body processes and surface conditions. Geochimica et Cosmochimica Acta 44, 1543-1577.

81. Burger, P. V. and Brearley, A. J. (2005). Localized Chemical Redistribution During Aqueous Alteration in CR2 Carbonaceous Chondrites EET 87770 and EET 92105 (abstract). 36th Annual Lunar and Planetary Science Conference, abstract no.2288.

82. Burger, P. V. and Brearley, A. J. (2004). Chondrule Glass Alteration in Type IIA Chondrules in the CR2 Chondrites EET 87770 and EET 92105: Insights into Elemental Exchange Between Chondrules and Matrices. 35th Lunar and Planetary Science Conference, abstract no.1966.

83. Burton A., Mclain H., Glavin D., Elsila J., Davidson J., Miller K., Andronikov A., Lauretta D., Dworkin J. (2015) Amino acid analyses of R and CK chondrites. Meteoritics \& Planetary Science 50, 470-482.

84. Busemann H., Alexander C. M. O'D., and Nittler L. R. (2007) Characterization of insoluble organic matter in primitive meteorites by microRaman spectroscopy. Meteoritics and Planetary Science 42, 1387-1416.

85. Campbell A. J., Humayun M., and Weisberg M. K. (2002) Siderophile element constraints on the formation of metal in the metal-rich chondrites Bencubbin, Weatherford, and Gujba. Geochimica et Cosmochimica Acta 66, 647-660.

86. Campbell A. J., Humayun M., and Zanda B. (2002). Partial condensation of volatile elements in Renazzo chondrules. Geochimica et Cosmochimica Acta 66, A117.

87. Campbell A. J., Zanda B., Perron C., Meibom A., and Petaev M. I. (2005). Origin and thermal history of Fe-Ni metal in primitive chondrites. In Chondrites and the protoplanetary disk, edited by Krot A. N., Scott E. R. D., and Reipurth B. Astronomical Society of the Pacific Conference Series, vol. 341. pp. 407-431.

88. Cantando, E. D., Dukes, C. A., Loeffler, M. J., and Baragiola, R. A. (2008) Aqueous depletion of $\mathrm{Mg}$ from olivine surfaces enhanced by ion irradiation. Journal of Geophysical Research 113, E09011, doi:10.1029/2008JE003119.

89. Carporzen L., Weiss B. P., Elkins-Tanton L. T., Shuster D. L., Ebel D. and Gattacceca J. (2011) Magnetic evidence for a partially differentiated carbonaceous chondrite parent body. Proc. Natl. Acad. Sci. USA 108, 6386-6389. 
90. Casanova I. and Simon S. B. (1994) Opaque minerals in CAls, and classification of the Axtell (CV3) chondrite (abstract). Meteoritics 29, 454-455.

91. Cato M., Fagan A. (2017) An overview of type ii chondrules in the CM chondrite Jbilet Winselwan. Lunar and Planetary Science XLVIII. abstract 2676.

92. Chapman C. R. (2002) Cratering on asteroids from Galileo and NEAR Shoemaker. In Asteroids III (W. F. Bottke Jr. et al., eds.), pp. 315-330. Univ. of Arizona, Tucson.

93. Chaumard N. and Devouard B. (2015) Zoned plagioclases in the matrices of CK carbonaceous chondrites. 46th Lunar and Planetary Science Conference, \#1924 (abstract).

94. Chaumard N. and Devouard B. (2016) Chondrules in CK carbonaceous chondrites and thermal history of the CV-CK parent body. Meteoritics and Planetary Science 51, 547573.

95. Chaumard N., Devouard B., Zanda B., and Ferrière L. (2009a) The link betwen CV and CK chondrites based on parent body processes. Meteoritics and Planetary Science Supplement 44, \#5206 (abstract).

96. Chaumard, N., Devouard, B., Zanda, B. and Ferrière, L. (2009b). The link between CV and CK carbonaceous chondrites based on parent body processes. Meteorit. Planet. Sci. 44, \#5206 (abstract).

97. Chaumard N., Devouard B., Zanda B., and Devidal J.-L. (2011) Metamorphosed calciumaluminum-rich inclusions in the Tanezrouft 057 CK4 carbonaceous chondrite. 42nd Lunar and Planetary Science Conference, \#1608 (abstract).

98. Chaumard N., Devouard B., Delbo M., Provost A. and Zanda B. (2012) Radiative heating of carbonaceous near-Earth objects as a cause of thermal metamorphism for CK chondrites. Icarus 220, 65-73.

99. Chaumard N., Devouard B., Bouvier A., and Wadhwa M. (2014) Metamorphosed calciumaluminum-rich inclusions in $\mathrm{CK}$ carbonaceous chondrite. Meteoritics and Planetary Science 49, 419-452.

100. Cheng A. F. (2002) Near Earth asteroid rendezvous: Mission summary. In Asteroids III (W. F. Bottke Jr. et al., eds.), pp. 351-366. Univ. of Arizona, Tucson.

101. Cheng A. F., Barnouin-Jha O. S., Hirata N., et al., (2007) Fundamentally distinct outcomes of asteroid collisional evolution: Itokawa and Eros. Journal of Geophysical Research 34, DOI: 10.1029/2007GL029559.

102. Cheng A.F., Izenberg N., Chapman C.R. and Zuber M.T. (2002) Ponded deposits on asteroid 433 Eros. Meteoritics and Planetary Science 37, 1095-1105.Chizmadia L. J. and Brearley A. J. (2008) Mineralogy, aqueous alteration, and primitive textural characteristics of fine-grained rims in the Y-791198 CMS carbonaceous chondrite: TEM observations and comparison to ALHA81002. Geochimica et Cosmochimica Acta 72, 602-625.

103. Chizmadia L. J. and Brearley A. J. (2008) Mineralogy, aqueous alteration, and primitive textural characteristics of fine-grained rims in the Y-791198 CMS carbonaceous chondrite: TEM observations and comparison to ALHA81002. Geochimica et Cosmochimica Acta 72, 602-625.

104. Chizmadia L. J., Rubin A. E., and Wasson J. T. (2002). Mineralogy and petrology of amoeboid olivine inclusions in $\mathrm{CO} 3$ chondrites; Relationships to parent body aqueous alteration. Meteoritics \& Planetary Science 37:1781-1796.

105. Choi B.-G. and Wasson J. T. (2003) Microscale oxygen isotopic exchange and formation of magnetite in the Ningqiang anomalous carbonaceous chondrite. Geochimica et Cosmochimica Acta 67, 4655-4660. 
106. Choi B.-G., McKeegan K. D., Leshin L. A., and Wasson J. T. (1997) Origin of the magnetite in oxidized CV chondrites: In situ measurement of oxygen isotopic compositions of Allende magnetite and olivine. Earth and Planetary Science Letters 146, 337-349.

107. Choi B.-G., McKeegan K. D., Krot A. N., and Wasson J. T. (1998) Extreme oxygen isotopic compositions in magnetite from unequilibrated ordinary chondrites. Nature 392, 577-579.

108. Choi B.-G., Krot A. N., and Wasson J. T. (2000) Oxygen-isotopes in magnetite and fayalite in CV chondrites Kaba and Mokoia. Meteoritics \& Planetary Science 35, 12391248.

109. Ciesla F. J. (2009) Two-dimensional transport of solids in viscous protoplanetary disks. icarus 200, 655-671.

110. Ciesla F. J. (2010) The distributions and ages of refractory objects in the solar nebula. Icrus 208, 455-467.

111. Ciesla F. and Lauretta D. (2005) Radial migration and dehydration of phyllosilicates in the solar nebula. Earth Planet. Sci. Lett. 231, 1-8.

112. Clark B. E., Binzel R. P., Howell E. S., Cloutis E. A., Ockert-Bell, M., Christensen P., Barucci M. A., DeMeo F., Lauretta D. S., Connolly Jr., H., Soderberg A., Hergenrother C.., Lim L., Emery J. and Mueller M. (2011) Asteroid (101955) 1999 RQ36: Spectroscopy from 0.4 to $2.4 \mu \mathrm{m}$ and meteorite analogs. Icarus 216, 462-475.

113. Clayton R. N. (1993) Oxygen isotopes in meteorites. Annual Reviews in Earth and Planetary Science 21, 115-149.

114. Clayton R. N. and Mayeda T. K. (1999) Oxygen isotope studies of carbonaceous chondrites. Geochimica et Cosmochimica Acta 63, 2089-2104.

115. Clayton R. N., Onuma N., Grossman L. and Mayeda T. K. (1977) Distribution of the presolar component in Allende and other carbonaceous chondrites. Earth Planet. Sci. Lett. 34, 209-224.

116. Clayton R. N., MacPherson G. J., Hutcheon I. D., Davis A. M., Grossman L., Mayeda T. K., Molini-Velsko C., Allen J. M. and El Goresy A. (1984) Two forsterite-bearing FUN inclusions in the Allende meteorite. Geochimica et Cosmochimica Acta 48, 535-548.

117. Cohen R. E., Kornacki A. S. and Wood J. A. (1983) Mineralogy and petrology of chondrules and inclusions in the Mokoia CV3 chondrite. Geochimica et Cosmochimica Acta 47, 1739-1757.

118. Connelly H. C. Jr., Bizzarro M., Krot A., Nordlunds A., Wielandt D., Ivanova M. (2012) The absolute chronology and thermal processing of solids in the solar protoplanetary disk. Science 338, 651-655.

119. Connolly H. C. Jr., Hewins R. H., Ash R. D., Zanda B., Lofgren G. E., and BourotDenise J. (1994). Carbon and the formation of reduced chondrules. Nature 371,136-139.

120. Connolly, Jr., H. C., Huss G. R. and Wassenburg G. J. (2001) On the formation of $\mathrm{Fe}-\mathrm{Ni}$ metal in Renazzo-like carbonaceous chondrites. Geochimica et Cosmochimica Acta 65, 4567-4588.

121. Connolly, Jr., H. C. and Huss G. R. (2010) Compositional evolution of the protoplanetary disk: Oxygen isotopes of type-II chondrules from CR2 chondrites. Geochimica et Cosmochimica Acta 74, 2473-2483.

122. Connolly, Jr, H. C. and Jones R. H. (2016) Chondrules: canonical and noncanonical views. Journal of Geophysical Research Planets 121, 1885- 1899.

123. Connolly H. C., Jr., Lauretta D. S., Walsh K. J., Tachibana S., and Bottke W. F., Jr. (2015) Toward understanding the dynamical evolution of asteroid 25143 Itokawa: constraints from sample analysis. Earth, Planets and Space 67, article \#12. 
124. Consolmagno G., Schaefer M., Bradley E. Schaefer B., Daniel T. Britt D., Robert J. Macke R., Michael C. Nolan M., Ellen S. Howell E. (2013) The measurement of meteorite heat capacity at low temperatures using liquid nitrogen vaporization. P\&SS 87, 146-156.

125. Consolmagno G. J. and Britt D. T. (1998). The density and porosity of meteorites from the Vatican collection. Meteoritics \& Planetary Science 33:1231-1241.

126. Consolmagno G. J., Britt D. T., and Stoll C. P. (1998). The porosities of ordinary chondrites: Models and interpretation. Meteoritics \& Planetary Science 33:1221-1229.

127. Consolmagno G. J., Macke R. J., Rochette P., Britt D. T., and Gattacceca J. (2006). Density, magnetic susceptibility, and the characterization of ordinary chondrite falls and showers. Meteoritics \& Planetary Science 41:331-342.

128. Consolmagno G. J., Britt D. T., and Macke R. J. (2008). The significance of meteorite density and porosity. Chemie der Erde-Geochemistry 68:1-29.

129. Corrigan C. C., Zolensky M. E., Dahl J., Long M., Weir J., Sapp C. (1997) The porosity and permeability of chondritic meteorites and interplanetary dust particles. Meteoritics and Planetary Science 32, 509-515.

130. Cuzzi J. N. and Hogan R. C. (2003) Blowing in the wind I. Velocities of chondrulesized particles in a turbulent protoplanetary nebula. Icarus 164, 127-138.

131. Cuzzi J. N. and Weidenschilling S. J. (2006) Particle-gas dynamics and primary accretion. In Meteorites and the Early Solar System II, (ed. Lauretta D. S. and McSween H. Y.), pp. 353-381. University of Arizona Press, Tucson.

132. Cuzzi J. N., Davis S. S. and Dobrovolskis A. R. (2003) Blowing in the wind. II. Creation and redistribution of refractory inclusions in a turbulent protoplanetary nebula. Icarus 166, 385-402.

133. Cuzzi J. N., Ciesla F. J., Petaev M. I., Krot A. N., Scott E. R. D. and Weidenschilling S. J. (2005) Nebular evolution of thermally processed solids: Reconciling models and meteorites. In Chondruoles and the Protoplanetary Disk (ed. Krot A. N., Scott E. R. D. and Reipurth B.) pp. 732-773. Astronomical Society of the Pacific, San Francisco.

134. Davis A. M. and Grossman L. (1979) Condensation and fractionation of rare earths in the solar nebula. Geochim. Cosmochim. Acta 43, 1611-1632.

135. Davidson J., Lauretta D. S., and Schrader D. L. (2011) Compositional variations in opaque phases within the CV and CK carbonaceous chondrites. 42nd Lunar and Planetary Science Conference, \#1886 (abstract).

136. Davidson J., Busemann H., Nittler L. R., Alexander C. M. O.'D., Orthous-Daunay F.-R., Franchi I. A., and Hoppe P. (2014a). Abundances of presolar silicon carbide grains in primitive meteorites determined by NanoSIMS. Geochimica et Cosmochimica Acta, 139, 248-266.

137. Davidson J., Nittler L. R., Alexander C. M. O'D., and Stroud, R. M. (2014b) Petrography of Very Primitive CO3 Chondrites: Dominion Range 08006, Miller Range 07687, and Four Others (abstract). LPI Contribution No. 1777, p.1384.

138. Davidson J., Krot A. N., Nagashima K., Hellebrand E., and Lauretta D. S. (2014c). Oxygen isotope and chemical compositions of magnetite and olivine in the anomalous CK3 Watson 002 and ungrouped Asuka-881595 carbonaceous chondrites: Effects of parent body metamorphism. Meteoritics \& Planetary Science 35, 1456-1474.

139. Delbo M. and Michel P. (2011) Temperature history and dynamical evolution of (101955) $1999 \mathrm{RQ} 36$ : A potential target for sample return from an primitive asteroid. Astrophysical Journal Letters 728, L42-L46. 
140. Delbo, M., Libourel, G., Wilkerson, J., Murdoch, N., Michael, P., Ramesh, K.T., Ganino, C., Verati, C., and Marchi, S. (2014) Thermal fatigue as the origin of regolith on small asteroids. Nature 508, 233-236.

141. de Leuw S., Rubin A., Wasson J. (2010) Carbonates in CM chondrites: Complex formational histories and comparison to carbonates in $\mathrm{Cl}$ chondrites. Meteoritics \& Planetary Science 45, 513-530.

142. Devouard B., Ferrière L., Zanda-Hewins B., and Messaoudi M. (2006) Mineralogy and petrology of TNZ 057 (C4) and comparison to the CV and CK groups. Meteoritics and Planetary Science 41, A203 (abstract).

143. Divine N. (1993) Five polulations of interplanetary meteoroids. Journal of Geophysical Research 98, 17029-17048.

144. Dobrică, E., and Ogliore, R.C. (2016) Adhering grains and surface features on two Itokawa particles. Earth, Planets and Space 68, article \#21.

145. Dodd R. T. (1981) Meteorites: A Petrologic-Chemical Synthesis. Cambridge University Press, $368 \mathrm{pp}$.

146. Dombard A. J., Barnouin O. S., Prockter L. M., et al., (2010) Boulders and ponds on the asteroid 433 Eros. Icarus 210, 713-721.

147. DuFresne E. R. and Anders E. (1962) On the chemical evolution of the carbonaceous chondrites. Geochimica et Cosmochimica Acta 26, 1085-1114.

148. Dukes C. A., Baragiola R. A., and McFadden L. A. (1999) Surface modification of olivine by $\mathrm{H}+$ and $\mathrm{He}+$ bombardment. J. Geophys. Res. 104:E1865-1872.

149. Dunn T. S. (2011) Nickel abundance of olivine and magnetite in CV and CK chondrites: Evidence for a continuous metamorphic sequence? 42nd Lunar and Planetary Science Conference, \#2043 (abstract).

150. Dunn T. L. (2014) Nickel in type II chondrules olivine as an indicator of petrologic subtype in CK chondrites. 45th Lunar and Planetary Science Conference, \#1038 (abstract).

151. Dunn T. S., Gross J., Ivanova M. A., Runyon S. E., and Bruck A. M. (2016a) Magnetite in the unequilibrated CK chondrites: Implications for metamorphism and new insights into the relationship between the CV and CK chondrites. Meteoritics and Planetary Science 1-20.

152. Dunn T. S., Gross J., and Ivanova M. A. (2016b) Homogeneity of matrix and chondrule olivine in the unequilibrated CK chondrites. Meteoritics and Planetary Science 51, \#6429 (abstract).

153. Dunn T. L. and Gross J. (2017) Reclassification of Hart and Northwest Africa 6047: Criteria for distinguishing between CV and CK3 chondrites. Meteoritics and Planetary Science 52, 2412-2423.

154. Durda D. D., Bagatin A. C., Aleman R. A., Flynn G. J., Strait M. M., Clayton A. N., and Patmore E. B. (2015) The shapes of fragments from catastrophic disruption events: Effects of target shape and impact speed. Planetary and Space Science 107, 77-83.

155. Ebel D. S., Brunner C., Konrad K., Leftwich K., Erb I., Lu M., Rodriguez H., Crapster-Pregont E. J., Friedrich J. M., and Weisberg M. K. (2016). Abundance, major element composition and size of components and matrix in CV, CO and Acfer 094 chondrites. Geochimica et Cosmochimica Acta, 172, 322-356.

156. Ebel D. S., Weisberg M. K., Hertz J. and Campbell A. J. (2008) Shape, metal abundance, chemistry, and origin of chondrules in the Renazzo (CR) chondrite. Meteoritics \& Planetary Science 43, 1725-1740. 
157. Ehlers K., El Goresy A. (1988) Normal and reverse zoning in niningerite: A novel key parameter to the thermal histories of $\mathrm{EH}$-chondrite. Geochimica et Cosmochimica Acta 52, 877-887.

158. Elkins-Tanton L. T., Weiss B. P. and Zuber M. T. (2011) Chondrites as samples of differentiated planetesimals. Earth Planet. Sci. Lett. 305, 1-10.

159. Endress M., Bischoff A. (1996) Carbonates in Cl chondrites: clues to parent body evolution Geochimica et Cosmochimica Acta 60, 489-507.

160. Endress M., Keil K., Bischoff A., Spettel B., Clayton R. N., and Mayeda T. K. (1994). Origin of dark clasts in the ACFER 059/EI Djouf 001 CR2 chondrite. Meteoritics 29, 26-40.

161. Eugster O., Herzog G. F., Marti K., and Caffee M. W. (2006) Irradiation records, cosmic-ray exposure ages, and transfer times of meteorites, in Lauretta, D.S., and McSween, H.Y., Jr., eds., Meteorites and the Early Solar System II: Tucson, The University of Arizona Press, p. 829-851.

162. Fagan T. J., Guan Y., MacPherson G. J., and Huss G. R. (2005) Al-Mg isotopic evidence for separate nebular and parent-body alteration events in two Allende CAls. 36th Lunar and Planetary Science Conference, \#1820 (abstract)

163. Fagan T. J., Guan Y., and MacPherson G. J. (2006) Al-Mg isotopic constraints on alteration of Allende Ca-Al-rich inclusions. 37th Lunar and Planetary Science Conference, \#1213 (abstract).

164. Fagan T., Guan Y., and MacPherson G. J. (2007) Al-Mg isotopic evidence for episodic alteration of $\mathrm{Ca}$-Al-rich inclusions from Allende. Meteoritics \& Planetary Science 42, 1221-1240.

165. Fagan T., Krot A., Keil K. (2000) Calcium-aluminum-rich inclusions in enstatite chondrites (!): Mineralogy and textures. Meteorit. Planet. Sci. 35, 771-781.

166. Fagan T., McKeegan K., Krot A., Keil K. (2001) Calcium-aluminum-rich inclusions in enstatite chondrites (II): Oxygen isotopes. Meteorit. Planet. Sci. 36, 223-230.

167. Fahey A., Goswami J. N., McKeegan K. D., and Zinner E. (1987) ${ }^{26} \mathrm{Al},{ }^{244} \mathrm{Pu},{ }^{50} \mathrm{Ti}$, REE, and trace element abundances in hibonite grains from CM and CV meteorites. Geochim. Cosmochim. Acta 51, 329-350.

168. Fahey A. J., Zinner E., Kurat K., and Kracher A. (1994). Hibonite-hercynite inclusion $\mathrm{HH}-1$ from the Lancé (CO3) meteorite: the history of an ultrarefractory CAl. Geochimica et Cosmochimica Acta 58, 4779-4793.

169. Farinella P., Froeschlé C., Froeschlé C., Gonczi G., Hahn G., Morbidelli A., and Valsecchi G. B. (1994) Asteroids falling into the Sun. Nature 371, 314-317.

170. Fleet M. E., Herzberg C. T., Bancroft G. M., and Aldridge L. P. (1978) Omphacite studies; I, The P2/n- > C2/c transformation. Americal Mineralogist 63, 1100-1106.

171. Floss C., Le Guillou C., and Brearley A. (2014). Coordinated NanoSIMS and FIBTEM analyses of organic matter and associated matrix materials in CR3 chondrites. Geochimica et Cosmochimica Acta, 139, 1-25.

172. Floss C. and Stadermann F. (2009). Auger Nanoprobe analysis of presolar ferromagnesian silicate grains from primitive CR chondrites QUE 99177 and MET 00426. Geochimica et Cosmochimica Acta, 73, 2415-2440.

173. Flynn G. (2015) The physical properties of meteorites and interplanetary dust particles: implications for the properties of stone asteroids. Spacecraft Reconnaissance of Asteroid and Comet Interiors. 6005. 
174. Flynn G. J., Consolmagno G. J., Brown P., Macke R. J. (2017). Physical properties of the stone meteorites: Implications for the properties of their parent bodies. Chemie der Erde.

175. Franchi I. A. (2008) Oxygen isotopes in asteroidal materials. In Reviews in Mineralogy and Geochemistry Volume 68: Oxygen in the solar system. (eds. MacPherson G. J., Mittlefehldt D. W., Jones J. H., and Simon S. B.), pp. 345-397.

176. Frank D., Huss G., Nagashima K., Zolensky M., Le L. (2017) Oxygen, magnesium, and aluminum isotopes in the Ivuna CAI: re-examining high-temperature fractionations in $\mathrm{Cl}$ chondrites. 80th Annual Meeting of the Meteoritical Society, Abstracts.

177. Fredriksson, K., Kerridge, J.F. (1988) Carbonates and sulfates in Cl chondrites: formation by aqueous activity of the parent body. Meteoritics 23, 35-44.

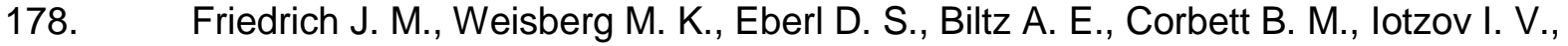
Khan W. S. and Wolman M. D. (2015) Chondrule size and related physical properties: A compilation and evaluation of current data across all meteorite groups. Chem. Erde 75, 419-443.

179. Friend P., Hezel D., Barrat J.-A., Zipfel J., Palme H. (2016) The chemical composition of matrix, chondrules and bulk of the CM chondrite Jbilet Winselwan. 47th Lunar and Planetary Science Conference, abstract 1893.

180. Fujiwara A., Kamimoto G., Tsukamoto A., (1978) Expected shape distribution of asteroids obtained from laboratory impact experiments. Nature 272, 602-603.

181. Fujiwara A., Kawaguchi J., Yeomans D. K., et al. (2006) The rubble pile asteroid Itokawa as observed by Hayabusa. Science 312,1330-1334.

182. Gardner-Vandy K. G., Lauretta D. S., Greenwood R. C., McCoy T. J., Killgore M., Franchi I. A. (2012) The Tafassasset primitive achondrite: Insights into initial stages of planetary differentiation. Geochimica et Cosmochimica Acta, 85, 142-159.

183. Garenne A., Beck P., Montes-Hernandez G., Chiriac R., Toche F., Quirico E., Bonal, L., and Schmitt B. (2014). The abundance and stability of "water" in type 1 and 2 carbonaceous chondrites ( $\mathrm{Cl}, \mathrm{CM}$ and CR). Geochimica et Cosmochimica Acta 137, 93112.

184. Garvie L., Knauth P., Morris M. (2017) Sedimentary laminations in the Isheyevo $(\mathrm{CH} / \mathrm{CBb})$ carbonaceous chondrite formed by gentle impact-plume sweep-up. Icarus 292, 36-42.

185. Gattacceca J., Krzesinska A., Marrocchi Y., Meier M., Bourot-Denise M., Lennsen R. (2017) Young asteroid mixing revealed in ordinary chondrites: The case of NWA 5764, a polymict LL breccia with L clasts. Meteoritics \& Planetary Science 52, 2289-2304.

186. Geiger T. and Bischoff A. (1990) exsolution of spinel and ilmenite in magnetites from type 4-5 carbonaceous chondrites - indications for metamorphic processes. 21st Lunar and Planetary Science Conference, 409-410 (abstract).

187. Geiger T. and Bischoff A. (1991) The CK chondrites-Conditions of parent body metamorphism. Meteoritics, 26, 337 (abstract).

188. Geiger T. and Bischoff A. (1995) Formation of opaque minerals in CK chondrites. Planet. Space Sci. 43, 485-498.

189. Geiger T., Metzler K., Bischoff A., Köhler A. V., and Arndt J. (1989) Annealing experiments on Allende (CV): textural and mineralogical modifications. 20th Lunar and Planetary Science Conference, 337-338 (abstract).

190. Geiger T., Spettel B., Clayton R. N., Mayeda T. K., and Bischoff A. 1993. Watson 002 - The first CK/Type 3 chondrite (abstract). Meteoritics 28, 352. 
191. Ghosh A. and McSween H. Y. (1998) A thermal model for the differentiation of asteroid 4 Vesta, based on radiogenic heating. Icarus 134, 187-206.

192. Ghosh A., Weidenschilling S. J., McSween H. Y., and Rubin A. E. (2006) Asteroidal heating and thermal stratification of the asteroidal belt. In Meteorites and the Early Solar System II (eds. Lauretta D. S. and McSween Jr. H. Y.). University of Arizona Press, Tucson, pp. 555-566.

193. Gillis-Davis, J. J., Gasda, P.J., Bradley, J. P., Ishii, H. A., and Bussey, D. B. J. (2015) Laser space weathering of Allende (CV3) and Murchison (CMs) carbonaceous chondrites. LPSC XLVI, abst. \# 1607.

194. Gilmour I. (2005) Structural and isotopic analysis of organic matter in carbonaceous chondrites. In Meteorites, Comets and Planets (ed. Davis A. M.), pp. 269290. Treatise on Geochemistry, Elsevier, Amsterdam.

195. Glavin D. P., Alexander C. M. O’D., Aponte J. C., Dworkin J. P., Elsila, J. E. and Yabuta H. (2018). The Origin and Evolution of Organic Matter in Carbonaceous Chondrites and Links to Their Parent Bodies.. In Primitive Meteorites and Asteroids (ed. N. M. Abreu). Elsevier.

196. Gooding J. L. and Keil K. (1981) Relative abundances of chondrule primary textural types in ordinary chondrites and their bearing on conditions of chondrule formation. Meteoritics 16,17-43.

197. Goodrich C., Fioretti A., Zolensky M., Fries M., Shaddad M., Kohl I., Young E., Jenniskens P. (2017) A breccia of ureilitic and C2 carbonaceous chondrite materials from Almahata Sitta: implications for the regolith of ureilitic asteroids. 80th Annual Meeting of the Meteoritical Society, Abstracts.

198. Gounelle M. and Zolensky M. (2001) A terrestrial origin for sulfate veins in Cl1 chondrites. Meteoritics and Planetary Science 36, 1321-1329.

199. Gounelle M., Krot A. N., Nagashima K. and Kearsley A. (2009). Extreme ${ }^{16} \mathrm{O}$ enrichment in calcium-aluminum-rich inclusions from the Isheyevo $(\mathrm{CH} / \mathrm{CB})$ chondrite. The Astrophysical Journal 698, L18-L22.

200. Gounelle M., Shu F. H., Shang H., Glassgold A. E., Rehm K. E. and Lee T. (2001) Extinct radioactivities and protosolar cosmic rays: Self-shielding and light elements. Astrophys. J. 548, 1051-1070.

201. Gradie J. and Tedesco E. (1982) Compositional structure of the asteroid belt. Science 216, 1405-1407.

202. Grady M. M. (2000) Catalog of Meteorites, Fifth ed. Cambridge University Press, pp. 689.

203. Grady M., Pratesi G., Cecchi V. (2014) Atlas of Meteorites. Cambridge University Press, 373pp.

204. Greenwood R. C. and Franchi I. A. (2004). Alteration and metamorphism of CO3 chondrites: evidence from oxygen and carbon isotopes. Meteoritics \& Planetary Science, 39, 1823-1838.

205. Greenwood R. C., Franchi I. A., Chaumard N., Devouard B., and Burbine T. H. (2010b) Is the Eos family derived from the breakup of a stratified CV-CK parent body? Meteoritics and Planetary Science 45, \#5430 (abstract).

206. Greenwood R. C., Franchi I. A., Kearsley A. T. and Alard O. (2010a) The relationship between CK and CV chondrites. Geochim. Cosmochim. Acta 74, 1684-1705.

207. Greenwood R. C., Franchi I. A., Kearsley A. T., and Alard O. (2004) The relationship between $\mathrm{CK}$ and $\mathrm{CV}$ chondrites: A single parent body source? 35th Lunar and Planetary Science Conference, \#1664 (abstract). 
208. Greenwood R., Franchi I., Zolensky M., Buchanan P. (2016) The origin and significance of the CCAM line: evidence from chondrules and dark inclusions in Allende (CV3). 47th Lunar and Planetary Science Conference. Abstract.

209. Greenwood R. C., Kearsley A. T., and Franchi I. A. (2003) Are CK chondrites really a distinct group or just equilibrated CVs? Meteoritics and Planetary Science 38, A96 (abstract).

210. Greenwood J. P., McKeegan K. D., and Wasson J. T. (2000b) A Karoonda conundrum: Primordial oxygen in magnetite, olivine, and iron-rich spinel in a metamorphism calcium-aluminum-rich inclusions. Meteoritics and Planetary Science Supplement 35, A63 (abstract).

211. Greenwood J., Rubin A. E., Kallemeyn G., and Wasson J. T. (2000c) Oxygenisotopes in R-chondrite magnetite and olivine: Links between $\mathrm{R}$ chondrites and ordinary chondrites. Geochimica et Cosmochimica Acta 64, 3897- 3911.

212. Greenwood J. P., Wasson J. T., and McKeegan K. D. (2000a) Metamorphosed $\mathrm{CAl}$ in Karoonda: Constraints on CK metamorphism from oxygen isotopic compositions. 31st Lunar and Planetary Science Conference, \#2042 (abstract).

213. Gregory T., Zolensky M. E., Trieman A., Berger E., Le L., Fagan A., Takenouchi A., Velbel M.A., and Nishiizumi K. (2015) Lithologies Making Up CM Carbonaceous Chondrites and Their Link to Space Exposure Ages. 46th Lunar and Planetary Science Conference, Abstract \#1227.

214. Greshake A., Clayton R. N., Mayeda T. K. and Kurz M. (2003) NWA 1465 and NWA 1665: two unusual carbonaceous chondrites from Northwest Africa. 34th Lunar and Planetary Science Conference, \#1560 (abstract).

215. Greshake A., Krot A. N., Meibom A., Weisberg M. K., Zolensky M., and Keil K. (2002) Heavily-hydrated matrix lumps in the $\mathrm{CH}$ and metal-rich chondrites QUE 94411 and Hammadah Al Hamra 237. Meteoritics and Planetary Science 37, 281-293.

216. Grossman J. N. and Brearley A. J. (2005) The onset of metamorphism in ordinary and carbonaceous chondrites. Meteoritics \& Planetary Science, 40, 87-122.

217. Grossman J. N., Rubin A. E., Nagahara H. and King E. A. (1988) Properties of chondrules. In Meteorites and the Early Solar System (ed. Kerridge J. F. and Matthews M. S.) pp. 619-659. Univ. Arizona Press, Tucson.

218. Grossman J. N. and Wasson J. T. (1985). The origin and history of the metal and sulfide components of chondrules. Geochimica et Cosmochimica Acta 49:925-939.

219. Grossman L. (1975) Petrography and mineral chemistry of Ca-rich inclusions in the Allende meteorite. Geochim. Cosmochim Acta 39, 433-454.

220. Grossman L. (1980) Refractory inclusions in the Allende meteorite. Ann. Rev. Earth Planet. Sci. 8, 559-608.

221. Grossman L. and Ganapathy R. (1976) Traqce elements in the Allende meteorite -- II. Fine-grained, Ca-rich inclusions. Geochim. Cosmochim. Acta 40, 967-977.

222. Grossman L. and Olsen, E. (1974). Origin of the high-temperature fraction of C2 chondrites. Geochimica et Cosmochimica Acta 38, 173.

223. Grossman L. and Steele I.M. (1976) Amoeboid olivine aggregates in the Allende meteorite. Geochim. Cosmochim. Acta 40, 149-155.

224. Grove T. L., Ferry J. M., and Spears F. S. (1983) Phase transitions and decomposition relations in calcic plagioclase. American Mineralogist 68, 41-59.

225. Haack H., Grau T., Bischoff A., Horstmann M., Wasson J., Sørensen A., Laubenstein M., Ott U., Palme H., Gellissen M., Greenwood R., Pearson V., Franchi I., 
Gabelica Z., Schmitt-Kopplin P. (2012) Maribo-A new CM fall from Denmark. Meteoritics and Planetary Science 47, 30-50.

226. Han J. and Brearley A. J. (2016). Microstructural constraints on complex thermal histories of refractory CAl-like objects in an amoeboid olivine aggregate from the ALHA77307 CO3.0 chondrite. Geochimica et Cosmochimica Acta, 183, 176-197.

227. Hanna R. D. and Ketcham R.A. (2018) Evidence for accretion of fine-grained rims in a turbulent nebula for CM Murchison. Earth and Planetary Science Letters 481, 201211.

228. Hanna R. D., Ketcham R. A., Zolensky M. and Behr W. (2015) Impact-induced brittle deformation, porosity loss, and aqueous alteration in the Murchison CM chondrite. Geochimica et Cosmochimica Acta 171, 256-282.

229. Hanowski N. P. and Brearley A. J. (2001) Aqueous alteration of chondrules in the CM carbonaceous chondrite, Allan Hills 81002: Implications for parent body alteration. Geochimica et Cosmochimica Acta 65, 495-518.

230. Harju, E.R., Rubin, A.E., Ahn, I., Choi, B.-G., Ziegler, K., and Wasson, J.T. (2014) Progressive aqueous alteration of CR carbonaceous chondrites. Geochimica et Cosmochimica Acta 139, 267-292.

231. Harker R. I. and Tuttle O. F. (1956) The lower stability limit of akermanite. American Journal of Science 254, 468-478.

232. Harries D. and Zolensky M. E. (2016). Mineralogy of iron sulfides in CM1 and Cl1 lithologies of the Kaidun breccia: Records of extreme to intense hydrothermal alteration. Meteoritics \& Planetary Science 51, 1096-1109.

233. Hashimoto A. and Grossman L. (1987) Alteration of Al-rich inclusions inside amoeboid olivine aggregates in the Allende meteorite. Geochimica et Cosmochimica Acta 51, 1685-1704.

234. Henke S., Gail H.-P., Trieloff M., and Schwartz W. H. (2013) Thermal evolution model for the $\mathrm{H}$ chondrite asteroid- instantaneous formation versus protracted accretion. Icarus 226, 212-228.

235. Herd C., Hilts R., Skelhorne A., Simkus D. (2016) Cold curation of pristine astromaterials: Insights from the Tagish Lake meteorite. Meteoritics \& Planetary Science 51, 499-519.

236. Herzog, G.F. (2003) Cosmic-ray exposure ages of meteorites, in Davis, A.M., ed., Meteorites, Comets, and Planets, Holland, H.D., and Turekian, K.K., eds., Treatise on Geochemistry, vol. 1 p. 347-380.

237. Herzog G. F. and Caffee M. W. (2014) Cosmic-Ray Exposure Ages of Meteorites. In Treatise on Geochemistry (Heinrich Holland and Karl Turekian eds), 419-454.

238. Hewins R. H., Connolly, Jr., H. C., Lofgren G. E. and Libourel G. (2005) Experimental constraints on chondrule formation. In Chondrites and the Protoplanetary Disk (eds. A. N. Krot, E. R. D. Scott and B. Reipurth), pp. 286-316. ASP Conference Series 341. Astronomical Society of the Pacific, San Francisco.

239. Hewins R. H., Bourot-Denise, M., Zanda, B., Leroux, H., Barrat, J.-A., Humayan, M., Göpel, G., Greenwood, R.C., Franchi, I.A., Pont, S., Lorand, J.-P., Cournède, Gatacceca, J., Rochette, P., Kuga, M., Marrocchi, Y., and Marty, B. (2014) The Paris meteorite, the least altered CM chondrite so far. Geochim. Cosmochim. Acta 124, 190222.

240. Hewins, R. H. and Zanda, B. (2012) Chondrules: Precursors and interactions with the nebular gas. Meteoritics \& Planet. Sci. 47, 1120-1138. 
241. Heymann D. (1967) On the origin of hypersthene chondrites: Ages and shock effects of black chondrites. Icarus 6, 189-221.

242. Hezel D. C., Russell S. S., Ross A. J. and Kearsley A. T. (2008) Modal abundances of CAls: implications for bulk chondrite element abundances and fractionations. Meteorit. Planet. Sci. 43, 1879-1894.

243. Hinton R. W., Davis A. M., Scatena-Wachel D. E., Grossman L., and Draus R. J. (1988) A chemical and isotopic study of hibonite-rich refractory inclusions in primitive meteorites. Geochim. Cosmochim. Acta 52, 2573-2598.

244. Hirata N., Barnouin-Jha O. S., Honda C., et al., (2009) Survey of possible impact structured on 25143 Itokawa. Icarus 200, 486-502.

245. Hiroi T., Zolensky, M.E., and Pieters, C.M. (2001) The Tagish Lake meteorite: A possible sample from a D-type asteroid, Science 293, 2234-2236.

246. Hiyagon H., Nakamura T., and Nakamura N. (2001) An ion microprobe study of oxygen isotopes in the Kobe CK4 meteorite. Antarct. Meteorites 26, 35-37.

247. Hiyagon H., Sugiura N., Kita N. T., Kimura M., Morishita Y., Takehana Y. (2016). Origin of the eclogitic clasts with graphite-bearing and graphite-free lithologies in the Northwest Africa 801 (CR2) chondrite: Possible origin from a Moon-sized planetary body inferred from chemistry, oxygen isotopes and REE abundances. Geochimica et Cosmochimica Acta 186, 32-48.

248. Holmberg A. A. and Hashimoto A. (1992). A unique, (almost) unaltered spinel-rich fine-grained inclusion in Kainsaz. Meteoritics 27:149-153.

249. Hohenberg C. M., Meshik A. P., Pravdivtseva O. V. and Krot A. N. (2001) I-Xe dating: Dark inclusions from Allende CV3 (abstract). Meteorit. Planet. Sci. 36, A83.

250. Hoppe, P., Macdougall, D., Lugmair, G.W. (2007) High spatial resolution ion microprobe measurements refine chronology of carbonate formation in Orgueil. Meteorit. Planet. Sci. 42 (7/8), 1309-1320.

251. Horstmann M. and Bischoff A. (2014). he Almahata Sitta polymict breccia and the late accretion of asteroid 2008 TC3. Chemie der Erde - Geochemistry 74, 149-183.

252. Howard K. T., Alexander C. M. O'D., and Dyl, K. A. (2014). PSD-XRD Modal mineralogy of type $3.0 \mathrm{CO}$ Chondrites: Initial asteroidal water mass fractions and implications for CM chondrites (abstract). LPI Contribution No. 1777, p.1830

253. Howard K. T., Benedix G. K., Bland P. A. and Cressey G. (2009) Modal mineralogy of CM2 chondrites by X-ray diffraction (PSD-XRD). Part 1: Total phyllosilicate abundance and the degree of aqueous alteration. Geochimica et Cosmochimica Acta 73, 4576-4589.

254. Howard K. T., Benedix G. K., Bland P. A. and Cressey G. (2011) Modal mineralogy of $\mathrm{CM} 2$ chondrites by X-ray diffraction (PSD-XRD). Part 2: Degree, nature and settings of aqueous alteration. Geochimica et Cosmochimica Acta 75:, 2735-2751.

255. Howard K. T., Alexander C. M. O'D., Schrader D. L., and Dyl K. A. (2015a). Classification of hydrous meteorites (CR, CM and $\mathrm{C} 2$ ungrouped) by phyllosilicate fraction: PSD-XRD modal mineralogy and planetesimal environments. Geochimica et Cosmochimica Acta 149, 206-222.

256. Howard K. T., Davidson J., Alexander C. M. O'D., and Abreu N. M. ( 2015b). The glue that holds worlds together: Abundance, origin and significance of amorphous $\mathrm{Fe}, \mathrm{Mg}$ silicate in carbonaceous chondrites (abstract). 46th LPSC No. 1832, p.2244.

257. Hsu W., Guan Y., Hua X., Wang Y., Leshin L. A., and Sharp T. G. (2006) Aqueous alteration of opaque assemblages in the Ningqiang carbonaceous chondrite: Evidence from opaque assemblages in oxygen isotopes. Earth and Planetary Science Letters 243, 107-114. 
258. Huang J., Ji J., Ye Peijian., et al. (2013) The Ginger-shaped Asteroid 4179 Toutatis: New observations from a successful flyby of Chang'e-2. Scientific Reports 3, 3411.

259. Hubert H., Rubin A. E., Kallemeyn G. W., and Wasson J. T. (2006) Siderophileelements anomalies in CK carbonaceous chondrites : Implications for parent-body aqueous alteration and terrestrial weathering of sulfides. Geochimica et Cosmochimica Acta 70, 4019-4037.

260. Hurt S.M., Rubin A.E. and Wasson J.T. (2012) Fractionated matrix composition in CV3 Vigarano and alteration processes on the CV parent asteroid. Meteorit. Planet. Sci. 47, 1035-1048.

261. Huss G. R. (1990) Ubiquitous interstellar diamond and SiC in primitive chondrites: Abundances reflect metamorphism. Nature 347, 159-162.

262. Huss G. R. and Lewis R. S. (1994a) Noble gases in presolar diamonds I: Three distinct components and their implications for diamond origins. Meteorit. Planet. Sci. 29, 791-810.

263. Huss G. R. and Lewis R. S. (1994b) Noble gases in presolar diamonds II: Component abundances reflect thermal processing. Meteorit. Planet. Sci. 29, 811-829.

264. Huss G. R., Rubin A. E. and Grossman J. N. (2006) Thermal metamorphism in chondrites. Meteorites and the early solar system II, 567-586.

265. Hutcheon I. D. and Newton R. C. (1981) Mg isotopes, mineralogy, and mode of formation of secondary phases in C3 refractory inclusions. 12th Lunar and planetary Science. pp. 491-493 (abstract).

266. Hutchison R. (2004) Meteorites: A Petrologic, Chemical and Isotopic Synthesis. Cambridge University Press, Cambridge, U.K. 506 pp.

267. Hutchison R., Alexander C., and Barber D. (1987) The Semarkona meteorite: First recorded occurrence of smectite in an ordinary chondrite, and its implications. Geochimica et Cosmochimica Acta 51, 1875-1882.

268. Humayun M., Campbell A. J., Zanda, B., and Bourot-Denise, M. 2002, 33rd LPSC No. 1965.

269. Ibrahim, E.-M.I., 2012. The Elastic Properties of Carbonaceous Chondrites M.Sc. Thesis. University of Calgary, Alberta, Canada.

270. Ikeda Y. (1982). Petrology of the ALH-77003 chondrite (C3). In: Proceedings of the 7th symposium on Antarctic meteorites. Memoirs National Institute of Polar Research special issue, vol 25. Tokyo, pp 34-65.

271. Ikeda, Y. (1991). Petrology and mineralogy of the Yamato-82162 chondrite (Cl). Proc. NIPR Symp. Antarct. Meteorites 4, 187-225.

272. Ikeda Y. and Noguchi T. and Kimura M. (1992) Petrology and mineralogy of the Yamato-86720 carbonaceous chondrite. Proc. Nipr Symp. Antarct. Meteorites 5, 136-154.

273. Ikornikova, A., Sheptunov, D., 1973. Dissociation curves of trigonal carbonates. Crystallization Processes Under Hydrothermal Conditions (Edited by A.N. Lobachev). New York, Consultants Bureau, 113-123.

274. Ireland T. R. (1988) Correlated morphological, chemical, and isotopic characteristics of hibonites from the Murchison carbonaceous chondrite. Geochim. Cosmochim. Acta 52, 2827-2839.

275. Ireland T. R. (1990) Presolar isotopic and chemical signatures in hibonite-bearing refractory inclusions from the Murchison carbonaceous chondrite. Geochim. Cosmochim. Acta 54, 3219-3237. 
276. Ireland T. R., Fahey A. J., and Zinner E. K. (1988) Trace element abundances in hibonites from the Murchison carbonaceous chondrite: constraints on high-temperature processes in the solar nebula. Geochim. Cosmochim. Acta 52, 2841-2854.

277. Isa M., Shirai N., Ebihara M., Kubuki S., and Yamaguchi A. (2011) Chemical characteristics for CK carbonaceous chondrites. 42nd Lunar and Planetary Science Conference, \#1876 (abstract).

278. Ito M., Nagasawa H., and Yurimoto H. (2004). Oxygen isotopic SIMS analysis in Allende CAl: details of the very early thermal history of the solar system. Geochimica et Cosmochimica Acta, 68, 2905-2923.

279. Itoh D. and Tomeoka K. (1998). Na-bearing Ca-Al-rich inclusions in four CO3 chondrites, Kainsaz, Ornans, Lancé, and Warrenton (abstract). Symp Antarct Meteorites 23, 42-44.

280. Itoh D. and Tomeoka K. (2000). Dark Inclusions in CO3 Chondrites: How are They Related to Dark Inclusions in CV3 Chondrites? (abstract). Meteoritics \& Planetary Science, vol. 35, Supplement, p.A81.

281. Itoh D. and Tomeoka K. (2003). Dark inclusions in CO3 chondrites: new indicators of parent-body processes. Geochimica et Cosmochimica Acta, 67, 153-169

282. Itoh S., Kojima H., and Yurimoto H. (2004). Petrography and oxygen isotopic compositions in refractory inclusions from $\mathrm{CO}$ chondrites. Geochimica et Cosmochimica Acta, 68, 183-194.

283. Ivanov A. V., MacPherson G. J., Zolensky M. E., Kononkova N. N., and Migdisova L. F. (1996). The Kaidun meteorite: Composition and origin of inclusions in the metal of an enstatite chondrite clast. Meteoritics \& Planetary Science 31, 621-626.

284. Ivanova M., Kononkova N., Krot A., Greenwood R., Franchi I., Verchovsky A., Trieloff M., Korochantseva E., Brandstätter F. (2008) The Isheyevo meteorite: Mineralogy, petrology, bulk chemistry, oxygen, nitrogen, carbon isotopic compositions, and 40Ar-39Ar ages. MAPS 43, 915-940.

285. Ivanova M. A., Nazarov A., Kononkova N. N., Taylor L. A., Patchen A., Clayton R. N. and Mayeda T. K. (2000) Dhofar 015, a new CK3 chondrite: a record of nebular processes. Meteorites and Planetary Science 35, A83 (abstract).

286. Ivanova M. A., Nazarov M. A., Clayton R. N., Mayeda T. K. and Taylor L. A. (2003) Sayh al Uhaymir 085, CV chondrite: mineralogical links with CK chondrites. 34th Lunar and Planetary Science Conference, \#1226 (abstract).

287. Jacobsen B., Yin Q.-Z., MOynier F., Amelin Y., Krot A. N., Nagahima K., Hutcheon I. D. and Palme H. (2008) 26Al-26Mg and 207Pb-206Pb systematics of Allende CAls: Canonical solar initial 26Al/27ASI ratio reinstated. Earth Planet. Sci. Lett. 272, 353-364.

288. Jacquet E., Fromang S. and Gounelle M. (2011) Radial transport of refractory inclusions and their preservation in the dead zone. Astron. \& Astrophys. 526, L8.

289. Jarosewich E., Clarke R. S. and Barrows J. N. (1987) The Allende meteorite reference sample. Smithsonian Contrib. Earth Sci. 27, 1-49.

290. Jenniskens P., Fries M. D., Yin Q.-Z., Zolensky M., Krot, A. N., Sandford S. A., Sears D., Beauford R., Ebel D. S., Friedrich J. M., Nagashima K., Wimpenny J., Yamakawa A., Nishiizumi K., Hamajima Y., Caffee M. W., Welten K. C., Laubenstein M., Davis A. M., Simon S. B., Heck P. R., Young E. D., Kohl I. E., Thiemens M. H.; Nunn M. H., Mikouchi T., Hagiya K., Ohsumi K., Cahill T. A., Lawton J. A., Barnes D., Steele A., Rochette P., Verosub K. L., Gattacceca J. Cooper G., Glavin D. P., Burton A. S., Dworkin J. P., Elsila J. E., Pizzarello S., Ogliore R., Schmitt-Kopplin P., Harir M., Hertkorn N., Verchovsky A., Grady M., Nagao K., Okazaki R., Takechi H., Hiroi T., Smith K., Silber E. 
A., Brown P. G., Albers J., Klotz D., Hankey M., Matson R., Fries J. A.; Walker R. J.; Puchtel I., Lee C.-T. A., Erdman M. E., Eppich G. R., Roeske S., Gabelica Z., Lerche M., Nuevo M.,Girten B., and Worden S. P. (2012). Radar-Enabled Recovery of the Sutter's Mill Meteorite, a Carbonaceous Chondrite Regolith Breccia. Science, 338, 1583-

291. Jiang Y., Ji J., Huang J., et al. (2015) Boulders on asteroid Toutatis as observed by Chang'e-2. Scientific Reports 5, 16029.

292. Johnson, C. A., Prinz, M. (1993) Carbonate compositions in CM and Cl chondrites, and implications for aqueous alteration. Geochimica et Cosmochimica Acta 57 (12), 2843-2852.

293. Johnson C. A., Prinz M., Weisberg M.K., Clayton R.N. and Mayeda T.K. (1990) Dark inclusions in Allende, Leoville, and Vigarano: Evidence for nebular oxidation of CV3 constituents. Geochimica et Cosmochimica Acta 54, 819-831.

294. Johnson J., Zolensky M., Chan Q., Kring D. (2016) Intriguing dehydrated phyllosilicates found in an unusual clast in the LL3.15 chondrite NWA6925. 47th Lunar and Planetary Science Conference. Abstract.

295. Johnson J., Zolensky M., Chan Q, Kring D. (2015) Striking Graphite Bearing Clasts Found in Two Ordinary Chondrite Samples; NWA6169 \& NWA8330. GSA 2015 Abstracts with Program.

296. Jones R. H. and Rubie D. C. (1991). Thermal histories of CO3 chondrites application of olivine diffusion modelling to parent body metamorphism. Earth and Planetary Science Letters, 106, 73-86.

297. Jull A. J. T., Cheng S., Gooding J. L., and Velbel M. A. 1988. Rapid growth of magnesium-carbonate weathering products in a stony meteorite from Antarctica. Science 242:417-419.

298. Kallemeyn G. W., Boynton W. V., Willis J., and Wasson J. T. (1978). Formation of the Bencubbin polymict meteoritic breccia. Geochimica et Cosmochimica Acta 42, 507515.

299. Kallemeyn G. W., Rubin A. E., Wang D., and Wasson J. T. (1989) Ordinary chondrites: Bulk compositions, classification, lithophile-element fractionations, and composition-petrographic type relationships. Geochimica and Cosmochimica Acta 53, 2747-2767.

300. Kallemeyn G. W., Rubin A. E., and Wasson J. T. (1991). The compositional classification of chondrites: V - The Karoonda (CK) group of carbonaceous chondrites. Geochimica et Cosmochimica Acta 55, 881-892.

301. Kallemeyn G. W., Rubin A. E., and Wasson J. T. (1994). The compositional classification of chondrites: VI. The CR carbonaceous chondrite group. Geochimica et Cosmochimica Acta 58, 2873-2888.

302. Kallemeyn G. W., Rubin A. E., and Wasson J. T. (1991). The compositional classification of chondrites: V. The Karoonda (CK) group of carbonaceous chondrite. Geochimica et Cosmochimica Acta 55, 881-892.

303. Kallemeyn G. W. and Wasson J. T. (1981). The compositional classification of chondrites-I. The carbonaceous chondrite groups. Geochimica et Cosmochimica Acta, 45, 1217-1230.

304. Kallemeyn G. W. and Wasson J. T. (1982). The compositional classification of chondrites: III. Ungrouped carbonaceous chondrites. Geochimica et Cosmochimica Acta 46, 2217-2228.

305. Kaluna H. M., Masiero J. R., and Meech K. J. (2016) Space weathering trends among carbonaceous asteroids. Icarus $264,62-71$. 
306. Keil K. (1989) Enstatite meteorites and their parent bodies. Meteoritics 24, 195208.

307. Keil K. (1968) Mineralogical and chemical relationships among enstatite chondrites. Journal of Geophysical Research 73, 6945-6976.

308. Keller L. P. (1992) Petrography and mineral chemistry of calcium-aluminum-rich inclusions in the Maralinga CK4 chondrite. 23rd Lunar and Planetary Science Conference, pp. 671-672 (abstract).

309. Keller L. P. (1993) Heterogeneous plagioclase compositions in the Maralinga CK4 chondrite. 24th Lunar and Planetary Science Conference, pp. 783-784 (abstract).

310. Keller L. P. and Berger E. L. (2014) A transmission electron microscope study of Itokawa regolith grains. Earth, Planets and Space 66, article \#71.

311. Keller, L. P. and Buseck, P. R. (1990a). Matrix mineralogy of the Lancé CO3 carbonaceous chondrite: A transmission electron microscope study. Geochimica et Cosmochimica Acta, 54, 1155-1163.

312. Keller L. P. and Buseck P. R. (1990b). Aqueous alteration in the Kaba CV3 carbonaceous chondrite. Geochimica et Cosmochimica Acta 54:2113-2120.

313. Keller L. P. and Buseck P. R. (1991). Calcic micas in the Allende meteorite: Evidence for hydration reactions in the early solar nebula. Science 252:946-949.

314. Keller L. P., Christoffersen R., Dukes C. A., Baragiola R. A., and Rahman Z. (2015). Ion irradiation experiments of the Murchison CM2 carbonaceous chondrite: Simulating space weathering of primitive asteroids. LPSC XLVI, abstract \#1913.

315. Keller L. P., Clark J. C., Lewis C. F., and Moore C. B. (1992) Maralinga, a metamorphosed carbonaceous chondrite found in Australia. Meteoritics 27, 87-91.

316. Keller L. P., Thomas K. L., Clayton R. N., Mayeda T. K., DeHart J. M., and McKay D. S. (1994). Aqueous alteration of the Bali CV3 chondrite: Evidence from mineralogy, mineral chemistry, and oxygen isotopic compositions. Geochimica et Cosmochimica Acta, 5589-5598.

317. Kimura M., Grossman J. N., and Weisberg M. K. (2011). Fe-Ni metal and sulfide minerals in $\mathrm{CM}$ chondrites: An indicator for thermal history. Meteoritics \& Planetary Sciences 46, 431-442.

318. Kimura M., Grossman J. N., and Weisberg M. K. (2008). Fe-Ni metal in primitive chondrites: Indicators of classification and metamorphic conditions for ordinary and CO chondrites. Meteoritics \& Planetary Science 43,1161-1177.

319. Kimura M. and Ikeda Y. (1997) Comparative study of anhydrous alteration of chondrules in reduced and oxidized CV chondrites. Antarct. Meteorite Res. 10, 191-202.

320. King A., Schofield P., Howard K., Russell S. (2015) Modal mineralogy of Cl and Cl-like chondrites by X-ray diffraction. Geochimica et Cosmochimica Acta 165, 148-160.

321. Kojima T., Yada S., and Tomeoka K. (1995). Ca-Al-rich inclusions in three Antarctic CO3 chondrites, Yamato-81020, Yamato-820050 and Yamato-790992: record of low-temperature alteration. Proceeding of the NIPR Symposium in Antarctic Meteorites 8:79-96.

322. Komatsu M., Krot A. N., Petaev M. I., Ulyanov A. A., Keil K., Miyamoto M. (2001) Mineralogy and petrography of amoeboid olivine aggregates from the reduced CV3 chondrites Efremovka, Leoville, and Vigarano: Products of nebular condensation, accretion, and annealing. Meteorit. Planet. Sci. 36, 629-641.

323. Komatsu M. (2003) Mineralogical study of amoeboid olivine aggregates in CV3 chondrites: Implications for their origin and relation to chondrules. Ph.D. thesis, Univ. Tokyo, Tokyo, Japan. 197 p. 
324. Kornacki A.S. and Wood J. A. (1984) Petrography and classification of Ca,Al-rich and olivine-rich inclusions in the Allende CV3 chondrite. Proceedings Lunar and Planetary Science Conference 14, B573-B587.

325. Kornacki A. S., Cohen R. E. and Wood J. A. (1983) Petrography and classification of refractory inclusions in the Allende and Mokoia CV3 chondrites. Proc. 8th Symp. Antarct. Meteorit. pp. 45-60.

326. Kracher A., Keil K., Kallemeyn G. W., Wasson J. T. and Clayton R. N. (1985) The Leoville (CV3) accretionary breccia. Proc. Lunar Planet. Sci. Conf. 16th, D123-D135.

327. Krot A. N., Amelin Y., Cassen P., and Meibom A. 2005. Young chondrules in CB chondrites from a giant impact in the early solar system. Nature 436, 989-992.

328. Krot A. N. and Keil K. (2002). Anorthite-rich chondrules in CR and $\mathrm{CH}$ carbonaceous chondrites: genetic link between calcium-aluminum-rich inclusions and ferromagnesian chondrules. Meteoritics \& Planetary Science 37, 91-111.

329. Krot A. N., Keil K., Goodrich C. A., Scott E. R. D., and Weisberg M. K. (2003). Classification of meteorites. In Meteorites, comets, and planets, edited by Davis A. M., Treatise in Geochemistry (Holland H. D. and Turekian K. K., series editors), Volume 1. Oxford: Elsevier-Pergamon. pp. 83-128.

330. Krot A. N., Libourel G. and Chaussidon M. (2006b) Oxygen isotope compositions of chondrules in CR chondrites. Geochimica et Cosmochimica Acta 70, 767-779.

331. Krot A. N., McKeegan K. D., Leshin L. A., MacPherson G. J., and Scott E. R. D. (2002) Existence of an ${ }^{16}$ O-rich gaseous reservoir in the solar nebula. Science 295, 10511054.

332. Krot A. N., Meibom A., Russell S. S., Alexander C. M. O'D., Jeffries T. E., and Keil K. (2001) A new astrophysical setting for chondrule formation. Science 291, 1776-1779.

333. Krot A. N., Meibom A., Russell S. S., Alexander C. M. O'D., Jeffries T. E., and Keil K. (2001) A new astrophysical setting for chondrule formation. Science 291, 1776-1779.

334. Krot A. N. and Nagashima K. (2017) Constraints on mechanisms of chondrule formation from chondrule precursors and chronology of transient heating events in the protoplanetary disk. Geochemical Journal 51, 45-68.

335. Krot A. N., Nagashima K., Jacobsen B., Hutcheon I. D., Ishii H., Yin Q.-Z., Davis A. M., and Simon S. B. (2009) Origin of grossular-bearing assemblages in CAls from CV carbonaceous chondrites. Meteoritics \& Planetary Science 44, A5353 (abstract).

336. Krot A. N., Nagashima K., Yoshitake M. and Yurimoto H. (2010). Oxygen isotopic compositions of chondrules from the metalrich chondrites Isheyevo $(\mathrm{CH} / \mathrm{CBb}), \mathrm{MAC}$ 02675 (CBb) and QUE 94627 (CBb). Geochimica et Cosmochimica Acta 74, 2190-2211.

337. Krot A. N., Petaev M.I., Scott E. R. D., Choi B.-G., Zolensky M. E. and Keil K. (1998) Progressive alteration in CV3 chondrites: More evidence for asteroidal alteration. Meteorit. Planet. Sci. 33, 1065-1085.

338. Krot A. N., Petaev M. I., Russell S. S., Itoh S., Fagan T. J., Yurimoto H., Chizmadia L., Weisberg M. K., Komatsu M. and Ulyanov A. A. (2004a) Amoeboid olivine aggregates and related objects in carbonaceous chondrites: records of nebular and asteroid processes. Chemie der Erde-Geochemistry 64, 185-239.

339. Krot A. N., Scott E. R. D. and Zolensky M. E. (1995) Mineralogical and chemical modification of components in CV3 chondrites: Nebular or asteroidal processing? Meteoritics 30, 748-775.

340. Krot A., Scott E. R. D. and Zolensky M. (1997) Origin of fayalitic olivine rims and lath-shaped matrix olivine in the CV3 chondrite Allende and its dark inclusions. Meteoritics and Planetary Science 32, 31-49. 
341. Krot A. N., Yurimoto H., Hutcheon I. D., Chaussidon M., MacPherson G. J., and Paque J. (2007). Remelting of refractory inclusions in the chondrule-forming regions: Evidence from chondrule-bearing type $\mathrm{C}$ calcium-aluminum-rich inclusions from Allende. Meteoritics \& Planetary Science 42, 1197-1219.

342. Krot A. N., Yurimoto H., Hutcheon I. D., Libourel G., Chaussidon M., Tissandier L., Petaev M. I., MacPherson G. J., Paque-Heather J. and Wark D. (2007). Type C Ca,Al-rich inclusions from Allende: Evidence for multistage formation. Geochim. Cosmochim. Acta 71, 4342-4364.

343. Krot A. N., Yurimoto H., McKeegan K. D., Leshin L., Chaussidon M., Libourel G., Yoshitake M., Huss G. R., Guan Y. and Zanda B. (2006a). Oxygen isotopic compositions of chondrules: implications for evolution of oxygen isotopic reservoirs in the inner solar nebula. Chemie der Erde - Geochemistry, 66, 249-276.

344. Krot A. N. and Wasson J. T. (1995) Igneous rims on low-FeO and high-FeO chondrules in ordinary chondrites. Geochimica et Cosmochimica Acta 59, 4951-4966.

345. Krot A. N., Zolensky M. E., Wasson J. T., Scott E. R. D., Keil K., and Ohsumi K. (1997) Carbide-magnetite-bearing type-3 ordinary chondrites. Geochimica et Cosmochimica Acta 61, 219-237.

346. Kruijer T. S., Burkhardt C., Budde G. and Kleine T. (2017a) Age of Jupiter inferred from the distinct genetics and formation times of meteorites. Proc. Nat. Acad. Sci. 114, 6712-6716.

347. Kruijer T. S., Kleine T., Burkhardt C. and Budde G. (2017b) Dating the formation of Jupiter through $\mathrm{W}$ and Mo isotope analyses of meteorites. Lunar Planet. Sci. 48, abstract\#1386.

348. Kruijer T. S., Burkhardt C., Budde G. and Kleine T. (2017c) Dating the formation of Jupiter using the distinct genetics and formation times of meteorites. Ann. Mtg. Meteoritical Soc., abstract\#6333.

349. Kunihiro T., Rubin A. E., McKeegan K. D., and Wasson J. T. (2004). Initial 26Al/27Al in carbonaceous chondrite chondrules: too little 26Al to melt asteroids. Geochimica et Cosmochimica Acta, 68, 2947-2957.

350. Kurahashi E, Kita N. T., Nagahara H., and Morishita Y. (2008). 26Al-26Mg systematics of chondrules in a primitive $\mathrm{CO}$ chondrite. Geochimica et Cosmochimica Acta 72, 3865-3883.

351. Kurat G., Palme H., Brandstätter F. and Huth J. (1989) Allende xenolith AF: Undisturbed record of condensation and aggregation of matter in the solar nebula. $Z$. Naturforsch. 44a, 988-1004.

352. Kurat G., Zinner E., and Brandstätter F. (2002) A plagioclase-olivine-spinelmagnetite inclusion from Maralinga (CK): Evidence for sequential condensation and solidgas exchange. Geochimica et Cosmochimica Acta, 66, 2959-2979.

353. Lange M. A., Lambert P. and Ahrens T. J. (1985) Shock effects on hydrous minerals and implications for carbonaceous chondrites. Geochim. Cosmochim. Acta 49, 1715-1726.

354. Langenhorst F., Harries D., Pollok K., and van Aken P. A. (2014) Mineralogy and defect microstructure of an olivine-dominated Itokawa dust particle: evidence for shock metamorphism, collisional fragmentation, and LL chondrite origin. Earth, Planets and Space 66, article \#118.

355. Lee M. R. and Bland P. A. (2004). Mechanisms of weathering of meteorites recovered from hot and cold deserts and the formation of phyllosilicates. Geochimica et Cosmochimica Acta 68:893-916. 
356. Lee M. R., and Lindgren P. (2016) Aqueous alteration of chondrules from the Murchison CM carbonaceous chondrite: Replacement, pore filling, and the genesis of polyhedral serpentine. Meteoritics \& Planetary Science, 51, 1003-1021.

357. Lee M., Nicholson K. (2009) Ca-carbonate in the Orgueil (Cl) carbonaceous chondrite: Mineralogy, microstructure and implications for parent body history. Earth and Planetary Science Letters 280 (2009) 268-275.

358. Lee M. S., Rubin A. E., and Wasson, John T. Origin of metallic Fe-Ni in Renazzo and related chondrites. Geochimica et Cosmochimica Acta 56, 2521-2533.

359. Lee P., Veverka J., Thomas P. C., et al., (1996) Ejecta blocks on 243 Ida on other asteroids. Icarus 120, 87-105.

360. Lee T., Papanastassiou D. A., and Wasserburg G. J. (1976) Demonstration of ${ }^{26} \mathrm{Mg}$ excess in Allende and evidence for ${ }^{26} \mathrm{Al}$. Geophysical Research Letters 3: 109-112.

361. Leroux H., Rietmeijer F. J. M., Velbel M. A., Brearley A. J., Jacob D., Langenhorst F., Bridges J. C., Zega T. J., Stroud R. M., Cordier P., Harvey R. P., Lee M., Gounelle M. and Zolensky M. E. (2008) A TEM study of thermally modified Comet 81P/Wild 2 dust particles by interactions with the aerogel matrix during the Stardust capture process. Meteoritics \& Planetary Science, 43, 97-120.

362. Leroux H., Cuvillier P., Zanda B., and Hewins R.H. (2015) GEMS-like material in the matrix of the Paris meteorite and the early stages of alteration of $\mathrm{CM}$ chondrites. Geochim. Cosmochim. Acta 170, 247-265.

363. Le Guillou C. and Brearley A. J. (2014). Relationships between organics, water and early stages of aqueous alteration in the pristine CR3.0 chondrite MET 00426. Geochimica et Cosmochimica Acta, 131, 344-367.

364. Le Guillou C., Bernard S., Brearley A. J., and Remusat L. (2014). Evolution of organic matter in Orgueil, Murchison and Renazzo during parent body aqueous alteration: In situ investigations. Geochimica et Cosmochimica Acta, 131, 368-392.

365. Le Guillou C., Changela H. G., and Brearley A. J. (2015a). Widespread oxidized and hydrated amorphous silicates in CR chondrites matrices: Implications for alteration conditions and $\mathrm{H}_{2}$ degassing of asteroids. Earth and Planetary Science Letters, 420, 162173.

366. Le Guillou C., Dohmen R., Rogalla D., Müller T., Vollmer C., Becker H.-W. (2015b). New experimental approach to study aqueous alteration of amorphous silicates at low reaction rates. Chemical Geology, 412, 179-192.

367. Libourel G. and Chaussidon M. (2011) Oxygen isotopic constraints on the origin of Mg-rich olivines from chondritic meteorites. Earth and Planetary Science Letters 301, 921.

368. Lindgren P. and Lee M .R. (2015) Tracking the earliest stages of aqueous alteration in the mildly altered CM chondrite EET 96029 (abstract \#1760). 46th Lunar and Planetary Science Conference. CD-ROM.

369. Lindgren P., Lee M. R., Sofe M., and Zolensky, M. E. (2013) Clasts in the CM2 carbonaceous chondrite Lonewolf Nunataks 94101: Evidence for aqueous alteration prior to complete mixing. Meteoritics \& Planetary Science, 48, 1074-1090.

370. Lindgren P., Hanna R. D., Dobson K. J., Tomkinson T., and Lee M. R. (2015) The paradox between low shock-stage and evidence for compaction in CM carbonaceous chondrites explained by multiple low-intensity impacts. Geochimica et Cosmochimica Acta 148, 159-178.

371. Lodders K., Fegley B. (1998) The Planetary Scientist's Companion. Univ. Oxford Press. 
372. Loeffler M. J., Dukes C. A., and Baragiola R. A. (2009). Irradiation of olivine by 4 $\mathrm{keV} \mathrm{He+:} \mathrm{Simulation} \mathrm{of} \mathrm{space} \mathrm{weathering} \mathrm{by} \mathrm{solar} \mathrm{wind.} \mathrm{Journal} \mathrm{of} \mathrm{Geophysical} \mathrm{Research}$ 114: E03003, doi:10.1029/2008JE003249

373. Losiak A. and Velbel M. A. (2011) Evaporite formation during weathering of Antarctic meteorites -- A weathering census analysis based on the ANSMET database. Meteoritics \& Planetary Science 46, 443-458.

374. Lunning N. G., Corrigan C. M., McSween H. Y. Jr., Tenner T. J., Kita N. T., and Bodnar R. J. (2016). CV and CM chondrite impact melts. Geochimica et Cosmochimica Acta 189:228-358.

375. MacDougall, J.D., Lugmair, G.W., Kerridge, J.F. (1984) Early solar system aqueous activity - Sr isotope evidence from the Orgueil Cl meteorite. Nature 307, 249251.

376. Macke R., Consolmagno G., and Britt D. (2011). Density, porosity, and magnetic susceptibility of carbonaceous chondrites. Meteoritics \& Planetary Science, 46, 18421862.

377. Macke R., Consolmagno G., Britt D. and Hutson M. L. (2010). Enstatite chondrite density, magnetic susceptibility, and porosity. Meteoritics \& Planetary Science, 45, 15131526.

378. Mackinnon I. D. R. (1982) Ordered mixed-layer structures on the Mighei carbonaceous chondrites. Geochimica et Cosmochimica Acta 46, 479-489.

379. MacPherson G. J. (2014). Calcium-Aluminum-Rich Inclusions in Chondritic Meteorites. Meteorites and Cosmochemical Processes, Volume 1 of Treatise on Geochemistry (Second Edition). Elsevier, 2014. Edited by Andrew M. Davis, p.139-179.

380. MacPherson G. J. (2005) Calcium-aluminum-rich inclusions in chondritic meteorites. Meteorites, Comets and Planets: Treatise on Geochemistry, Volume 1.(ed. Davis A. M.), pp. 201-246. Elsevier, Amsterdam.

381. MacPherson G. J., Bullock E. S., Janney P. E., Kita N. T., Ushikubo T., Davis A. M., Wadhwa M.; and Krot A. N. (2010). Early Solar Nebula Condensates with Canonical, Not Supracanonical, Initial ${ }^{26} \mathrm{Al} /{ }^{27} \mathrm{Al}$ Ratios. The Astrophysical Journal Letters, 711, L117L121.

382. MacPherson G. J. and Davis A. M. (1994) Refractory inclusions in the prototypical CM chondrite, Mighei. Geochimica et Cosmochimica Acta 58, 5599-5625.

383. MacPherson G. J., Davis A. M. and Zinner E. K. (1995) ${ }^{26} \mathrm{Al}$ in the early solar system: A reappraisal. Meteoritics 30, 365-386.

384. MacPherson G. J. and Delaney J. S. (1985) A fassaite-two-olivine-pleonastebearing refractory inclusion from Karoonda. 16th Lunar and Planetary Science Conference, pp. 515-516 (abstract).

385. MacPherson G. J. and Grossman L. (1984) "Fluffy" Type A Ca-, Al-rich inclusions in the Allende meteorite. Geochimica et Cosmochimica Acta 48, 29-46.

386. MacPherson G. J. and Krot A. N. (2014) The formation of Ca-, Fe-rich silicates in reduced and oxidized CV chondrites: The roles of impact-modified porosity and permeability, and heterogeneous distribution of water ices. Meteoritics \& Planetary Science 49, 1250-1270.

387. MacPherson G. J., Mittlefehldt D. W., Lipschutz M. E., Clayton R. N., Bullock E. S., Ivanov A. V., Mayeda T. K. and Wang M.-S. (2009) The Kaidun chondrite breccias: Petrology, oxygen isotopes, and trace element abundances. Geochimica et Cosmochimica Acta 73, 5493-5511. 
388. MacPherson G., Simon S., Davis A., Grossman L. and Krot A. (2005) Calciumaluminum-rich inclusions: Major unanswered questions. Chondrites and the Protoplanetary Disk, (ed. Krot A. N., Scott E. R. D. and Reipurth B.) pp. 225-250. Astronomical Society of the Pacific, San Francisco.

389. MacPherson G. J., Wark D. A. and Armstrong J. T. (1988) Primitive material surviving in chondrites: Refractory inclusions in: Meteorites and the Early Solar System. (ed. Kerridge J. F. and Matthews M. S.), pp. 746-807, Univ. Arizona, Tucson.

390. Makide K., Nagashima K., Krot A. N., Huss G. R., Hutcheon I. D. and Bischoff A. (2009) Oxygen- and magnesium-isotope compositions of calcium-aluminum-rich inclusions from CR2 carbonaceous chondrites. Geochimica et Cosmochimica Acta 73, 5018- 5050.

391. Marchi S., Delbo' M., Morbidelli A., Paolicchi P., and Lazzarin M. (2009) Heating of Near-Earth Objects and Meteoroids due to close approaches to the Sun. Monthly Notices of the Royal Astronomical Society 400, 147-153.

392. Mason B. and Martin P. M. (1977) Geochemical differences among components of the Allende meteorite. Smithson. Contrib. Earth Sci. 19, 84-95.

393. Matsumoto T., Tsuchiyama A., Gucsik A., Noguchi R., Matsuno J., Nagano T., Imai Y., Shimada A., Uesugi M., Uesugi K., Nakano T., Takeuchi A., Suzuki Y., Nakamura T., Noguchi T., Mukai T., Abe M., Yada T. and Fujimura A. (2012) Micro-Structures of Particle Surfaces of Itokawa Regolith and LL Chondrite Fragments. Lunar and Planetary Science XLIII, Abstract \#1969.

394. Matsumoto, T., Tsuchiyama, T., Miyake, A., Noguchi, T., Nakamura, M., Uesugi, K., Takeuchi, A., Suzuki, Y., and Nakano, T. (2015) Surface and internal structures of a space-weathered rim of an Itokawa regolith particle. Icarus 257, 230-238.

395. Matza S. D. and Lipschutz M. E. (1978) Thermal metamorphism of primitive meteorites-VII. Mineralogy-petrology of heated Murchison (C2) and alteration of C30 and other chondrites. Geochimica et Cosmochimica Acta 42, 1655-1667.

396. Mazanek D. D., Reeves D. M., Abell P. A., Asphaug E., Abreu, N. M., Bell, J. F., Bottke, W. F., Britt, D.T., Campins, H., Chodas, P.W., Ernst, C. M., Fries, M. D., Gertsch, L. S., Glavin, D. P., Hartzell, C.M., Hendrix, A. R., Nuth, J. A., Scheeres, D. J., Sercel, J. C., Takir, D., and Zacny, K. (2016) Asteroid Redirect Mission (ARM) Formulation Assessment and Support Team (FAST) Final Report. NASA Technical Memorandum TM2016-210911, $130 \mathrm{pp}$.

397. Mazrouei, S., Daly, M. G., Barnouin, O. S., Ernst, C. M., and DeSouza, I. (2014) Block distributions on Itokawa. Icarus 229, 181-189.

398. McAdam M. M., Sunshine J. M., Howard K. T., and McCoy T. M. (2015) Aqueous alteration on asteroids: Linking the mineralogy and spectroscopy of $\mathrm{CM}$ and $\mathrm{Cl}$ chondrites. Icarus 245, 320-332.

399. McAdam M. M., Sunshine J. M., Howard K. T., Alexander C. M., McCoy T. J., and Bus, S. J. (2018). Spectral evidence for amorphous silicates in least-processed CO meteorites and their parent bodies. Icarus, 306, 32-49.

400. McGuire A. V. and Hashimoto A. (1989) Origin of zoned fine- grained inclusions in the Allende meteorite. Geochimica et Cosmochimica Acta 53, 1123-1133.

401. McKeegan K. D. and Davis, A. M. (2003). Early Solar System Chronology. Treatise on Geochemistry, Volume 1. Editor: Andrew M. Davis. Executive Editors: Heinrich D. Holland and Karl K. Turekian. pp. 711. ISBN 0-08-043751-6. Elsevier, 2003., p.431-460.

402. McKeegan K. D., Kallio A. P. A., Heber V. S., Jarzebinski G., Mao P. H., Coath C. D., Kunihiro T., Wiens R. C., Nordholt J. E., Moses R. W., Reisenfeld D. B., Jurewicz A. 
J. G. and Burnett D. S. (2011) The oxygen isotopic composition of the Sun inferred from captured solar wind. Science 332, 1528-1532.

403. McNamara D. D. (2012). Omphacite-a mineral under pressure! Geol Today $28,71-75$.

404. McSween H. Y. (1977a) Petrographic variations among carbonaceous chondrites

of the Vigarano type. Geochimica et Cosmochimica Acta 41, 1777-1790.

405. McSween H. Y. (1977b) Carbonaceous chondrites of the Ornans type: A metamorphic sequence. Geochimica et Cosmochimica Acta 41, 477-491.

406. McSween H. Y., Jr. (1979a) Are carbonaceous chondrites primitive or processed?

A review. Reviews of Geophysica and Space Physics 17, 1059-1078.

407. McSween H. Y., Jr. (1979b) Alteration in CM carbonaceous chondrites inferred from modal and chemical variations in matrix. Geochimica et Cosmochimica Acta 43, 1761-1770.

408. McSween H. Y., Jr. (1987) Aqueous alteration in carbonaceous chondrites: Mass balance constraints on matrix mineralogy. Geochimica et Cosmochimica Acta 51, 24692477.

409. McSween H.Y. Jr. (1999). Meteorites and their parent planets, 2nd ed. Cambridge: Cambridge University Press. 310 p.

410. McSween H. Y., Ghosh A., Grimm R. E., Wilson L. and Young E. D. (2002) Thermal evolution models of asteroids. In Asteroids III (ed. Bottke W. F., Cellino A., Paolicchi P. and Binzel R. P.), pp. 559-571. Univ. Arizona Press, Tucson.

411. McSween H. Y., Jr. and Richardson, S. M. (1977) The composition of carbonaceous chondrite matrix. Geochimica et Cosmochimica Acta 41, 1145-1161.

412. Metzler K. (1995) Aqueous alteration of primary rock on the CM parent body. Lunar Planet. Sci. XXVI, p. 961.

413. Michel, P. and Delbo, M. (2010) Orbital and thermal evolutions of four potential targets for a sample return space mission to a primitive near-Earth asteroid. Icarus 209, 520-534.

414. Michel, P., Morbidelli, A. and Bottke, W.F. (2005) Origin and dynamics of nearEarth objects. C. R. Phys. 6, 291-301.

415. Michel P. and Richardson D. C. (2013) Collision and gravitational reaccumulation: Possible formation mechanism of the asteroid Itokawa. Astronomy and Astrophysics 554, L1-L4.

416. Michel-Lévy M. C (1969). Étude minéralogique de la chondrite C III de Lancé. Comparaison avec d'autres météorites du même groupe. Meteorite Research, Proceedings of a Symposium held in Vienna, Austria, August 7-13, 1968, Dordrecht: Reidel, 1969, edited by Peter M. Millman. Astrophysics and Space Science Library, Vol. 12, p.492.

417. Michikami, T., Hagermann, A., Kadokawa, T., Yoshida, A., Shimada, A., Hasegawa, S., and Tsuchiyama, A. (2016) Fragment shapes in impact experiments ranging from cratering to catastrophic disruption. Icarus 264, 316-330.

418. Michikami, T., Nakamura, A. M., and Hirata, N. (2010) The shape and distribution of boulders on Asteroid 25143 Itokawa: Comparison with fragments from impact experiments. Icarus 207, 277-284.

419. Michikami T., Nakamura A. M., Hirata N., et al., (2008) Size-frequency statistics of boulders on global surface on asteroid 25143 Itokawa. Earth Planets Space 60, 13-20.

420. Midgley, H.G., Gross, K.A., 1956. Thermal reactions of smectites. Clay Mineral Bulletin 3, 79-90. 
421. Miura, Y.N., Noguchi, T., Tsuchiyama, A., Yano, H., Yoshida, S., and Nagata, K., (2008). Compressive strength measurements of meteorites and terrestrial rocks: implications for physical properties of asteroidal surfaces (abstract). Proceedings of Japan Geoscience Union Meeting (P168-P002).

422. Miyamoto H., Yano H., Scheeres D., et al., (2007) Regolith migration and sorting on asteroid Itokawa. Science 316, 1011-1014.

423. Moghadam R. H., Trepmann C. A., Stockhert B., and Renner J. (2010) Rheology of synthetic omphacite aggregates at high pressure and high temperature. Journal of Petrology 51, 921-945.

424. Morbidelli, A., Bottke, W.F., Froeschlé, Ch. and Michel, P. (2002) Origin and evolution of near-Earth objects. In: Bottke, W.F., Cellino, A., Paolicchi, P., Binzel, R.P. (Eds.), Asteroids III. University of Arizona Press, Tucson, pp. 409-422.

425. Morlok A., Bischoff A., Stephan T., Floss C., Zinner E. and Jessberger E. K. (2006) Brecciation and chemical heterogeneities of $\mathrm{Cl}$ chondrites. Geochimica et Cosmochimica Acta 70, 5371-5394.

426. Morlok A. and Libourel G. (2013). Aqueous alteration in CR chondrites: Meteorite parent body processes as analogue for long-term corrosion processes relevant for nuclear waste disposal. Geochimica et Cosmochimica Acta 103, 76-103.

427. Moskovitz N. (2012). Colors of dynamically associated asteroid pairs. Icarus 221, 63-71.

428. Mothé-Diniz T., Carvano J. M., Bus S. J., Duffard R. and Burbine T. H. (2008) Mineralogical analysis of the Eos family from near-infrared spectra. Icarus 195, 277-294.

429. Moyano-Cambero C., Trigo-Rodríguez J., Llorca J., Fornasier S., Barucci M., Rimola A. (2016) A plausible link between the asteroid 21 Lutetia and $\mathrm{CH}$ carbonaceous chondrites. Meteoritics \& Planetary Sciences 51, 1795-1812.

430. Müller T. G., O’Rourke L., Barucci A. M., Pál A., Kiss C., Zeidler P., Altieri B., González-García B. M. and Küppers M. (2012) Physical properties of OSIRIS-REx target asteroid (101955) 199 RQ36. Astronomy and Astrophysics 548, A36.

431. Müller W., Kurat G., Kracher A. (1979) Chemical and crystallographic study of cronstedtite in the matrix of the Cochabamba (CM2) carbonaceous chondrite. Tschermaks Mineral. Petrogr. Mitt. 26, 293-304.

432. Murdoch N., Sánchez P., Schwartz S R., et al., (2015), in Michel P., DeMeo F. E., Bottke W. F., eds, Asteroids IV . Univ. Arizona Press, Tucson, p. 767.

433. Nagashima K., Krot, A. N., and Huss G. R. (2014). ${ }^{26} \mathrm{Al}$ in chondrules from CR2 chondrites. Geochemical Journal, 48, 561-570.

434. Nakamura E., Makishima A., Moriguti T., Kobayashi K., Tanaka R., Kunihiro T., Tsujimori T., Sakaguchi C., Kitagawa H., Ota T., Yachi Y., Yada T., Abe M., Fujimura A., Ueno M., Mukai T., Yoshikawa M. and Kawaguchi J. (2012) Space environment of an asteroid preserved on micrograins returned by the Hayabusa spacecraft. Proc. Nat. Acad. Sci. 109, E624-E629.

435. Nakamura K., Zolensky M., Tomita S., Nakashima S. and Tomeoka K. (2002) Hollow organic globules in the Tagish Lake meteorite as possible products of primitive organic reactions. International Journal of Astrobiology 1, 179-189.

436. Nakamura T., Noguchi T., Tanaka M., Zolensky M. E., Kimura M., Tsuchiyama A., Nakato A., Ogami T., Ishida H., Uesugi M., Yada T., Shirai K., Fujimura A., Okazaki R., Sandford S. A., Ishibashi Y., Abe M., Okada T., Ueno M., Mukai T., Yoshikawa M. and Kawaguchi J. (2011) Itokawa dust particles: A direct link between S-type asteroids and ordinary chondrites. Science 333, 1113-1116. 
437. Nakamuta Y., Nakamura T., and Nakamura N. (2001) Metamorphic temperature of Kobe meteorite estimated by the plagioclase thermometer. Antarctic Meteorites 26, 98100 (abstract).

438. Nakamuta Y., Nakamura T., and Nakamura N. (2006) Structural state of plagioclase from the Kobe CK chondrite: Implications for the thermal history of the CK parent body. Journal of Mineralogy and Petrology Sciences 101, 308-318.

439. Nakamura-Messenger K., Messenger S., Keller L.P., Clemett S.J., and Zolensky M.E. (2006) Organic Globules in the Tagish Lake Meteorite: Remnants of the Protosolar Disk. Science 314, 1439-1442.

440. Neff K. E. and Righter K. (2006) Opaque assemblages in CK and CV carbonaceous chondrites. 37th Lunar and planetary Science Conference, \#1320 (abstract).

441. Nesvorný D., Vokrouhlický D., Morbidelli A., and Bottke W. F. (2009) Asteroidal source of $L$ chondrite meteorites. Icarus 200, 698-701.

442. Nishiizumi, K., and Caffee, M.W. (2009) Exposure history of CM2 carbonaceous chondrites - update. Abstract, 72nd Meteoritical Society Annual Meeting, Meteoritics \& Planetary Science 44, A158.

443. Nishiizumi K. and Caffee M.W. (2012) LPSC XLIII, abst \#2758.

444. Nittler L. R., Alexander C. M. O'D., adn Stroud, R. M. (2013). High Abundance of Presolar Materials in CO3 Chondrite Dominion Range 08006. LPI Contribution No. 1719, p.2367.

445. Noguchi T. (1993) Petrology and mineralogy of CK chondrites: Implications for the metamorphism of the CK chondrite parent body. Proc. NIPR Symp. Antarct. Met. 6, 204233.

446. Noguchi, T., Tsuchiyama, A., Hirata, N., Demura, H., Nakamura, R., Miyamoto, H., Yano, H., Nakamura, T., Saito, J., Sasaki, S., Hashimoto, T., Kubota, T., Ishiguro, M., and Zolensky. M.E. (2010) Surface morphological features of boulders on Asteroid 25143 Itokawa. Icarus 206, 319-326.

447. Noguchi T., Nakamura T., Kimura M., Zolensky M. E., Tanaka M., Hashimoto T., Konno M., Nakato A., Ogami T., Fujimura A., Abe M., Yada T., Mukai T., Ueno M., Okada T., Shirai K., Ishibashi Y. and Okazaki R. (2011) Incipient space weathering observed on the surface of Itokawa dust particles. Science 333, 1121-1125.

448. Noguchi, T., Kimura, M., Hashimoto, T., Konno, M., Nakamura, T., Nakato, A., Ogami, T., Ishida, H., Sagae, R., Tsujimoto, S., Tsuchiyama, A., Zolensky, M.E., Tanaka, M., Fujimura, A., Abe, M., Yada, T., Mukai, T., Ueno, M., Okada, T., Shirai, K., Ishibashi, Y., Okazaki, R. (2012) Space Weathering Products Found on the Surfaces of the Itokawa Dust Particles: A Summary of the Initial Analysis. Lunar and Planetary Science XLIII, Abstract \#1896.

449. Noguchi, T., Kimura, M., Hashimoto, T., Konno, M., Nakamura, T., Zolensky, M.E., Okazaki, R., Tanaka, M., Tsuchiyama, A., Nakato, A., Ogami, T., Ishida, H., Sagae, R., Tsujimoto, S., Matsumoto, S., Matsuno, J., Fujimura, A., Abe, M., Yada, T., Mukai, T., Ueno, M., Okada, T., Shirai, K., and Ishibashi, Y. (2014) Space weathered rims found on the surfaces of the Itokawa dust particles. Met. Planet. Sci. (Hayabusa Special Issue) 49, 188-214.

450. Nomura K. and Miyamoto M. (1998) Hydrothermal experiments on alteration of Ca-Al-rich inclusions (CAls) in carbonaceous chondrites: Implication for aqueous alteration in parent asteroids. Geochimica et Cosmochimica Acta 62, 3575-3588. 
451. Noronha B. A. and Friedrich J. M. (2014) Chemical compositions and classification of five thermally altered carbonaceous chondrites. Meteoritics and Planetary Science 49, 1494-1504.

452. Oberti R. and Caporuscio F. A. (1991). Crystal-chemistry of clinopyroxenes from mantle eclogites-a study of the key role of the M2 site population by means of crystalstructure refinement. American Mineralogist 76, 1141-1152

453. Ogliore, R.C., and Dobrică, E. (2015) Space-weathering features on two Hayabusa particles. Lunar and Planetary Science XLVI, Abstract \#1631.

454. Ohnishi I. and Tomeoka K. (2007) Hydrothermal alteration experiments of enstatite: Implications for aqueous alteration of carbonaceous chondrites. Meteoritics \& Planetary Science 42, 49-61.

455. Ohnishi K., Tomeoka K., and Ishizaki N. (2007) Microinclusion-rich vesicular olivine in the Karoonda CK4 chondrite: transmission electron microscopy. Journal of Mineralogy and Petrological Sciences 102, 346-351.

456. Okada, T., Shirai, K., Yamamoto, Y., Arai, T., Ogawa, K., Hosono, K., and Kato, M. (2006) X-ray Fluorescence Spectrometry of Asteroid Itokawa by Hayabusa. Science, 312, 1338-1341.

457. Olsen M. B., Wielandt D., Schiller M., Van Kooten E. M. M. E., and Bizzarro, M. (2016). Magnesium and ${ }^{54} \mathrm{Cr}$ isotope compositions of carbonaceous chondrite chondrules - Insights into early disk processes. Geochimica et Cosmochimica Acta, 191, 118-138.

458. Opeil C., Colsolmagno G., Safarik D., Britt D. (2012) Stony meteorite thermal properties and their relationship with meteorite chemical and physical states. Meteoritics \& Planetary Science, 47, 319-329.

459. Palme H., Jones R. (2003) Solar system abundances of the elements. In Treatise on Geochemistry. Heinrich D. Holland and Karl K. Turekian Eds., Elsevier Science., p 541-580.

460. Pätzold M., Andert T., Asmar S., Anderson J., Barriot J.-P., Bird M., Häusler B., et al. (2011). Asteroid 21 Lutetia: Low mass, high density. Science 334, 491-2.

461. Pearson V. K., Sephton M. A., Franchi I. A., Gibson, J. M., and Gilmour I. (2006). Carbon and nitrogen in carbonaceous chondrites: Elemental abundances and stable isotopic compositions. Meteoritics \& Planetary Science, 41, 1899-1918.

462. Petaev M. I. and Jacobsen S. B. (2009) Petrologic study of SJ101, a few forsteritebearing CAl from the Allende CV3 chondrite. Geochimica et Cosmochimica Acta 73, 51005114.

463. Petaev M. I., Meibom A., Krot A. N., Wood J. A., and Keil K. (2001) The condensation origin of zoned metal grains in Queen Alexandra Range 94411: Implications for the formation of the Bencubbin-like chondrites. Meteoritics \& Planetary Science 36, 93-106.

464. Petaev M. I. and Wood J. A. (1998) The consensation with partial isolation (CWPI) model of condensation in the solar nebula. Meteoritics \& Planetary Science. 33, 11231137.

465. Pignatelli I., Marrocchi Y., Vacher L.G., Delon R., and Gounelle M. (2016) Multiple precursors of secondary mineralogical assemblages in CM chondrites. Meteorit. Planet. Sci. 51, 785-805.

466. Pignatelli I., Marrocchi Y., Muglaioli E., Bourdelle F., and Gounelle M. (2017) Mineralogical, crystallographic and redox features of the earliest stages of fluid alteration in CM chondrites. Geochim. Cosmochim. Acta 209, 106-122. 
467. Pizzarello S., Cooper G., Flynn G. (2005) The nature and distribution of the organic material in carbonaceous chondrites and interplanetary dust particles. In Meteorites and the Early Solar System II, D. Lauretta and H. McSween, Eds., Univ. Arizona Press. p. 625-651.

468. Pizzarello S., Huang Y., Becker L., Poreda R. J., Nieman R. A., Cooper G., and Williams M. 2001. The organic content of the Tagish Lake meteorite. Science 293:22362239.

469. Pizzarello S., Schrader D. L., Monroe A. A., and Lauretta D. S. (2012). The chiral composition of primitive CR meteorites and the diverse effects of water in cosmochemical evolution. Proceedings of the National Academy Science USA 109:11949-11954.

470. Powers M. C. (1953) A new roundness scale for sedimentary particles. Journal of Sedimentary Petrology 23, 117-119.

471. Pratesi G., Salvadori A., Moggi-Cecchi V., Franchi I., and Greenwood R. C. 2006. A new CK carbonaceous chondrite from Hammada Al Hamra, Libya. 37th Lunar and Planetary Science Conference, \#1899 (abstract).

472. Prinz M., Weisberg M. K., and Nehru C. E. (1988) Gunlock, a new Type 3 ordinary chondrite with a golfball-sized chondrule. Meteoritics 23, 297.

473. Qin L., Alexander C. M. O’D., Carlson R. W., Horan M. F., Yokoyama T. (2010) Contributors to chromium isotope variation of meteorites. Geochimica et Cosmochimica Acta 74, 1122-1145.

474. Ralchenko M., Britt D. T., Samson C., Herd C. D. K., Herd R. K., and McCausland P. J. A. (2014). Bulk physical properties of the Tagish Lake meteorite frozen pristine fragments (abstract). LPI Contribution No. 1777, p.1021.

475. Reddy, V., LeCorre, L., Hicks, M., Lawrence, K., Buratti, B.J., Abell, P.A., Gaffey, M.J., and Hardersen, P.S. (2012) Composition of near-Earth Asteroid 2008 EV5: Potential target for robotic and human exploration. Icarus 221, 678-681.

476. Richardson, S.M. (1978) Vein formation in the $\mathrm{Cl}$ carbonaceous chondrites. Meteoritics 13, 141-159.

477. Riciputi, L.R., McSween Jr., H.Y., Johnson, C.A., Prinz, M. (1994) Minor and trace element concentrations in carbonates of carbonaceous chondrites, and implications for compositions of co-existing fluids. Geochim. Cosmochim. Acta 58 (4), 1343-1351.

478. Righter K. and Neff K. E. (2007) Temperature and oxygen fugacity constraints on $\mathrm{CK}$ and $\mathrm{R}$ chondrites and implications for water and oxidation in the early solar system. Polar Science 1, 25-44.

479. Riley, N.A. (1941) Projection sphericity. Journal of Sedimentary Petrology 11, 9497.

480. Rivkin A., Howell E., Vilas F., Lebofsky L. (2002) Hydrated Minerals on Asteroids: The Astronomical Record. Asteroids III, U of A Press, Bottke W., Cellino A., Paolo Paolicchi P., Richard P. Binzel R., Eds., p. 235-253.

481. Robinson M. S., Thomas P. C., Veverka J., et al. (2001) The nature of ponded deposits on Eros. Nature 413 (6854), 396-400.

482. Robinson M. S., Thomas P. C., Veverka J., et al. (2002) The geology of 433 Eros. Meteoritics \& Planetary Science 37, 1651-1684.

483. Rochette P., Gattacceca J., Bonal L., Bourot-Denise M., Chevrier M., Clerc J.-P., Consolmagno G., Folco L., Gounelle M., Kohout T., Pesonen L., Quirico E., Sagnotti L., and Skripnik A. (2008). Magnetic classification of stony meteorites: 2. Non-ordinary chondrites. Meteoritics \& Planetary Science 43:959-980. 
484. Rubin A. E. (1984) Coarse-grained chondrule rims in type 3 chondrites. Geochimica et Cosmochimica Acta 48, 1779-1789.

485. Rubin A. E. (1989). Size frequency distributions of chondrules in CO3 chondrites. Meteoritics, 24, 179-189.

486. Rubin A. E. (1991) Euhedral awaruite in the Allende meteorite: Implications for the origin of awaruite- and magnetite-bearing nodules in CV3 chondrites. American Mineralogist 76, 1356-1362.

487. Rubin A. E. (1991) Silicate darkening and heterogeneous plagioclase in CK and ordinary chondrites. Meteoritics 26, 339 (abstract).

488. Rubin A. E. (1992) A shock-metamorphic model for silicate darkening and compositionally variable plagioclase in $\mathrm{CK}$ and ordinary chondrites. Geochimica et Cosmochimica Acta 56, 1705-1714.

489. Rubin, A. E. (1993) Magnetite-sulfide chondrules and nodules in CK carbonaceous chondrites : Implications for the timing of CK oxidation, Meteoritics 28, 130-I 35.

490. Rubin A. E. (1995) Petrologic evidence for collisional heating of chondritic asteroids. Icarus 113, 156-167.

491. Rubin A. E. (1997) Mineralogy of meteorite groups. Meteoritics \& Planetary Science 32, 231-247.

492. Rubin A. E. (1998). Correlated petrologic and geochemical characteristics of CO3 chondrites. Meteoritics \& Planetary Science, 33, 383-391.

493. Rubin, A. E. (2003). Chromite-plagioclase assemblages as a new shock indicator; implications for the shock and thermal histories of ordinary chondrites. Geochimica et Cosmochimica Acta 67, 2695-2709.

494. Rubin A. E. (2004) Post-shock annealing and post-annealing shock in equilibrated ordinary chondrites: implications for the thermal and shock histories of chondritic asteroids. Geochim. Cosmochim. Acta 68, 673-689.

495. Rubin A. E. (2010) Physical properties of chondrules in different chondrite groups: Implications for multiple melting events in dusty environments. Geochimica et Cosmochimica Acta 74, 4807-4828.

496. Rubin A. E. (2011) Origin of the differences in refractory-lithophile-element abundances among chondrite groups. Icarus 213, 547-558.

497. Rubin A. E. (2012a) A new model for the origin of Type-B and Fluffy Type-A CAls: Analogies to remelted compound chondrules. Meteoritics \& Planetary Science 47, 10621074.

498. Rubin A. E. (2012b) Collisional facilitation of aqueous alteration of CM and CV carbonaceous chondrites. Geochimica et Cosmochimica Acta 90, 181-194.

499. Rubin A. E. (2013) An amoeboid olivine inclusion (AOI) in CK3 NWA 1559, comparison to AOIs in CV3 Allende, and the origin of AOls in CK and CV chondrites. Meteoritics \& Planetary Science 48, 432-444.

500. Rubin A. E. (2015) An American on Paris: Extent of aqueous alteration of a CM chondrite and the petrography of its refractory and amoeboid olivine inclusions. Meteoritics and Planetary Science 50, 1595-1612.

501. Rubin A. E. and Huber H. (2005) A weathering index for CK and R chondrites. Meteoritics \& Planetary Science 40, 1123-1130.

502. Rubin A. E., James J. A., Keck B. D., Weeks K. S, Sears D. W. G., and Jarosewich E. (1985). The Colony meteorite and variations in CO3 chondrite properties. Meteoritics 20:175-196. 
503. Rubin A. E. and Ma C. (2017) Meteoritic minerals and their origins. Chemie der Erde 77, 325-385.

504. Rubin A. E., Trigo-Rodríguez J. M., Huber H. and Wasson J. T. (2007) Progressive alteration of CM carbonaceous chondrites. Geochimica et Cosmochimica Acta 71, 23612382.

505. Rubin A. E., Wang D., Kallemeyn G. W., and Wasson J. T. (1988) The Ningqiang meteorite: Classification and petrology of an anomalous CV chondrite. Meteoritics 23, 1323.

506. Rubin A. E. and Wasson J. T. (1987) Chondrules, matrix and coarse-grained chondrule rims in the Allende meteorite: Origin, interrelationships and possible precursor components. Geochimica et Cosmochimica Acta 51, 1923-1937.

507. Russell S. S., Huss G. R., Fahey A. J., Greenwood R. C., Hutchison R., and Wasserburg G. J. (1998) An isotopic and petrologic study of calcium-aluminum-rich inclusions from CO3 meteorites. Geochimica et Cosmochimica Acta 62:689-714.

508. Russell S. S., Krot A. N., Huss G. R., Keil K., Itoh S., Yurimoto H. and MacPherson G. J. (2005) The genetic relationship between refractory inclusions and chondrules. In Chondrules and the Protoplanetary Disk (ed. Krot A. N., Scott E. R. D. and Reipurth B.), pp. 317-350. Astronomical Society of the Pacifric, San Francisco.

509. Ruzicka, A. (2012) Chondrule formation by repeated evaporative melting and condensation in collisional debris clouds around planetesimals. Meteoritics \& Planet. Sci., 47, 2218-2236.

510. Saito J., Miyamoto H., Nakamura R., et al. (2006) Detailed images of asteroid 25143 Itokawa from Hayabusa. Science 312, 1341-1344.

511. Sanders N. E. and Velbel M. A. (2012) The size distributions of nanoscale Fe-Ni$S$ droplets in Stardust melted grains from comet 81P/Wild 2. Meteoritics \& Planetary Science 47, 594-612.

512. Scheeres D. J. (2005) Solar radiation pressure and transient flows on asteroid surfaces. Lunar Planet. Sci. XXXVI, Abstract \#1919. Lunar and Planetary Institute, Houston.

513. Scherer P. and Schultz L. (2000) Noble gas record, collisional history, and pairing of CV, CO, CK, and other carbonaceous chondrites. Meteoritics \& Planetary Science 35, 145-153.

514. Schrader D. L., Connolly H. C. Jr., and Lauretta D. S. (2008). Opaque phases in type-Il chondrules from CR2 chondrites: Implications for CR parent body formation. Geochimica et Cosmochimica Acta 72:6124-6140.

515. Schrader D. L., Connolly H. C. Jr., Lauretta D. S., Nagashima K., Huss G. R., Davidson J., and Domanik K. J. (2013). The formation and alteration of the Renazzo-like carbonaceous chondrites II: Linking O-isotope composition and oxidation state of chondrule olivine. Geochimica et Cosmochimica Acta 101:302-327.

516. Schrader D. L., Connolly, Jr., H. C., Lauretta D. S., Zega T. J., Davidson J. and Domanik K. J. (2015) The formation and alteration of the Renazzo-like carbonaceous chondrites III: toward understanding the genesis of ferromagnesian chondrules. Meteoritics \& Planetary Science 50, 15-50.

517. Schrader D. L., Franchi I. A., Connolly H. C. Jr., Greenwood R. C., Lauretta D. S., and Gibson J. M. (2011). The formation and alteration of the Renazzo-like carbonaceous chondrites I: Implications of bulk-oxygen isotopic composition. Geochimica et Cosmochimica Acta 75:308-325. 
518. Schrader D. L., Nagashima K., Krot A. N., Ogliore R. C., and Hellebrand E. (2014). Variations in the $\mathrm{O}$-isotope composition of gas during the formation of chondrules from the CR chondrites. Geochimica et Cosmochimica Acta 132:50-74.

519. $\quad$ Schrader D. L., Nagashima K., Krot A. N., Ogliore R. C., Yin Q.- Z., Amelin Y. A., Stirling C. H. and Kaltenbach A. (2017) Distribution of ${ }^{26} \mathrm{Al}$ in the CR chondrite chondruleforming region of the protoplanetary disk. Geochimica et Cosmochimica Acta, 201, 275302.

520. Schrader D. L., Nagashima K., Waitukaitis S. R., Davidson J., McCoy T. J., Connolly H. C., and Lauretta D. S. (2018). The retention of dust in protoplanetary disks: Evidence from agglomeratic olivine chondrules from the outer Solar System. Geochimica et Cosmochimica Acta, 223, 405-421.

521. Scott E. R. D., Keil K., and Stoeffler D. (1992) Shock metamorphism of carbonaceous chondrites. Geochimica and Cosmochimica Acta 56, 4281-4293.

522. Scott E. R. D. and Krot A. N. (2005) Chondrites and their components. In Meteorites, Comets and Planets, (ed. Davis A. M.), pp. 143-200, Elsevier, Amsterdam.

523. Scott E. R. D., Love S. G., and Krot A. N. (1996) Formation of chondrules and chondrites in the protoplanetary nebula. In Chondrules and the protoplanetary disk, edited by Hewins R. H., Jones R. H., and Scott E. R. D. Cambridge, UK: Cambridge University Press. pp. 87-96.

524. Scott E. R. D. and Jones R. H. (1990). Disentangling nebular and asteroidal features of $\mathrm{CO} 3$ carbonaceous chondrites. Geochimica et Cosmochimica Acta, 54, 24852502.

525. Scott E. R. D., Rubin A. E., Taylor G. J. and Keil K. (1984). Matrix material in type 3 chondrites -- Occurrence, heterogeneity and relationship with chondrules. Geochimica et Cosmochimica Acta 48, 1741-1757.

526. Scott E. R. D. and Taylor G. J. (1985). Petrology of types 4-6 carbonaceous chondrites. Porceedings of the 15th Lunar and Planetary Science Conference, C699C709.

527. Sears D. W. G., Batchelor J. D., Lu Jie, and Keck, B. D. (1991). Metamorphism of $\mathrm{CO}$ and CO-like chondrites and comparisons with type 3 ordinary chondrites. 15th Symposium on Antarctic Meteorites. Proceedings of the NIPR Symposium, No. 4. Published by the National Institute of Polar Research, 319.

528. Sears D. W. G., Grossman J. N., Melcher C. L., Ross L. M., and Mills A. A. (1980). Measuring metamorphic history of unequilibrated ordinary chondrites. Nature 287, 791795.

529. Sears D. W. G., Morse A. D., Hutchison R., Guimon R. K., Jie Lu, Alexander C. M. O'd., Benoit P. H., Wright I., Pillinger C., Xie Tian, and Lipschutz M. E. (1995). Metamorphism and aqueous alteration in low petrographic type ordinary chondrites. Meteoritics 30, 169-181.

530. Sekigawa C. and Keller L.P. (2010) The Mineralogy, Petrography and Chemistry of Comet Dust: Sulfides. LPSC XLI, \#2405.

531. Senshu H., Kimura H., Yamamoto T., Wada K., Kobayashi M., Namiki N., Matsui T. (2015) Photoelectric dust levitation around airless bodies revised using realistic photoelectron velocity distributions. Planetary and Space Science 116, 18-29.

532. Shinbrot T., Sabuwala T., Siu T., et al., (2017) Size Sorting on the Rubble-Pile Asteroid Itokawa. Physical Review Letters 118, 111101 
533. Shu F. H., Shang H., Gounelle M., Glassgold A. E. and Lee T. (2001) The origin of chondrules and refractory inclusions in chondritic meteorites. Astrophys. J. 548, 10291050.

534. Shukolyukov A. and Lugmair G. W. (2006) Manganese-chromium isotope systematics of carbonaceous chondrites. Earth and Planetary Science Letters 250, 200213.

535. Simon S. B. and Haggerty S. E. (1979) Petrography and olivine mineral chemistry of chondrules and inclusions in the Allende meteorite. Proc. Lunar Planet. Sci. Conf. 10th, 871-883.

536. Simon S. B., Grossman L., Casanova I., Symes S., Benoit P., Sears D. W. G. and Wacker J. F. (1995) Axtell, a new CV3 chondrite from Texas. Meteoritics 30, 42-46.

537. Simon S. B., Davis A. M. and Grossman L. (1999) Origin of compact type A refractory inclusions from CV3 carbonaceous chondrites. Geochimica et Cosmochimica Acta 63, 1233-1248.

538. Smith C. L. and Russell S. S. (2003). Dar Al Gani 431: Unravelling nebular and parent body processes (abstract). Meteoritics \& Planetary Science 38:A5222.

539. Stephant A., Remusat L., and Robert F. (2017) Water in type 1 chondrules of Paris CM chondrite. Geochimica et Cosmochimica Acta 199, 75-90.

540. Stöffler D., Bischoff A., Buchwald V., and Rubin A. (1988) Shock effects in meteorites. In: Meteorites and the early solar system (J. F. Kerridge, M. S. Matthews, eds.), University of Arizona Press, pp. 165-202.

541. Stöffler D., Keil K., Scott E. R. D. (1991) Shock metamorphism of ordinary chondrites. Geochimica and Cosmochimica Acta 55, 3845-3867.

542. Sugiura N., Petaev M. I., Kimura M., Miyazaki A., and Hiyagon H. (2009). Nebular history of amoeboid olivine aggregates. Meteoritics \& Planetary Science, 44, 559-572.

543. Tachibana Y., Kitamura M., Hirajima T., Nakamura N. (2002) Equilibration temperature of the Kobe meteorite. Geochemical Journal 36, 323-332.

544. Takenouchi, A., Zolensky, M. E., Nishiizumi, K., Caffee, M., Velbel, M. A., Ross, K., Zolensky, A., Le, L., Imae, N., Yamaguchi, A., and Mikouchi, T. (2013). What are space exposure histories telling us about CM carbonaceous chondrites? NIPR Annual Conference on Antarctic Meteorites, Abstracts.

545. Takenouchi, A., Zolensky, M. E., Nishiizumi, K., Caffee, M., Velbel, M. A., Ross, K., Zolensky, P., Le, L., Imae, N., Yamaguchi, A., and Mikouchi, T. (2014). On the relationship between cosmic-ray exposure ages and petrography of CM chondrites. 45th Lunar and Planetary Science Conference, Abstract \#1827.

546. Tancredi G., Roland S., Bruzzone., (2015) Distribution of boulders and the gravity potential on asteroid Itokawa. Icarus 247, 279-290.

547. Tang H., Dauphas N. (2012) Abundance, distribution, and origin of ${ }^{60} \mathrm{Fe}$ in the solar protoplanetary disk. EPSL 359-360, 248-263.

548. Teller, J. T. (1976) Equantcy versus sphericity. Sedimentology 23, 427-428.

549. Tenner T. J., Nakashima D., Ushikubo T., Kita N. T. and Weisberg M. K. (2015) Oxygen isotope ratios of FeO-poor chondrules in CR3 chondrites: Influence of dust enrichment and $\mathrm{H}_{2} \mathrm{O}$ during chondrule formation. Geochimica et Cosmochimica Acta, 148, 228-250.

550. Thomas P. C., Veverka J., Robinson M. S., and Murchie S. (2001) Shoemaker crater as the source of most ejecta blocks on the asteroid 433 Eros. Nature 413, 394-396. 
551. Thompson, M.S., Christoffersen, R., Zega, T.J., and Keller, L.P. (2014) Microchemical and structural evidence for space weathering in soils from asteroid Itokawa. Earth, Planets and Space 66, article \#89.

552. Tomeoka K., Buseck P. (1988) Matrix mineralogy of the Orgueil Cl carbonaceous chondrite. Geochimica et Cosmochimica Acta 52, 1627-1640.

553. Tomeoka K. and Itoh D. (2004). Sodium-metasomatism in chondrules in CO3 chondrites: relationship to parent body thermal metamorphism. Meteoritics \& Planetary Science, 39, 1359-1373.

554. Tomeoka K., Kojima T., Ohnishi I., Ishii Y., and Nakamura N. (2005). The Kobe CK carbonaceous chondrite: Petrography, mineralogy and metamorphism. Journal of Mineralogical and Petrological Sciences 100, 116-125.

555. Tomeoka K., Kojima H., Yanai K. (1989a) Yamato-86720: a CM carbonaceous chondrite having experienced extensive aqueous alteration and thermal metamorphism. Proceeding of NIPR Symp. Antarct. Meteorites 2, 55-74.

556. Tomeoka, K., Kojima, H., Yanai, K. (1989b) Yamato-82162: a new kind of Cl carbonaceous chondrite from Antarctica. Proc. NIPR Symp. Antarct. Meteorites 2, 36-54.

557. Tomeoka K., Nomura K., and Takeda H. (1992). Na-bearing Ca-Al-rich inclusions in the Yamato-791717 CO carbonaceous chondrite. Meteoritics, 27, 136-143.

558. Tomeoka K., Ohnishi I., and Nakamura N. (2001). Silicate darkening in the Kobe CK chondrite: Evidence for shock metamorphism at high temperature. Meteoritics and Planetary Science 36, 1535-1545.

559. Tonui E. K., Zolensky M. E. and Lipschutz M. E. (2002) Petrography, mineralogy and trace element chemistry of Y-86029, LEW-85332 and Y-793321: aqueous alteration and heating events. Antarct. Meteorit. Res. 15, 38-58.

560. Tonui E. K., Zolensky M. E., Lipschutz M. E., Wang M.-S. and Nakamura T. (2003) Yamato-86029: aqueously altered and thermally metamorphosed $\mathrm{Cl}$ chondrite with unusual textures. Meteoritics \& Planetary Science 38, 269-292.

561. Tonui, E. K., Zolensky, M., Hiroi, T., Nakamura, T., Lipschutz, M. E., Wang, M.-S., and Okidura, K. (2014) Petrographic, chemical and spectroscopic evidence for thermal metamorphism in carbonaceous chondrites I. Cl and CM chondrites. Geochimica et Cosmochimica Acta 126, 284-306.

562. Travis B. J. and Schubert G. (2005) Hydrothermal convection in carbonaceous chondrite parent bodies. Earth Planet. Sci. Lett. 240, 234-250.

563. Trieloff M., Jessberger E. K., Herrwerth I., Hopp J., Fi_eni C., Gh_elis M., BourotDenise M., and Pellas P. (2003) Structure and thermal history of the H-chondrite parent asteroid revealed by thermochronometry. Nature 422, 502- 506.

564. Trigo-Rodríguez J. M., Rubin A. E. and Wasson J. T. (2006) Non-nebular origin of dark mantles around chondrules and inclusions in $\mathrm{CM}$ chondrites. Geochimica et Cosmochimica Acta 70, 1271-1290.

565. Trinquier A. Birck J.-L., and Allegre C. J. (2007) Widespread 54Cr heterogeneity in the inner solar system. The Astrophysical Journal 655, 1179-1185.

566. Tsuchiyama, A., Mashio, E., Imai, Y., Noguchi, T., Miura, Y., Yano, H., and Nakamura, T. (2009). Strength measurement of carbonaceous chondrites and micrometeorites using micro compression testing machine (abstract). Meteoritics \& Planetary Science Supplement., p.5189.

567. Tsuchiyama, A., Uesugi, M., Matsushima, T., Michikami, T., Kadono, T., Nakamura, T., Uesugi, K., Nakano, T., Sandford, S.A., Noguchi, R., Matsumoto, T., Matsuno, J., Nagano, T., Imai, Y., Takeuchi, A., Suzuki, Y., Ogami, T., Katagiri, J., 
Ebihara, M., Ireland, T.R., Kitajima, F., Nagao, K., Naraoka, H., Noguchi, T.,Okazaki, R., Yurimoto, H., Zolensky, M.E., Mukai, T., Abe, M., Yada, T., Fujimura, A., Yoshikawa, M., and Kawaguchi, J., (2011) Three-dimensional structure of Hayabusa samples: Origin and evolution of Itokawa regolith. Science 333,1125-1128.

568. Tsuchiyama A., Uesugi M., Uesugi K., et al., (2014) Three-dimensional microstructure of samples recovered from asteroid 25143 Itokawa: Comparison with LL5 and LL6 chondrite particles. Meteoritics \& Planetary Science 49, 172-187.

569. Tyra M. A. (2013). Using oxygen and carbon stable isotopes, ${ }^{53} \mathrm{Mn}-{ }^{53} \mathrm{Cr}$ isotope systematics, and petrology to constrain the history of carbonates and water in the CR and CM chondrite parent bodies. ProQuest Dissertations And Theses; Thesis (Ph.D.)--The University of New Mexico, 2013.; Publication Number: AAT 3588093; ISBN: 9781303260063; Source: Dissertation Abstracts International, Volume: 74-11(E), Section: B.; 235 p.

570. Ushikubo T., Kimura M., Kita N. T. and Valley J. W. (2012). Primordial oxygen isotope reservoirs of the solar nebula recorded in chondrules in Acfer 094 carbonaceous chondrite. G Geochimica et Cosmochimica Acta 90, 242-264.

571. Van Schmus W. R. and Hayes J. M. 1974. Chemical and petrographic correlations among carbonaceous chondrites. Geochimica et Cosmochimica Acta 38, 47-64.

572. Van Schmus W. R. and Wood J. A. (1967). A chemical petrologic classification for the chondritic meteorites. Geochimica et Cosmochimica Acta 31, 747-765.

573. Velbel M. A. (2014) Terrestrial weathering of ordinary chondrites in nature and continuing during laboratory storage and processing: Review and implications for Hayabusa sample integrity. Meteoritics \& Planetary Science 49, 154-171.

574. Velbel M. A. and Harvey R. P. (2009) Along-track compositional and textural variation in extensively melted grains returned from Comet 81P/Wild 2 by the Stardust mission: Implications for capture-melting process. Meteoritics \& Planetary Science. 44, 1519-1540.

575. Velbel M. A. and Palmer E. E. (2011) Fine-grained serpentine in CM2 carbonaceous chondrites and its implications for the extent of aqueous alteration on the parent body: A review. Clays Clay Min. 59, 416-432.

576. Velbel M. A., Tonui E. K. and Zolensky M. E. (2012) Replacement of olivine by serpentine in the carbonaceous chondrite Nogoya (CM2). Geochimica et Cosmochimica Acta 87, 117-135.

577. Velbel, M.A., Tonui, E.K., and Zolensky, M.E. (2015) Replacement of olivine by serpentine in the Queen Alexandra Range 93005 carbonaceous chondrite (CM2): Reactant-product compositional relations, and isovolumetric constraints on reaction stoichiometry and elemental mobility during aqueous alteration. Geochimica et Cosmochimica Acta 148, 402-425.

578. Vernazza P., Zanda B., Binzel R. P., Hiroi T., DeMeo F. E., Birlan M.; Hewins R., Ricci L., Barge P., and Lockhart M. (2014) Multiple and fast: The accretion of ordinary chondrite parent bodies. Astrophys. J., 791, Article ID 120.

579. Vernazza P., Zanda B., Nakamura T., Scott E. R. D., and Russell S. (2015) The Formation and Evolution of Ordinary Chondrite Parent Bodies. In Asteroids IV (P. Michel et al., eds.), 617-634. Univ. of Arizona, Tucson.

580. Veverka J., Robinson M., Thomas P., Murchie S., Bell J. F., Izenberg N., Chapman C., Harch A., Bell M., Carcich B., Cheng A., Clark B., Domingue D., Dunham D., Farquhar R., Gaffey M. J., Hawkins E., Joseph J., Kirk R., Li H., Lucey P., Malin M., Martin P., McFadden L., Merline W. J., Miller J. K., Owen W. M., Peterson C., Prockter L., Warren 
J., Wellnitz D., Williams B. G., and Yeomans D. K. (2000) NEAR at Eros: Imaging and spectral results. Science, 289 (5487), 2088-2097.

581. Veverka J., Thomas P. C., Robinson M., Murchie S., Chapman C., Bell M., Harch A., Merline W. J., Bell J. F., Bussey B., Carcich B., Cheng A., Clark B., Domingue D., Dunham D., Farquhar R., Gaffey M. J., Hawkins E., Izenberg N., Joseph J., Kirk R., Li H., Lucey P., Malin M., McFadden L., Miller J. K., Owen W. M., Peterson C., Prockter L., Warren J., Wellnitz D., Williams B. G., and Yeomans, D. K. (2001a) Imaging of small scale features on 433 Eros from NEAR: Evidence for a complex regolith. Science 292, 484-488.

582. Veverka J., Farquhar B., Robinson M., Thomas P., Murchie S., Harch A., Antreasian P. G., Chesley S. R., Miller J. K., Owen W. M., Williams B. G., Yeomans D., Dunham D., Heyler G., Holdridge M., Nelson R. L., Whittenburg K. E., Ray J. C., Carcich B., Cheng A., Chapman C., Bell J. F., Bell M., Bussey B., Clark B., Domingue D., Gaffey M. J., Hawkins E., Izenberg N., Joseph J., Kirk R., Lucey P., Malin M., McFadden L., Merline W. J., Peterson C., Prockter L., Warren J., and Wellnitz D. (2001b) The landing of the NEAR-Shoemaker spacecraft on asteroid 433 Eros. Nature 413, 390-393.

583. Vilas (2008) Spectral Characteristics of Hayabusa 2 Near-Earth Asteroid Targets 1621731999 JU3 and 2001 QC34. Astrophisical Journal 135, 1101-1105.

584. Villeneuve J., Libourel G., Soulié C. (2015) Relationships between type I and type II chondrules: Implications on chondrule formation processes. Geochim. Cosmochim Acta, 160, 277-305.

585. Vokrouhlický D., Broź M., Morbidelli A., Bottke W. F., Nesvorný D., Lazzaro D. and Rivkin A. S. (2006) Yarkovsky footprints in the Eos family. Icarus 182, 92-117.

586. Wark D. A. (1979) Birth of the presolar nebula: The sequence of condensation revealed in the Allende meteorite. Astrophys. Space Sci. 60, 59-75.

587. Wark D. A. (1981) Alteration and metasomatism of Allende Ca-Al-rich materials. 12th Lunar and Planetary Science Conference. pp. 1145-1147.

588. Wark D. A. (1987) Plagioclase-rich inclusions in carbonaceous chondrite meteorites: Liquid condensates? Geochimica et Cosmochimica Acta 51, 221-242.

589. Wark D. and Boynton W.V. (2001) The formation of rims on calcium-aluminum-rich inclusions: Step I. (2001) The forMeteoritics \& Planetary Science 36, 1135-1166.

590. Wark D., Boynton W.V., Keays R. and Palme H. (1987) Trace element and petrologic clues to the formation of forsterite-bearing $\mathrm{Ca}$-Al-rich inclusions in the Allende meteorite. Geochimica et Cosmochimica Acta 51, 607-622.

591. Wark D.A. and Lovering J.F. (1977) Marker events in the early evolution of the solar system: Evidence from rims on $\mathrm{Ca}-\mathrm{Al}$-rich inclusions in carbonaceous chondrites. Proceedings Lunar Science Conference 8, 95-112.

592. Warren P. H. (2011a) Stable-isotope anomalies and the accretionary assemblage of the Earth and Mars: A subordinate role for carbonaceous chondrites. Earth Planet. Sci. Lett. 331, 93-100.

593. Warren P. H. (2011b) Stable isotopes and the noncarbonaceous derivation of ureilites, in common with nearly all differentiated planetary materials. Geochimica et Cosmochimica Acta 75, 6912-6926.

594. Wasserburg G. J., Lee T. and Papanastassiou D. A. (1977) Correlated O and Mg isotopic anomalies in Allende inclusions: II. Magneisum. Geophys. Res. Lett. 4, 299-302.

595. Wasson J. T. (2008) Evaporation of nebular fines during chondrule formation. Icarus 195, 895-907.

596. Wasson J. T., Isa J., and Rubin A. E. (2013) Compositional and petrographic similarities of $\mathrm{CV}$ and $\mathrm{CK}$ chondrites: A single group with variations in textures and volatile 
concentrations attributable to impact heating, crushing, and oxidation. Geochimica et Cosmochimica Acta 108, 45-62.

597. Wasson J., Kallemeyn G. (1988) Compositions of chondrites. Phil. Trans R. Soc. Lond. A325, 535-544.

598. Wasson J., Kallemeyn G. (1990) Allan Hills 85085: A subchondritic meteorite of mixed nebular and regolithic heritage. Earth Planet. Sci. Lett, 101, 148-161.

599. Wasson J. T. and Rubin A. E. (2009) Composition of matrix in the CR chondrite LAP 02342. Geochimica et Cosmochimica Acta 73, 1436-1460.

600. Wasson J. T. and Rubin A. E. (2010) Matrix and whole-rock fractionations in the Acfer 094 type 3.0 ungrouped carbonaceous chondrites. Meteoritics \& Planetary Science. 45, 73-90.

601. Wasson J. T. and Rubin A. E. (2014) Matrix and whole-rock fractionations in the Acfer 094 Absence of matrix-like chondrule rims in CR2 LAP 02342. Meteoritics \& Planetary Science, 49, 245-260.

602. Wasson J. T. and Trigo-Rodríguez J. M. (2004) Evaporation during chondrule formation, recondensation as fine particles, and the condensation of $S$ and other volatile elements. Lunar Planet. Sci. 35 (abstract\#2140).

603. Wasson J. T., Yurimoto H., and Russell S. S. (2001). ${ }^{16} \mathrm{O}$-rich melilite in CO3.0 chondrites. Possible formation of common, ${ }^{16} \mathrm{O}$-poor melilite by aqueous alteration. Geochimica et Cosmochimica Acta, 65, 4539-4549.

604. Weidenschilling S. J. (2003) Radial drift of particles in the solar nebula: Implications for planetesimal formation. Icarus 165, 438-442.

605. Weisberg M. K. and Huber H. (2007) The GRO 95577 CR1 chondrite and hydration of the CR parent body. Meteoritics and Planetary Science 42, 1495-1503.

606. Weisberg M., Kimura M. (2012) The unequilibrated enstatite chondrites. Chemie de Erde 72, 101-115.

607. Weisberg M. K., McCoy T. J. and Krot A. N. (2006) Systematics and evaluation of meteorite classification. In Meteorites and the Early Solar System II (eds. D. Lauretta, L. A. Leshin and H. Y. McSween Jr.). University of Arizona Press, Tucson, pp. 19-52.

608. Weisberg M. K., Prinz M., Clayton R. N. and Mayeda T. K. (1997) CV3 chondrites: three subgroups, not two (abstract). Meteoritics \& Planetary Science 32, A138-A139.

609. Weisberg M. K., Prinz M., Clayton R., and Mayeda T. (1993) The CR (Renazzotype) carbonaceous chondrite group and its implications. Geochimica et Cosmochimica Acta 57, 1567-1586.

610. Weisberg M. K., Prinz M., Clayton R. N., Mayeda T. K., Grady M. M., and Pillinger C. T. (1995). The CR chondrite clan. Antarctic Meteorite Research 8, 11.

611. Weisberg M. K., Prinz M., Clayton R. N., Mayeda T. K., Sugiura N., Zashu S., and Ebihara M. 2001. A new metal-rich chondrite grouplet. Meteoritics \& Planetary Science 36, 401-418.

612. Weisberg M. K., Zolensky M. E., Kimura M., Ebel D. S., (2014) Primitive finegrained matrix in the unequilibrated enstatite chondrites. $45^{\text {th }}$ Lunar and Planetary Science Conference, Abstract \#1551.

613. Weiss B. P., Gattacceca J., Stanley S., Rochette P. and Christensen U. R. (2010) Paleomagnetic records of meteorites and early planetary differentiation. Space Sci. Rev. 152, 341-390.

614. Wick M. (2010). Formation conditions of plagioclase-bearing type I chondrules in CO chondrites: a study of natural samples and experimental analogs. M.S. thesis, University of New Mexico. 
615. Williams, I.P. and Wu, Z. (1993) The Geminid meteor stream and asteroid 3200 Phaethon. Mon. Not. R. Astron. Soc. 262, 231-248.

616. Wood J. A. (2003). Planetary science: Of asteroids and onions. Nature 422, 479481.

617. Wood J. A. (2004) Formation of chondritic refractory inclusions: The astrophysical setting. Geochimica et Cosmochimica Acta 68, 4007-4021.

618. Wulf A. Y., Palme H. and Jochum K. P. (1995) Fractionation of volatile elements in the early solar system: Evidence from heating experiments on primitive meteorites. Planet. Space Sci. 43, 451-468.

619. Yamamoto, D., Tachibana, S., Nagahara, H., Ozawa, K., and Tsuchiyama, T. (2015) Experimental study on hydrous mineral formation reaction between amorphous forsterite and water vapor. Lunar and Planetary Science XLVI, Abstract \#1930.

620. Yano H., Kubota T., Miyamoto H., et al. (2006) Touchdown of the Hayabusa spacecraft at the Muses Sea on Itokawa. Science 312(5778), 1350-1353.

621. Yin Q.-Z., Sanborn M. E., and Ziegler K. (2017) Testing the common source hypothesis for $\mathrm{CV}$ and $\mathrm{CK}$ chondrites parent body using $\triangle 17 \mathrm{O}-\varepsilon 54 \mathrm{Cr}$ isotope systematics. 48th Lunar and Planetary Science Conference, \#1771 (abstract).

622. Yingst, R. A., Haldemann, A. F. C., Biedermann, K. L., and Monhead, A. M. (2007). Quantitative morphology of rocks at the Mars Pathfinder landing site. Journal of Geophysical Research 112, E06002, doi:10.1029/2005JE002582

623. Yingst, R. A., Crumpler, L., Farrand, W. H., Li, R., Cabrol, N. A., and Neakrase, L. D. (2008). Morphology and texture of particles along the Spirit rover traverse from sol 450 to sol 745. Journal of Geophysical Research 113, E12S41, doi:10.1029/2088JE003179

624. Yoder H. S. Jr. (1973) Melilite stability and paragenesis. Fortschritte der Mineralogie 50, 140-173.

625. Yoshikawa M., Kawaguchi J., Fujiwara A., et al., (2015), Hayabusa Sample Return Mission. in Michel P., DeMeo F. E., Bottke W. F., eds, Asteroids IV . Univ. Arizona Press, Tucson, p. 767.

626. Young E. D. (2001) The hydrology of carbonaceous chondrite parent bodies and the evolution of planet progenitors. Phil. Trans. Roy. Soc. London A 359, 2095-2110.

627. Young E. D., Ash R. D., England P. and Rumble D. R. (1999) Fluid flow in chondritic parent bodies: Deciphering the compositions of planetsimals. Science 286, 1331-1335.

628. Young E. D., Zhang K. K. and Schubert G. (2003) Conditions for pore water convection within carbonaceous chondrite parent bodies - implications for planetesimal size and heat production. Earth Planet. Sci. Letters 213, 249-259.

629. Yurimoto H., Abe K.-I., Abe M., Ebihara M., Fujimora A., Hashiguchi M., Hashizume K., Ireland T. R., Itoh S., Katamaya J., Kato C., Kawaguchi J., Kawasaki N., Kitajima F., Kobayashi J., Meike T., Mukai T., Nagao K., Nakamura T., Naraoka H., Noguchi Ta., Okazaki R., Park C., Sakamoto N., Seto Y., Takei M., Tsuchiyama A., Uesugi M., Wakaki S., Yada T., Yamamoto K., Yoshikawa M., and Zolensky M. E., 2011. Oxygen isotopic compositions of asteroidal materials returned from Itokawa by the Hayabusa mission. Science 333, 1116-1119.

630. Zhao X., Floss C., Lin Y., and Bose M. (2013). Stardust Investigation into the CR Chondrite Grove Mountain 021710. The Astrophysical Journal, 769, article id. 49, 16 pp.

631. Zipfel J., Palme H., Clayton R. N., Mayeda T. K., Spettel B. and Wolf D. (2000) Dar al Gani 431: a new anomalous CK3 chondrite? 31th Lunar and Planetary Science Conference, \#1668 (abstract). 
632. Zolensky M. (1987) Tochilinite in C2 carbonaceous chondrites: A review with suggestions. Lunar Planet. Sci. XVIII, 1132-1133.

633. Zolensky M. (2002) Searching for samples of asteroid ponded deposits in meteorites. Antarctic Meteorites XXVII: Twentysixth Symposium on Antarctic Meteorites.

634. Zolensky M.E., Hewins R.H., Mittlefehldt D.W., Lindstrom M.M., Lipschutz M.E., Xiao X. (1993) Mineralogy, petrology and geochemistry of carbonaceous chondritic clasts in the LEW 85300 ploymict eucrite. Meteoritics \& Planetary Science 27, 596-604.

635. Zolensky M.E. (1995) Cyclical regolith processes on hydrous asteroids (abstract). Meteoritics 30, 606-607.

636. Zolensky M. E., Barrett R. and Browning L. (1993) Mineralogy and composition of matrix and chondrule rims in carbonaceous chondrites. Geochimica et Cosmochimica Acta 57, 3123-3148.

637. Zolensky M., Gounelle M., Mikouchi T., Ohsumi K., Le L., Hagiya K., Tachikawa O. (2008) Andreyivanovite: A second new phosphide from the Kaidun meteorite. American Mineralogist 93, 1295-1299.

638. Zolensky, M., Gregory, T., Takenouchi, A., Nishiizumi, K., Treiman, A., Berger, E., Le, L., Fagan, A., Velbel, M., Imae, N., and Yamaguchi, A. (2015). CM Carbonaceous Chondrite Lithologies and Their Space Exposure Ages. NIPR Annual Conference on Antarctic Meteorites, Abstracts, p. 226-227.

639. Zolensky M. and Ivanov A. (2003) The Kaidun microbreccia meteorite: A harvest from the inner and outer asteroid belt. Chem. der Erde 63, 185-246.

640. Zolensky M., Mackinnon I. D. R. (1986) Microstructures of cylindrical tochilinites. Amer. Mineral. 71, 120 l-1209.

641. Zolensky M. and McSween H. Y. Jr. (1988). Aqueous alteration. In Meteorites and the early solar system, edited by Kerridge J. F. and Shapley Matthews M. Tucson: The University of Arizona Press, Arizona, USA. pp. 114-143.

642. Zolensky M., Mikouchi T., Fries M., Bodnar R., Jenniskens P., Yin Q-.Z., Hagiya K., Ohsumi K., Komatsu M., Colbert M., Hanna R., Maisano J., Ketcham R., Nakamura T., Matsuoka M., Sasaki S., Tsuchiyama A., Gounelle G., Le L., Martinez J., Ross K., Rahman Z. (2014) Mineralogy and petrography of C asteroid regolith: The Sutter's Mill meteorite. Meteoritics \& Planetary Science.. 49, 1997-2016.

643. Zolensky M., Mikouchi T., Hagiya K., Ohsumi K., Komatsu M., Chan Q., Le L., Kring D., Cato M., Fagan A., Gross J., Tanaka A., Takegawa D., Hoshikawa T., Yoshida T., Sawa N. (2016) Unique view of C asteroid regolith from the Jbilet Winselwan CM chondrite. 47th Lunar and Planetary Science Conference. Abstract.

644. Zolensky M., Mikouchi T., Hagiya K., Ohsumi K., Komatsu M., Le L. (2015) Evidence for impact shock melting in $\mathrm{CM}$ and $\mathrm{Cl}$ chondrite regolith samples. 46th Lunar and Planetary Science Conference. Abstract.

645. Zolensky M. E., Mittlefehldt D. W., Lipschutz M. E., Wang M.-S., Clayton R. N., Mayeda T. K., Grady M. M., Pillinger C. and Barber D. (1997) CM chondrites exhibit the complete petrologic range from type 2 to 1 . Geochimica et Cosmochimica Acta 61, 50995115.

646. Zolensky M. E., Nakamura K., Gounelle M., Mikouchi T., Kasama T., Tachikawa O., Tonui E. (2002) Mineralogy of Tagish Lake: An ungrouped type 2 carbonaceous chondrite. Meteoritics \& Planetary Science 37, 737-761.

647. Zolensky M. E., Nakamura-Messenger K., Rietmeijer F., Leroux H., Mikouchi T., Ohsumi K., Simon S., Grossman L., Stephan T., Weisberg M., Velbel M., Zega T., Stroud R., Tomeoka K., Ohnishi I., Tomioka N., Nakamura T., Matrajt G., Joswiak D., Brownlee 
D., Langenhorst F., Krot A., Kearsley A., Ishii H., Graham G., Dai Z. R., Chi M., Bradley J., Hagiya K., Gounelle M. and Bridges J. (2008) Comparison of Wild-2 particles to chondrites and IDPs. Meteoritics \& Planetary Science 43, 261-272.

648. Zolensky M. E., Takenouchi A., Gregory T., Nishiizumi K., Caffee M., Velbel M., Ross K., Zolensky A., Le L., Imae N., Yamaguchi A., Mikouchi T. (2017) The relationship between cosmic-ray exposure ages and mixing of $\mathrm{CM}$ chondrite lithologies. 48th Lunar and Planetary Science Conference. Abstract.

649. Zolensky M. E., Weisberg M. K., Buchanan P. C., Prinz M., Reid A., and Barrett R. A. (1992). Mineralogy of dark clasts in CR chondrites, eucrites and howaardites (abstract). Lunar Planet. Sci. 23, 1587-1588.

650. Zolensky M. E., Weisberg M. K., Buchanan P. C. and Mittlefehldt D. W. (1996) Mineralogy of carbonaceous chondrite clasts in howardites, eucrites and the Moon. Meteoritics \& Planetary Science 31, 518-537.

651. Zolensky M. E., Zega T. J., Yano H., Wirick S., Westphal A. J., Weisberg M. K., Weber I., Warren J. L., Velbel M. A., Tsuchiyama A., Tsou P., Toppani A., Tomioka N., Tomeoka K., Teslich N., Taheri M., Susini J., Stroud R., Stephan T., Stadermann F. J., Snead C. J., Simon S. B., Simionovici A., See T. H., Robert F., Rietmeijer F. J. M., Rao W., Perronnet M. C., Papanastassiou D. A., Okudaira K., Ohsumi K., Ohnishi I., Nakamura-Messenger K., Nakamura T., Mostefaui S., Mikouchi T., Meibom A., Matrajt G., Marcus M. A., Leroux H., Lemelle L., Le L., Lanzirotti A., Langenhorst F., Krot A., Keller L. P., Kearsley A., Joswiak D., Jacob D., Ishii H., Harvey R., Hagiya K., Grossman L., Grossman J. N., Graham G. A., Gounelle M., Gillet P., Genge M. J., Flynn G. J., Ferroir T., Fallon S., Ebel D. S., Dai Z. R., Cordier P., Chi M., Butterworth A. L., Brownlee D. E., Browning N., Bridges J. C., Brennan S., Brearley A., Bradley J. P., Bland P., and Bastien R. (2006) Mineralogy and Petrology of Comet 81P/Wild 2 Nucleus Samples. Science 314, 1735-1739.

652. Zolensky M., Ziegler K., Weisberg M., Gounelle M., Berger E., Le L., Ivanov A. (2014) Aqueous alteration of enstatite chondrites. $45^{\text {th }}$ Lunar and Planetary Science Conference, Abstract.

653. Zolotov M. Y. (2012). Aqueous fluid composition in Cl chondritic materials: Chemical equilibrium assessments in closed systems. Icarus 220, 713-729.

Tables:

Table 2.1. C-chondrite groups: Relative numerical abundances and proportions

\begin{tabular}{|c|c|c|c|c|c|c|c|}
\hline $\begin{array}{c}\text { C-chondrite } \\
\text { class }\end{array}$ & $\begin{array}{c}\text { Ave. } \rho \\
\left(\mathrm{g} / \mathrm{cm}^{3}\right)\end{array}$ & $\begin{array}{c}\text { Mean } \\
\text { wt. \% } \\
\mathrm{H}_{2} \mathrm{O}^{1}\end{array}$ & $\begin{array}{c}\text { Mean } \\
\text { wt.\% } \\
\mathrm{C}^{1}\end{array}$ & $\begin{array}{c}\text { number of } \\
\text { cataloged } \\
\text { meteorites }^{1}\end{array}$ & $\%^{1}$ & $\begin{array}{c}\text { number of } \\
\text { cataloged } \\
\text { meteorites }^{2}\end{array}$ & $\%^{2}$ \\
\hline $\mathrm{Cl}$ & 1.58 & 18.0 & 3.2 & 5 & 3 & 5 & 1 \\
\hline $\mathrm{CM}$ & 2.20 & 12.6 & 2.2 & 49 & 28 & 161 & 29 \\
\hline $\mathrm{CR}$ & 3.11 & 5.7 & 1.44 & 18 & 10 & 78 & 14 \\
\hline
\end{tabular}




\begin{tabular}{|c|c|c|c|c|c|c|c|}
\hline CV & 3.03 & 2.5 & 0.56 & 28 & 16 & 49 & 9 \\
\hline $\mathrm{CK}$ & 2.90 & 0.8 & $\sim 0.1$ & 28 & 16 & 73 & 13 \\
\hline $\mathrm{CO}$ & 3.03 & 0.6 & 0.45 & 37 & 21 & 78 & 14 \\
\hline $\mathrm{CH}$ & & 0.1 & $\sim 0.8$ & 11 & 6 & 11 & 2 \\
\hline $\mathrm{CB}$ & 5.25 & & & & & & \\
\hline Unclassified & & & & & & 91 & 16 \\
\hline Ungrouped & & & & & & 8 & 1 \\
\hline Total CCs & & & & 176 & 100 & 554 & 100 \\
\hline OC & & trace & $\sim 0.1$ & & & & \\
\hline
\end{tabular}

${ }^{1}$ Hutchison (2004); ${ }^{2}$ Grady (2000)

Table 2.4. CM2 aqueous-alteration scales and measurable alteration parameters.

\begin{tabular}{|c|c|c|c|c|c|}
\hline Meteorite & Stage $^{1}$ & $\begin{array}{l}\text { Petrologic CM } \\
\text { sub-type }^{2}\end{array}$ & $\begin{array}{c}\text { Bulk } H \\
\text { atoms } H / 100 \text { atoms } \mathrm{Si}^{3}\end{array}$ & $\mathrm{MAI}^{4}$ & $\begin{array}{l}\text { Phyllosilicate/ } \\
\text { Anhydrous }^{5}\end{array}$ \\
\hline Paris & 0 & $2.7-2.9$ & & & \\
\hline QUE 97990 & & 2.6 & & & 2.19 \\
\hline Y 791198 & & 2.4 & & & 2.64 \\
\hline Bells & & & 178 & 0.46 & 4.03 \\
\hline Murchison & 1 & 2.5 & 214 & 0.43 & 3.10 \\
\hline Pollen & & & 236 & 0.53 & \\
\hline Erakot & & & 281 & & \\
\hline Boriskino & & & 290 & 0.73 & \\
\hline Murray & 1 & $2.4 / 5$ & 292 & 0.57 & 3.24 \\
\hline LEW 90500 & 3 & & & & 3.45 \\
\hline Mighei & 2 & & 313 & 0.77 & 3.73 \\
\hline ALH 81002 & 4 & & & & 3.96 \\
\hline Haripura & & & 346 & & \\
\hline Nogoya & 5 & 2.2 & 351 & 0.97 & 4.16 \\
\hline MAC 88101 & & & & & 4.16 \\
\hline Essebi & & & 279 & & 4.49 \\
\hline Cold Bokkeveld & & 2.2 & 378 & 1.03 & 4.72 \\
\hline QUE 93005 & 6 & 2.1 & & & 6.57 \\
\hline MET 01070 & & 2.0 & & & 11.36 \\
\hline
\end{tabular}

Meteoites are only included in this Table if the same meteorite has been assigned a value for at least two of the alteration parameters shown, in published peer-reviewed papers. These are selected to illustrate the extent to which the different alteration parameters do or do not show similar trends for thte same meteorites. Many more meteorites have been classified by only one of these alteration parameters, or by others. References: ${ }^{1}$ Hanowski and Brearley (2001), extended by Velbel et al. (2012, 2015); ${ }^{2}$ Rubin et al. (2007), extended by Velbel et al. (2012, 2015), Hewins et al. (2014), 
and Marrocchi et al. (2014); ${ }^{3}$ Van Schmus and Hayes (1974) and Kerridge (1985); ${ }^{4}$ Browning et al., (1996); ${ }^{5}$ Howard et al. $(2011,2015)$.

Table 2.2. Common minerals in chondrites

\begin{tabular}{|c|c|}
\hline Mineral name & Mineral formula \\
\hline Olivine (var. forsterite, fayalite $)$ & $(\mathrm{Mg}, \mathrm{Fe})_{2} \mathrm{SiO}_{4}$ \\
\hline Pyroxene (many var.) $(x=$ mol fraction $\mathrm{Wo})$ & $\mathrm{Ca}_{x}(\mathrm{Mg}, \mathrm{Fe})_{2-x} \mathrm{Si}_{2} \mathrm{O}_{6}$ \\
\hline Plagioclase feldspar $(x=$ mol fraction $\mathrm{An})$ & $\mathrm{Na}(1-x) \mathrm{Ca}_{x} \mathrm{Al}_{(1+x) \mathrm{Si}_{(3-x)} \mathrm{O}_{8}}$ \\
\hline Sulfides & $\mathrm{FeS}$ \\
\hline Metal & $\mathrm{Fe}, \mathrm{Ni}$ \\
\hline Oxides (e.g., magnetite) & $\mathrm{Fe}(\mathrm{III})_{2} \mathrm{Fe}(\mathrm{II}) \mathrm{O}_{4}$ \\
\hline Serpentine & $(\mathrm{Mg}, \mathrm{Fe})_{3} \mathrm{Si}_{2} \mathrm{O}_{5}(\mathrm{OH})_{4}$ \\
\hline Saponite & $\mathrm{Ca}_{0.25}(\mathrm{Mg}, \mathrm{Fe})_{3}\left((\mathrm{Si}, \mathrm{Al})_{4} \mathrm{O}_{10}\right)(\mathrm{OH})$ \\
\hline Glass (mesostasis) and alteration products & \\
\hline
\end{tabular}

Table 3. Serpentine-group minerals and their formulae.

\begin{tabular}{|c|c|c|}
\hline Group & Name & Formula \\
\hline \multirow{4}{*}{ Mg-bearing } & Chrysotile & $\mathrm{Mg}_{3} \mathrm{Si}_{2} \mathrm{O}_{5}(\mathrm{OH})_{4}$ \\
\cline { 2 - 3 } & Lizardite & $\mathrm{Mg}_{3} \mathrm{Si}_{2} \mathrm{O}_{5}(\mathrm{OH})_{4}$ \\
\cline { 2 - 3 } & Antigorite & $\mathrm{Mg}_{3} \mathrm{Si}_{2} \mathrm{O}_{5}(\mathrm{OH})_{4}$ \\
\hline \multirow{4}{*}{ Fe-bearing } & Cronstedtite & $\mathrm{Fe}(\mathrm{II})_{2} \mathrm{Fe}(\mathrm{III})\left(\mathrm{SiFe}^{3+}\right)_{\mathrm{O}_{5}(\mathrm{OH})_{4}}$ \\
\cline { 2 - 3 } & Greenalite & $(\mathrm{Fe}(\mathrm{II}), \mathrm{Fe}(\mathrm{III}))_{2-3} \mathrm{Si}_{2} \mathrm{O}_{5}(\mathrm{OH})_{4}$ \\
\cline { 2 - 3 } & Ferroan antigorite & $(\mathrm{Mg}, \mathrm{Fe}, \mathrm{Mn})_{3}(\mathrm{Si}, \mathrm{Al})_{2} \mathrm{O}_{5}(\mathrm{OH})_{4}$ \\
\hline \multirow{3}{*}{ Intermediate } & Amesite & $\mathrm{Mg} 2 \mathrm{Al}(\mathrm{SiAl}) \mathrm{O}_{5}(\mathrm{OH})_{4}$ \\
\cline { 2 - 3 } & Berthierine & $\left(\mathrm{Fe}(\mathrm{II}), \mathrm{Fe}(\mathrm{III})_{,}, \mathrm{Mg}\right)_{2-3}(\mathrm{Si}, \mathrm{Al})_{2} \mathrm{O}_{5}(\mathrm{OH})_{4}$ \\
\hline
\end{tabular}

Adapted from Zolensky and McSween (1988), Browning et al. (1996), and Velbel and Palmer (2011)

Table 2.6. Peak metamorphic temperatures of thermally altered $\mathrm{C}$ chondrites

\begin{tabular}{|l|c|c|}
\hline Meteorite & Classification* & $\mathbf{T}_{\text {peak metamorph. }}\left({ }^{\circ} \mathrm{C}\right)$ \\
\hline Y-86789 & CM2TIV & $>600^{* *} \leq 700^{*}=\downarrow$ \\
\hline Y-86720 & CM2TIV & $={ }^{*}$ \\
\hline Y-82162 & CI1TII/III & \\
\hline Y-86029 & CI1TIII & $=\downarrow^{*}$ \\
\hline B-7904 & CM2TIV & $={ }^{*}>600^{* *}$ \\
\hline
\end{tabular}


Zolensky et al.: Characteristics of Chondrites, 152

\begin{tabular}{|l|c|c|}
\hline PCA 91008 & CM2TIII & $*>600^{* *}$ \\
\hline A-881655 & CM2TIII & $=\downarrow^{*}$ \\
\hline EET 90043 & CM2TII/III & $=\uparrow^{*}$ \\
\hline WIS 91600 & CM2TII & $=\uparrow^{*}$ \\
\hline Y-793321 & CM2TII & $\geq 400^{*}$ \\
\hline
\end{tabular}

*From Tonui et al. (2014)

Arrows point to adjacent listed samples identified as having equal peak temperatures of metamorphism. 